\title{
Electronic Structure and Thermodynamic Properties of High Temperature Superconductors
}

\author{
By \\ James Glenn Storey
}

A thesis

submitted to Victoria University of Wellington in fulfilment of the requirements for the degree of

Doctor of Philosophy in Physics

Victoria University of Wellington 2007 


\section{Abstract}

The generic doping dependence of the thermodynamic, electrodynamic and transport properties of high-temperature superconductors remains a puzzle despite many years of study. We are still awaiting a rigorous scientific theory that explains the resistance-free flow of electric current in these novel materials. In conventional superconductors, observations of the predicted dependence of the superconducting transition temperature on isotopic mass played a key role in identifying a phononic pairing mechanism.

In order to elucidate the role of phonons in the high- $T_{c}$ superconductors, the oxygen isotope effect in the separate components of the penetration depth tensor of the high-temperature superconductor $\mathrm{YBa}_{2} \mathrm{Cu}_{4} \mathrm{O}_{8}$ was determined from AC susceptibility measurements, performed on biaxially-aligned powders set in epoxy. The results, extracted after assuming values for the upper cut-off radii in the particle size distributions, show that the isotope effect in the $b c$-plane is negligible compared to those of the $a b$ - and $a c$-planes. This suggests that the electrons prefer to couple to phonon modes in which the motion of the atoms is perpendicular to the plane of transport.

The electronic entropy, superfluid density, Raman response, spin susceptibility and thermoelectric power were calculated from energy-momentum dispersions determined by angle-resolved photoemission spectroscopy (ARPES). 
An excellent match with experimental data was obtained. This is a highly significant result because it provides the first comprehensive link between these bulk properties and the ARPES measurements which are dominated by the outermost $\mathrm{CuO}_{2}$ layer. Thus, in most respects surface effects do not appear to seriously modify or obscure the band structure which governs bulk properties. The calculations reveal the presence of a van Hove singularity (vHs) at the Fermi level $\left(E_{F}\right)$ in the heavily overdoped regime to be a universal feature of the cuprates. The evolution of these properties with temperature and doping can be fully explained by the retreat of $E_{F}$ from the vHs and the opening of a normal state pseudogap as doping is decreased. Consequently, the pairing potential amplitude is found to be a strongly decreasing function of hole concentration, similar to the doping dependence of the exchange interaction, $J$. The pairing interaction is possibly a universal function of the $E_{F}-E_{v H s}$ with the maximum in the transition temperature $\left(T_{c}\right)$ governed by the exact magnitude of the density of states on the flanks of the vHs. These are key new discoveries which may provide a route forward to solving the puzzle of high-temperature superconductivity. 


\section{Acknowledgements}

Firstly, it is a great pleasure to thank my principal supervisor Professor Jeffery Tallon of Victoria University of Wellington and Industrial Research Limited. No matter how hectic his schedule he was always willing to provide assistance. In addition to his vast knowledge and experience his consistently optimistic attitude was a key ingredient in making this project a success.

I am equally pleased to thank my second supervisor Dr Grant Williams of Industrial Research Ltd for many helpful discussions that have been instrumental to all aspects of this project. I would also like to thank him for kindly supplying the experimental data upon which the analysis in section 6.4 is based.

To my supervisors, it has been a great privilege to work with you both.

This project was carried out at Industrial Research Limited. I would like to thank the many staff members that have happily provided their assistance, especially Martin Ryan (X-Ray diffraction), Kay Card (scanning electron microscopy), Neville Baxter (particle size analysis), Mike Staines and Suresh Narayanaswamy. Thanks also to Associate Professor Pablo Etchegoin of Victoria University of Wellington (Raman spectroscopy).

Special thanks are due to the following people. Dr John Loram of the 
University of Cambridge, for his electronic entropy data which provided the anchor for the computational work performed in this project, and also for his helpful comments. Assistant Professor Adam Kaminski of Iowa State University and Dr Alexander Kordyuk of the Solid State Materials Institute in Dresden for kindly providing the tight binding parameters for energymomentum dispersions employed in this work.

This work was funded by the MacDiarmid Institute for Advanced Materials and Nanotechnology. Completion of this work was made possible thanks to a PhD Completion Scholarship from Victoria University of Wellington.

I am forever grateful to my parents Grant and Chikako, and my brother Adam for a lifetimes worth of love, support, education, guidance and encouragement. They are responsible for shaping me into the person that I am and without them I would be nothing. Thanks also to my grandfather Cecil who by teaching me the basics of electricity at a young age sparked my interest in science. Finally I would like to express my deepest thanks to my partner Julia, for her love, support and encouragement over the past six years, and for her unwavering belief in me even when I doubted myself. 


\section{Contents}

1 Introduction 1

2 Background $\quad 6$

2.1 Crystal Structure . . . . . . . . . . . . . . 6

2.2 Phase Diagram . . . . . . . . . . . . . . . . . . . . . 7

2.3 Angle Resolved Photoemission

Spectroscopy . . . . . . . . . . . . . . . . 12

2.4 Energy-momentum Dispersion . . . . . . . . . . . . . 14

2.4.1 Fermi Surface . . . . . . . . . . . . . . 16

2.4 .2 Bilayer Splitting . . . . . . . . . . . . . 18

2.4.3 Superconducting Gap . . . . . . . . . . . . . . . . 19

2.5 Van Hove Scenario . . . . . . . . . . . . . . . . . 22

2.6 Pseudogap . . . . . . . . . . . . . . . . . . . . . 23

2.7 Isotope Effect . . . . . . . . . . . . . . . . . . . . 25

2.8 Superfluid Density . . . . . . . . . . . . . . . . 29

3 Experimental Techniques 31

3.1 Preparation of $\mathrm{YBa}_{2} \mathrm{Cu}_{4} \mathrm{O}_{8} \ldots \ldots \ldots . \ldots . \ldots . \ldots$ 
3.2 Oxygen Isotope Exchange . . . . . . . . . . . . . . . . . 32

3.3 Biaxial Alignment . . . . . . . . . . . . . . . 34

3.4 Measurements . . . . . . . . . . . . . . . . 37

4 Experimental Results $\quad 39$

4.1 Sample characterization . . . . . . . . . . . . . . . 39

4.2 Magnetization ... . . . . . . . . . . . . 46

4.3 AC Susceptibility . . . . . . . . . . . . . . . . . 51

4.4 Penetration Depth \& Superfluid Density . . . . . . . . . . . 51

5 Computational Techniques $\quad 62$

5.1 Dispersion . . . . . . . . . . . . . . . 65

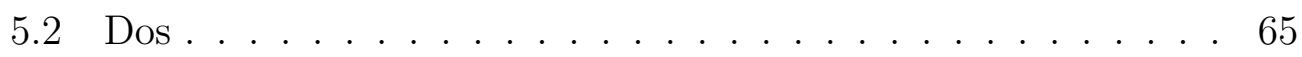

5.3 SCgap .......................... 66

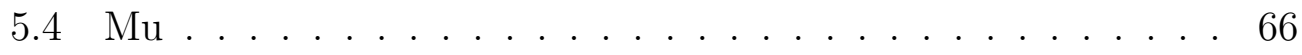

5.5 SFDensity . . . . . . . . . . . . . . . . . 68

5.6 VSum ........................... 70

5.7 Тер . . . . . . . . . . . . . . . . . 70

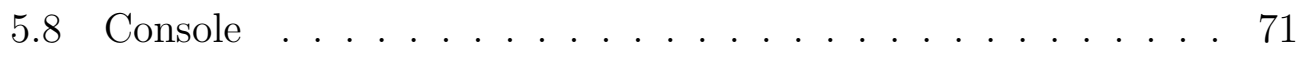

5.9 RunFindTc . . . . . . . . . . . . . . . . 72

5.10 FindTc . . . . . . . . . . . . . . . . . 74

6 Computational Results $\quad 75$

6.1 Electronic Entropy . . . . . . . . . . . . . . . . . 75

6.2 Superfluid Density . . . . . . . . . . . . . . . . 94

6.3 Raman Response . . . . . . . . . . . . . . . . . . . . . . . . 97 
6.4 Spin Susceptibility . . . . . . . . . . . . . . . 105

6.5 Thermoelectric Power . . . . . . . . . . . . . . . . . . . 109

6.6 Scattering Rate . . . . . . . . . . . . . . . . . . . . 122

6.7 Phase Diagram . . . . . . . . . . . . . . . . 130

6.8 Isotope Effect . . . . . . . . . . . . . . . . . . . . . 141

$\begin{array}{lll}7 & \text { Summary and Conclusions } & 145\end{array}$

7.1 Experimental Work . . . . . . . . . . . . . . 145

7.2 Computational Work . . . . . . . . . . . . . . 146

7.2 .1 Normal-state Findings . . . . . . . . . . . . . . 147

7.2.2 Superconducting-State Findings . . . . . . . . . . 149

Bibliography

155 


\section{List of Figures}

2.1 Crystal structure of Bi-2212. . . . . . . . . . . . . . 8

2.2 Crystal structure of $\mathrm{Y}-124 \ldots \ldots \ldots$

2.3 Antiferromagnetic structure of the copper oxide plane. . . . . 10

$2.4 \quad T-p$ phase diagram. . . . . . . . . . . . . . . 11

2.5 ARPES measurement geometry. . . . . . . . . . . . . . 14

2.6 Schematic diagram of electron orbitals in the $\mathrm{CuO}_{2}$ plane. . . 15

2.7 Energy momentum dispersion of Bi-2212. . . . . . . . . . 16

$2.8\left(k_{x}, k_{y}\right)$ projection of the Bi-2212 $\epsilon(\mathbf{k})$ dispersion. . . . . . . 17

2.9 The vHs boundary between Fermi surface topologies. . . . . . 18

2.10 Bi-2212 bilayer $\epsilon(\mathbf{k})$ dispersion. . . . . . . . . . . . . . 20

$2.11 d$-wave superconducting gap function. . . . . . . . . . . . 21

2.12 Two scenarios for $T^{*} \ldots \ldots \ldots \ldots$

3.1 Schematic diagram of the oxygen isotope exchange rig. . . . . 33

3.2 Schematic diagram of the biaxial alignment rig. . . . . . . . 36

4.1 XRD spectrum from a Y-124 sample pellet. . . . . . . . . . . 40

4.2 Raman spectra from ${ }^{16} \mathrm{O} /{ }^{18} \mathrm{O}$ substituted $\mathrm{Y}-124$ pellets. . . . . 41

4.3 XRD spectra from the biaxially aligned samples. . . . . . . . . 43 
4.4 Close up of the $[0,0,14],[0,2,0]$ and $[2,0,0]$ Bragg peaks. . . . . 44

4.5 Size distributions of ${ }^{16} \mathrm{O} /{ }^{18} \mathrm{O}$ substituted $\mathrm{Y}-124$ powders. . . . 47

4.6 SEM images of the grains in the composite samples. . . . . . . 48

4.7 Grain size distributions from SEM images. . . . . . . . . . . 49

4.8 DC magnetization of ${ }^{16} \mathrm{O} /{ }^{18} \mathrm{O}$ substituted Y-124 pellets. . . . 50

4.9 FC and ZFC magnetization of ${ }^{16} \mathrm{O} /{ }^{18} \mathrm{O}$ substituted $\mathrm{Y}-124$ pow-

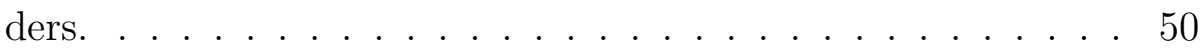

4.10 AC susceptibility of the biaxially aligned samples. . . . . . . . 52

4.11 Suppression of the normalised susceptibility as a function of $\lambda / r \ldots \ldots \ldots \ldots \ldots \ldots \ldots \ldots \ldots \ldots \ldots \ldots \ldots \ldots \ldots \ldots$

4.12 Variation of $\lambda_{a b}$ with upper cut-off radius. . . . . . . . . 55

4.13 Temperature dependence of $\rho_{a b}, \rho_{b c}$ and $\rho_{a c}$. . . . . . . . 58

4.14 Temperature dependence of $\rho_{a}, \rho_{b}$ and $\rho_{c} \ldots \ldots \ldots$

5.1 The Console program architecture. . . . . . . . . . . . 63

5.2 The method for calculating trial values of $\Delta_{0} \ldots \ldots$. . . . 67

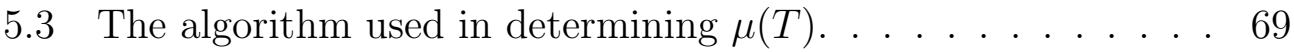

5.4 A screen capture of the Console program interface. . . . . . . 71

5.5 The RunFindTc program architecture. . . . . . . . . . . . 72

5.6 The algorithm used in determining $T_{c} \ldots \ldots . \ldots 73$

6.1 The antibonding band $\epsilon(\mathbf{k})$ dispersion with a $10 \mathrm{meV}$ pseudogap at $50 \mathrm{~K} \ldots \ldots \ldots \ldots \ldots \ldots \ldots$

6.2 Evolution of the model pseudogap with temperature. . . . . . 78

6.3 The density of states calculated from the Bi-2212 bilayer dis-

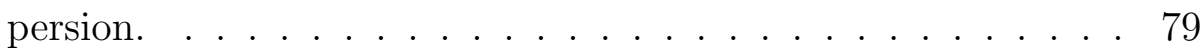


6.4 The specific heat coefficient for three pseudogap scenarios. . . 81

6.5 Simulated ARPES energy dispersion curves. . . . . . . . . . 82

6.6 Fits to the normal- and superconducting-state entropy. . . . . 85

6.7 Parameters determined from fits to the electronic entropy. . . 87

6.8 Fitted and measured $E_{F}-E_{v H s}$ values. . . . . . . . . 88

6.9 Electronic entropy vs temperature. . . . . . . . . . . . . . . 89

6.10 Electronic entropy vs doping at different temperatures. . . . . 90

6.11 Fits to overdoped LSCO entropy data using a Bi-2201 dispersion. 91

6.12 Pairing potential cut-off energy vs $E_{F}-E_{v H s} \ldots \ldots . \ldots 9$

6.13 Computed and measured superfluid density . . . . . . . . . 96

6.14 Calculated and measured Bi-2212 low- $T$ superfluid density. . . 97

$6.15 B_{1 g}$ and $B_{2 g}$ Raman vertex functions. . . . . . . . . . 99

6.16 Calculated Raman response for a non-nodal pseudogap. . . . . 100

6.17 Experimental Raman data for Hg-1201. . . . . . . . . . . . . . 101

6.18 Calculated Raman response for a fully nodal pseudogap. . . . 101

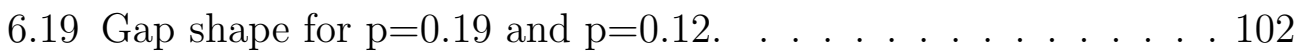

6.20 Fits to underdoped entropy data assuming a non-nodal pseudogap. ........................... 104

6.21 The density of states of Y-123. . . . . . . . . . . . 107

6.22 Fits to $\mathrm{Y}_{0.8} \mathrm{Ca}_{0.2} \mathrm{Ba}_{2} \mathrm{Cu}_{3} \mathrm{O}_{7-\delta}{ }^{89} \mathrm{Y}$ NMR Knight shift data. . . . 108

6.23 Measured and calculated thermopower of Bi-2201. . . . . . . . 111

6.24 The density of states and conductivity of Bi-2201. . . . . . . . 113

$6.25 T$-dependent scattering rate from IR reflectivity. . . . . . . . . 114

6.26 Bi-2201 thermopower fits. . . . . . . . . . . . . 116

6.27 Fits to underdoped Bi-2212 thermopower data. . . . . . . . . 118 
6.28 The scattering rates used in fitting the Bi-2212 thermopower. . 119

6.29 The bilayer Bi-2212 energy dependent conductivity. . . . . . . 120

6.30 Measured and calculated overdoped Bi-2212 thermopower. . . 121

6.31 Lifetime of the Bloch states at the Fermi level. . . . . . . . . . 123

6.32 Plot of the parameters used in fitting $\tau(\theta) \ldots . . . . . .125$

6.33 The conductivity calculated using an ARPES-derived relaxation time. . . . . . . . . . . . . . 126

6.34 The velocity field of Bi-2201. . . . . . . . . . . . . 127

6.35 The cosine of the angular deviation of the velocity from $45^{\circ} . \quad 128$

6.36 The cosine of the angular deviation of the velocity from $45^{\circ}$ as a function of Fermi surface angle. . . . . . . . . . . . . . 129

6.37 The conductivity calculated with $\tau$ given by $\cos \left[2\left(\phi-45^{\circ}\right)\right] . \quad .129$

6.38 The calculated $T-p$ phase diagram. . . . . . . . . . . . . 132

6.39 Energy resolved contributions to the BCS gap equation. . . . . 136

6.40 Calculated Bi-2201 $T_{c}(p)$ for different values of $t^{\prime} / t \ldots \ldots$. . . 138

$6.41 T_{c}$ 's for different cuprates assuming an identical $V\left(E_{F}-E_{v H s}\right) .140$

6.42 Doping dependence of the measured and calculated oxygen isotope effect coefficient. . . . . . . . . . . . . . . . . 142

6.43 Energy resolved contributions to the BCS gap equation at $p=0.136 \ldots \ldots \ldots \ldots \ldots \ldots \ldots \ldots \ldots \ldots$ 


\section{List of Tables}

4.1 Sample masses before and after oxygen isotope exchange. . . . 41

4.2 Oxygen isotope induced Raman shift. . . . . . . . . . . . . 42

4.3 Rocking curve full width half maximum values. . . . . . . . . 45

4.4 Parameters of the AC susceptibility samples. . . . . . . . . . 45

$4.5 a b$-plane penetration depths from SEM size distributions. . . . 54

$4.6 \lambda_{a b}, \lambda_{b c}$ and $\lambda_{a c}$ assuming fixed cut-off radii. . . . . . . . . 56

$4.7 \lambda_{a}, \lambda_{b}, \lambda_{c}$ and isotope effect coefficients. . . . . . . . 56

4.8 Plane and axis superfluid densities and isotope effect coefficients. 57

6.1 Bi-2212 bilayer dispersion tight binding basis functions and coefficients. . . . . . . . . . . . . 76

6.2 Tight binding basis functions and coefficients of Y-123. . . . 106

6.3 Values of Bi-2201 thermopower fit parameters. . . . . . . . . . 115 


\section{List of Publications}

Work carried out as part of this project contributed to the following publications.

1. J. L. Tallon, R. S. Islam, J. Storey, G. V. M. Williams and J. R. Cooper, Isotope effect in the superfluid density of high-temperature superconducting cuprates: stripes, pseudogap and impurities., Phys. Rev. Lett., 94, 237002 (2005).

2. T. R. Hammad, A. J. Zaleski and J. G. Storey, Probing the order parameter of magnetically grain-aligned NCCO electron-doped cuprate., Physica C, 449, 122 (2006).

3. J. G. Storey, J. L. Tallon and G. V. M. Williams, Thermodynamic properties of $\mathrm{Bi}_{2} \mathrm{Sr}_{2} \mathrm{CaCu}_{2} \mathrm{O}_{8}$ calculated from the electronic dispersion., arXiv:0704.2432 (2007).

4. J. G. Storey, J. L. Tallon and G. V. M. Williams, Tracking the pseudogap below $T_{c}$ by modeling the Raman response., Curr. Appl. Phys., accepted (2007). 
5. J. G. Storey, J. L. Tallon, G. V. M. Williams and J. W. Loram, Fermi arcs in cuprate superconductors: Tracking the pseudogap below $T_{c}$ and above $T^{*}$., Phys. Rev. B, 76, 060502(R) (2007).

6. J. G. Storey, J. L. Tallon and G. V. M. Williams, Saddle-point van Hove singularity and the phase diagram of high- $T_{c}$ cuprates., Phys. Rev. B, accepted (2007). 


\section{Chapter 1}

\section{Introduction}

Perhaps no other class of materials has been the target of such concentrated research as the high temperature superconducting cuprates. Twenty one years after the discovery of the first high temperature superconductor by Bednorz and Müller we are still awaiting a rigorous scientific theory that explains the origin of the resistance-free flow of electric current in these materials. If the history of superconductivity is anything to go by our patience will eventually be rewarded.

First discovered in 1911 by Kamerlingh Onnes[1, 2] it would be another 46 years until the phenomenon low-temperature or conventional superconductivity in metals would be explained by a theory proposed by Bardeen Cooper and Schrieffer[3]. BCS Theory, as it has become known, describes how the pairing of electrons can occur in the presence of an arbitrarily small attractive interaction and is now regarded a significant achievement of modern condensed matter theory. It has proceeded to have impacts on areas of physics well beyond the realm of the metallic systems it was initially in- 
tended to describe[4]. The diversity of its reach now extends from the pairing of neutrons and protons in nuclei[5], to the pairing of nucleons in neutron stars[6] and the behaviour of the exotic quark matter[7] speculated to exist inside their cores.

In the years between 1911 and 1974 the discovery of materials with higher superconducting transition temperatures $\left(T_{c}\right.$ 's) proceeded incrementally with an average rate of increase in $T_{c}$ of about three degrees per decade. Then for the next twelve years the highest $T_{c}$ achieved remained fixed at $23.3 \mathrm{~K}$. At the time it was widely believed that superconductivity above $30 \mathrm{~K}$ was impossible. It therefore came as a complete shock to the physics community when Bednorz and Müller announced the discovery of the onset of superconductivity in Ba-La-Cu-O at 35K[8, 9] (the superconducting phase was later identified to be $\left.\mathrm{La}_{2-x} \mathrm{Ba}_{x} \mathrm{CuO}_{4}[10]\right)$. The new 'high-temperature' superconductor belongs to a class of layered copper-oxide based ceramics or cuprates. Their relative ease of preparation spurred a worldwide race to find similar materials with yet higher $T_{c}$ 's. Barely a year later superconductivity above $90 \mathrm{~K}$ was discovered in $\mathrm{YBa}_{2} \mathrm{Cu}_{3} \mathrm{O}_{6+x}[11,12]$. To date the record at ambient pressure stands at $135 \mathrm{~K}$ in $\mathrm{HgBa}_{2} \mathrm{Ca}_{2} \mathrm{Cu}_{3} \mathrm{O}_{8+\delta}$ which can be increased to around $160 \mathrm{~K}$ via the application of pressure[13].

The quest for higher transition temperatures is driven by the obvious advantages that superconductors bring to technological applications. Until the arrival of high-temperature superconductivity (HTS) these advantages were largely outweighed by the costs associated with cooling the materials to the superconducting state. The lack of an understanding of the mechanism underlying high temperature superconductivity in the cuprates has 
not prevented their commercial exploitation. Such an understanding might however allow us to determine from first principles if higher transition temperatures are possible in this class of materials, or whether we have reached some fundamental limit and should direct our search elsewhere. To this end the cuprates have been subjected to the full arsenal of modern experimental probes.

Proposed mechanisms for electron pairing in high- $T_{c}$ superconductors can be described as either phonon based or non-phonon based (or maybe a combination of both). Phonons are quantized lattice vibrations. Observations of the predicted dependence of the superconducting transition temperature on isotopic mass[14] helped confirm the virtual exchange of phonons as the source of electron pairing in conventional low temperature superconductors. The role of phonons in HTS is not clear and so the aim of the experimental part of this project was to determine the isotope effect in the penetration depth tensor. The penetration depth, $\lambda$, is a fundamental length scale and is related to the superfluid density. The superfluid density, $\rho_{s} \propto \lambda^{-2}$, is a measure of the rigidity of the superconducting condensate.

The generic doping dependence of the thermodynamic, electrodynamic and transport properties of high- $T_{c}$ superconductors remains a puzzle despite many years of study. Different techniques have at times produced seemingly contradictory results, leading to an increase rather than a decrease in the number of candidate theories. Their unusual behaviour is often taken to be a signature of exotic physics yet it should be related to the electronic energy-momentum dispersion, obtained for example from angle-resolved photoemission spectroscopy (ARPES). The aim of the theoretical component of 
this project was to calculate various transport and thermodynamic properties from the energy-momentum dispersion and compare the results with experimental data.

The thesis is organised as follows. Chapter 2 contains brief background information on aspects of high- $T_{c}$ superconductivity relevant to the understanding of the results presented in this work, especially those of chapter 6 . More detailed information can be found in the cited references.

Chapter 3 describes the experimental techniques employed in sample preparation, as well as a listing of the measurement apparatus employed. All measurements were performed using standard techniques on commercial lab equipment and so descriptions of how this equipment works is not included in this work. The experimental results are presented in chapter 4 .

Chapter 5 details the techniques employed in the computational part of this work. In particular, the program architectures and algorithms are described in a programming language independent manner and should be understandable by readers who are unfamiliar with computer programming. The computational results and comparison with experimental data is presented in chapter 6 .

Lastly, the summary and conclusions are presented in chapter 7.

It is our view that the present studies have firstly provided an important bridge between the thermodynamics and the band structure as probed by ARPES. Secondly, and perhaps more importantly, this work has identified the key features governing the universal phase diagram and the systematic evolution of physical properties with doping. These key features actually provide a strategy for tailoring the physical properties and, most importantly, 
for controlling the value of $T_{c}$. The puzzling but exciting problem of high- $T_{c}$ physics is certainly not yet solved but the present work clarifies many features and lights the way forward to that final goal. 


\section{Chapter 2}

\section{Background}

\subsection{Crystal Structure}

The high- $T_{c}$ superconductors are brittle copper oxide based ceramics known as cuprates. Their structure can be viewed in a simple picture as a stack of copper oxide $\left(\mathrm{CuO}_{2}\right)$ sheets extending in the ab-plane separated by charge reservoir layers. The resulting transport properties are highly anisotropic. Electron dynamics are quasi two dimensional, being largely confined to the $a b$-plane, while $c$-axis transport is weak, and for underdoped cuprates incoherent.

More specifically, the crystal structure comprises a set of $n \mathrm{CuO}_{2}$ sheets of corner-linked square-planar coordinated copper. For $n>1$, the $\mathrm{CuO}_{2}$ sheets are separated from one another by an oxygen-free plane of yttrium or calcium atoms. Generally speaking, $T_{c}$ rises with the number of $\mathrm{CuO}_{2}$ sheets in the set up to $n=3$ or 4 . For higher values the carriers doped from the charge reservoir layers are increasingly diluted and $T_{c}$ falls due to underdoping[15]. Each set of 
$\mathrm{CuO}_{2}$ sheets is separated from the next by metal-oxide layers where the metal atoms are usually lanthanum, barium, thallium or bismuth[16]. The crystal structure of the two-layer $(n=2)$ Bi-Sr-Ca-Cu-O (BSCCO) superconductor, $\mathrm{Bi}_{2} \mathrm{Sr}_{2} \mathrm{CaCu}_{2} \mathrm{O}_{8+\delta}$ (Bi-2212), is shown in Fig. 2.1. The $\mathrm{Y}-\mathrm{Ba}-\mathrm{Cu}-\mathrm{O}$ (YBCO) cuprates are further complicated by the presence of $\mathrm{CuO}$ chains extending in the $b$ direction. The structure of $\mathrm{YBa}_{2} \mathrm{Cu}_{4} \mathrm{O}_{8}$ (Y-124) is shown in Fig. 2.2.

\subsection{Phase Diagram}

The parent compounds of the high- $T_{c}$ cuprates are Mott-Hubbard antiferromagnetic insulators. Simple band structure considerations suggest a half filled $3 d_{x^{2}-y^{2}}$ band and hence a parent metallic state. But the strong on-site Coulomb repulsion causes a splitting of this band into an occupied lower Hubbard band and an empty upper Hubbard band. The undoped state is therefore an insulator. In effect, the insulating nature of these materials results from strong Coulomb repulsion which prevents electrons from hopping between neighbouring $\mathrm{Cu}$ sites. The Coulomb repulsion energy is known as the Hubbard $U$. The small amount of electron hopping, in combination with the Pauli exclusion principle insists that the spins on the $\mathrm{Cu}$ ions arrange themselves antiferromagnetically as illustrated in Fig. 2.3 in what is known as a Néel lattice.

Holes may be chemically doped into the cuprates by replacing some of the trivalent $(3+)$ ions in the charge reservoir layers with divalent $(2+)$ ions. The divalent ions supply only two electrons where three are required, and so to maintain charge neutrality electrons are depleted from the $\mathrm{CuO}_{2}$ layers. In 


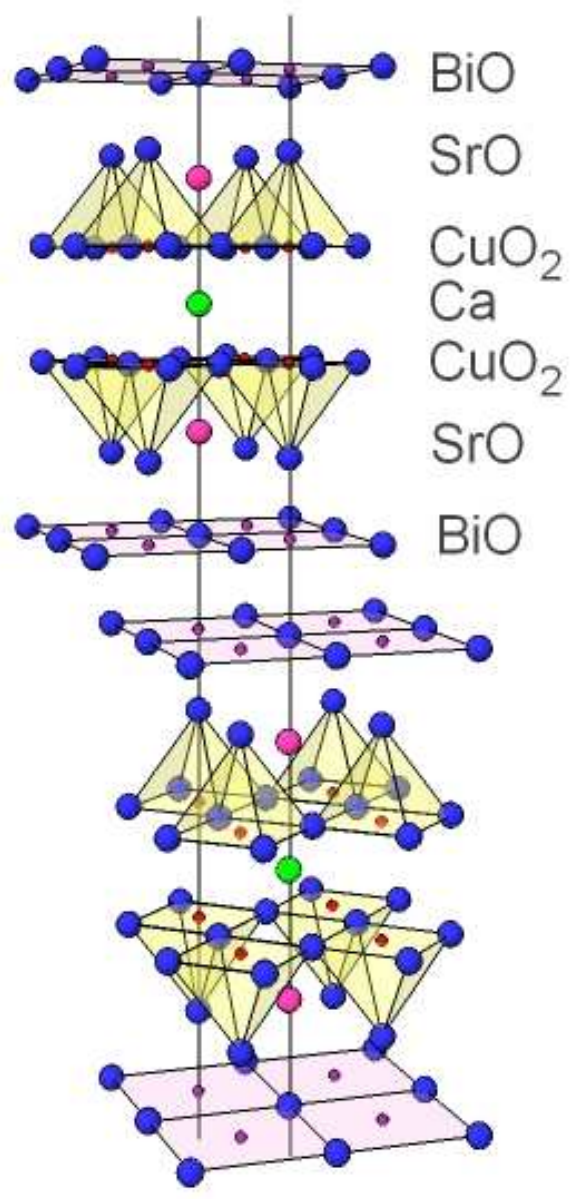

Figure 2.1: Crystal structure of Bi-2212[17]. 

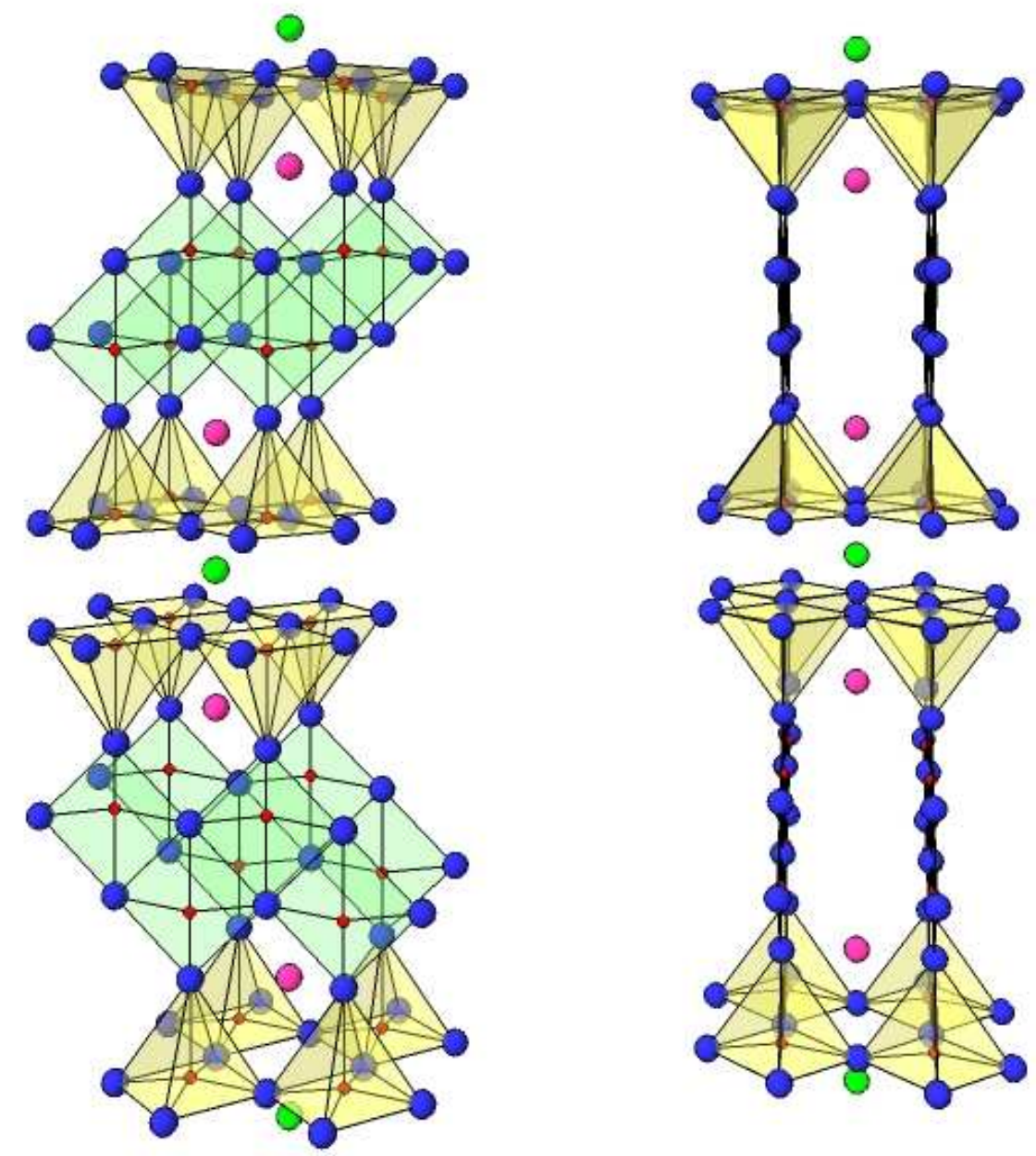

Figure 2.2: Two views of the crystal structure of Y-124[17]. Yttrium and barium atoms are shown in green and pink respectively. The double $\mathrm{CuO}$ chains are clearly visible between the opposing $\mathrm{CuO}_{5}$ square pyramid pentahedra. 


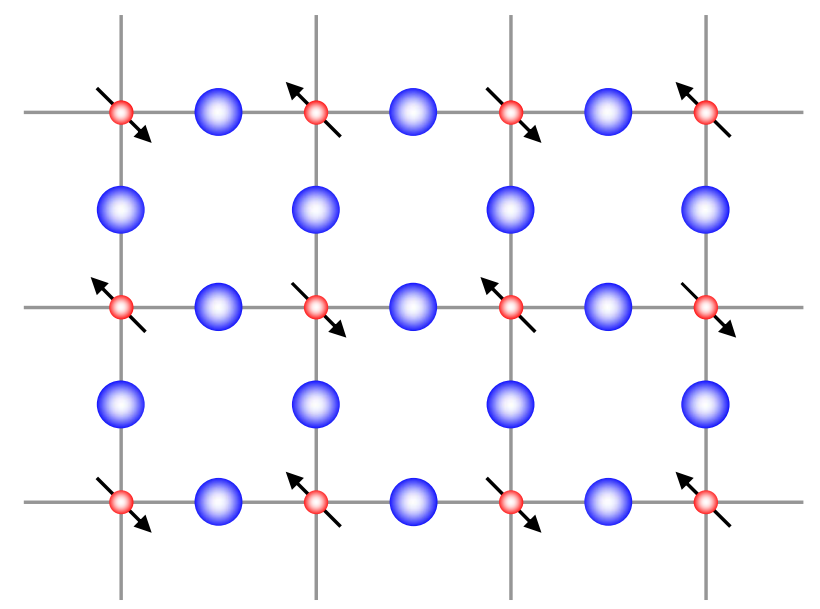

Figure 2.3: Antiferromagnetic structure of the copper oxide plane. Copper atoms are red and oxygen atoms are blue.

certain cuprates (e.g. $\mathrm{Bi}_{2} \mathrm{Sr}_{2} \mathrm{CaCu}_{2} \mathrm{O}_{8+\delta}$ ) additional holes can also be introduced by altering the oxygen content. Some cuprates, while stoichiometric, are self-doped. Examples include $\mathrm{YBa}_{2} \mathrm{Cu}_{3} \mathrm{O}_{7}$ and $\mathrm{YBa}_{2} \mathrm{Cu}_{4} \mathrm{O}_{8}$.

The hole-doped cuprates have a somewhat universal doping phase diagram, an example of which is illustrated in Fig. 2.4. The doped holes are free to hop between copper sites resulting in a melting of the Néel lattice and a corresponding drop in the Néel temperature, $T_{N}$, to zero with doping. Following the disappearance of the 3D antiferromagnetic state superconductivity emerges with a transition temperature, $T_{c}$, that has a roughly parabolic dependence on hole concentration, $p$. It has been argued that most cuprates exhibit superconductivity between hole concentrations of 0.05 and 0.27 with $T_{c}$ following the empirical relation[18] $T_{c} / T_{c, \max }=1-82.6(p-0.16)^{2}$. (An exception is the single layer $\mathrm{Bi}_{2} \mathrm{Sr}_{2} \mathrm{CuO}_{6+\delta}$ system.)

The maximum transition temperature occurs at optimal doping, $p_{\text {opt }}=$ 


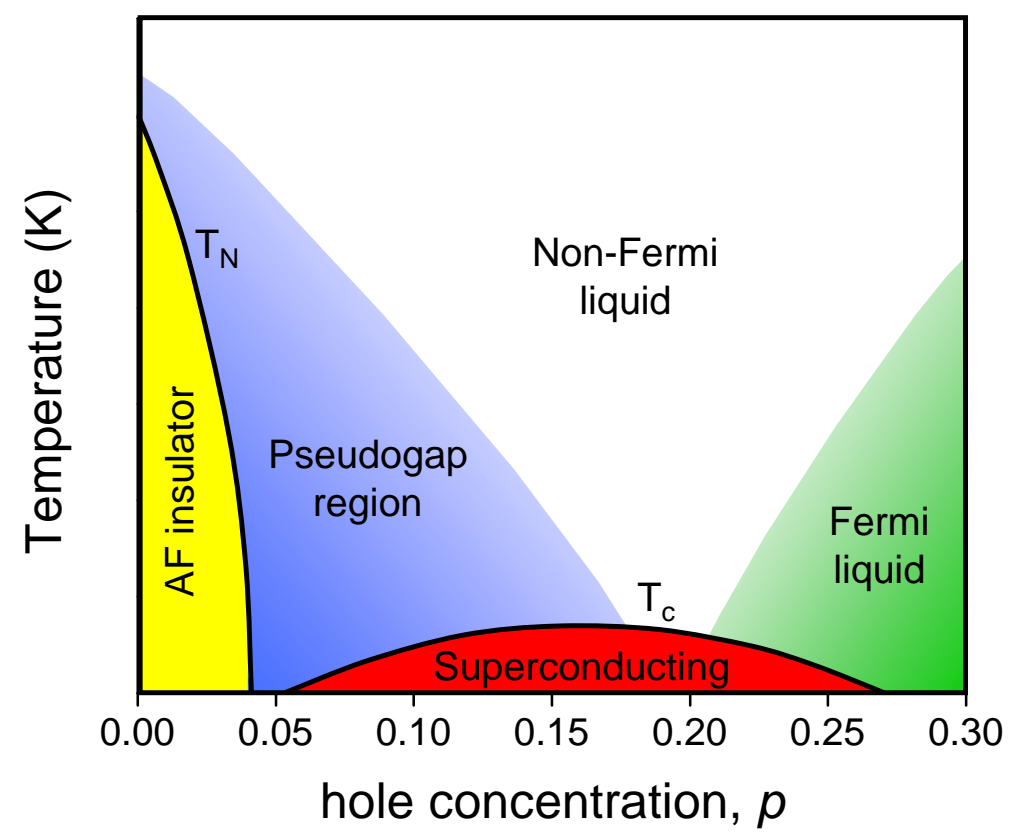

Figure 2.4: The temperature-doping phase diagram of the hole-doped high- $T_{c}$ cuprates. 
0.16. Optimal doping divides the phase diagram into underdoped $(p<0.16)$ and overdoped $(p>0.16)$ regions. The underdoped region is dominated by the pseudogap (to be discussed later in the chapter) while in the heavily overdoped region the cuprates exhibit Fermi-liquid-like metallic behaviour. The intermediate region is characterised by a non-Fermi-liquid regime in which the behaviour of physical properties deviate from those expected in a Landau Fermi liquid picture. The most notable example is the linear temperature dependence of the in-plane electrical resistivity. Varma et al.[19] have proposed a 'marginal-Fermi-liquid' phenomenology to describe the behaviour in this regime.

\subsection{Angle Resolved Photoemission Spectroscopy}

Angle resolved photoemission spectroscopy (ARPES) uses the photoelectric effect to determine the electronic structure of materials. The geometry of a typical ARPES measurement is shown in Fig. 2.5. Incident high energy photons with energy $h \nu$ eject electrons from the sample. The energies of the emitted electrons are measured by an electron analyser as a function of polar $(\theta)$ and azimuthal $(\phi)$ angles. Using energy and momentum conservation laws the initial energy and momentum of the electron, and by extension the electronic energy-momentum dispersion of the sample, can be deduced. Energy dispersion curves (EDC's) can be produced from energy scans at fixed momentum, and momentum distribution curves (MDC's) can be produced 
from momentum scans at fixed energy.

The photons used in ARPES experiments on HTS materials have typically been supplied by a synchrotron light source with energies in the ultraviolet $(<100 \mathrm{eV})$ range. At these energies ARPES measurements are extremely surface sensitive. To achieve measurements representative of the bulk requires atomically clean samples. As a result the majority of ARPES studies on the cuprates have been performed under ultra high vacuum conditions on BSCCO single crystals which can be naturally cleaved in situ between the $\mathrm{BiO}$ planes. Most recently an ARPES system has been developed in which a $6 \mathrm{eV}$ laser is used as the photon source[20]. The use of lower energy photons offers the advantages of increased bulk sensitivity, reduced background signal and increased energy and momentum resolution. These advantages might allow the ARPES technique to be extended to materials that do not cleave easily.

Some notable contributions from ARPES to the understanding of the high- $T_{c}$ cuprates include the mapping of the Fermi surface[21], the observation of an extended saddle-point singularity in the dispersion $[22,23]$, the observation of a normal-state pseudogap [24, 25] and related break up of the Fermi surface in the underdoped regime[26], and the observation of an anisotropic superconducting gap consistent with a $d$-wave order parameter[27, $28,29,30]$. 


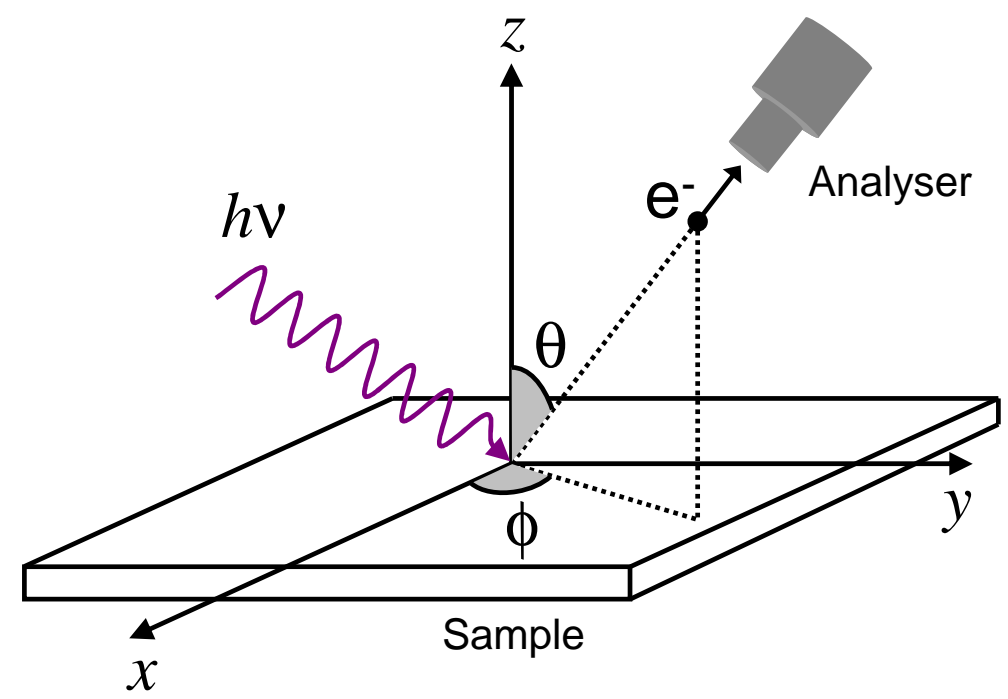

Figure 2.5: ARPES measurement geometry. A photon with energy $h \nu$ ejects an electron $e^{-}$that is detected by an analyser.

\subsection{Energy-momentum Dispersion}

The electronic structure of the cuprates is often interpolated from experimental data using a semi empirical tight binding model. In the tight binding model the electron wavefunction is approximated by a linear combination of isolated atomic orbitals. The model gives good qualitative results for bands that are derived from strongly localised atomic orbitals. The energy-momentum dispersion that results from the tight binding model is parametrized by hopping parameters. These parameters describe the ability of an electron to jump from one specific atom to another and are obtained from fits to experimental data. The relationship between the electron orbitals in the $\mathrm{CuO}_{2}$ plane and the hopping parameters is illustrated in Fig. 2.6.

Most theories of high-temperature superconductivity are based on the 


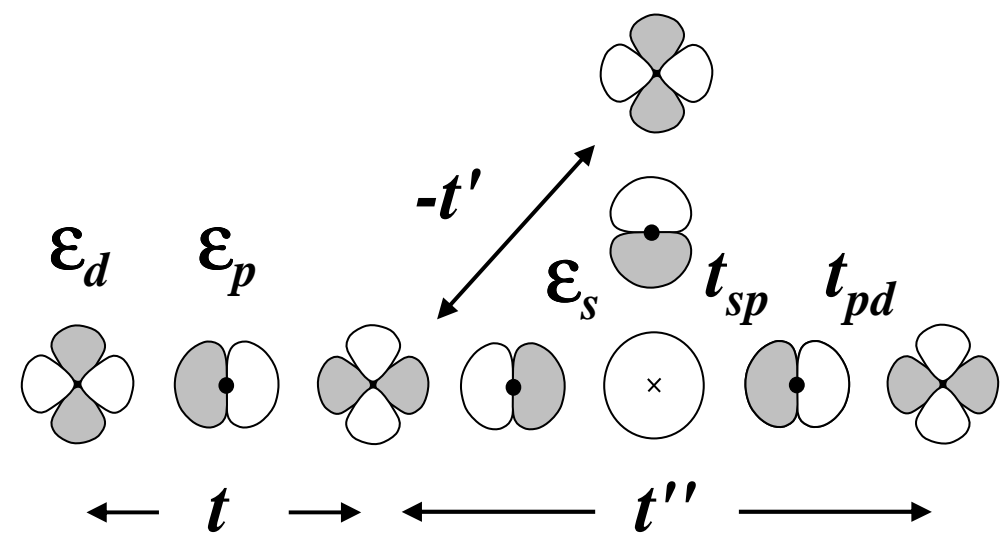

Figure 2.6: Schematic diagram of electron orbitals in the $\mathrm{CuO}_{2}$ plane and related hopping parameters (adapted from Ref. [31]).

Hubbard model with one $\mathrm{Cu} d_{x^{2}-y^{2}}$-like orbital per $\mathrm{CuO}_{2}$ unit. The single electron energy-momentum dispersion in this model given by[31]

$$
\epsilon(\mathbf{k})=-2 t\left(\cos k_{x}+\cos k_{y}\right)+4 t^{\prime} \cos k_{x} \cos k_{y}-2 t^{\prime \prime}\left(\cos 2 k_{x}+\cos 2 k_{y}\right)+\ldots
$$

where $t, t^{\prime}, t^{\prime \prime}, \ldots$ denote the hopping integrals on the square lattice.

The six-parameter Bi-2212 energy-momentum dispersion reported by Norman et al.[32] is plotted in Fig. 2.7. It was obtained from tight-binding fits to ARPES data. This dispersion does not include fine structure due to renormalization effects or bilayer splitting. Although the exact values of the hopping parameters differ between the cuprates the overall shape of the dispersion is common. It is dominated by saddle points at $( \pm \pi, 0)$ and $(0, \pm \pi)$ which give rise to a van Hove singularity in the density of states. 


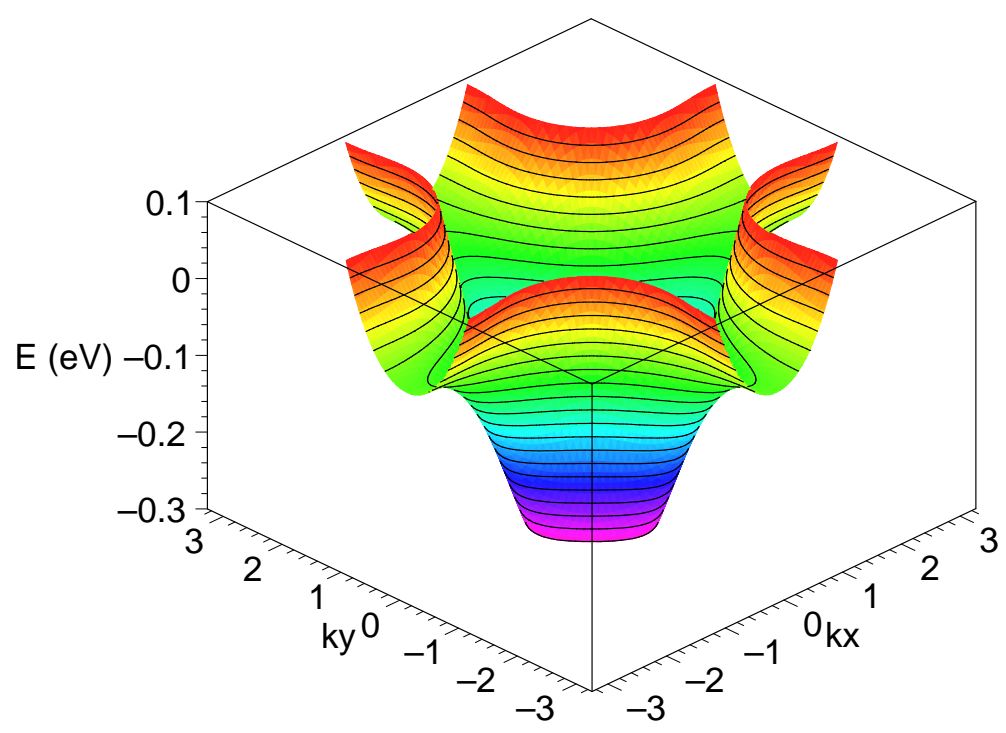

Figure 2.7: Energy momentum dispersion of Bi-2212 determined from a six parameter tight-binding fit to ARPES data[32]. $k_{x}$ and $k_{y}$ are in units of $1 / a$ where $a$ is the lattice spacing.

\subsubsection{Fermi Surface}

In the present work the dispersion is assumed to be completely two-dimensional. In two dimensions the Fermi 'surface' is defined by a constant energy contour. Figure 2.8 shows the dispersion projected onto the $\left(k_{x}, k_{y}\right)$ plane with the Fermi surface near optimal doping indicated by the magenta coloured constant energy contour. The Fermi surface is centered on the $(\pi, \pi)$ point and is called 'hole-like' because it encloses unfilled or empty states[33].

ARPES measurements on the single layer $\mathrm{Bi}_{2} \mathrm{Sr}_{2} \mathrm{CuO}_{6+\delta}$ (Bi-2201) material show that the dispersion is, to a good approximation, rigid with doping[34]. In other words, the shape of the dispersion is independent of doping. The position of the Fermi energy does however change with doping, progressing down the energy contours in Fig. 2.7 or towards $\mathbf{k}=(0,0)$ in Fig. 2.8. As 


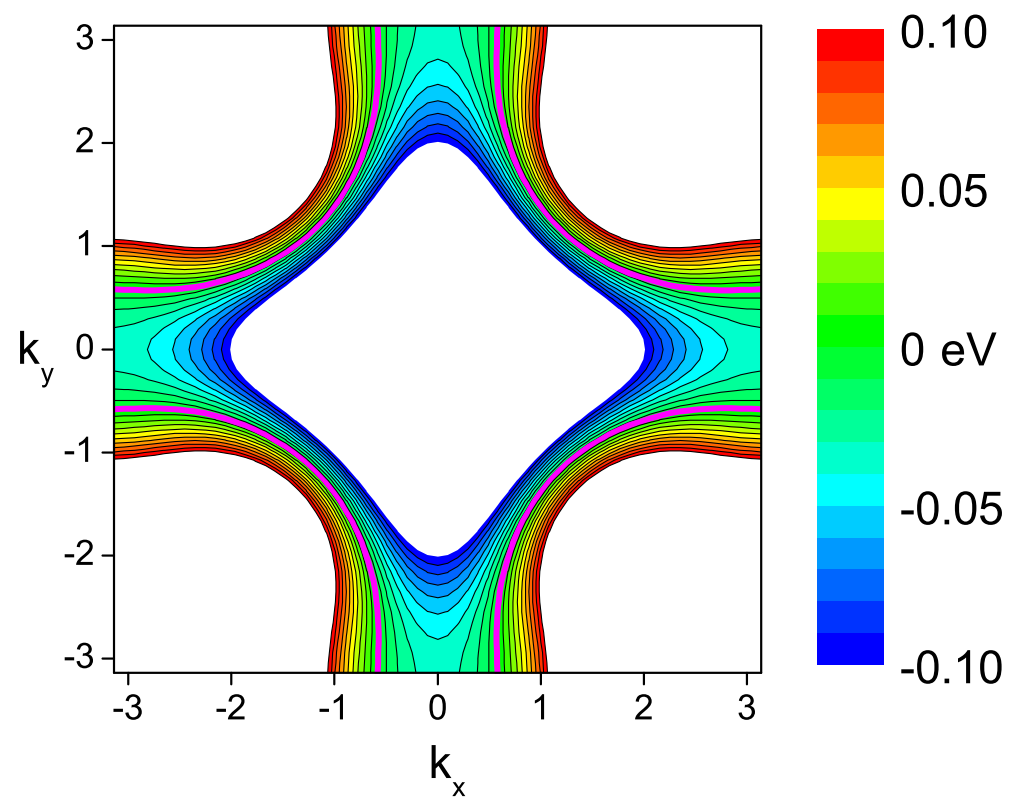

Figure 2.8: Energy momentum dispersion of Bi-2212 from Fig. 2.7 projected onto the $\left(k_{x}, k_{y}\right)$ plane. The Fermi surface near optimal doping is indicated by the magenta coloured constant energy contour. 

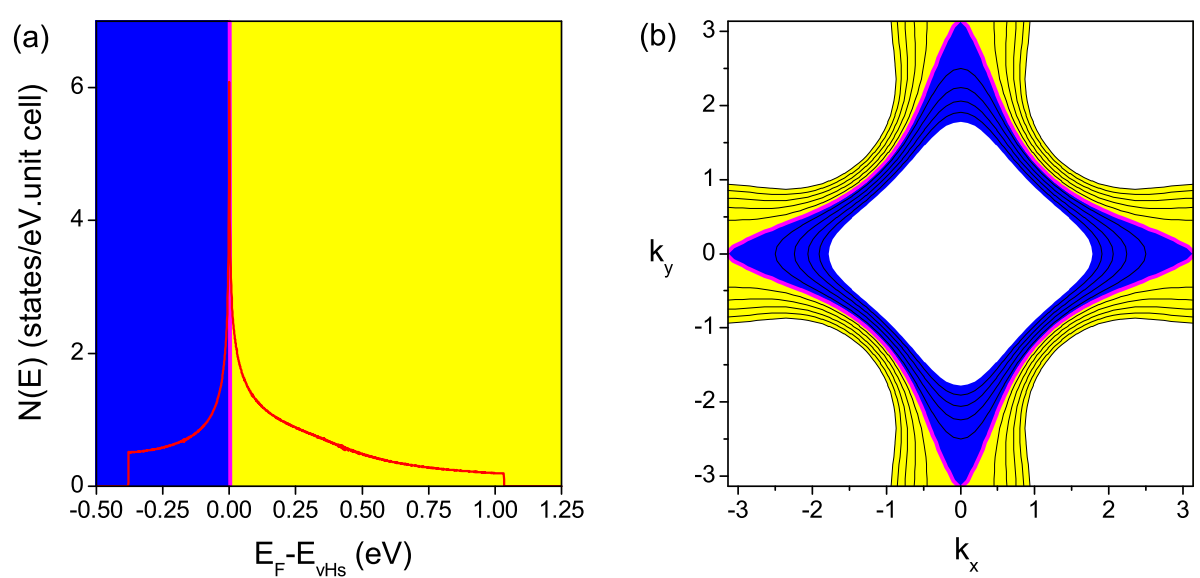

Figure 2.9: The van Hove singularity in the density of states shown in (a) denotes the boundary between hole-like (yellow) and electron-like (blue) Fermi surfaces shown in (b).

can be seen in Fig. 2.9 the van Hove singularity (vHs) denotes the boundary between hole-like and electron-like Fermi surfaces. Maps of the Fermi surface of Bi-2201 from ARPES[34] show that the topology of the Fermi surface evolves smoothly from hole-like to electron-like as doping is increased. This implies that as a function of increasing doping the Fermi energy sweeps from high energy to low energy and indeed passes through the vHs.

\subsubsection{Bilayer Splitting}

The bilayer Bi-2212 cuprate contains two $\mathrm{CuO}_{2}$ planes per unit cell which are separated by a layer of calcium atoms. Various calculations $[35,36,37]$ of the band structure of bilayer cuprates predicted that intra bilayer coupling would lead to a splitting of the band into an upper antibonding band and a lower bonding band. This so-called bilayer splitting was only observed recently[38, 39] by ARPES after improvements in measurement resolution and the ability 
to prepare heavily overdoped Bi-2212 samples had been developed. The strong dependence of the relative intensities of bonding and antibonding band spectra on the incident photon energy was found to be responsible for previous conflicting observations of hole-like[40] and electron-like[41] Fermi surfaces in similarly doped samples.

Figure 2.10 shows the bilayer energy momentum dispersion of Bi-2212 determined from tight-binding fits to high-resolution ARPES data[42]. The bonding band dispersion is shown in blue and the antibonding band dispersion in red. The splitting in momentum space is greatest in the antinodal $[( \pm \pi, 0)$ and $(0, \pm \pi)]$ regions and small or non existent along the nodal $[(0,0) \rightarrow(\pi, \pi)]$ symmetry line reflecting the symmetry of the intra-plane hopping parameter $t_{\perp}[43]$. The maximum energy splitting is of the order of 100meV. Clearly, with increasing doping, the descending Fermi level will first cross the antibonding vHs then the bonding band vHs.

\subsubsection{Superconducting Gap}

The superconducting state is characterized by an energy gap in the density of states related to the binding energy of the Cooper pairs. In conventional low-temperature BCS superconductors the Cooper pairs are formed from electrons with equal and opposite momentum and so have zero net momentum[44, 3]. This results in an isotropic or $s$-wave symmetric k-space energy gap. In contrast, ARPES[27, 28], penetration depth[45], Raman spectroscopy[46] and nuclear magnetic resonance[47] experiments on the cuprates indicate the presence of an anisotropic superconducting gap consis- 


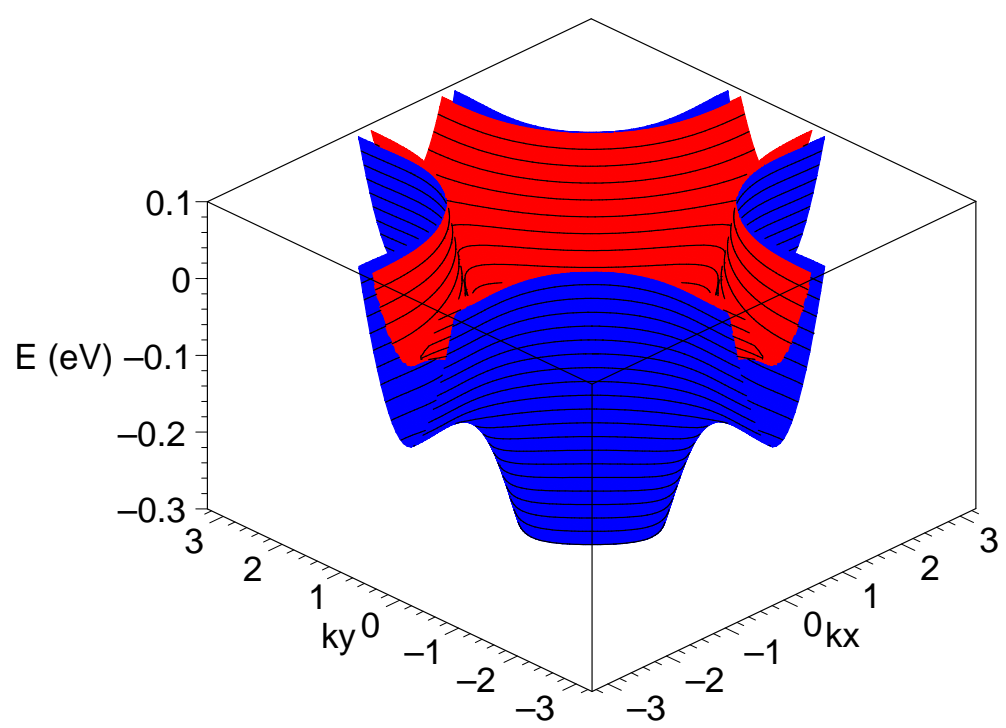

Figure 2.10: Energy momentum dispersion, including the effect of bilayer splitting, of Bi-2212 determined from tight-binding fits to high-resolution ARPES data[42]. The bonding (antibonding) band dispersion is shown in blue (red).

tent with $d$-wave symmetry, in which the Cooper pairs have non-zero momentum. The above techniques reveal information about the magnitude of the order parameter but not its sign, and it was not until phase sensitive measurements were performed that the now generally accepted $d_{x^{2}-y^{2}}$ sym-

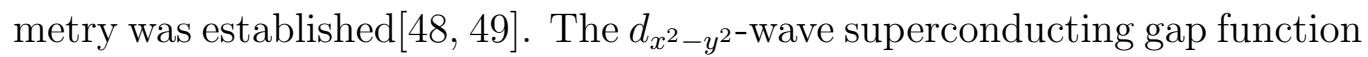
$\Delta_{0} \frac{1}{2}\left(\cos k_{x}-\cos k_{y}\right)$ is plotted in Fig. 2.11(a). The gap function has line nodes extending along the zone diagonals, which give rise to four point nodes in the superconducting state [two of which are illustrated in Fig. 2.11(b)], and antinodes at $( \pm \pi, 0)$ and $(0, \pm \pi)$. 


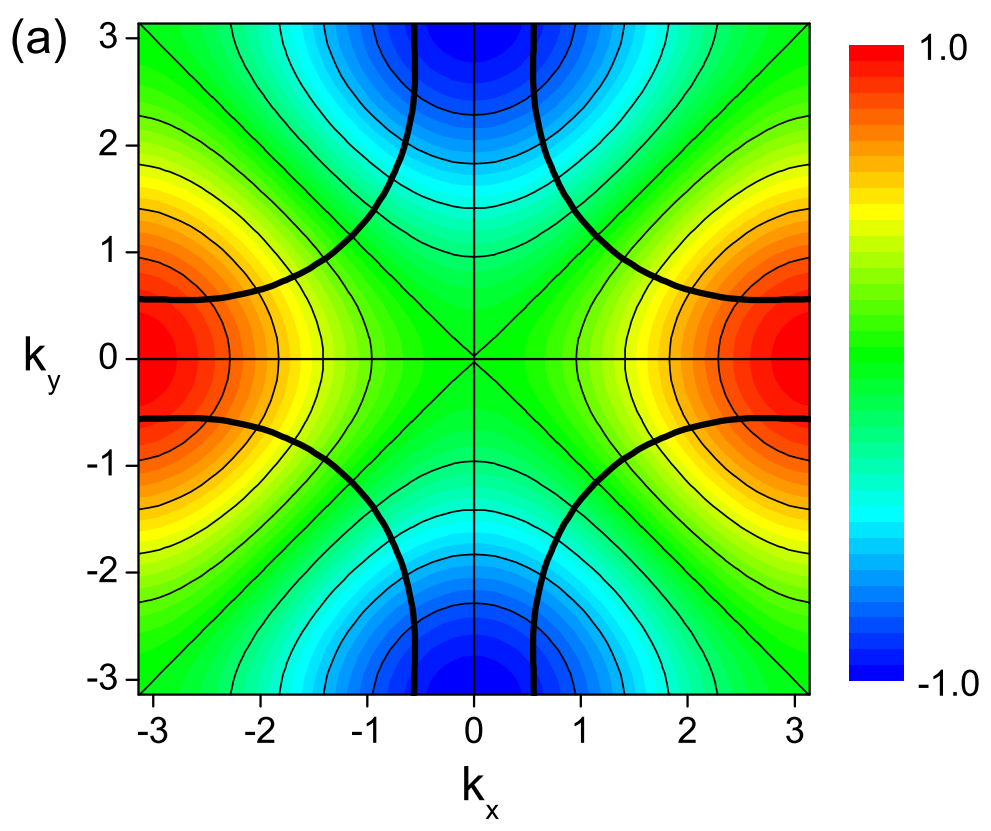

(b)

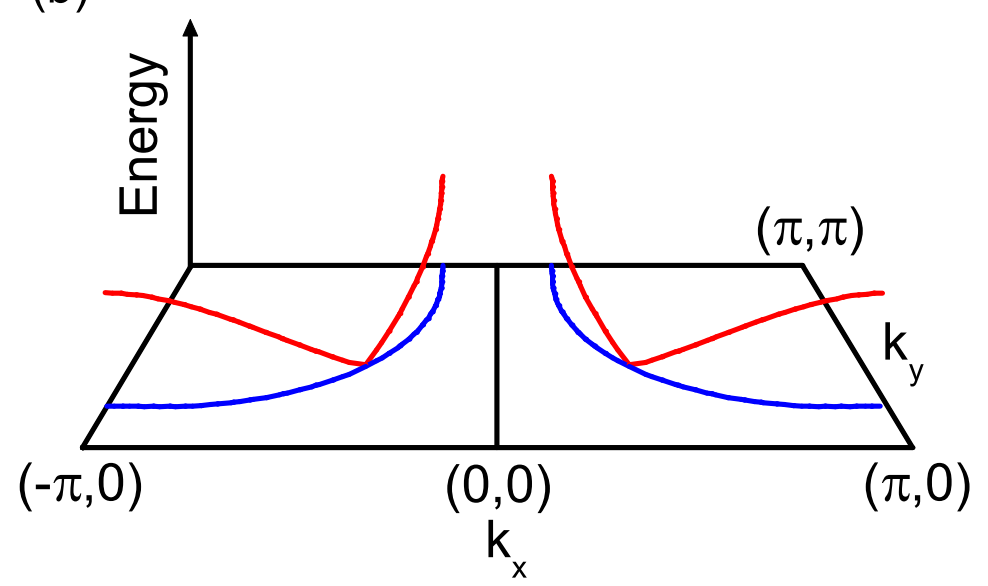

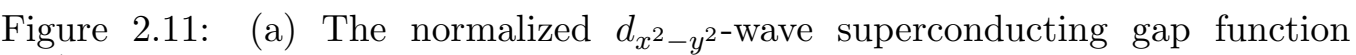
$\Delta_{0} \frac{1}{2}\left(\cos k_{x}-\cos k_{y}\right)$. The thick black lines denote the Fermi surface. (b) In the superconducting state the $d$-wave gap opens up on the Fermi surface (blue) resulting in point nodes on the zone diagonals. The same size nodal gap also opens up in the occupied states below the Fermi level. 


\subsection{Van Hove Scenario}

In BCS theory[3] the superconducting transition temperature, $T_{c}$, is exponentially related to $-1 / N\left(E_{F}\right)$ where $N\left(E_{F}\right)$ is the density of states at the Fermi level. The observation by ARPES of saddle points[50, 22, 23] (flat regions) in the dispersion implies the existence of a van Hove singularity (vHs) in the density of states. Initially these observations lent weight to theories based on the so-called 'van Hove scenario'. For a review of the van Hove scenario see Ref.[51]. Under this scenario the peak in $T_{c}$ as a function of hole concentration is attributed to the Fermi level sweeping through the vHs at optimal doping[52]. Other properties explained under the van Hove scenario included the doping dependence of the isotope effect on $T_{c}[53]$, thermoelectric power[54] and superconducting gap anisotropy[55]. The vHs itself was even proposed as the basis of a novel electronic pairing mechanism[56].

A major problem with the van Hove scenario is that ARPES measurements indicate that the Fermi level crosses the vHs not at optimal doping but in the very heavily overdoped region of the phase diagram. In the case of Bi2201 the crossing is near where $T_{c}$ goes to zero[34], while at optimal doping the vHs lies $42 \mathrm{meV}$ below the Fermi level[57]. A similar situation is observed in La-214[58, 59, 60, 61]. Measurements performed on Bi-2212[42] indicate that Fermi level crosses the antibonding band vHs near $p=0.22$ where $T_{c}$ is well below $T_{c, \max }$ but still quite high at around $60 \mathrm{~K}$. The consequences of the Fermi level crossing the vHs in the overdoped regime are explored as part of this work. One has to ask, for example, why is there no local peak in the $p$-dependence of $T_{c}$ which occurs at the vHs crossing? 


\subsection{Pseudogap}

The underdoped region of the phase diagram is dominated by a partial gap in the density of states known as the pseudogap, which opens up in the normal state. For reviews see Refs. [62] and [63]. First observed as a suppression of the ${ }^{63} \mathrm{Cu}$ nuclear relaxation rate[64] and static spin susceptibility[65, 66] the pseudogap was initially interpreted as a 'spin gap'. Electronic specific heat measurements by Loram et al[67,68]. revealed the pseudogap to be a gap in the quasiparticle density of states. This finding was subsequently confirmed by ARPES[24, 25] and tunneling experiments[69, 70, 71].

In ARPES the pseudogap becomes observable below a temperature $T^{*}$ and first opens up at the antinodal $( \pm \pi, 0)$ and $(0, \pm \pi)$ regions of the Fermi surface, resulting in ungapped portions of the Fermi surface known as Fermi $\operatorname{arcs}[26,72]$. The Fermi arcs shrink with decreasing temperature as the pseudogap extends towards the nodal regions of the Fermi surface. The magnitude of the pseudogap at the antinodes is observed to be constant implying that the pseudogap 'fills in' with increasing temperature, in contrast to the superconducting gap which 'closes' with increasing temperature[73, 26]. This filling behaviour has also been inferred from tunneling[69] and fits to the spin susceptibility[74]. The ARPES data has led to the widespread belief that the pseudogap fills completely at $T^{*}$ resulting in the full restoration of the Fermi surface above $T^{*}$. As part of this work this view is explicitly shown to be inconsistent with thermodynamic data and is most likely an artifact of the way that the ARPES data is interpreted. As such, the $T^{*}$ line on the phase diagram represents the energy scale of the pseudogap or a crossover rather 
than a true phase transition. Although it is agreed that the magnitude of the pseudogap increases monotonically with decreasing doping, the exact form of the $T^{*}$ line on the phase diagram depends on the theoretical model used to describe the pseudogap[63].

The origin of the pseudogap is one of the most highly debated topics in the field of HTS. Some believe that solving this problem will provide a key to unlocking the mystery of high temperature superconductivity in the cuprates. Regardless of whether this turns out to be true or not, the pseudogap presents a significant hurdle in the race to the finish line. The numerous theoretical models for the pseudogap fall roughly into two camps: those which attribute the pseudogap to preformed pairs; and those which attribute the pseudogap to an, as yet, unknown correlation that competes with superconductivity.

In the preformed pairs scenario the pseudogap arises due to the formation of Cooper pairs above $T_{c}$ that lack long-range phase coherence. The pseudogap phase is then essentially a precursor of the superconducting state. At $T_{c}$ the pairs become phase coherent and superconductivity is achieved. Under this scenario the pseudogap merges smoothly into the superconducting gap. The phase diagram is dominated by a single energy scale, namely the antinodal gap magnitude, and the $T^{*}$ line is coincident with $T_{c}$ on the overdoped side [see Fig. 2.12(a)]. Experimental evidence cited in support of this scenario includes the similarity of the momentum dependence and magnitude of the pseudogap to the $d$-wave superconducting gap, as determined in early ARPES experiments[24, 25], and the apparent smooth evolution of the pseudogap into the superconducting gap[70].

In the second scenario the correlation underlying the pseudogap is in- 
dependent and competes with that which gives rise to superconductivity. The pseudogap is separate from the superconducting gap and removes states which, in its absence, would otherwise be available for superconductivity. In this scenario the $T^{*}$ line drives through the superconducting dome vanishing at a zero temperature quantum critical point at $p_{\text {crit }} \approx 0.19$ [see Fig. 2.12(b)]. Experimental evidence indirectly supporting this picture includes the rapid collapse of various properties below critical doping including the superfluid density, electronic entropy, condensation energy, critical current density and irreversibility field[75]. The most direct signature of this scenario is the observation of two distinct energy gaps in underdoped cuprates corresponding to the pseudogap and superconducting gap. Two gaps have been observed more recently by Andreev reflection[76], Raman spectroscopy[77], and most recently by ARPES[78, 79] and scanning tunneling microscopy[80], seriously eroding the support for the preformed pairs scenario. The two gap scenario was inferred much earlier from specific heat $[67,68]$ and NMR Knight shift[81] data. Further evidence that the pseudogap is unrelated to superconductivity comes from the observation of a very similar pseudogap state in the colossal magnetoresistive bilayer manganite $\mathrm{La}_{1.2} \mathrm{Sr}_{1.8} \mathrm{Mn}_{2} \mathrm{O}_{7}[82]$.

\subsection{Isotope Effect}

Observations of the predicted linear dependence of $T_{c}$ on the inverse square root of the isotopic mass in tin[14] helped confirm the virtual exchange of phonons as the source of electron pairing in conventional low-temperature superconductors. The role of phonons in HTS remains unclear as the results 


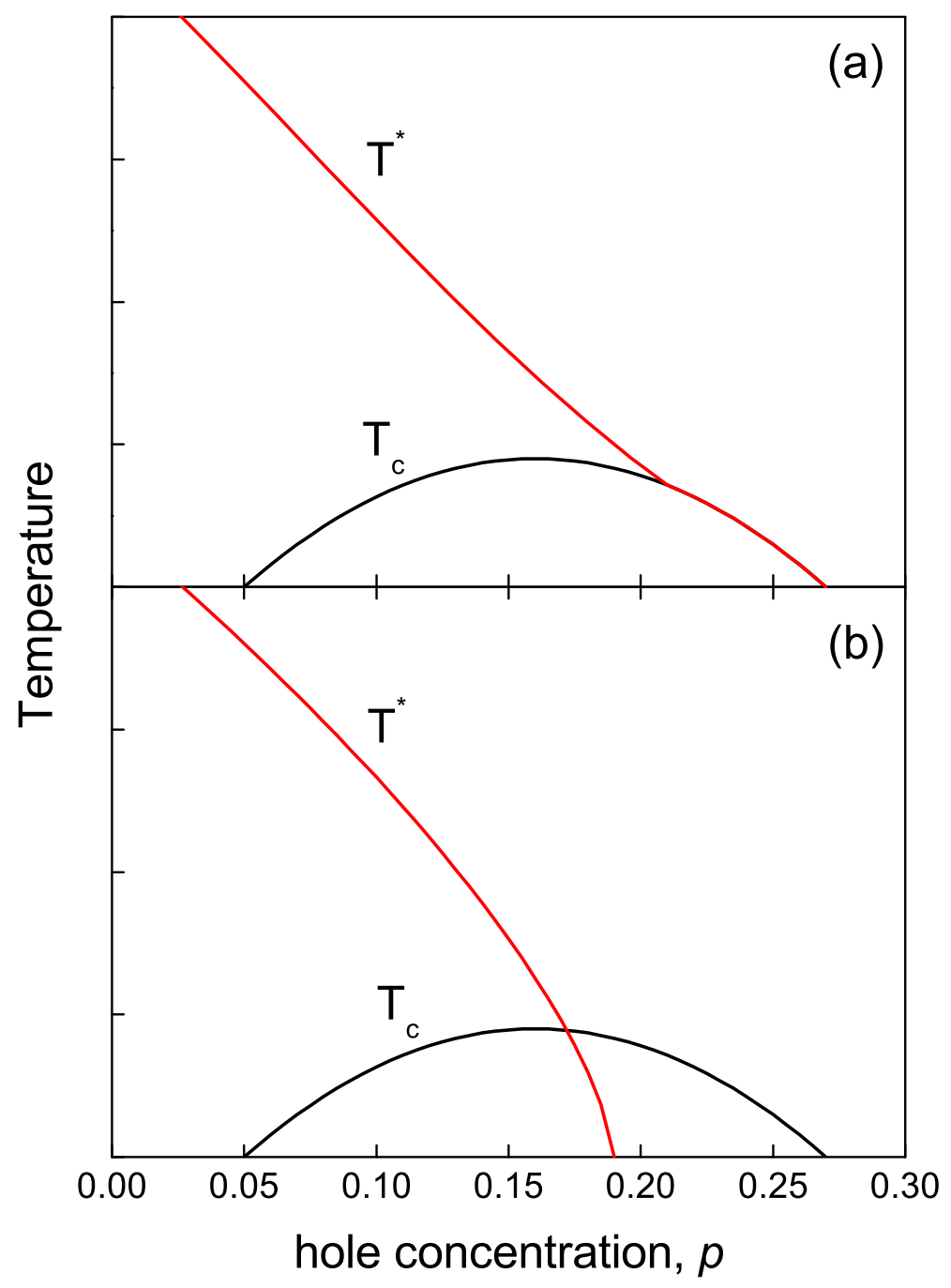

Figure 2.12: (a) In scenarios based on preformed pairs $T^{*}$ merges smoothly onto the $T_{c}$ curve in the overdoped region. (b) In the competing correlation scenario $T^{*}$ drives through the $T_{c}$ dome, vanishing at critical doping $p_{\text {crit }}=0.19$. 
of isotope effect experiments performed on the cuprates are not as easy to interpret. The isotope exponent $\alpha(E)$ in a given property $E$ is defined as $\alpha(E)=-(\Delta E / E) /(\Delta M / M)$ where $M$ is the isotopic mass. For BCS superconductors $\alpha \approx-0.5$. In the LSCO and YBCO systems the isotope effect in $T_{c}, \alpha\left(T_{c}\right)$, is found to be strongly dependent on doping. In $\operatorname{YBCO}[83,84]$ $\alpha\left(T_{c}\right)$ increases rapidly from a value of around 0.06 at optimal doping to values exceeding 0.5 in the deeply underdoped regime. A similar evolution is observed in the LSCO system $[85,86,87,88]$ but, in addition to the rise with underdoping, $\alpha\left(T_{c}\right)$ exhibits a local maximum at $p=0.125$ which has been attributed to the presence of spin-charge ordering known as "stripes" at this doping[89].

An attempt to explain the rise of the isotope effect coefficient with underdoping was made by Tsuei et al.[53] under the van Hove scenario. In their model the vHs is located at the Fermi level at optimal doping and is responsible for the maximal value of $T_{c}$ there. Their calculated isotope effect coefficient has a minimum at optimal doping and increases symmetrically about this point as a function of doping. However, this symmetrical behaviour is not observed in the data[84].

In metals the energy-momentum dispersion becomes renormalized due to screening by the ions[90]. This electron-phonon correction only affects the dispersion close to the Fermi level on the scale of the Debye energy $\left(\hbar \omega_{D}\right)$ resulting in a kink in the dispersion. The kink separates the dispersion into a low energy part that is close to the Fermi level and a high energy (unrenormalized) part that is further from the Fermi level. ARPES has revealed the presence of such a kink in the cuprate nodal dispersion at around $70 \mathrm{meV}$ 
below the Fermi level[91, 92, 93]. Several excitations have been advanced to explain the origin of the kink including, but not limited to, phonons[93], and a collective magnetic mode[94]. Detailed momentum dependent measurements indicate that the kinks in the nodal and antinodal regions behave differently with temperature, suggesting that they may arise from different mechanisms[95]. Cuk et al[96]. and Devereaux et al[97] have interpreted the nodal kink in terms of the in-plane $\mathrm{Cu}-\mathrm{O}$ breathing mode and the antinodal kink in terms of the $B_{1 g}$ oxygen bond-buckling phonon. The slope of the high energy part of the nodal kink is observed to increase with underdoping [93, 98] while the constant slope of the low energy part reveals a universal nodal Fermi velocity[98].

In 2004 Gweon et al. [99] reported an unusual isotope effect in the dispersion of Bi-2212. An unexpected and large isotope shift in the slope of the high energy part of the dispersion was found in addition to a more conventional $5 \mathrm{meV}$ shift in the kink energy. Also found was a sign reversal of the isotope shift in the high energy slope in going from the nodal to antinodal regions of the Fermi surface. However, more recent measurements reported by Douglas et al.[100] paint a different picture. Using low energy photons $(7 \mathrm{eV})$ that give extremely high resolution as well as increased bulk sensitivity they report an insignificant isotope shift in the high energy slope. The results obtained by Gweon et al. are attributed to sample misalignments, the precise control over which was found to be crucial to obtain repeatable energy positions in the high energy regime. At the 2007 March meeting of the American Physical Physical Society[101] Douglas presented further results showing a $3 \mathrm{meV}$ isotope shift in the kink energy consistent with the isotope 
induced Raman shift observed on the same samples. We await upcoming measurements for confirmation of this more conventional effect - more conventional because the bosonic energy scale is shifted while the presumably unrenormalized high energy slope is not altered.

\subsection{Superfluid Density}

Technically speaking superconductors are not perfect conductors but are perfect diamagnets. When a superconductor is cooled below its transition temperature in an applied field it becomes diamagnetic and the magnetic flux is expelled from its interior. This is known as the Meissner effect. The flux exclusion is due to surface layer screening currents that are induced as the sample is cooled below $T_{c}$. The layer of current must have a finite thickness otherwise the current density would be infinite. As a result the flux density does not fall abruptly to zero at the boundary of the superconductor but decays exponentially within the region of the screening currents. A measure of this depth is called the London penetration depth $\lambda$.

In conventional superconductors $\lambda=\sqrt{m / \mu_{0} n_{s} e^{2}}[44]$ where $n_{s}$ is the carrier density. The superfluid density, defined as $\rho_{s}=1 / \lambda^{2}$, is a measure of the rigidity of the superconducting condensate. The anisotropic structure of the high- $T_{c}$ cuprates gives rise to significant anisotropy in the $\lambda$. Though strictly unphysical, it is convenient to express this as an anisotropy in $\rho_{s}$.

In contrast to BCS superconductors the high- $T_{c}$ cuprates have been found to exhibit an isotope effect in $\rho_{s}$ (for example see Refs. [102, 103, 87]). The isotope effect in $\rho_{s}$ is observed in magnetic measurements as a reduction in 
diamagnetic screening, or equivalently, an increase in the magnetic penetration depth. The origin of this effect is still controversial. According to the expression for $\lambda$ given above an isotope effect could be seen as due to either a shift in $n_{s}$ and/or the effective mass $m^{*}$. The isotope effect in $\rho_{s}$ interpreted in terms of $m^{*}$ has been claimed as evidence for polaronic charge carriers[104]. However, a more recent study[87] was able to explain the isotope effect in $\rho_{s}$ as being due to the presence of a normal-state pseudogap that competes with superconductivity. 


\section{Chapter 3}

\section{Experimental Techniques}

The aim of the experimental part of this project was to determine the isotope effect in the separate components of the penetration depth tensor from AC susceptibility measurements on powders of $\mathrm{YBa}_{2} \mathrm{Cu}_{4} \mathrm{O}_{8}$ biaxially-aligned in epoxy. $\mathrm{YBa}_{2} \mathrm{Cu}_{4} \mathrm{O}_{8}$ was chosen because of its fixed oxygen stoichiometry. The experimental techniques employed are detailed in this chapter.

\subsection{Preparation of $\mathrm{YBa}_{2} \mathrm{Cu}_{4} \mathrm{O}_{8}$}

Polycrystalline samples of $\mathrm{YBa}_{2} \mathrm{Cu}_{4} \mathrm{O}_{8}(\mathrm{Y}-124)$ were prepared using a conventional solid state reaction technique. Stoichiometric mixtures of high purity $\mathrm{Y}_{2} \mathrm{O}_{3}(99.99 \%), \mathrm{BaCO}_{3}(99.98 \%)$ and $\mathrm{CuO}(99.99 \%)$ from Aldrich were thoroughly ground together with an agate pestle and mortar, pressed into pellets, and calcined in air at $925^{\circ} \mathrm{C}$ for 18 hours. With the aim of preparing a more homogeneous starting material the pellets were then ground up, repressed and refired at $930^{\circ} \mathrm{C}$ for 24 hours. To obtain the 124 phase the 
pellets were ground, repressed and sintered in a sequence of overnight firings at $930,935,940$ and $950^{\circ} \mathrm{C}$ under a 60 bar static oxygen atmosphere. At the end of the $950^{\circ} \mathrm{C}$ step the samples were cooled to $700^{\circ} \mathrm{C}$ over 6 hours before being cooled to room temperature over a further 6 hours.

\subsection{Oxygen Isotope Exchange}

Substitution of ${ }^{16} \mathrm{O}$ for ${ }^{18} \mathrm{O}$ was achieved by annealing a pellet in an ${ }^{18} \mathrm{O}$ enriched atmosphere at $350^{\circ} \mathrm{C}$. A control sample placed in an ${ }^{16} \mathrm{O}$ atmosphere was annealed in parallel. A schematic diagram of the oxygen isotope exchange rig is given in Fig. 3.1. The rig comprises two quartz tubes in which the samples are placed and relevant plumbing for evacuating and charging the tubes with the ${ }^{16} \mathrm{O}(>99.7 \%) /{ }^{18} \mathrm{O}(99 \%)$-enriched gasses.

Because the atmospheres are stagnant the tubes must be withdrawn from the furnace and the gas refreshed under ambient conditions. Refreshment of the gas was performed at the beginning and end of each work day for the duration of the exchange. The number of 'hits' required is a function of the number of moles of ${ }^{18} \mathrm{O}$ in the quartz tube, $N_{T}$, and the initial number of moles of ${ }^{16} \mathrm{O}$ in the sample, $N_{S}$. The exchange percentage after the $i$ th hit is given by

$$
100\left(1-\frac{N_{s}}{\left(N_{s}+N_{T}\right)}\right)^{i}
$$

The isotope exchange can be confirmed in the samples from the change in sample mass and also by the measuring shift of the oxygen vibrational modes in the Raman spectra. 


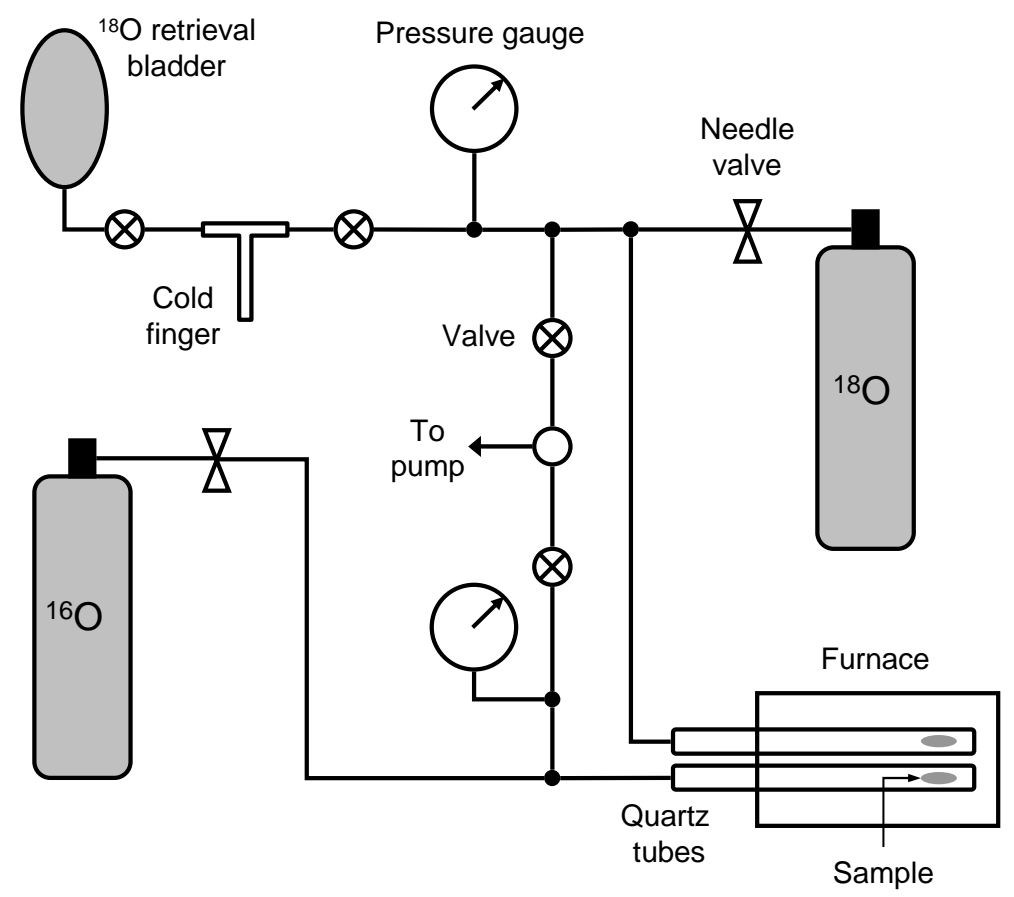

Figure 3.1: Schematic diagram of the oxygen isotope exchange rig. 
The rig includes a quartz cold finger for retrieving the gas from hits near the end of the exchange process which contains mostly expensive ${ }^{18} \mathrm{O}$. The gas can be condensed inside the liquid-nitrogen-cooled finger and stored in a collection bladder for future use.

\subsection{Biaxial Alignment}

The large anisotropy in the magnetic susceptibility of the cuprates has long been exploited to produce grain-aligned samples[105]. Typically fine grains of superconducting powder are dispersed in an epoxy or wax which is left to set in the presence of a static magnetic field. The resulting uniaxially (c-axis) aligned samples allow the separate study of properties both parallel and perpendicular to the $a b$-plane.

In this work we employed the technique pioneered in house by Staines et al.[106] to produce biaxially textured samples. The technique takes advantage of the difference in the normal-state magnetic susceptibilities along the crystal axes of Y-124 which are ordered $\chi_{c}>\chi_{a}>\chi_{b}$. The technique is explained in their paper as follows:

"If a magnetic field is initially applied to a suspension of single crystal grains they will rotate so the $c$-axis is aligned with the field direction with a characteristic grain rotation time which is determined by the field strength, susceptibility anisotropy, and the viscosity of the suspending fluid. If the field is then switched to a second direction orthogonal to the first for a time which is short compared to the grain rotation time the crystal axis with in- 
termediate susceptibility, the $a$-axis, will rotate towards the field direction. Repetition of this cycle of field switching leads to biaxial alignment of the grains." [106]

A schematic diagram of the biaxial alignment rig is shown in Fig. 3.2. The rig is homemade, comprising a rotating platform that is driven by a chart recorder. The chart recorder is controlled by a square wave input from a function generator. The sample orientation is switched between 0 and 90 degrees relative to the direction of the magnetic field with dwell times dependent on the material being aligned. For Y-124 dwell times of two seconds in the primary position and one second in the secondary position were found to be appropriate. Sample alignment can be confirmed by the presence and absence of specific peaks in X-ray diffraction spectra, and the quality of the alignment can be determined via analysis of rocking curves.

The ${ }^{16} \mathrm{O} /{ }^{18} \mathrm{O}$ substituted pellets were cut in half and ground separately in isopropanol for 30 minutes by hand. The small quantities of powder $(<1 \mathrm{~g})$ precluded the use of a ball mill. The powders were aligned in Struers EpoFix epoxy. Epofix is a low viscosity epoxy that takes approximately 12 hours to cure at room temperature. The resin and powder were combined in a ratio of 1.28:1 (resin:powder) by weight and mixed using a mechanical milkshake mixer with a high shear rate $(\approx 4000 \mathrm{RPM})$ for two minutes. The hardener was mixed in by hand and the mixture was immediately poured into a flexible teflon mould with internal dimensions $7 \mathrm{~mm} \times 7 \mathrm{~mm} \times 5 \mathrm{~mm}$. The mould was then placed on the biaxial alignment rig under an applied field of one Tesla where it was left to set overnight. 


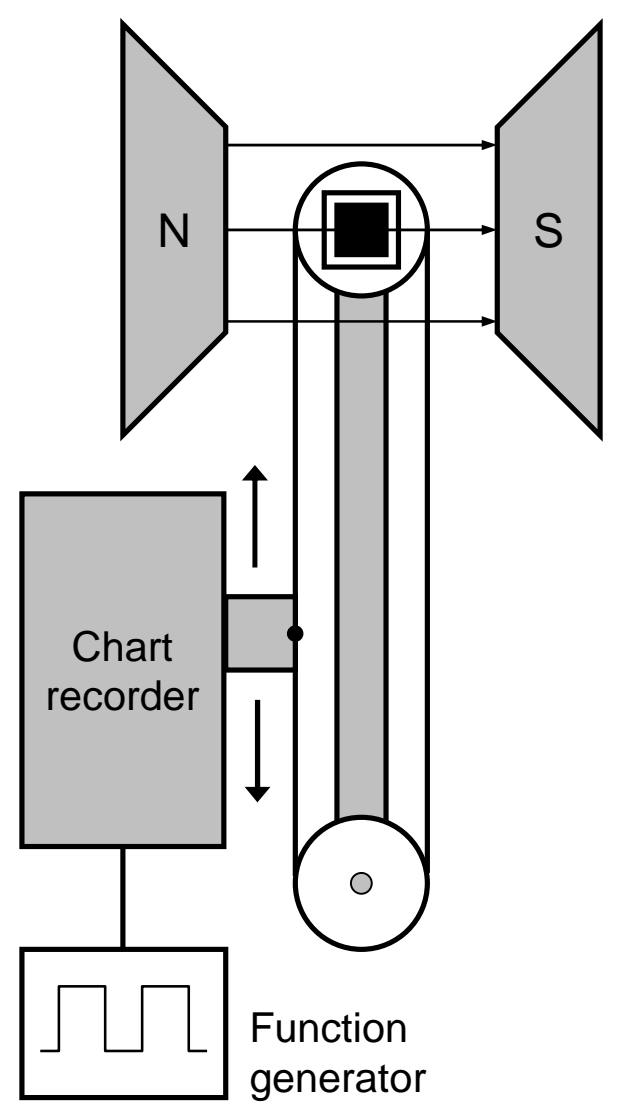

Figure 3.2: Schematic diagram of the biaxial alignment rig. The chart recorder switches the orientation of the sample by 90 degrees relative to the magnetic field. 


\subsection{Measurements}

Masses were measured with a Mettler AE200 electronic analytical balance with $0.1 \mathrm{mg}$ readability and $205 \mathrm{~g}$ capacity.

X-ray diffraction measurements were performed on Phillips PW1700 and PW3700 series Bragg-Brentano diffractometers with fixed divergence slits and graphite diffracted beam monochromators. The radiation used was cobalt $K_{\alpha}$ and the detectors were xenon filled proportional counters.

Raman spectra were obtained at room temperature using a Jobin Yvon LabRam confocal spectrometer with a $633 \mathrm{~nm} 3 \mathrm{~mW}$ laser, a 600 lines $/ \mathrm{mm}$ holographic grating and a liquid nitrogen cooled CCD detector.

A Shimadzu SALD-2001 laser diffraction particle size analyser was used to measure the size distributions of portions of the powders used in manufacturing the biaxially aligned samples. The particles were suspended in anhydrous isopropyl alcohol and utrasonicated for six minutes. The size distributions of the grains in the composite powder-epoxy samples were determined from images taken with a $L E O 400$ scanning electron microscope. The images were analysed with ImageJ, an image processing and analysis software program (available from http://rsb.info.nih.gov/ij/).

Magnetization and AC susceptibility measurements were performed on a Quantum Design MPMS XL SQUID magnetometer. Bulk pellet samples were mounted in plastic straws. Powder samples were sealed in gelatin capsules which were then mounted inside plastic straws. The composite powderepoxy samples were mounted on a horizontal sample rotator with a small amount of $G E$ varnish. The horizontal rotator allows samples to be rotated 
about the horizontal axis and was employed to optimize the alignment between the crystallographic axes and the magnetic field. 


\section{Chapter 4}

\section{Experimental Results}

\subsection{Sample characterization}

The X-ray diffraction spectrum taken from the surface of a Y-124 pellet after the final cook in 60 bars $\mathrm{O}_{2}$ at $950^{\circ} \mathrm{C}$ is shown in Fig. 4.1. There is a good match with the powder diffraction database pattern (powder diffraction file \#43-0402) for $\mathrm{YBa}_{2} \mathrm{Cu}_{4} \mathrm{O}_{8}$, and the spectrum is free of any significant impurity phases. Note that the quality of the bulk is expected to be superior to that of the surface.

A pellet $12 \mathrm{~mm}$ in diameter and approximately $3 \mathrm{~mm}$ thick was cut in half and annealed ten times over a six day period in the isotope exchange rig. Based on the sample mass and Eqn. 3.1 it was estimated that ten steps would result in a $94.4 \%$ exchange. The sample masses before and after the exchange are given in Table 4.1. The mass of the ${ }^{16} \mathrm{O}$ sample is virtually unchanged while the change in mass of the ${ }^{18} \mathrm{O}$ sample is consistent with a $93.4 \%$ exchange. 


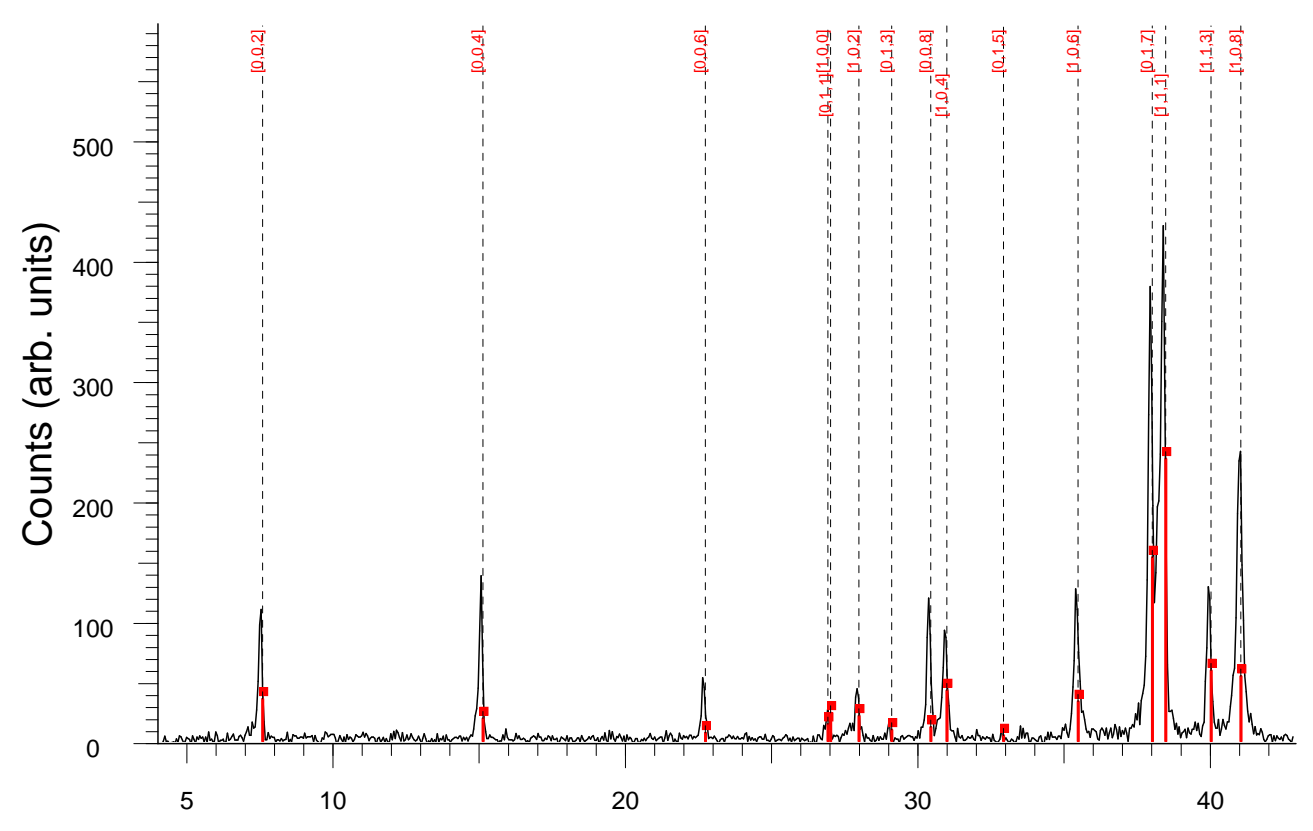

$2 \theta$

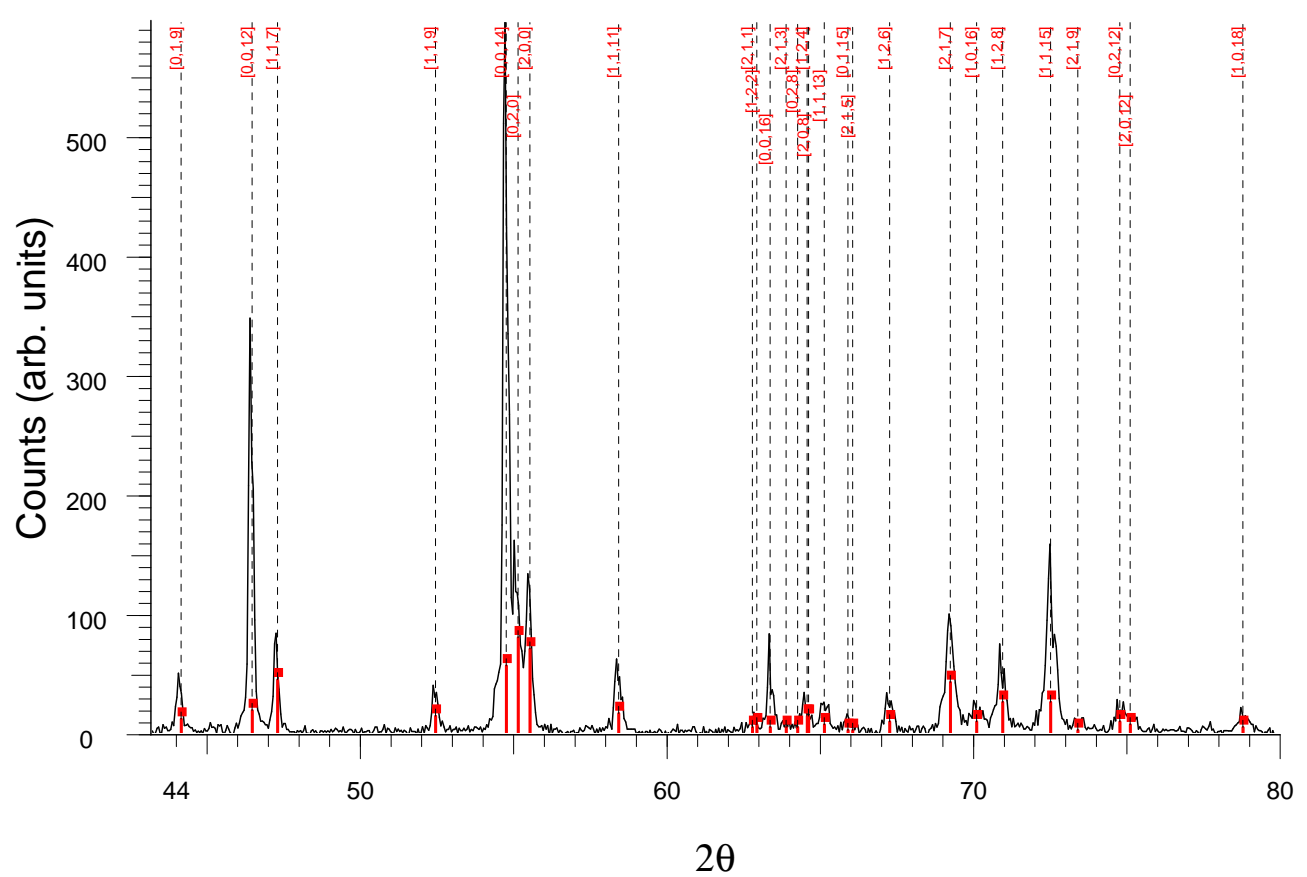

Figure 4.1: X-ray diffraction spectrum obtained from the surface of a sample pellet after the final cook at $950^{\circ} \mathrm{C}$. The expected pattern for $\mathrm{YBa}_{2} \mathrm{Cu}_{4} \mathrm{O}_{8}$ is shown in red along with the Miller indices. 
Table 4.1: Sample masses before and after oxygen isotope exchange.

\begin{tabular}{|c|c|c|c|}
\hline Sample & Initial & Final & \% Exchanged \\
\hline${ }^{16} \mathrm{O}$ & $0.8122 \mathrm{~g}$ & $0.8120 \mathrm{~g}$ & - \\
${ }^{18} \mathrm{O}$ & $0.9460 \mathrm{~g}$ & $0.9650 \mathrm{~g}$ & 93.4 \\
\hline
\end{tabular}

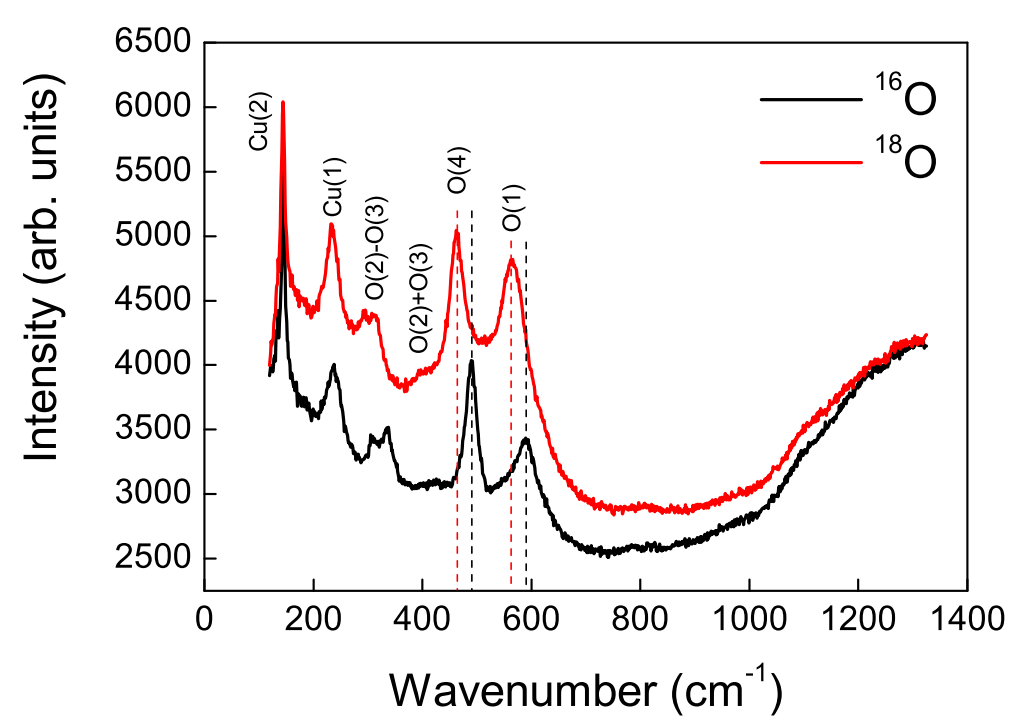

Figure 4.2: Raman spectra taken from the surface of the ${ }^{16} \mathrm{O} /{ }^{18} \mathrm{O}$ substituted Y-124 sample pellets. Phonon mode assignments are from Refs. [108, 107]

The isotope exchange was confirmed in Raman spectra taken from the sample surfaces, plotted in Fig. 4.2. As expected the oxygen modes are shifted to lower frequencies in the ${ }^{18} \mathrm{O}$ substituted sample and there is no discernable shift in the copper modes. The measured shifts in the $\mathrm{O}(1)$ and $\mathrm{O}$ (4) lines, listed in Table 4.2, compare well with the theoretical shifts from Ref. [107]. In a first order approximation the fractional change in frequency is given by $1-\sqrt{16 / 18}$. For the $\mathrm{O}(4)$ mode this corresponds to a shift of $28 \mathrm{~cm}^{-1}$ which matches the observed shift.

${ }^{16} \mathrm{O}$ and ${ }^{18} \mathrm{O}$ substituted biaxially-aligned composite powder-epoxy sam- 
Table 4.2: Oxygen isotope induced Raman shift, $\Delta$, in the $\mathrm{O}(4)$ and $\mathrm{O}(1)$ phonon modes. Values from Ref. [107] are included for comparison.

\begin{tabular}{|c|c|c|c|c|}
\hline Peak & ${ }^{16} \mathrm{O} \mathrm{cm}^{-1}$ & ${ }^{18} \mathrm{O} \mathrm{cm}^{-1}$ & $\Delta \mathrm{cm}^{-1}$ & $\Delta$ Ref. [107] $\mathrm{cm}^{-1}$ \\
\hline $\mathrm{O}(4)$ & 490.1 & 462.0 & 28.1 & 28.2 \\
$\mathrm{O}(1)$ & 589.4 & 561.7 & 27.7 & 26.3 \\
\hline
\end{tabular}

ples were prepared following the procedure outlined in section 3.3. X-ray diffraction spectra taken from three orthogonal faces of the samples are shown in Fig. 4.3. The spectra measured from the faces normal to the $c$-axis (shown in black) consist of the periodic reflections from the $a b$-planes with Miller indices given by $[0,0,2 n]$. There is a small reflection from the $[1,0,8]$ plane. The spectra taken from faces normal to the $a$ - and $b$-axes are dominated by the $[2,0,0]$ and $[0,2,0]$ reflections respectively.

A close-up of the orthogonal $[0,0,14],[0,2,0]$ and $[2,0,0]$ peaks is shown in Fig. 4.4. The observation of only a single peak in each spectrum indicates good biaxial texture. Rocking curves were measured on each of these peaks. The full width at half maximum (FWHM) values of the rocking curves are listed in Table 4.3. The results indicate that the quality of alignment is comparable between the two samples in the $a$ and $c$ directions. The degree of alignment and agreement between samples is worst in the $b$-direction, with the FWHM of the ${ }^{18} \mathrm{O}$ sample some four degrees larger than the ${ }^{16} \mathrm{O}$ sample. This is not surprising as the $b$-axis lies transverse to the magnetic field during the alignment process.

Using a diamond wheel saw, rods were cut from the edges of the samples along the three orthogonal directions for the AC susceptibility measurements. The dimensions were roughly $1.5 \times 1.5 \times 7 \mathrm{~mm}$ for the $a$ - and $c$-axis oriented 


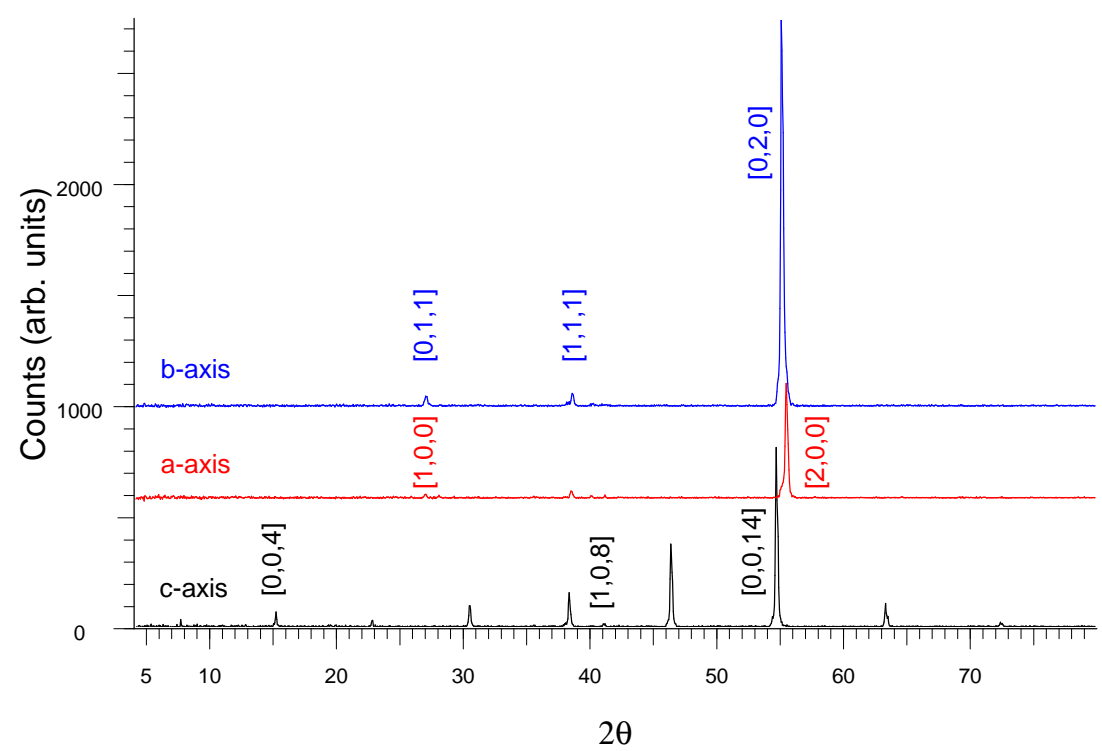

(a) ${ }^{16} \mathrm{O}$

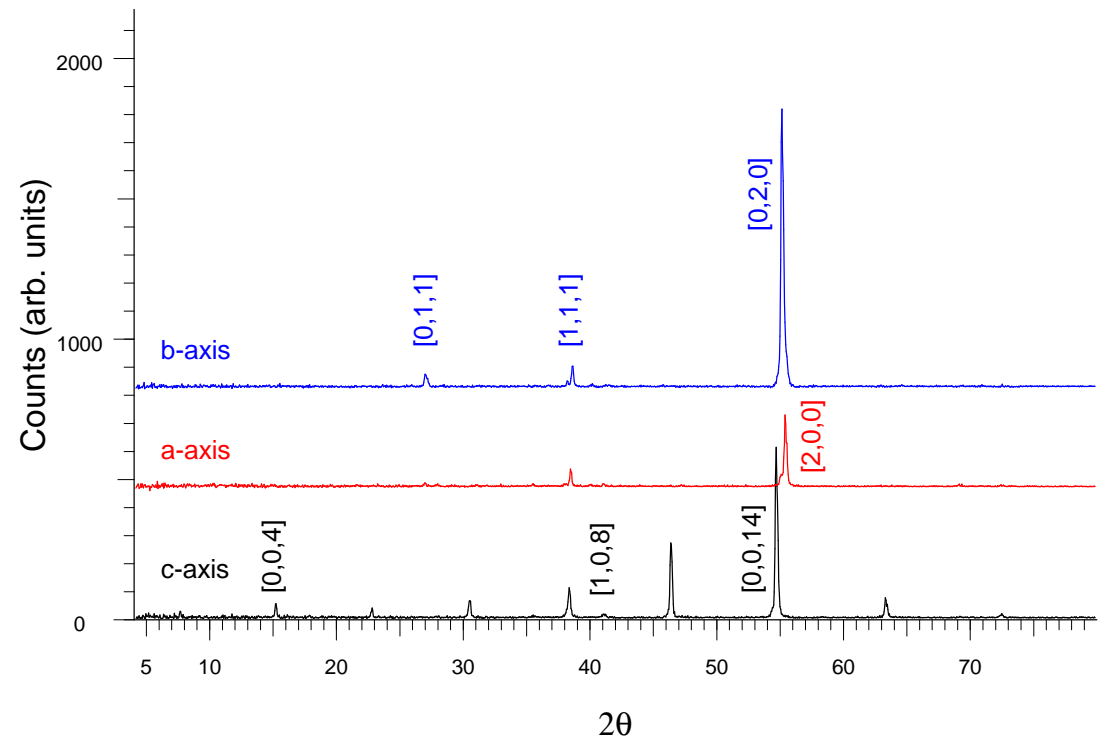

(b) ${ }^{18} \mathrm{O}$

Figure 4.3: X-ray diffraction spectra taken from three orthogonal faces of the biaxially aligned samples. The spectra measured from faces orthogonal to the $a$ and $b$-axes have been shifted vertically for clarity. 


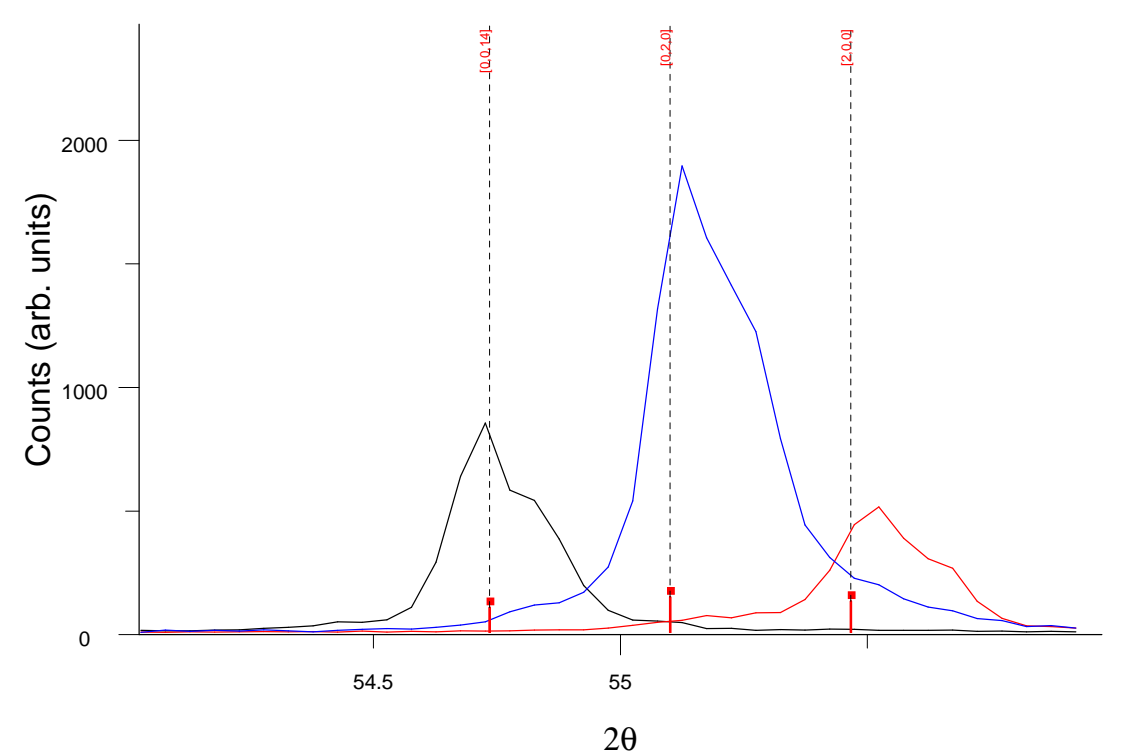

(a) ${ }^{16} \mathrm{O}$

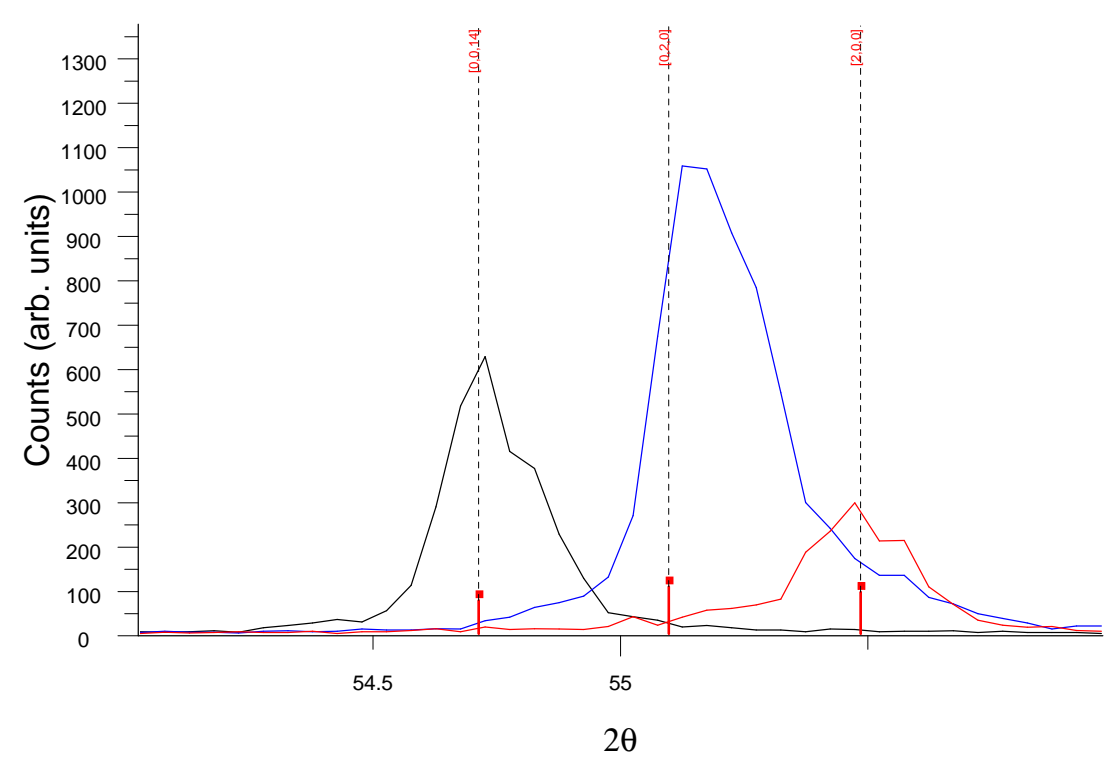

(b) ${ }^{18} \mathrm{O}$

Figure 4.4: Close up of the $[0,0,14],[0,2,0]$ and $[2,0,0]$ Bragg peaks as measured from three orthogonal faces of the biaxially aligned samples. 
Table 4.3: Rocking curve full width half maximum values of the orthogonal $[2,0,0]$, $[0,2,0]$ and $[0,0,14] \mathrm{X}$-ray diffraction peaks.

\begin{tabular}{|c|c|c|c|}
\hline Sample & {$[2,0,0]$} & {$[0,2,0]$} & {$[0,0,14]$} \\
\hline${ }^{16} \mathrm{O}$ & $5.1^{\circ}$ & $5.8^{\circ}$ & $4.3^{\circ}$ \\
${ }^{18} \mathrm{O}$ & $5.5^{\circ}$ & $10^{\circ}$ & $5.2^{\circ}$ \\
\hline
\end{tabular}

Table 4.4: Mass, volume and density parameters of the AC susceptibility samples. Subscripts $c, e$ and $p$ stand for composite, epoxy and powder respectively. The right-most column lists the powder volume fraction.

\begin{tabular}{|c|c|c|c|c|c|c|c|}
\hline Sample & $\begin{array}{c}m_{c} \\
(\mathrm{mg})\end{array}$ & $\begin{array}{c}V_{c} \\
\left(10^{-3} \mathrm{~cm}^{3}\right)\end{array}$ & $\begin{array}{c}\rho_{c} \\
\left(\mathrm{gcm}^{-3}\right)\end{array}$ & $\begin{array}{c}\rho_{e} \\
\left(\mathrm{gcm}^{-3}\right)\end{array}$ & $\begin{array}{c}\rho_{p} \\
\left(\mathrm{gcm}^{-3}\right)\end{array}$ & $\begin{array}{c}V_{p} \\
\left(10^{-3} \mathrm{~cm}^{3}\right)\end{array}$ & $\begin{array}{c}\text { Vol. frac. } \\
(\%)\end{array}$ \\
\hline${ }^{16} \mathrm{O}_{a}$ & 26.9 & 16.5 & 1.63 & 1.10 & 6.12 & 1.74 & 10.5 \\
${ }^{16} \mathrm{O}_{b}$ & 18.4 & 11.6 & 1.59 & 1.10 & 6.12 & 1.13 & 9.73 \\
${ }^{16} \mathrm{O}_{c}$ & 27.0 & 16.3 & 1.66 & 1.10 & 6.12 & 1.82 & 11.2 \\
${ }^{18} \mathrm{O}_{a}$ & 24.2 & 14.8 & 1.64 & 1.10 & 6.25 & 1.55 & 10.5 \\
${ }^{18} \mathrm{O}_{b}$ & 17.6 & 10.8 & 1.63 & 1.10 & 6.25 & 1.11 & 10.3 \\
${ }^{18} \mathrm{O}_{c}$ & 27.2 & 16.4 & 1.66 & 1.10 & 6.25 & 1.78 & 10.9 \\
\hline
\end{tabular}

rods and $1.5 \times 1.5 \times 5 \mathrm{~mm}$ for the $b$-axis oriented rods. The volume of powder, $V_{p}$, in the composite powder-epoxy samples can be calculated from the following expression

$$
V_{p}=V_{c}\left(\frac{\rho_{e}-\rho_{c}}{\rho_{e}-\rho_{p}}\right)
$$

where $V_{c}$ is the volume of the composite sample and $\rho_{e}, \rho_{c}$ and $\rho_{p}$ are the densities of the epoxy, composite, and powder respectively. Table 4.4 lists the volume fraction of Y-124 powder in each of the samples calculated from Eqn. 4.1.

The size distributions of samples of ${ }^{16} \mathrm{O} /{ }^{18} \mathrm{O}$ Y-124 loose powder are shown in Fig. 4.5. Particles in the ${ }^{16} \mathrm{O}$ powder were found to have a mean diameter of $4.1 \mu \mathrm{m}$ and a standard deviation of $0.34 \mu \mathrm{m}$. In comparison, particles in the ${ }^{18} \mathrm{O}$ powder were found to be larger but more narrowly distributed, 
with mean diameter of $6.7 \mu \mathrm{m}$ and standard deviation of $0.3 \mu \mathrm{m}$.

The size distributions of the grains in the composite powder-epoxy samples were determined from scanning electron microscope (SEM) images. The SEM technique requires conductive samples. The images were taken from polished carbon-coated surfaces of pieces of the samples that were left over following the removal of the rod shaped pieces mentioned earlier. Some representative SEM images are shown in Fig. 4.6. The grains appear to be evenly distributed throughout the images. The size distributions as determined from multiple images are plotted in Fig. 4.7.

Comparison of these distributions with those of the loose powders shows that in the process of mixing the powder and resin the particles (or possibly clumps) have been broken down even further. The distributions from the SEM images are more evenly matched although, as seen in the inset to Fig. 4.7, the particles in the ${ }^{18} \mathrm{O}$ sample are still slightly larger on average.

\subsection{Magnetization}

Figure 4.8 shows the DC mass magnetization of the ${ }^{16} \mathrm{O}$ and ${ }^{18} \mathrm{O}$ substituted $\mathrm{YBa}_{2} \mathrm{Cu}_{4} \mathrm{O}_{8}$ bulk pellet samples under an applied field of $100 \mathrm{Oe}$. The transition temperatures of the ${ }^{16} \mathrm{O}$ and ${ }^{18} \mathrm{O}$ samples are $81.96 \mathrm{~K}$ and $81.49 \mathrm{~K}$ respectively. The isotope effect in $T_{c}$ is therefore 0.046 . This compares well with the results of Khasanov et al. who obtained a value of 0.048(8)[103]. 


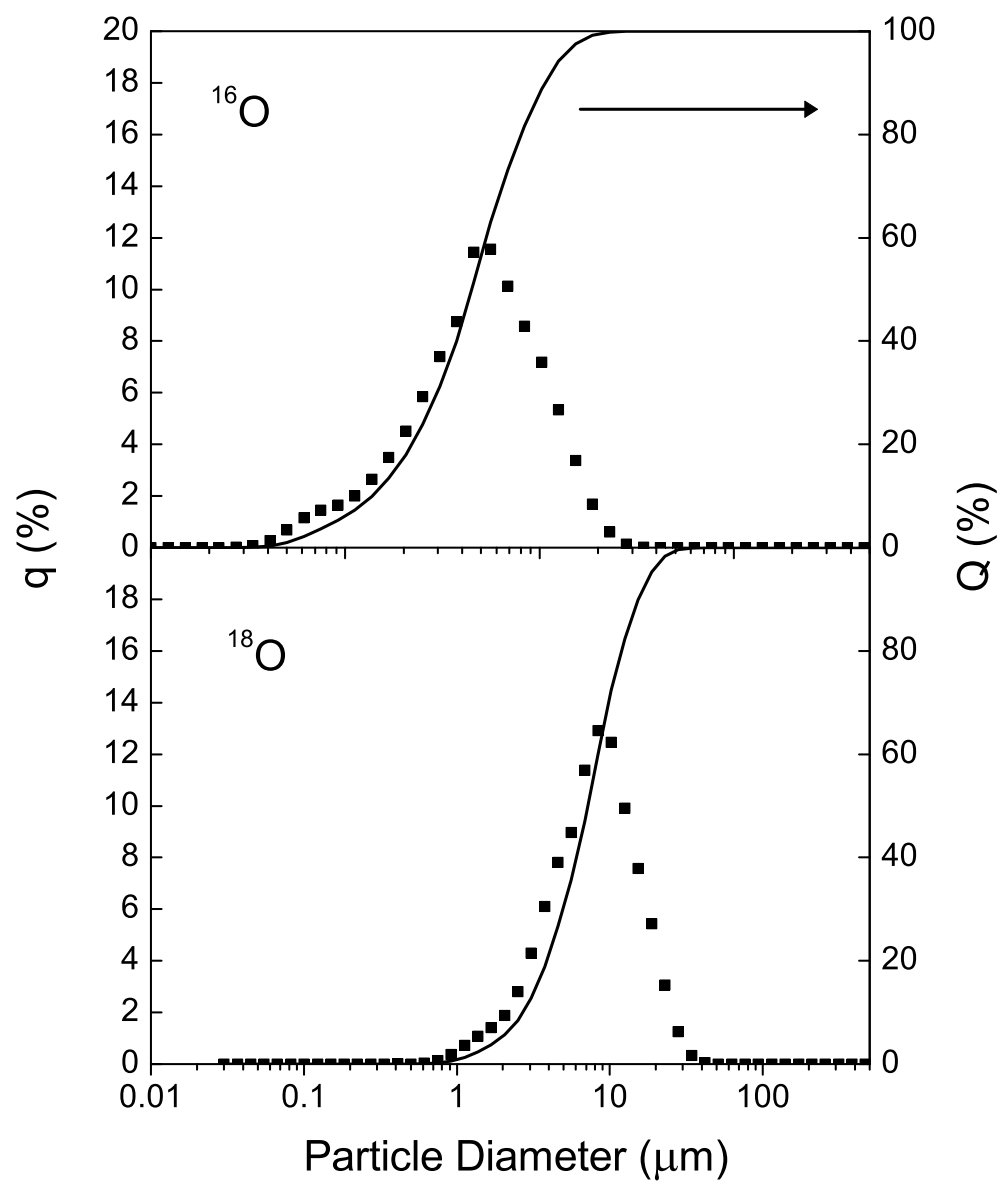

Figure 4.5: Differential (q) and cumulative (Q) particle size distributions of ${ }^{16} \mathrm{O}$ and ${ }^{18} \mathrm{O}$ substituted $\mathrm{Y}-124$ loose powders, as determined by laser diffraction measurements. 


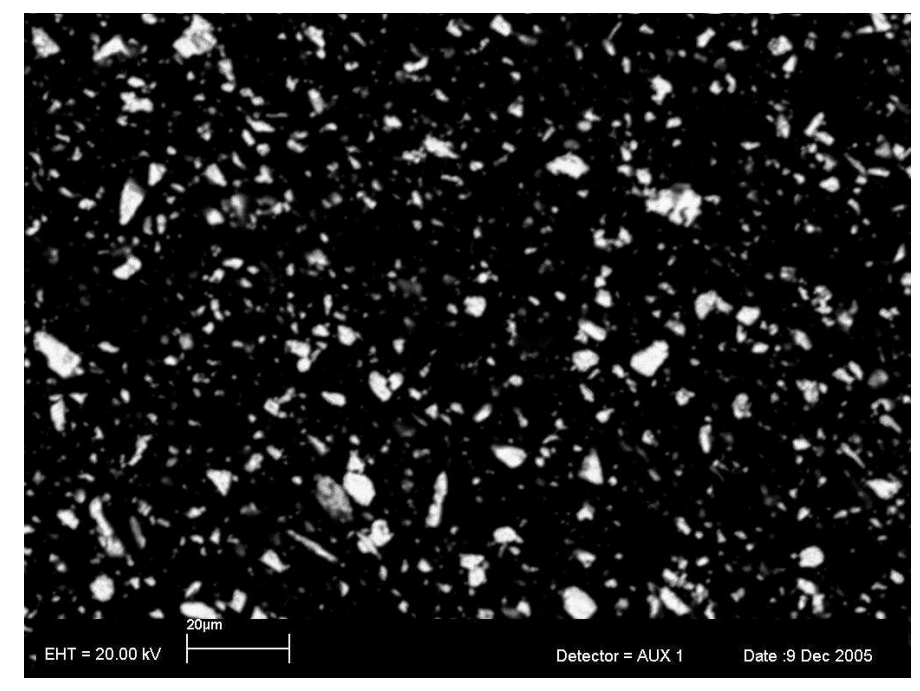

(a) ${ }^{16} \mathrm{O}$

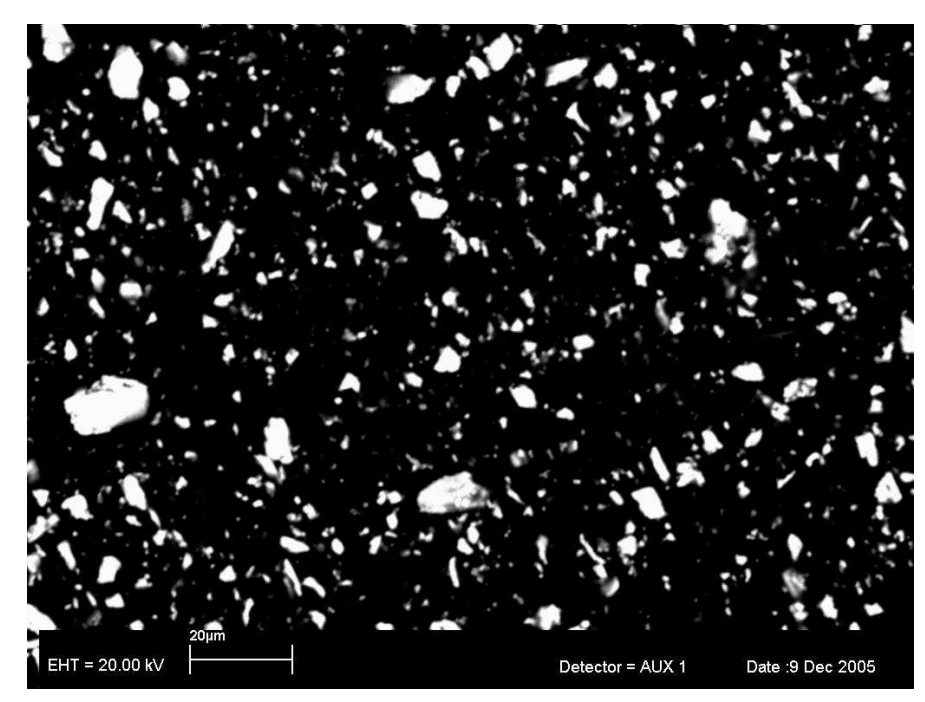

(b) ${ }^{18} \mathrm{O}$

Figure 4.6: Scanning electron microscope images showing the distribution of grains in the ${ }^{16} \mathrm{O}$ and ${ }^{18} \mathrm{O}$ substituted powder-epoxy composite samples. The scale is $20 \mu \mathrm{m}$. 


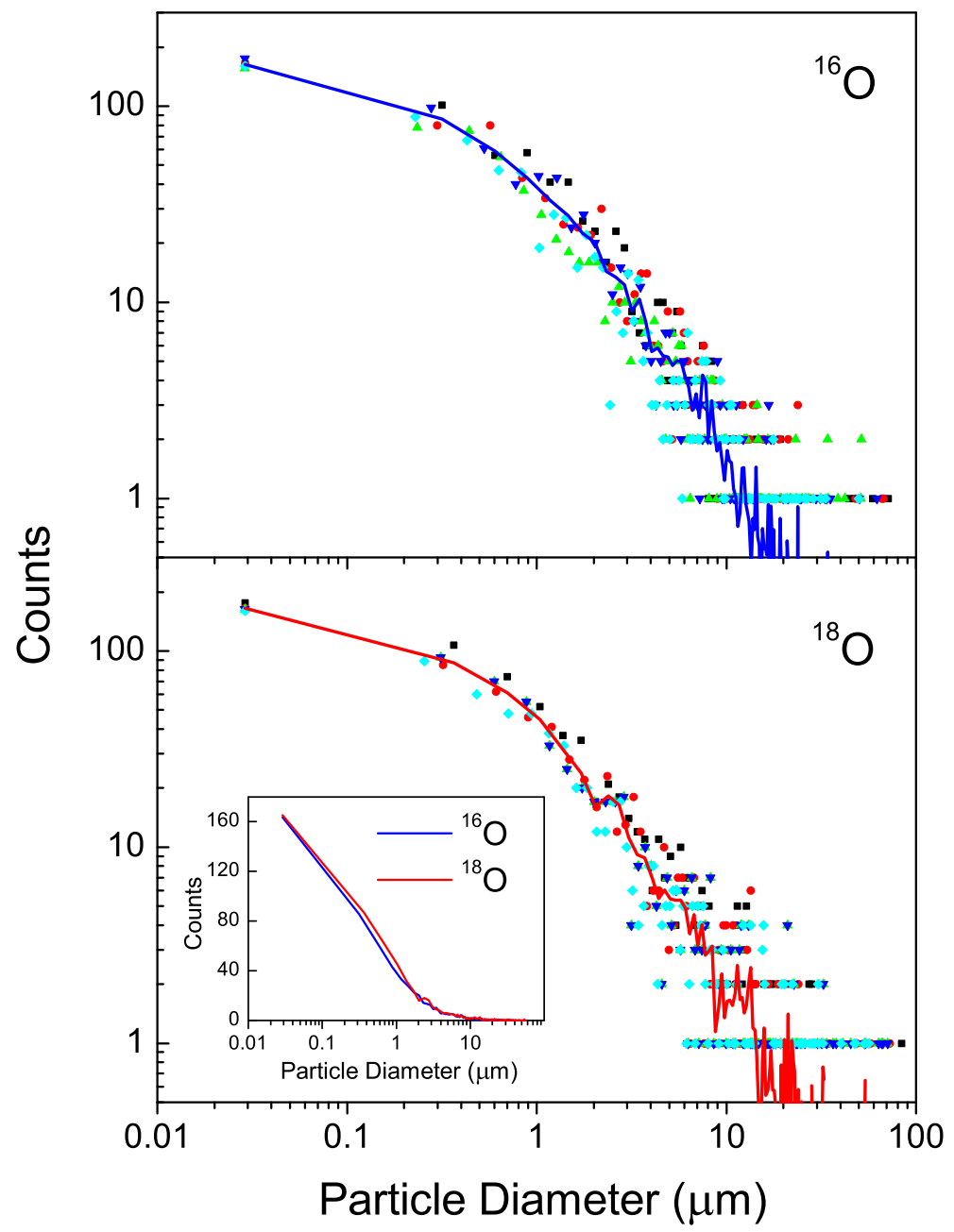

Figure 4.7: Distribution of particle sizes in ${ }^{16} \mathrm{O}$ and ${ }^{18} \mathrm{O}$ substituted $\mathrm{Y}-124$ biaxially aligned samples as determined from SEM images. The inset compares the averages of the ${ }^{16} \mathrm{O}$ and ${ }^{18} \mathrm{O}$ datasets. 


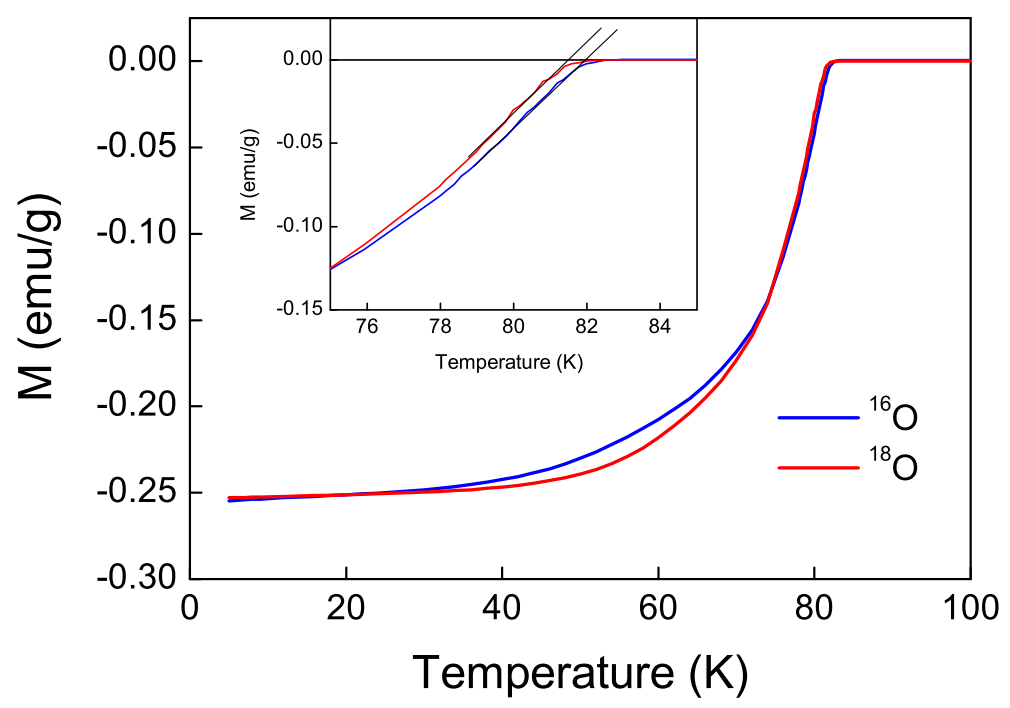

Figure 4.8: DC mass magnetization of ${ }^{16} \mathrm{O}$ and ${ }^{18} \mathrm{O}$ substituted $\mathrm{YBa}_{2} \mathrm{Cu}_{4} \mathrm{O}_{8}$ bulk pellet samples under an applied field of 100Oe. The inset shows the magnetization near $T_{c}$.

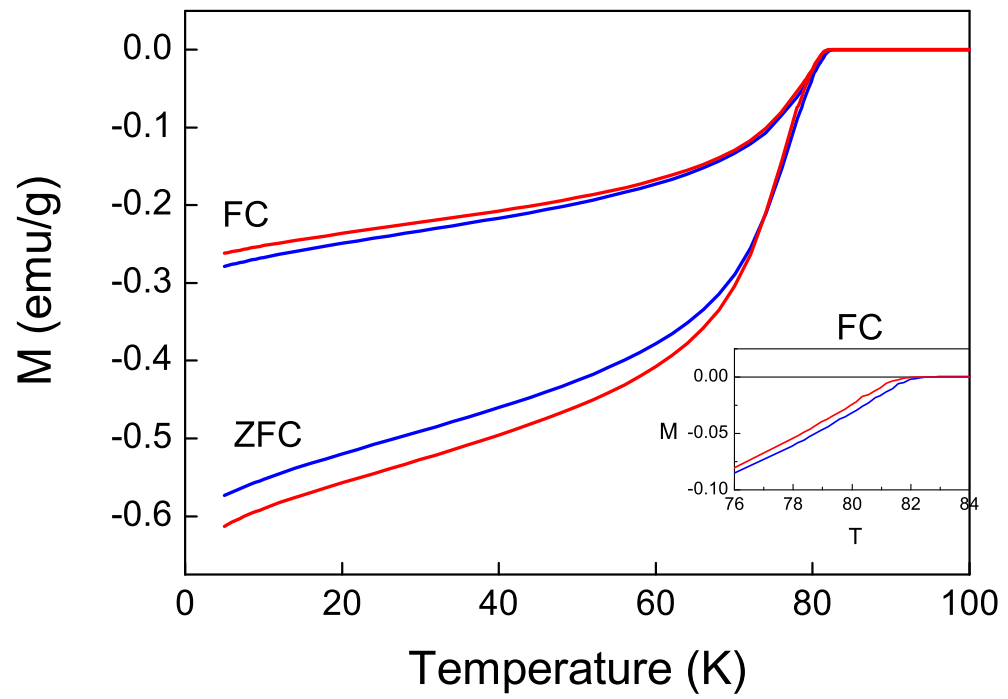

Figure 4.9: Field-cooled and zero-field-cooled DC mass magnetization of ${ }^{16} \mathrm{O}$ and ${ }^{18} \mathrm{O}$ substituted $\mathrm{YBa}_{2} \mathrm{Cu}_{4} \mathrm{O}_{8}$ powder samples under an applied field of $100 \mathrm{Oe}$. The inset shows the field-cooled magnetization near $T_{c}$. 


\subsection{AC Susceptibility}

AC susceptibility measurements were performed on the rod-shaped composite samples with the field aligned parallel to the $a-, b$ - and $c$-axes, alternately. A rotatable sample holder was used to obtain an optimal sample alignment. Temperature-dependent measurements were performed on warming under an AC field of amplitude 1 Oe and frequency $332.45 \mathrm{~Hz}$. The susceptibility at $5 \mathrm{~K}$ was found to be independent of field for amplitudes up to $5 \mathrm{O}$ e and frequencies up to $699.63 \mathrm{~Hz}$ indicating good grain separation and the absence of weak links.

The temperature dependent AC susceptibility is shown in Fig. 4.10 for all six combinations of isotope and alignment. The susceptibility in S.I. units is dimensionless and was calculated using the following relation

$$
\chi(T)=\frac{m^{\prime}(T)}{V H} \times 4 \pi
$$

where $m^{\prime}$ is the measured signal in emu, $H$ is the applied field amplitude in Oe and $V$ is the volume of powder in $\mathrm{cm}^{3}$. For a perfectly diamagnetic infinitely long thin wire parallel to the applied field $\chi=-1$.

\subsection{Penetration Depth \& Superfluid Density}

If a perfectly diamagnetic object is subjected to a uniform applied field $H_{0}$ the field at the surface of the object is renormalized to a value $H$ given by[44]

$$
H=\frac{1}{1-n} H_{0}
$$




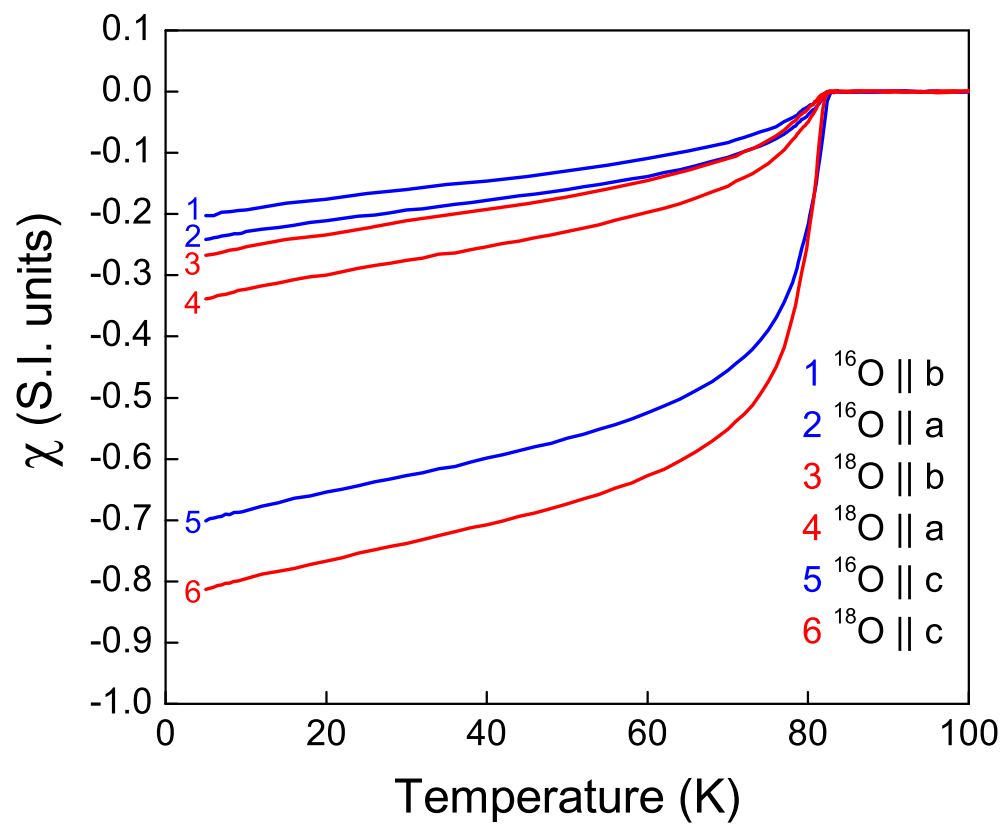

Figure 4.10: $\mathrm{AC}$ volume susceptibility of the biaxially aligned ${ }^{16} \mathrm{O}$ and ${ }^{18} \mathrm{O}$ substituted samples for fields parallel to the $a, b$ and $c$ axes. 


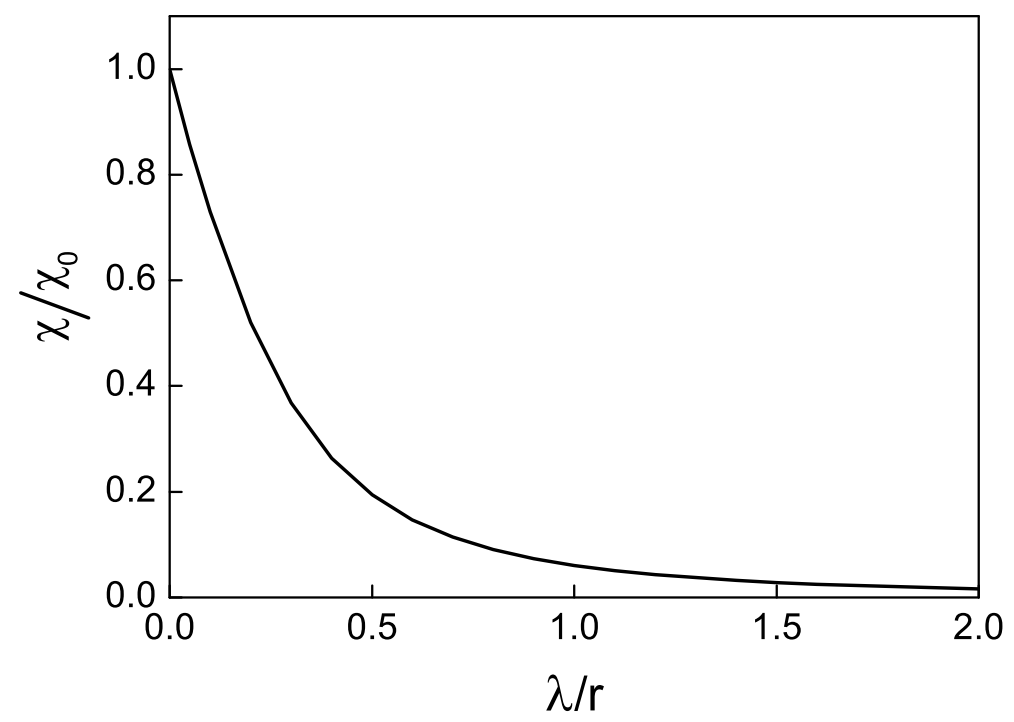

Figure 4.11: Suppression of the normalised susceptibility as a function of $\lambda / r$ as given by Eqn. 4.4.

$n$ is the demagnetization factor and its value depends on the geometry of the sample. For a sphere $n=\frac{1}{3}$. Therefore the susceptibility of a perfectly diamagnetic spherical grain in S.I. units is $\chi_{0}=-1.5$. For a superconducting sphere of radius $r$ with magnetic penetration depth $\lambda$ the normalised susceptibility is given by the so-called "Shoenberg formula" $[109,110]$

$$
\frac{\chi}{\chi_{0}}=1-\frac{3 \lambda}{r} \operatorname{coth}\left(\frac{r}{\lambda}\right)+\frac{3 \lambda^{2}}{r^{2}}
$$

Equation 4.4 describes the suppression in susceptibility that arises due to penetration depth effects and is plotted as a function of $\lambda / r$ in Fig. 4.11. For a distribution of spherical grains the effective normalised susceptibility 
Table 4.5: Susceptibilies $(\chi)$, normalised susceptibilities $\left(\chi / \chi_{0}\right)$, and $a b$-plane penetration depths $\left(\lambda_{a b}\right)$ as calculated from the size distributions determined by SEM image analysis.

\begin{tabular}{|c|c|c|c|}
\hline Sample & $\chi$ & $\chi / \chi_{0}$ & $\lambda_{a b}(\mathrm{~nm})$ \\
\hline${ }^{16} \mathrm{O}$ & -0.702 & -0.468 & 2961 \\
${ }^{18} \mathrm{O}$ & -0.813 & -0.542 & 2964 \\
\hline
\end{tabular}

is given by[110]

$$
\left(\frac{\chi}{\chi_{0}}\right)_{e f f}=\frac{\int\left(1-\frac{3 \lambda}{r} \operatorname{coth}\left(\frac{r}{\lambda}\right)+\frac{3 \lambda^{2}}{r^{2}}\right) r^{3} g(r) d r}{\int r^{3} g(r) d r}
$$

where $g(r)$ is the measured grain size distribution.

Another potential source of susceptibility reduction comes from the renormalized field from one particle perturbing the fields experienced by neighbouring particles. An investigation by Campbell et al.[111] shows that this effect becomes significant for superconductor volume fractions above $20 \%$ and so this effect will be neglected in the following analysis.

The $a b$-plane penetration depths, $\lambda_{a b}$, at $5 \mathrm{~K}$ were calculated from Eqn. 4.5 using the grain size distributions determined from SEM image analysis. The results are shown in Table 4.5. The values obtained are on the order of $3 \mu \mathrm{m}$, far in excess of the $127 \mathrm{~nm}$ measured by Panagopoulos et al. on $c$ axis grain-aligned samples[112]. In an effort to locate the source of this discrepancy $\lambda_{a b}$ was calculated as a function of the upper cut-off radius used in the integrations of Eqn. 4.5. The results are plotted in Fig. 4.12. Also plotted is the percentage of particles that are larger than the upper cut-off radius. The plot clearly illustrates the enormous effect that a small number of large grains in the distribution has on the calculated values of $\lambda_{a b}$. 


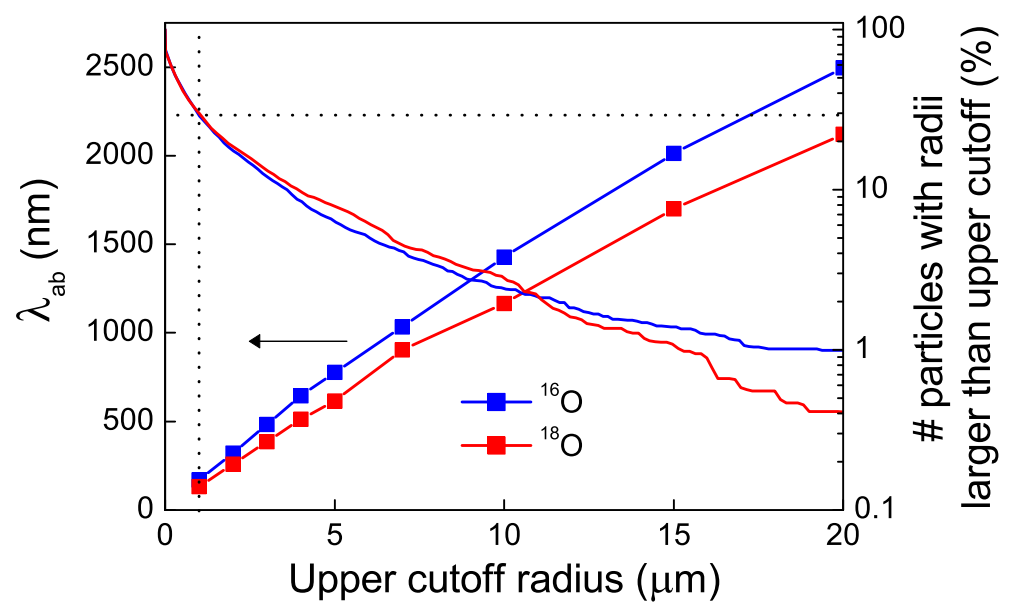

Figure 4.12: Variation of the $T=5 \mathrm{~K} a b$-plane penetration depth (calculated from corrected ACS data) with grain distribution upper cut-off radius. Also plotted is the percentage of particles with radii larger than the upper cut-off radius.

Guided by the fact that the mean particle size was found to be lower in the composite samples relative to the free powders we presume that the high shear rate in the mixing with resin broke up aggregates. Certainly no more milling was employed so the high end of the distribution is almost certainly affected, probably dominated, by aggregates and polycrystalline grains. These will of course yield a susceptibility reflecting the individual grain sizes, not their aggregate size. Accordingly, our strategy for analysis was as follows. Firstly, an upper cut-off radius $(=0.76 \mu \mathrm{m})$ for the ${ }^{16} \mathrm{O}$ distribution was chosen so that the calculated value of $\lambda_{a b}$ reproduced the result obtained by Panagopoulos et al.[112]. (Note that this value is also confirmed by optical measurements[113]). Secondly, the oxygen isotope coefficient in $\lambda_{a b}(=0.18 \pm 0.06)$, as determined by muon spin relaxation measurements on Y-124 by Khasanov et al. [103], was used to deduce a value of $\lambda_{a b}$ for the ${ }^{18} \mathrm{O}$ 
Table 4.6: $a b-, b c$ - and $a c$-plane penetration depths $(\lambda)$ at $5 \mathrm{~K}$, as calculated from the particle size distributions determined from SEM image analysis with fixed upper cut-off radii. $\chi / \chi_{0}$ is the normalised AC susceptibility and $\alpha(\lambda)$ is the oxygen isotope effect exponent in the penetration depth.

\begin{tabular}{|c|c|c|c|c|c|}
\hline & $\chi / \chi_{0,{ }^{16} O}$ & $\chi / \chi_{0,{ }^{18} O}$ & $\lambda_{{ }_{16} O}(\mathrm{~nm})$ & $\lambda_{{ }^{18} O}(\mathrm{~nm})$ & $\alpha(\lambda)$ \\
\hline$a b$ & -0.468 & -0.542 & 131 & $134(1)$ & $-0.18(6)$ \\
$b c$ & -0.161 & -0.225 & 337 & $335(3)$ & $+0.05(7)$ \\
$a c$ & -0.135 & -0.178 & 377 & $396(4)$ & $-0.40(7)$ \\
\hline
\end{tabular}

Table 4.7: $a$-, $b$ - and $c$-axis penetration depths $(\lambda)$ at $5 \mathrm{~K}$ and corresponding isotope exponents $(\alpha(\lambda))$ as calculated from the $a b-, b c$ - and $a c$-plane penetration depths.

\begin{tabular}{|c|c|c|c|c|}
\hline & $\lambda_{16}(\mathrm{~nm})$ & $\lambda_{18} O(\mathrm{~nm})$ & $\alpha(\lambda)$ & $\lambda(\mathrm{nm})$ Ref. $[112]$ \\
\hline$a$ & 147 & $158 \pm 2$ & $-0.6 \pm 0.1$ & 154 \\
$b$ & 117 & $113 \pm 1$ & $0.27 \pm 0.07$ & 86 \\
$c$ & 970 & $990 \pm 10$ & $-0.16 \pm 0.08$ & $615 \pm 90$ \\
\hline
\end{tabular}

substituted sample. This, in turn, implied a cut-off radius $=0.96 \pm 0.01 \mu \mathrm{m}$ for the ${ }^{18} \mathrm{O}$ distribution. This is consistent with our observation of a higher mean radius for the ${ }^{18} \mathrm{O}$ sample. Finally, the $b c$ - and $a c$-plane penetration depths were calculated assuming these cut-off radii. The results are listed in Table 4.6.

Assuming the relation $\lambda_{x y}^{2}=\lambda_{x} \lambda_{y}$, the $a-, b$ - and $c$-axis penetration depths and corresponding isotope effect coefficients were calculated. They are listed in Table 4.7 along with values determined by Panagopoulos et al.[112]. Although the match isn't exact the relative magnitudes are comparable. We find a smaller anisotropy than has been reported from AC susceptibility[112] and infrared measurements[113] and this reflects the supposed aggregation which will exhibit a more averaged susceptibility.

The superfluid density, $\rho_{s}$, is proportional to $1 / \lambda^{2}$. The plane and axis 
Table 4.8: Plane and axis superfluid densities $\left(\rho_{s}\right)$ at $5 \mathrm{~K}$ and isotope effect coefficients $\left(\alpha\left(\rho_{s}\right)\right)$.

\begin{tabular}{|c|c|c|c|}
\hline & $\rho_{s,{ }^{16} \mathrm{O}}\left(\mu \mathrm{m}^{-2}\right)$ & $\rho_{s,{ }^{18} \mathrm{O}}\left(\mu \mathrm{m}^{-2}\right)$ & $\alpha\left(\rho_{s}\right)$ \\
\hline$a b$ & 58.3 & $55.7 \pm 0.8$ & $0.4 \pm 0.1$ \\
$b c$ & 8.8 & $8.9 \pm 0.2$ & $-0.09 \pm 0.20$ \\
$a c$ & 7.0 & $6.4 \pm 0.1$ & $0.7 \pm 0.1$ \\
\hline$a$ & 46 & $40 \pm 1$ & $1.0 \pm 0.2$ \\
$b$ & 73 & $78 \pm 1$ & $-0.55 \pm 0.11$ \\
$c$ & 1.06 & $1.02 \pm 0.02$ & $0.30 \pm 0.15$ \\
\hline
\end{tabular}

superfluid densities and corresponding isotope effect coefficients are listed in Table 4.8. The temperature dependence of the plane and axis superfluid densities were calculated similarly from the temperature dependent susceptibility data and are plotted in Figs. 4.13 and 4.14.

The results show that the $b$-axis superfluid density is greatest. This is to be expected due to proximity-induced condensation of carriers on the double $\mathrm{CuO}$ chains that extend in the $b$ direction[112]. The $c$-axis superfluid density is weakest. This is because the planes are only weakly (Josephson) coupled in the $c$-direction. Turning to the isotope effect we see that the isotope effect in the $b c$-plane is negligible compared with those in the $a b$ - and $a c$-planes. Assuming that this is a valid result, inspection of the phonon modes[114] shows that, with the exception of the $\mathrm{CuO}_{2}$ plane breathing modes, the motion of the atoms are parallel to the $b c$-plane. The implication is that the electrons prefer to couple to phonon modes in which the atomic motion is perpendicular to the transport plane. Support for this conjecture comes from site-selective oxygen isotope exchange experiments performed on $\mathrm{Y}_{0.6} \mathrm{Pr}_{0.4} \mathrm{Ba}_{2} \mathrm{Cu}_{3} \mathrm{O}_{7-\delta}[115]$. By preparing samples in which ${ }^{18} \mathrm{O}$ was substituted onto only either the planar oxygen sites or the apical and chain oxygen 

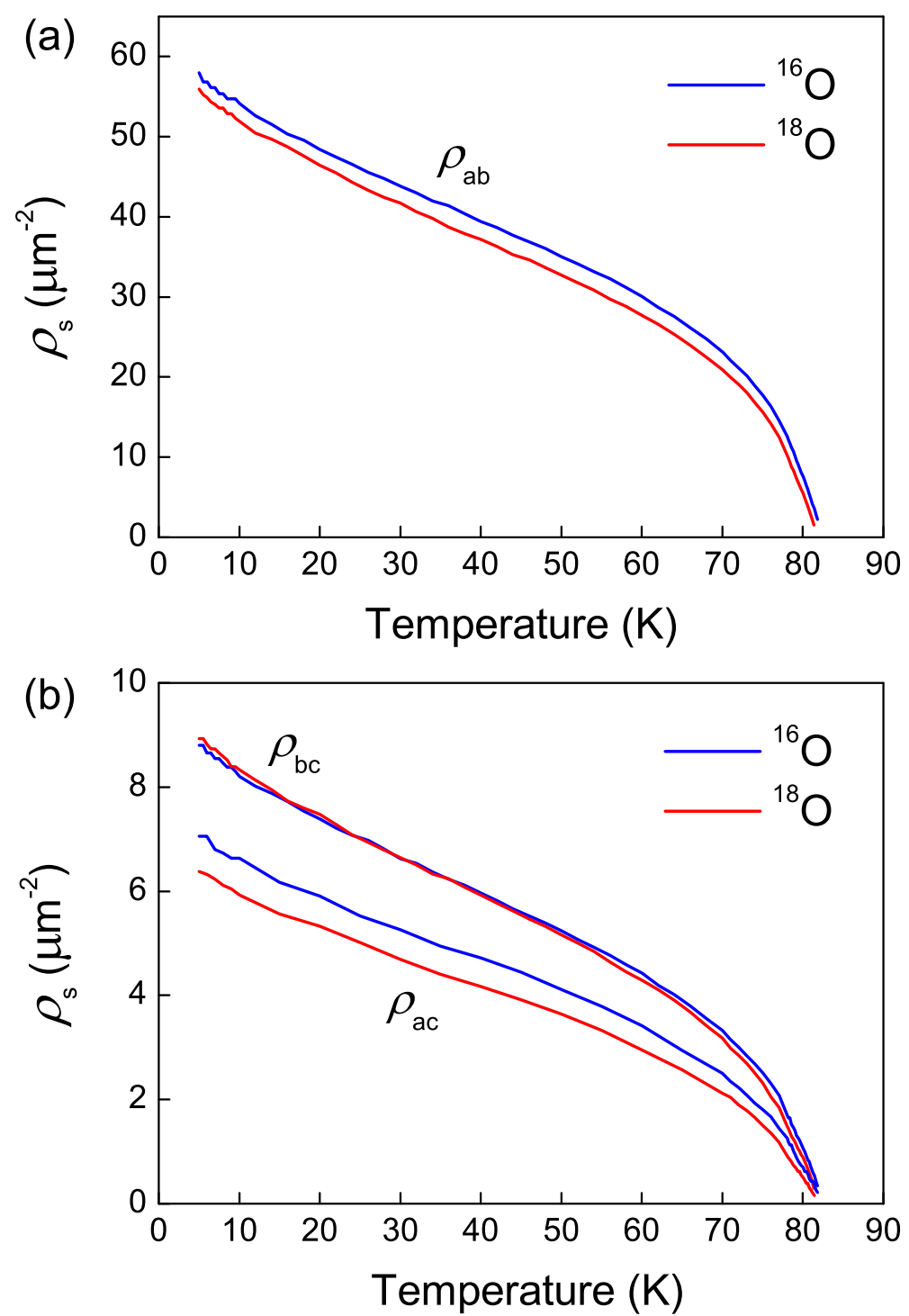

Figure 4.13: Superfluid density in the (a) $a b$-plane and (b) $b c$ - and $a c$-planes of ${ }^{16} \mathrm{O} /{ }^{18} \mathrm{O}$ substituted $\mathrm{Y}-124$. 

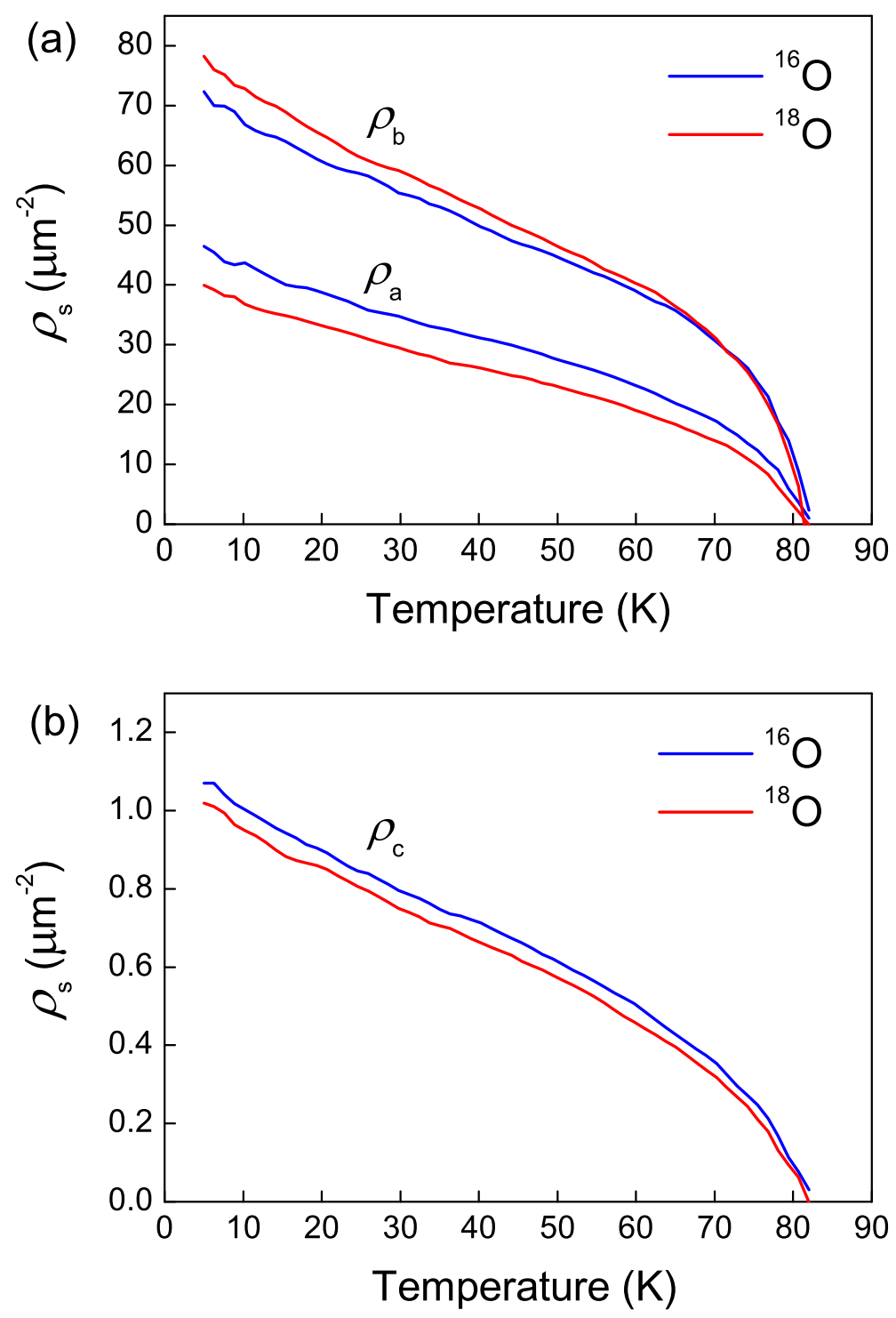

Figure 4.14: Superfluid density along the (a) $a$ - and $b$-axes (b) $c$-axis of ${ }^{16} \mathrm{O} /{ }^{18} \mathrm{O}$ substituted Y-124. 
sites;

"It was found that the whole or nearly the whole [isotope] effect on the transition temperature $T_{c}$ and on the in-plane magnetic penetration depth $\lambda_{a b}^{-2}$ (about $100 \%$ within error bars) comes from the oxygen within the superconducting $\mathrm{CuO}_{2}$ planes and not from the apical and chain oxygen." [115]

For an array of weakly coupled superconducting layers the out-of-plane (c-axis) penetration depth is[116]

$$
\lambda_{c}^{2}=\rho_{s, c}^{-2}=\frac{\hbar c^{2}}{8 \pi d e J_{c}}
$$

where $d$ is the interplanar spacing. $J_{c}$ is the critical current given by $[117,118]$

$$
J_{c}=\frac{\pi \Delta(T)}{2 e R_{n}} \tanh \left[\frac{\Delta(T)}{2 k T}\right]
$$

where $\Delta(T)$ is the superconducting gap and $R_{n}$ is the normal state resistance of the interlayer junctions. For a $d$-wave superconductor in the weak-coupling limit $\Delta(0)=2.14 k_{B} T_{c}$. Substituting for $\Delta$ and differentiating Eqn. 4.6 with respect to $T_{c}$ gives

$$
\begin{aligned}
\frac{1}{\rho_{s, c}} \frac{\partial \rho_{s, c}}{\partial T_{c}} & =\frac{1}{T_{c}}+\frac{1.07}{T \tanh \left(\frac{1.07 T_{c}}{T}\right)}-\frac{1.07 \tanh \left(\frac{1.07 T_{c}}{T}\right)}{T} \\
& =\frac{1}{T_{c}}, \text { as } T \rightarrow 0
\end{aligned}
$$


By definition

$$
\alpha\left(\rho_{s, c}\right)=-\frac{M}{\rho_{s}} \frac{\partial \rho_{s, c}}{\partial M}
$$

and

$$
\alpha\left(T_{c}\right)=-\frac{M}{\rho_{s}} \frac{\partial T_{c}}{\partial M}
$$

so in the $T=0$ limit

$$
\alpha\left(\rho_{s, c}\right)=-\left(\frac{T_{c}}{\rho_{s, c}} \frac{\partial \rho_{s, c}}{\partial T_{c}}\right) \frac{M}{T_{c}} \frac{\partial T_{c}}{\partial M}=\alpha\left(T_{c}\right)
$$

And it follows that

$$
\alpha\left(\lambda_{c}\right)=-\frac{1}{2} \alpha\left(T_{c}\right)
$$

The value of $\alpha\left(\rho_{s, c}\right)=0.3$ obtained in this experiment is nearly an order of magnitude larger than $\alpha\left(T_{c}\right)=0.046$ and is clearly inconsistent with the expected result given by Eqn. 4.11. This leads us to the conclusion that, for at least $\mathrm{YBa}_{2} \mathrm{Cu}_{4} \mathrm{O}_{8}$, the interlayer coupling is stronger than, or different from, simple Josephson coupling. This particular, and unexpected result, may reflect the unusual $c$-axis transport in Y-124 which at low temperatures is coherent[119] and when superconductivity is suppressed using strong magnetic fields remains metallic-like to the lowest temperatures (20K) unlike any other HTS system[120]. This indicates 3D band conductivity rather than the tunneling conductivity seen in other cuprates and is to be associated with the double $\mathrm{Cu}_{2} \mathrm{O}_{2}$ chains. It is not surprising that the Josephson coupling isotope effect is not seen in Y-124, though it should be seen in all other cuprates. 


\section{Chapter 5}

\section{Computational Techniques}

The calculations of physical properties in the next chapter were performed using software programs written entirely by the author. The programs were initially implemented in the free $J a v a^{T M}$ programming language. As the complexity of the calculations increased the programs were translated to the $C++$ programming language. $C++$ applications are compiled to native machine code and so offer a speed advantage over Java applications that are interpreted by a runtime environment. The programs were compiled using the free Borland® $C++$ Compiler 5.5. The calculations were performed on a desktop P.C. with an Intel $\AA$ Pentium $\AA 4$ dual core CPU having a combined clock speed of $3.00 \mathrm{GHz}$.

An object-oriented (or modular) approach was taken with regards to the design of the programs. Such an approach allows code to be easily modified, extended and reused. An object-oriented program consists of a collection of cooperating objects. An object is a bundle of variables and related methods (a method is similar to a procedure or function), and therefore has a particular 


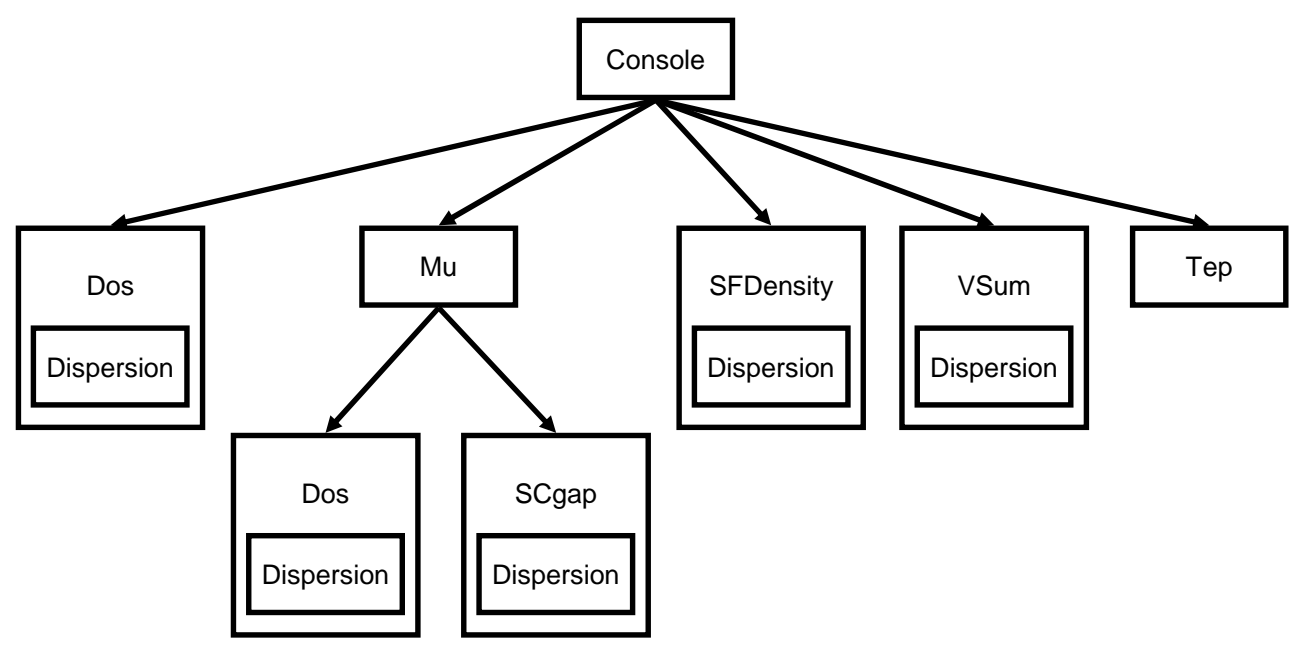

Figure 5.1: The Console program architecture.

state and behaviour. Each object is an instance of a particular class of objects. A class is the blueprint from which individual objects are created, and defines the states and behaviours that those objects may possess.

Before discussing each of the classes individually we list for convenience the key equations for which these classes were written to evaluate. They are:

1. The density of states.

$$
N(E)=N_{k}^{-1} \sum_{\mathbf{k}} \delta[\epsilon(\mathbf{k})-E]
$$

where $\epsilon(\mathbf{k})$ is the energy-momentum dispersion.

2. The BCS gap equation.

$$
1=\frac{V}{2} \sum_{\mathbf{k}} \frac{|g(\mathbf{k})|^{2}}{E(\mathbf{k})} \tanh \left(\frac{E(\mathbf{k})}{2 k_{B} T}\right)
$$


where $E(\mathbf{k})=\sqrt{\epsilon^{2}(\mathbf{k})+\Delta^{2}(\mathbf{k})}$

3. The number of carriers.

$$
n=\left(2 / V_{A}\right) \int f(E) N(E) d E
$$

where $f(E)=\left(\exp \left(\frac{E-\mu(T)}{k_{B} T}\right)+1\right)^{-1}$

4. The superfluid density.

$$
\begin{aligned}
\frac{1}{\lambda_{a b}^{2}} & =\frac{\mu_{0} e^{2} n}{4 \pi \hbar^{2}} \sum_{\mathbf{k}}\left[\left(\frac{\partial \epsilon(\mathbf{k})}{\partial k_{x}}\right)^{2} \frac{\Delta^{2}(\mathbf{k})}{E^{2}(\mathbf{k})}-\frac{\partial \epsilon(\mathbf{k})}{\partial k_{x}} \frac{\partial \Delta(\mathbf{k})}{\partial k_{x}} \frac{\Delta(\mathbf{k}) \epsilon(\mathbf{k})}{E^{2}(\mathbf{k})}\right] \\
& \times\left[\frac{1}{E(\mathbf{k})}-\frac{\partial}{\partial E(\mathbf{k})}\right] \tanh \left(\frac{E(\mathbf{k})}{2 k_{B} T}\right)
\end{aligned}
$$

5. The diffusion thermopower.

$$
S(T)=\frac{1}{|e| T} \frac{\int_{-\infty}^{\infty} \sigma(E)(E-\mu)\left(\frac{\partial f(E)}{\partial E}\right) d E}{\int_{-\infty}^{\infty} \sigma(E)\left(-\frac{\partial f(E)}{\partial E}\right) d E}
$$

$\sigma(E)$ is the spectral conductivity which under the Boltzmann formalism is given by

$$
\sigma(E)=\frac{e^{2}}{V} \sum_{\mathbf{k}} v_{\alpha}(E, \mathbf{k}) \ell_{\alpha}(E, \mathbf{k}) \delta[\epsilon(\mathbf{k})-E]
$$

where $\ell_{\alpha}=v_{\alpha}(E, \mathbf{k}) \cdot \tau(E, \mathbf{k}, T)$ and $v_{\alpha}=\frac{\partial \epsilon(\mathbf{k})}{\partial k_{\alpha}}$

A more thorough expanation of the terms in each of the equations can be found in the following chapter. 


\subsection{Dispersion}

The Dispersion class forms a base class for the Dos, SCgap, SFDensity and VSum classes. The purpose of this class is to return information about the electronic dispersion. Dispersion stores the tight binding coefficients of several cuprate energy-momentum dispersions and has methods for calculating the energy $\epsilon(\mathbf{k})$, pseudogap energy, superconducting gap energy $\Delta(\mathbf{k})$, and various derivatives of $\epsilon(\mathbf{k})$ and $\Delta(\mathbf{k})$, at any specified $\mathbf{k}$-space coordinate and temperature. The user is able to specify which dispersion to use, the position of the Fermi level, the pseudogap magnitude and the maximum angle to which the pseudogap extends around the Fermi surface.

\subsection{Dos}

The Dos class is derived from the Dispersion class, which means that it inherits the methods (functions) of the Dispersion class. It has methods for returning the density of states, number of carriers, spin susceptibility, electronic entropy and spin lattice relaxation rate for any specified dispersion, temperature, superconducting gap and pseudogap values.

The density of states (Eqn. 5.1) is determined by calculating the energy at each point of a 3000 by 3000 grid spanning a quadrant of the first Brillouin zone $\left(k_{x}=0 \rightarrow \pi, k_{y}=0 \rightarrow \pi\right)$. A histogram of the energies is stored in an array with an energy resolution of $1 \mathrm{meV}$. The array index $i$ is calculated according to $i=\left(E-E_{1}\right) / d E$ where $d E=1 \mathrm{meV}$ and $E_{1}$ is the lower energy bound. If $i$ is fractional it is rounded to the nearest integer. The density 
of states can be output as a tab-delimited text file. The number of carriers, spin susceptibility, electronic entropy and spin lattice relaxation rate are calculated by performing numerical integrations of the density of states using the trapezium rule.

An instance of the Dos class, known as a Dos object, is utilized by the $\mathrm{Mu}$ class in calculating the temperature-corrected chemical potential.

\subsection{SCgap}

The SCgap class is derived from the Dispersion class. The purpose of this class is to calculate the superconducting gap self consistently from the BCS gap equation (Eqn. 5.2) at a given temperature and chemical potential.

The superconducting gap amplitude, $\Delta_{0}$, is calculated recursively until it stabilises to within 0.1 percent. The trial values for $\Delta_{0}$ in each recursive step are determined by linear extrapolation of the two previous trial values, as illustrated in Fig 5.2. In order to improve speed the k-space sum is performed over a smaller 1000 by 1000 grid spanning a quadrant of the first Brillouin zone.

An instance of the SCgap class, known as an SCgap object, is utilized by the $\mathrm{Mu}$ class in calculating the temperature-corrected chemical-potential.

\section{$5.4 \mathrm{Mu}$}

The Fermi energy is the value of the chemical potential at zero temperature. The primary purpose of the Mu class is to determine the temperature- 


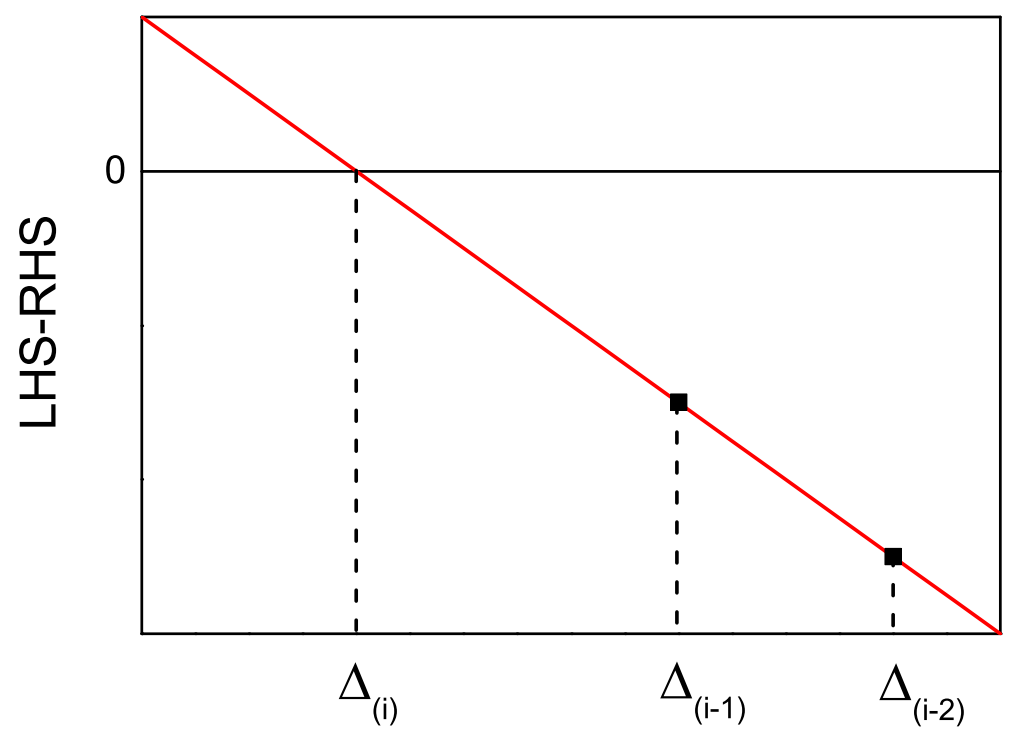

Figure 5.2: The correct value of the superconducting gap magnitude, $\Delta_{0}$, results in equality between both sides of the BCS gap equation. Trial values of $\Delta_{0}$ are chosen by linear extrapolation of the two preceding trial values.

corrected chemical potential, $\mu(T)$, for a given energy-momentum dispersion and Fermi energy. To do this the Mu class enlists the services of a Dos object and an SCgap object.

$\mu$ is calculated recursively so that the number of carriers remains constant (to within 0.001 percent) with temperature, a condition defined by Eqn. 5.3. The number of carriers is obtained from the Dos object. The algorithm used in determining $\mu$ is illustrated in Fig. 5.3. The trial values for $\mu$ in each recursive step are determined by linear extrapolation of the two preceding trial values.

In the absence of both a pseudogap and superconducting gap the calculation of $\mu(T)$ is very fast as only a single calculation of the density of states is required. However, in the presence of a pseudogap and/or supercon- 
ducting gap, the density of states must be re-computed whenever $\mu$ changes. This is because the pseudogap is pinned to the chemical potential and the superconducting gap energy (obtained from the SCgap object) is calculated self-consistently. For a given temperature $\mu$ can usually be determined in 3-4 iterations.

Once the chemical potential at a given temperature has been determined the $\mathrm{Mu}$ class obtains the susceptibility, electronic entropy and spin lattice relaxation time from the Dos object. These quantities along with the superconducting gap amplitude data obtained from the SCgap object are output to a tab-delimited text file by the Mu class. In addition, binary files are produced contaning the $\mu(T)$ and $\Delta_{0}(T)$ data. These files are loaded as inputs by the SFDensity, VSum and Tep classes.

\subsection{SFDensity}

The SFDensity class is derived from the Dispersion class. It computes the superfluid density from Eqn. 5.4. The $\mathbf{k}$-space sum is performed over a 1000 by 1000 grid spanning a quadrant of the first Brillouin zone. As inputs, the class loads the two binary files produced by the Mu class which contain the temperature dependent chemical potential and superconducting gap amplitude data. The superfluid density as a function of temperature is written to a tab-delimited text file. 


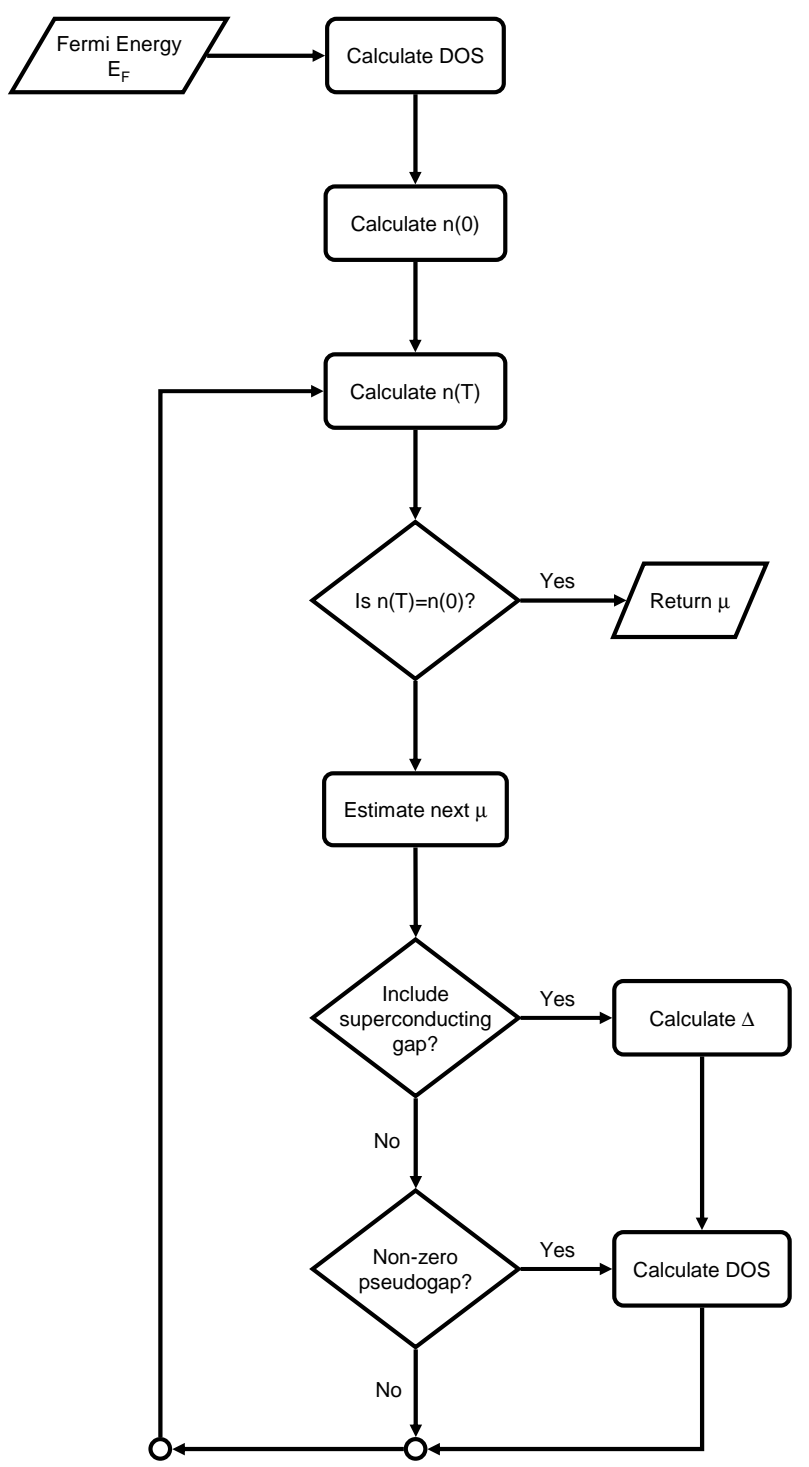

Figure 5.3: The algorithm used in determining the chemical potential, $\mu$, as a function of temperature. $n$ is the number of carriers. 


\subsection{VSum}

The VSum class is derived from the Dispersion class. The purpose of this class is to perform intermediate calculations that are then used by the Tep class in calculating the thermoelectric power. Specifically VSum loads the chemical potential binary file produced by the $\mathrm{Mu}$ class as an input and performs the following $\mathbf{k}$-space sum at each temperature

$$
V(E, T)=\sum_{\mathbf{k}} v_{x}^{2} \delta[\epsilon(\mathbf{k})-\mu(T)-E]
$$

where $v_{x}=\frac{\partial \epsilon(\mathbf{k})}{\partial k_{x}}$. The sum is performed at each point of a 3000 by 3000 grid spanning a quadrant of the first Brillouin zone. The $V(E, T)$ table is stored in a two-dimensional array indexed by temperature and energy. The energy resolution is $1 \mathrm{meV}$. The calculation is performed in much the same way as the density of states. The only difference is that instead of incrementing the value stored in a particular cell by 1 we increment the value by $v_{x}^{2}$. VSum outputs both a tab-delimited text file and binary file containing the $V(E, T)$ table. The electrical conductivity, $\sigma$, in the Boltzmann formalism can be obtained by multiplying $V(E, T)$ by the scattering rate $\tau(E, T)$.

\subsection{Tep}

The Tep class loads the chemical potential and velocity sum binary files produced by the $\mathrm{Mu}$ and VSum classes as inputs and computes the thermoelectric power according to Eqn. 5.5. The form of the scattering rate, $\tau(E, T)$, can be chosen by the user. 


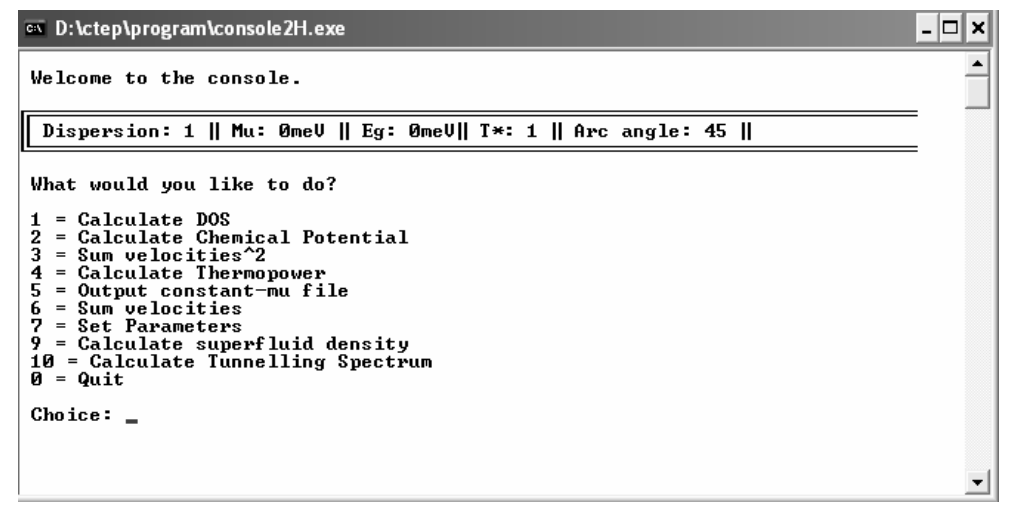

Figure 5.4: A screen capture of the Console program interface.

\subsection{Console}

Console is the main class of the program and provides a text-based user interface. Where possible textual menus are presented to the user. A screen-shot of the main menu is shown in Fig. 5.4. A graphical user interface can be added simply by the replacement of this class. Its purpose is to prompt the user for the type of calculation to be performed and to ensure that a valid and complete set of input parameters is passed to each of the Dos, Mu, SFDensity, VSum and Tep objects. To conserve memory the Console class creates these objects dynamically for the duration of the calculation. Filenames are generated by the class based on the dispersion, Fermi energy and pseudogap parameters specified by the user. The Console program architecture is illustrated in Fig. 5.1. 


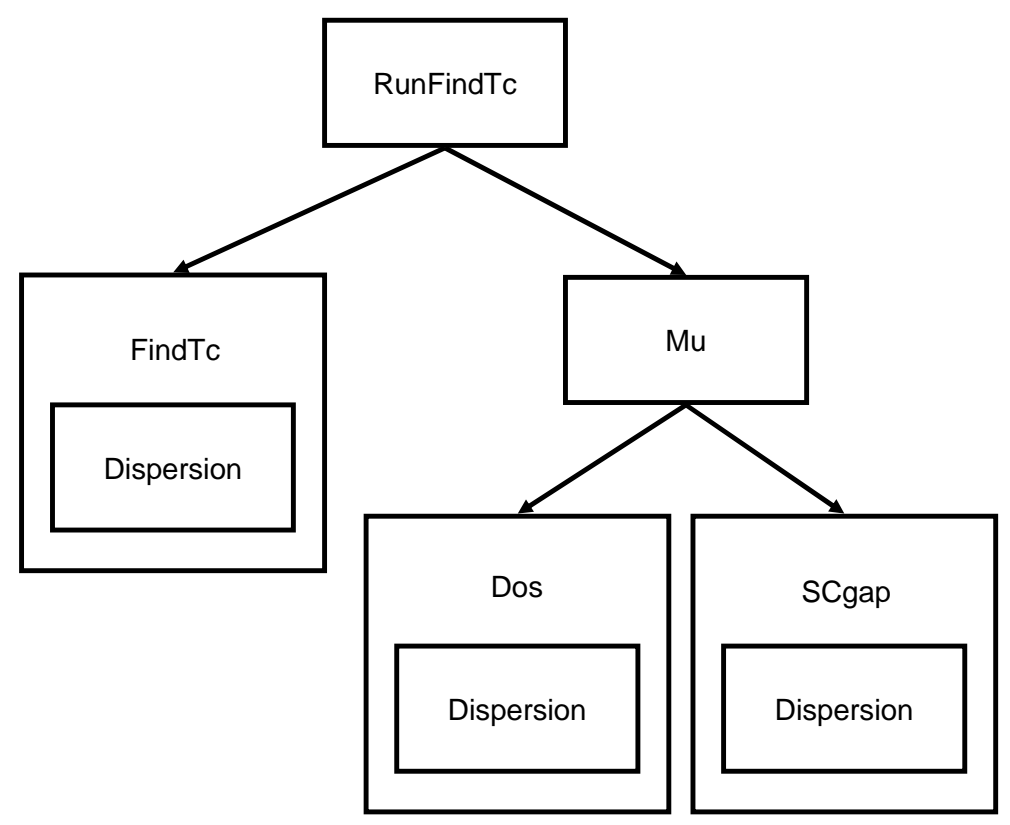

Figure 5.5: The RunFindTc program architecture.

\section{$5.9 \quad$ RunFindTc}

RunFindTc is a program written to calculate the transition temperature by numerically solving the self consistent BCS gap equation in the case where the superconducting gap magnitude is zero. The program architecture is shown in Fig. 5.5. The RunFindTc class obtains the required parameters from the user and which are then passed to instances of the FindTc and $\mathrm{Mu}$ classes. Using the outputs from these objects the transition temperature is calculated in such a way that includes corrections to the chemical potential for temperature. The algorithm is illustrated in Fig. 5.6. 


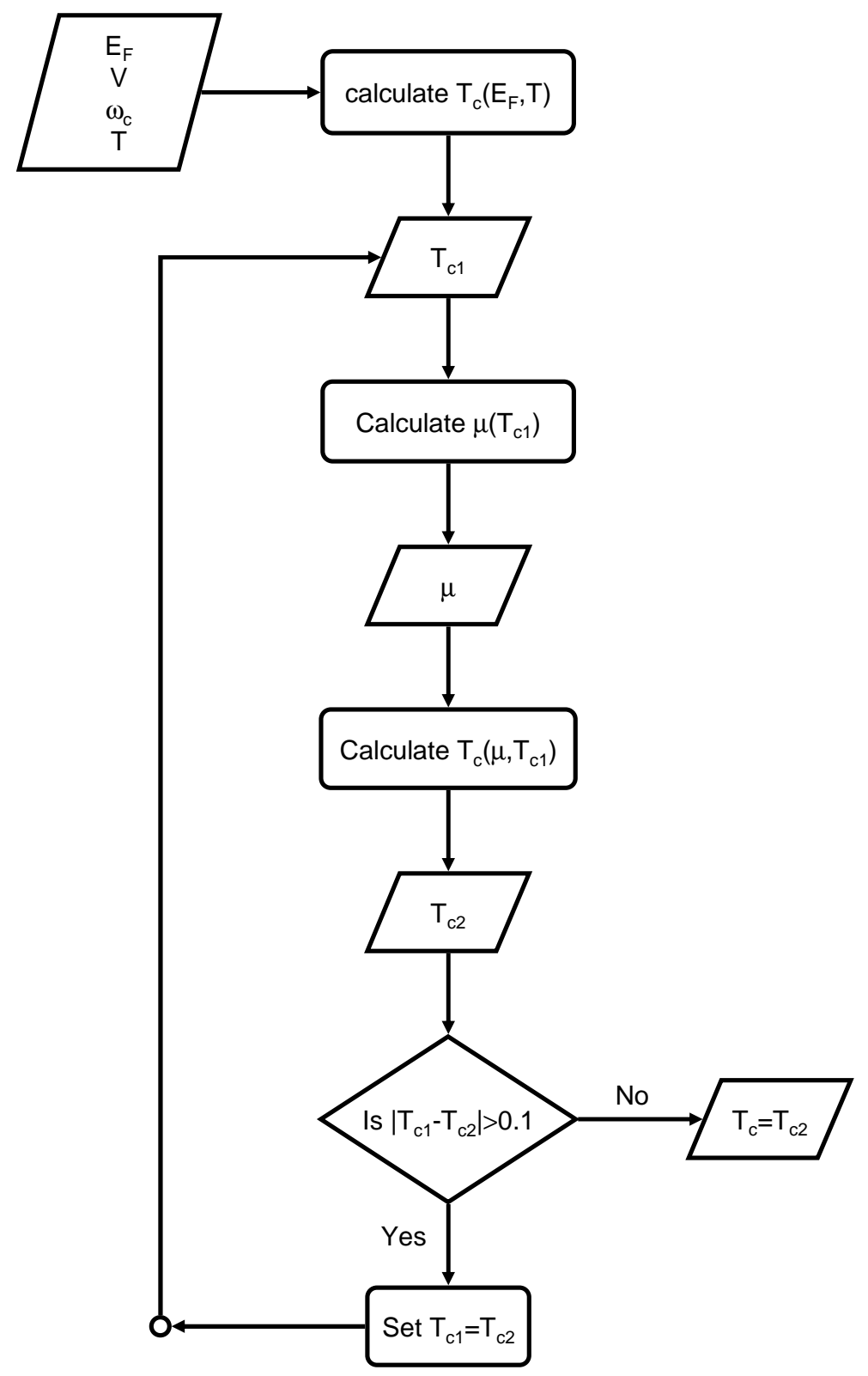

Figure 5.6: The algorithm used in determining the transition temperature $T_{c}$. 


\section{$5.10 \quad$ FindTc}

The FindTc class is derived from the Dispersion class. An instance of FindTc is used by the RunFindTc class in calculating the transition temperature. The FindTc class is very similar to the SCgap class. In the SCgap class the temperature is specified and a recursive algorithm is used to determine the superconducting gap magnitude from the self consistent BCS gap equation. In the FindTc class it is the superconducting gap magnitude that is specified $(=0)$ and the transition temperature is determined recursively. The class requires an initial guess from the user of the value of $T_{c}$. Subsequent trial values are then computed using a linear extrapolation method. The algorithm is unable to accommodate a temperature dependent pseudogap because the calculations become non-linear and therefore the linear extrapolation method no longer produces converging trial temperatures. 


\section{Chapter 6}

\section{Computational Results}

\subsection{Electronic Entropy}

For Bi-2212 we employ a two-dimensional bilayer dispersion $\epsilon(\mathbf{k})$ provided by the authors of Ref. [42]. It was obtained from tight binding fits to highresolution ARPES data from an overdoped sample with a $T_{c}$ of $55 \mathrm{~K}$. The tight binding basis functions and coefficients are listed in table 6.1. Similar fits made to data from a less overdoped sample with $T_{c}=80 \mathrm{~K}$ suggest that the hopping parameters change slightly with doping, but the error bars in the data are also larger for that sample. For our purposes we assume that the parameters are independent of doping and that the Fermi level shifts with doping relative to the "rigid" band structure.

The density of states (DOS) per spin at energy $E$ is given by

$$
N(E)=N_{k}^{-1} \sum_{\mathbf{k}} \delta[\epsilon(\mathbf{k})-E]
$$


Table 6.1: Bi-2212 bilayer dispersion tight binding basis functions and coefficients where $\epsilon(\mathbf{k})=\sum c_{i} \eta_{i}(\mathbf{k})$.

\begin{tabular}{|c|c|}
\hline$c_{i}$ & $\eta_{i}(\mathbf{k})$ \\
\hline 0.183 & 1 \\
-0.654 & $\frac{1}{2}\left(\cos k_{x}+\cos k_{y}\right)$ \\
0.1236 & $\cos k_{x} \cos k_{y}$ \\
-0.1317 & $\frac{1}{2}\left(\cos 2 k_{x}+\cos 2 k_{y}\right)$ \\
0.0083 & $\frac{1}{2}\left(\cos 2 k_{x} \cos k_{y}+\cos k_{x} \cos 2 k_{y}\right)$ \\
0.0212 & $\cos 2 k_{x} \cos 2 k_{y}$ \\
0.053 & $\pm \frac{1}{4}\left(\cos k_{x}-\cos k_{y}\right)^{2}$ \\
\hline
\end{tabular}

where $N_{k}$ is the number of $k$-space points over which the summation is performed. The entropy per mole for weakly interacting fermions is given by[121]

$$
S=-2 R \int[f \ln f+(1-f) \ln (1-f)] N(E) d E
$$

where $f$ is the Fermi-Dirac distribution function and $R$ is the gas constant. The chemical potential $\mu(T)$ is calculated self-consistently such that the carrier concentration $n$ is $T$-independent. $n$ is given by:

$$
n=\left(2 / V_{A}\right) \int f(E) N(E) d E
$$

Where $V_{A}$ is the atomic volume per formula unit.

The Fermi surface (FS) in the 1st Brillouin Zone is shown in the inset to Fig. 6.3. The pseudogap first forms on the FS near $(\pi, 0)$ leaving ungapped Fermi arcs[26] between. With decreasing temperature the Fermi arcs narrow such that the gap becomes nodal at $T=0$. We therefore adopt a pseudogap 
of the form

$$
E_{g}= \begin{cases}E_{g, \text { max }} \cos \left(\frac{2 \pi \theta}{4 \theta_{0}}\right), & \left(\theta<\theta_{0}\right) \\ E_{g, \text { max }} \cos \left(\frac{2 \pi(\theta-\pi / 2)}{4 \theta_{0}}\right), & \left(\theta>\frac{\pi}{2}-\theta_{0}\right) \\ 0 & \text { otherwise }\end{cases}
$$

where

$$
\theta_{0}=\frac{\pi}{4}\left[1-\tanh \left(\frac{T}{T^{*}}\right)\right]
$$

and $T^{*}=E_{g, \max } / k_{B} . \quad 0 \leq \theta \leq \pi / 2$ is the angle shown in Fig. 6.3. The pseudogap is states-non-conserving i.e. unlike the SC gap there is no pile up of states outside the gap (see Fig. 6.3). This is implemented by eliminating states with energies $E<E_{g}$ from the summations. A plot of the antibonding band energy-momentum dispersion with a $10 \mathrm{meV}$ pseudogap at $50 \mathrm{~K}$ is shown in Fig. 6.1. The evolution with temperature of the pseudogap on the Fermi surface is shown in Fig. 6.2.

Fig. 6.3 shows the DOS calculated from the bilayer dispersion. The bonding and antibonding band vHs's are clearly visible with the former $105 \mathrm{meV}$ below the latter. Also shown is a $20 \mathrm{meV}$ pseudogap at $T=0 \mathrm{~K}$ and $100 \mathrm{~K}$ illustrating the gap filling with temperature. The gap node is pinned to the chemical potential at all $T$.

Eqn. 6.5 models the observed temperature dependence of the Fermi arc length[72]. At $T=0, \theta_{0}=\pi / 4$ and the pseudogap is fully nodal. As the temperature rises, $\theta_{0}$ decreases resulting in a 'filling-in' of the pseudogap and 


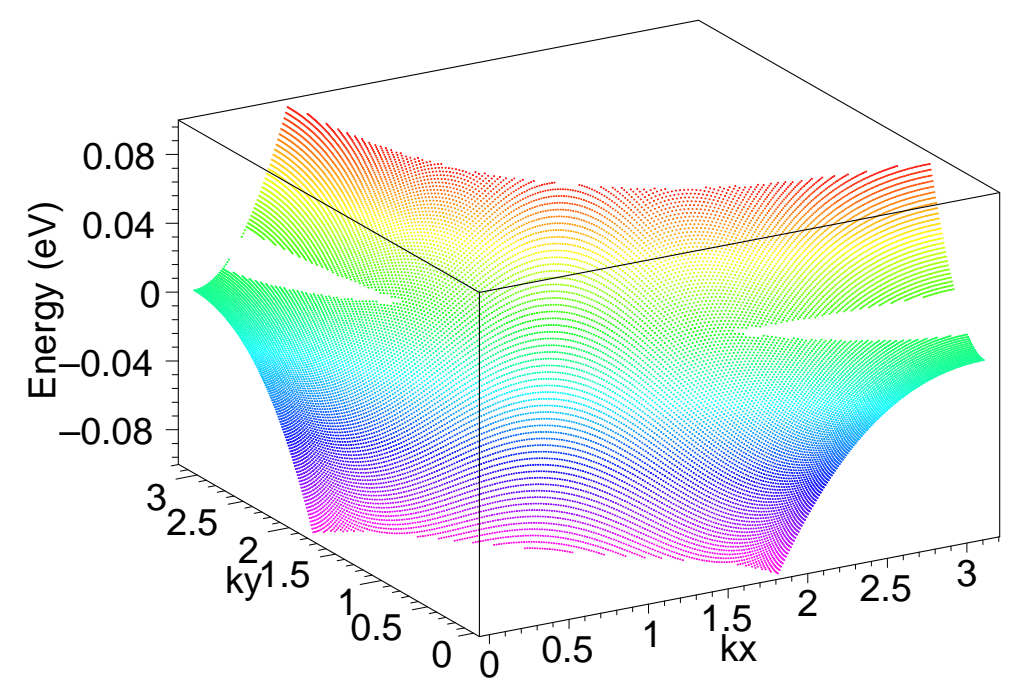

Figure 6.1: The antibonding band energy-momentum dispersion with a $10 \mathrm{meV}$ pseudogap at $50 \mathrm{~K}$. The gap nodes retreat to the zone edges with increasing temperature.

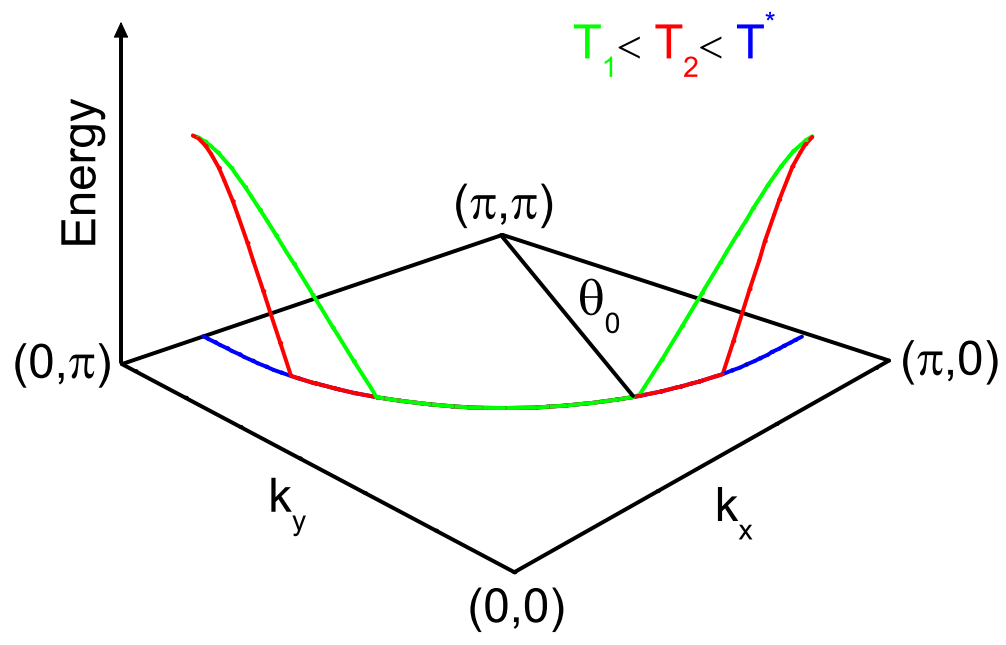

Figure 6.2: The evolution of the pseudogap with temperature as defined by Eqns. 6.4 and 6.5 on Fermi surface (blue). The ungapped sections of the Fermi surface are referred to as "Fermi arcs". 


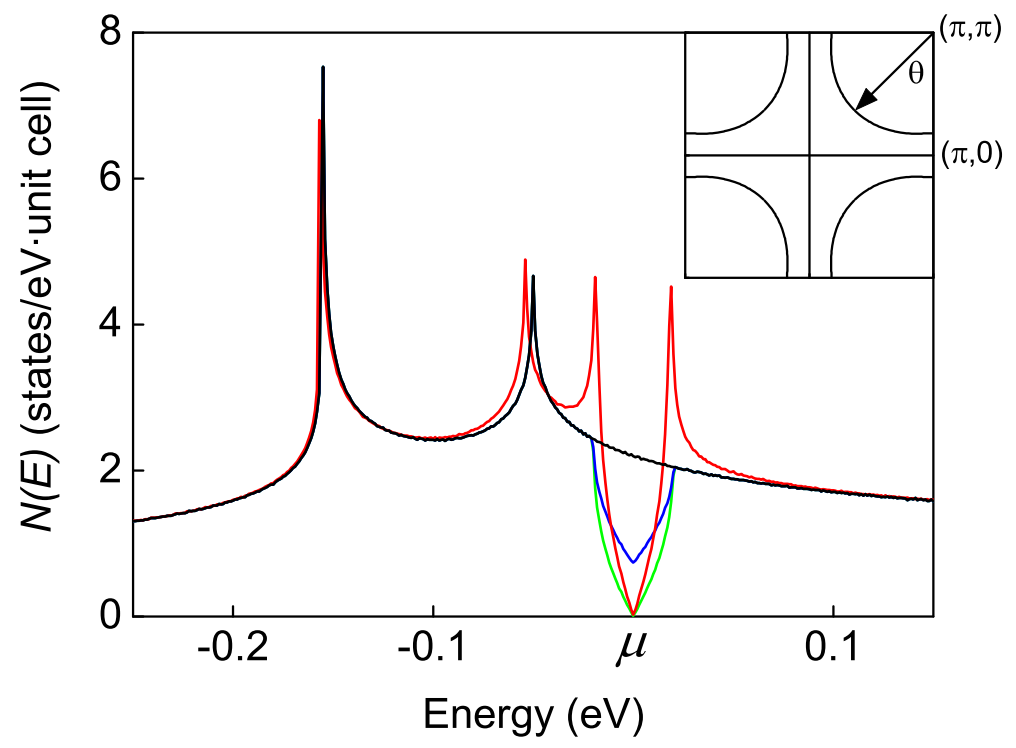

Figure 6.3: The density of states calculated from the Bi-2212 bilayer dispersion determined by ARPES measurements (black). Also shown is a $20 \mathrm{meV}$ pseudogap at $0 \mathrm{~K}$ (green) and $100 \mathrm{~K}$ (blue), and a $20 \mathrm{meV} \mathrm{SC}$ gap at $0 \mathrm{~K}$ (red). Inset: the Fermi surface in the $\left(k_{x}, k_{y}\right)$ plane showing the angle $\theta$. 
the growth of the Fermi-arcs. This model is based on results by Kanigel et al.[72] that show the Fermi-arcs collapsing linearly as a function of $T / T^{*}$, extrapolating to zero as $T \rightarrow 0$. However we note an important difference between this model and their results. The Kanigel data shows the pseudogap opening abruptly at $T=T^{*}$. A pseudogap which fills completely at $T^{*}$ would result in a jump in the specific heat coefficient $\gamma$ at $T^{*}$, which is not observed experimentally (see Fig. 6.4). The smooth evolution of the tanh function in Eqn. 6.5 overcomes this problem.

We suggest that the apparent closing of the pseudogap is an artifact of quasiparticle lifetime broadening. Kanigel et al. estimate the size of the pseudogap around the Fermi surface as being half the peak to peak separation in the symmetrized ARPES quasiparticle energy dispersion curves (EDCs). The symmetrized ARPES EDCs have been simulated[123] as the sum of two thermally broadened Lorentzians. The simulations (see Fig. 6.5) show that estimating the gap size in this fashion results in an apparent gap that fills faster than the actual gap. The discrete jump at $T^{*}$ in the Fermi arc length reported by Kanigel et al. is also found to be an artifact of their peak-topeak analysis in the presence of quasiparticle lifetime broadening. Thus, as shown in inset (a) of Fig. 6.5, a true $d$-wave gap $(\propto \cos 2 \phi)$ with nodes at $\phi=45^{\circ}$ appears to close at a smaller angle, exposing apparent, but fictitious, Fermi arcs. The inset (b) to Fig. 6.5 shows the temperature dependence of the apparent Fermi arc length (FAL). This seems to extend out abruptly to cover the entire Fermi surface at $T^{*}$, just as reported by Kanigel et al. (see data points in inset (b)). But the entire effect is fictitious in that it can be caused by quasiparticle lifetime effects. 

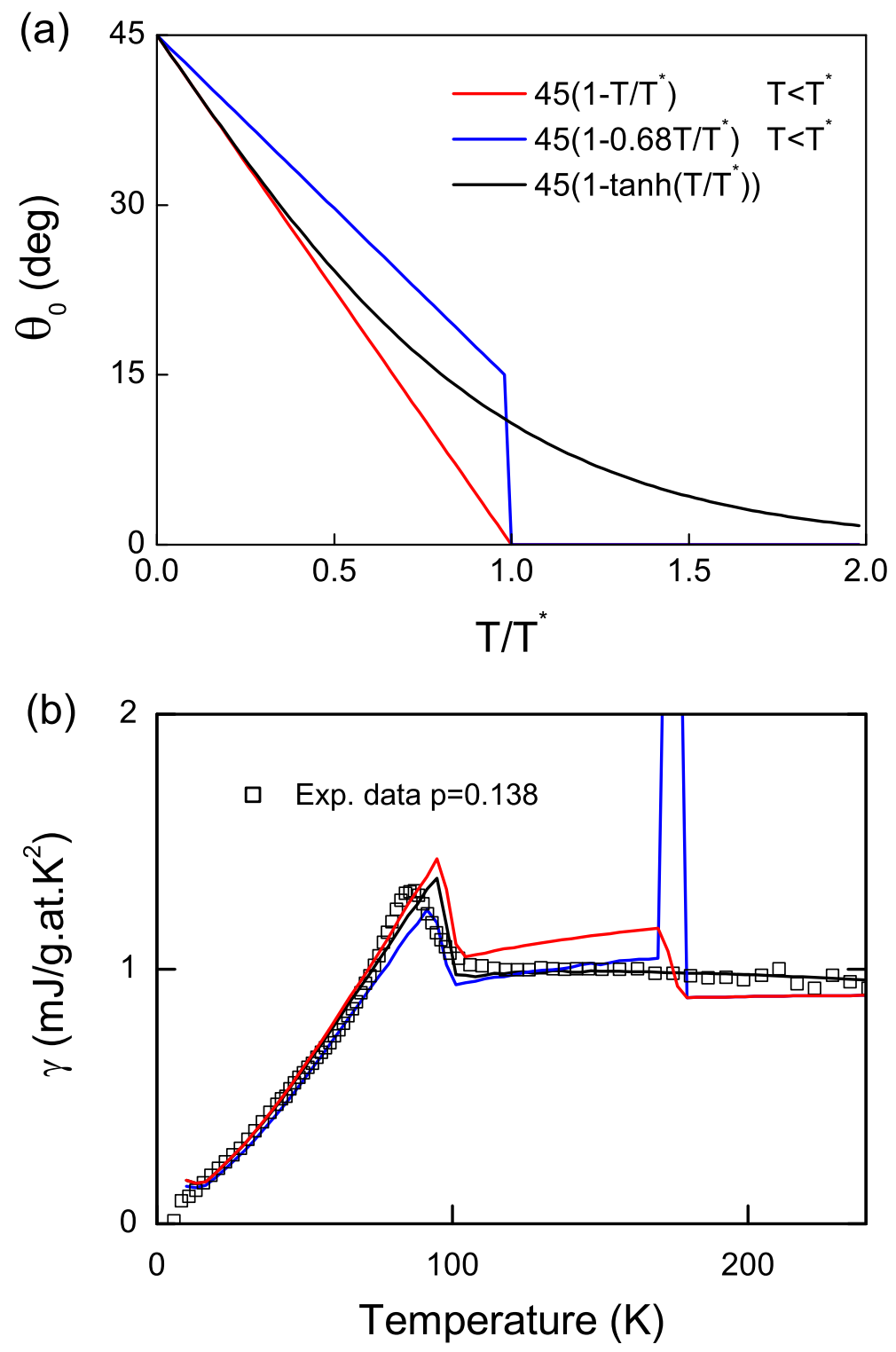

Figure 6.4: (a) The temperature dependence of $\theta_{0}$ for three scenarios: (i) the pseudogap fills linearly up to $T^{*}$ (red); (ii) the filling behaviour deduced from ARPES by Kanigel et al. [72] displaying a discrete jump at $T^{*}$ (blue); (iii) an asymptotically filling pseudogap (black). (b) The specific heat coefficient calculated for the above scenarios and compared with experimental data for Bi-2212[122] where every 10th data point is shown. 


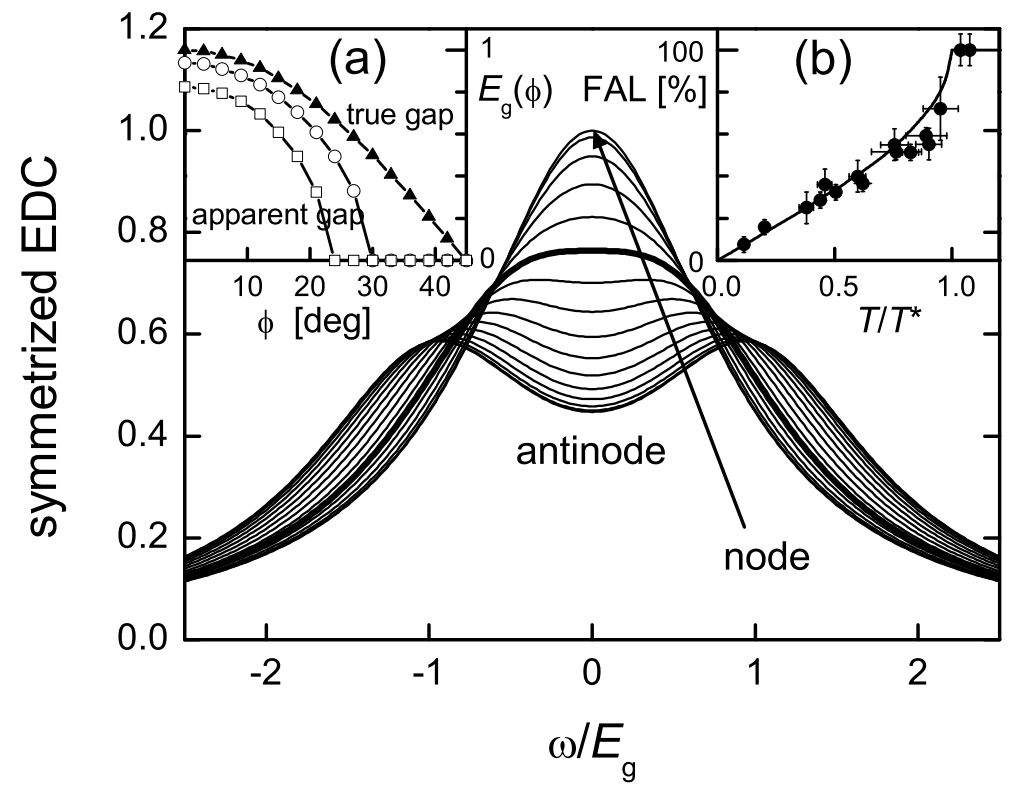

Figure 6.5: Simulated symmetrized ARPES quasiparticle EDCs ranging from the antinode to the node $\left(\theta=0\right.$ to $\left.45^{\circ}\right)[123]$. The broadening is fixed at $0.45 E_{g}(0)$. The crossover from double to single peaks (bold curve) presents an apparent, though false, closing of the PG and recovery of Fermi arcs. Inset (a) true normalized gap and apparent gap for two broadenings $\left[=0.45 E_{g}(0)\right.$ and $\left.0.6 E_{g}(0)\right]$. (b) curve: apparent Fermi arc length (FAL) assuming broadening $\propto T / T^{*}$; data: from Ref. [72] 
The entropy in the SC state has been modelled using a $d$-wave gap of the form $\Delta(\mathbf{k})=\frac{1}{2} \Delta_{0} g(\mathbf{k})$ where $g(\mathbf{k})=\cos k_{x}-\cos k_{y}$. The dispersion in the presence of the SC gap is given by $E(\mathbf{k})=\sqrt{\epsilon^{2}(\mathbf{k})+\Delta^{2}(\mathbf{k})}$ and $\Delta_{0}(T)$ is determined from the self-consistent weak-coupling BCS gap equation[124]

$$
1=\frac{V}{2} \sum_{\mathbf{k}} \frac{|g(\mathbf{k})|^{2}}{E(\mathbf{k})} \tanh \left(\frac{E(\mathbf{k})}{2 k_{B} T}\right)
$$

The summation is performed over both the bonding and anti-bonding bands and $\Delta$ is assumed to be the same for both bands[125]. We adopt a pairing potential of the form $V_{\mathbf{k k}^{\prime}}=V g(\mathbf{k}) g\left(\mathbf{k}^{\prime}\right)$. The amplitude, $V$, is assumed to be constant $(=125 \mathrm{meV})$ up to an energy cut-off, $\omega_{c}$, chosen such that $T_{c}$ matches the experimentally observed value. The pseudogap is not included in the process of calculating $\Delta_{0}(T)$. Including the pseudogap as modelled by Eqn. 6.4 in the self-consistent calculation of $\Delta_{0}(T)$ quickly results in $\Delta_{0}$ going to zero for $E_{g}$ values that are much smaller than what is experimentally observed. The reason for this can be explained as follows.

In the case of an $s$-wave superconducting gap the BCS gap equation takes the form[3]

$$
1=N(0) V \int_{0}^{\omega_{c}} \frac{d \epsilon}{\sqrt{\epsilon^{2}(\mathbf{k})+\Delta^{2}(\mathbf{k})}}
$$

In the pseudogap model, states with energies less than $E_{g}$ are eliminated. These low-energy states provide the highest contributions to the integral. In order to compensate, $\Delta(\mathbf{k})$ reduces. The rapid reduction in $\Delta(\mathbf{k})$ with increasing $E_{g}$ is due to the states-non-conserving nature of the model. In order to incorporate the pseudogap self-consistently under the current scheme 
we are left with two possiblities: (a) introducing a reduction in $E_{g}$ at low temperatures; (b) altering the zero-temperature Fermi-arc length. These possibilities will be explored in sections 6.2 and 6.3 .

Fig. 6.6(a) shows the experimental normal- and SC-state entropy data of Loram et al[122]. Fig. 6.6(b) shows the absolute entropy calculated from dispersion with no fitting parameters. We have merely specified the location of $E_{F}$ relative to $E_{v H s}$ at two points only: $0 \mathrm{meV}$ for an overdoped sample with $p=0.225[42] ; 96 \mathrm{meV}$ for an underdoped sample with $p=0.11[126]$; and interpolated between (as well as a little beyond the vHs). The doping dependence of $E_{g}$ is obtained from measurements of the leading-edge ARPES gap at $100 \mathrm{~K}[127]$. The overall $T$ - and doping-dependence of the experimental data is reproduced superbly, with absolute values just a factor of $3 / 4$ lower. In Fig. 6.6(a) we have rescaled the computed entropy by a constant factor of $4 / 3$ and refined the fit by using $E_{F}$ and $E_{g, \max }$ as fitting parameters. These refinements do not alter the overall behaviour and are tightly constrained. For example, the normal-state fits to the four most overdoped data sets have been obtained by adjusting a single parameter, namely $E_{F}$, as are the high- $T$ asymptotes for all data sets. The Fermi level in the most overdoped fit is only $8 \mathrm{meV}$ above the antibonding band vHs.

As the doping decreases the $\mathrm{vHs}$ recedes from $E_{F}$ resulting in a decrease in the number of states within $k_{B} T$ of $\mu$ and a corresponding reduction in entropy. However, as the doping is further reduced the recession of the vHs from $E_{F}$ is no longer able to account for the observed decrease in entropy alone and the second adjustable parameter, the pseudogap magnitude $E_{g}$, is introduced. This results in the progressive downturn in the normal state 


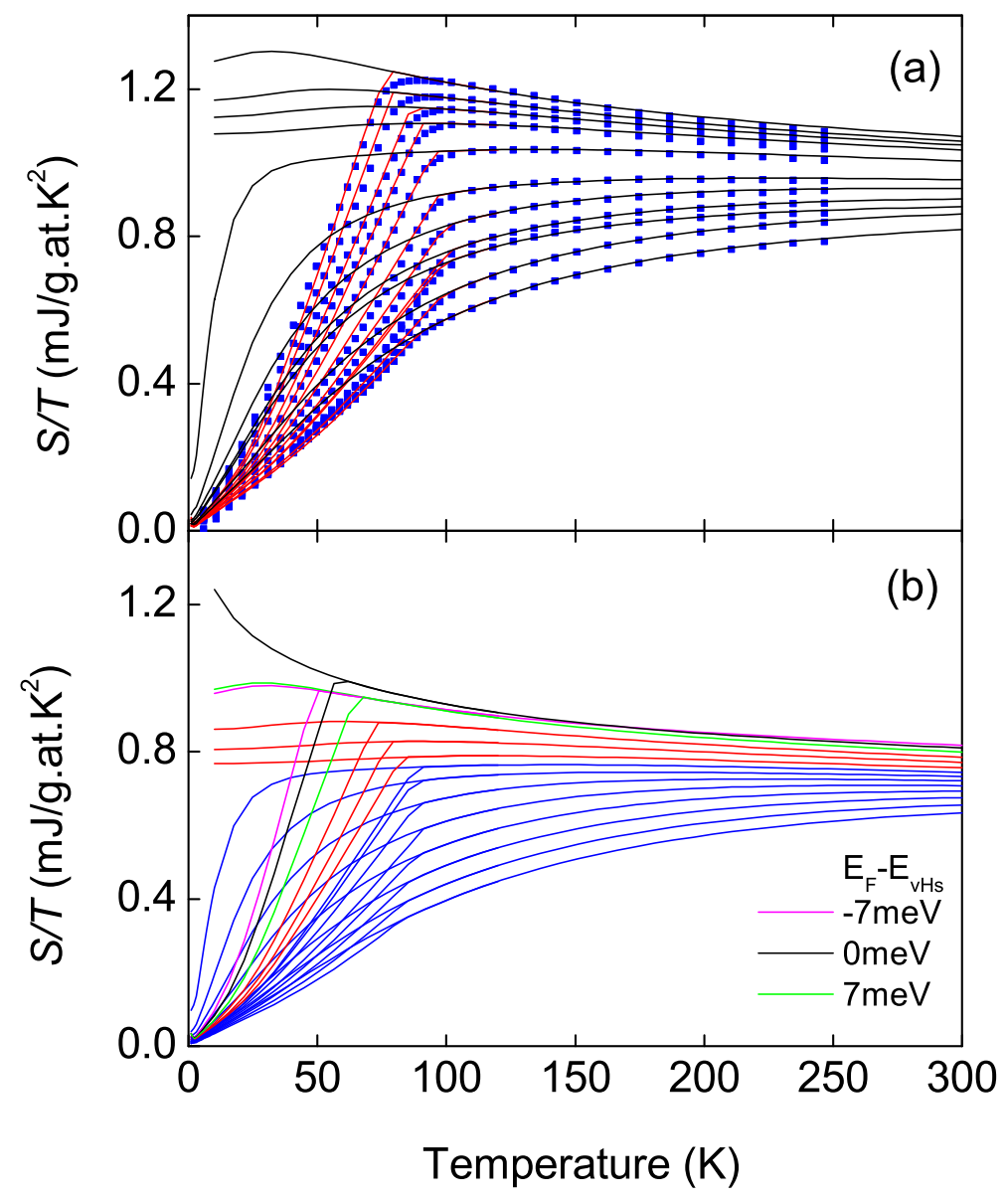

Figure 6.6: (a) Refined normal-state (black) and SC-state (red) fits to the electronic entropy data of Loram et al[122] for Bi-2212. For clarity every 20th data point only is shown. Each curve represents a different doping level from $p=0.129$ to 0.209 . (b) Unrefined absolute entropy curves with no fitting parameter. 
$S / T$ as temperature decreases.

The deduced values of $E_{F}-E_{v H s}$ and $E_{g}$ are plotted as a function of doping in Fig. 6.7(a) along with the measured $T_{c}$. The doping level has been determined from the empirical relation[18] $p=0.16 \pm 0.11 \sqrt{1-T_{c} / T_{c, \max }}$. The vHs crossing is projected to occur at $p=0.22$ exactly consistent with the ARPES data of Kaminski et al. (who observe a change in Fermi surface topology at $p=0.225)$, thus indirectly confirming their data and the ARPES technique in general. Good agreement between the $E_{F}-E_{v H s}$ values obtained from the entropy fits and those obtained from ARPES is found across the phase diagram as shown in Fig. 6.8. In view of the fact that ARPES is a surface technique dominated by the outermost $\mathrm{CuO}_{2}$ layer while the specific heat is a bulk property our conclusions effectively confirm both techniques. This is not a trivial conclusion. Both the fitted and measured values of $E_{F}-E_{v H s}$ differ somewhat from the expected doping dependence determined by integrating the density of states (shown by the dotted line in Fig. 6.8). However, the doping dependence calculated in this fashion depends on the accuracy of the tight binding fits at energies far below $E_{F}$, and the deviation may simply arise as a result of the rigid band approximation.

The remarkable success, illustrated in Fig. 6.6, in reconciling the bulk thermodynamics with the quasi-two-dimensional in-plane surface dynamics probed by ARPES shows just how much the $\mathrm{CuO}_{2}$ planes are decoupled. Pairing models based on inter-layer coupling[128] would appear to be heavily compromised by such results. This question is, of course, pertinent to the $\lambda_{c}$ isotope effect data discussed in Section 4.4.

We are now in a position to understand the progressive increase in entropy 


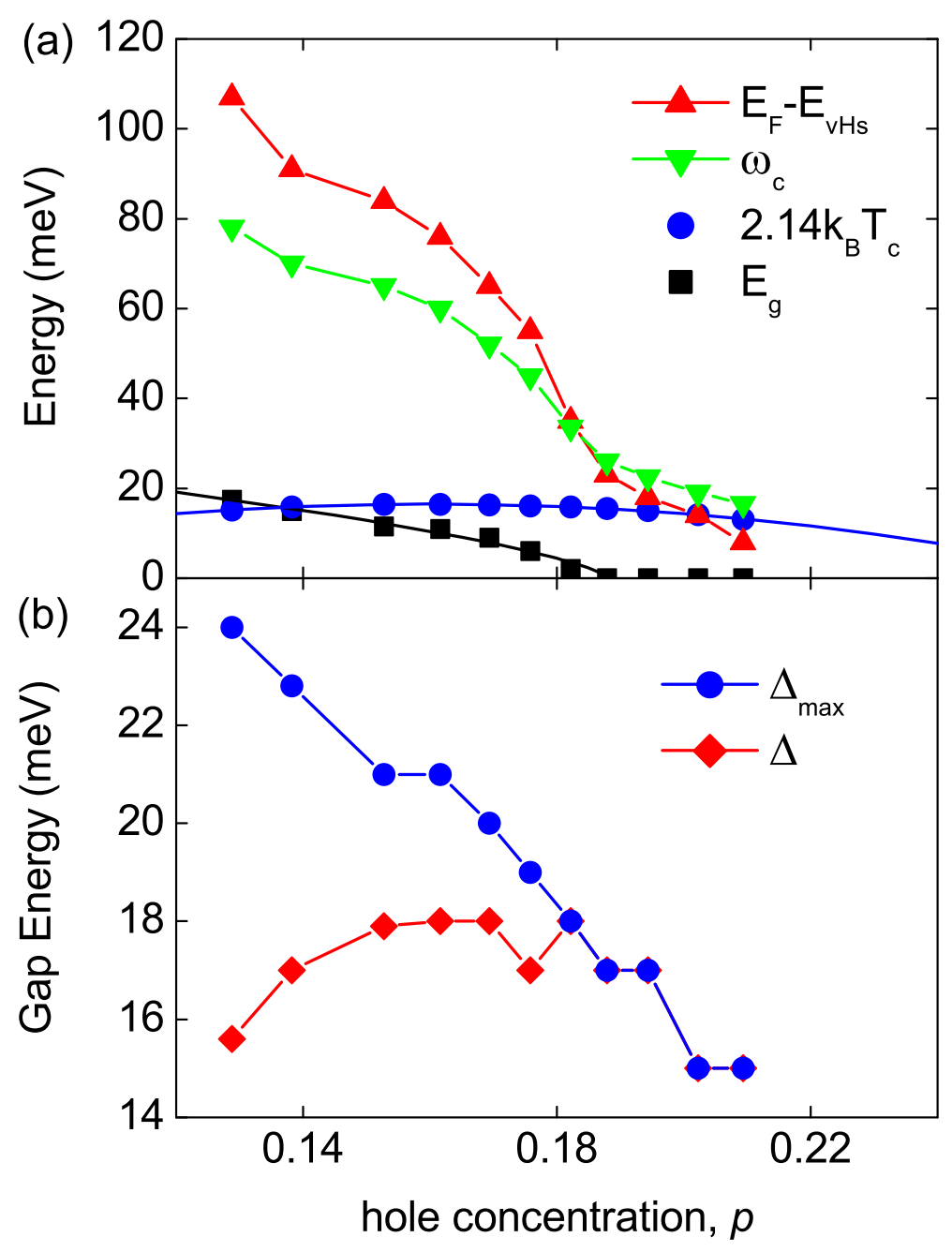

Figure 6.7: (a) $E_{F}-E_{v H s}$ (up triangles). The data suggests that the vHs will cross $E_{F}$ near $p=0.22$. Pairing potential energy cut-off $\omega_{c}$ (down triangles). Pseudogap magnitude $E_{g}$ (squares) and a fit given by Eqn. 6.8. The measured $T_{c}$ multiplied by $2.14 k_{B}$ (circles). (b) Maximum gap as measured from the DOS at $10 \mathrm{~K}$ (circles). $\mathrm{SC}$ gap measured from the DOS at $10 \mathrm{~K}$ in the absence of a pseudogap (diamonds). 


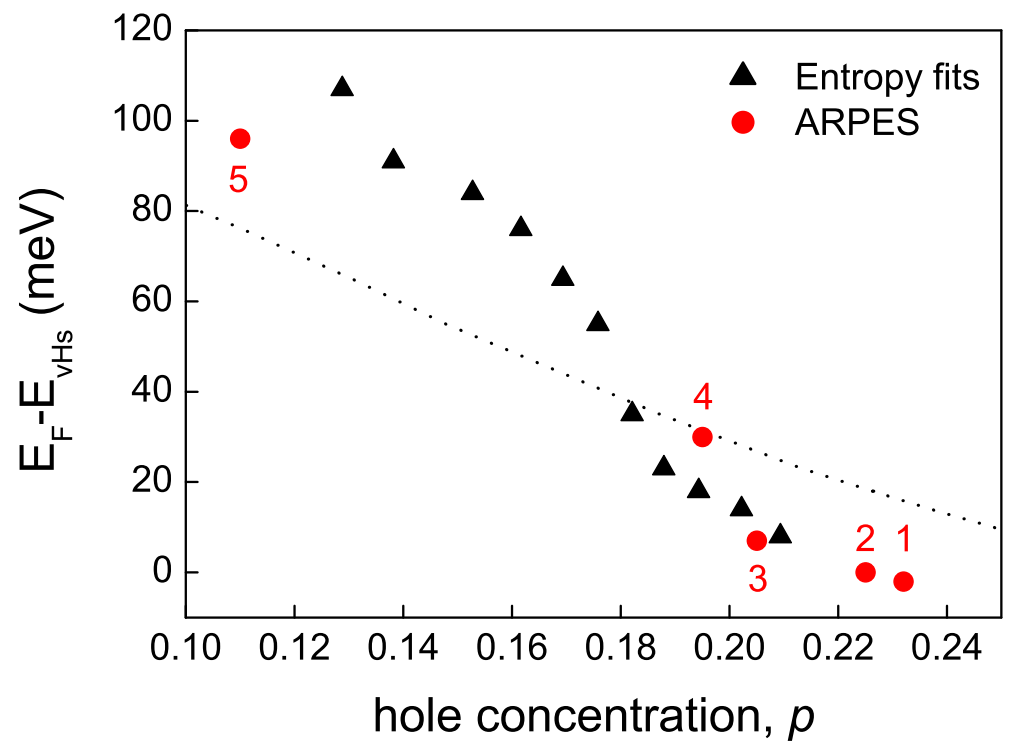

Figure 6.8: $E_{F}-E_{v H s}$ obtained from fits to the entropy (triangles) and from ARPES (circles). Points 1,2 and 3 are from Ref. [42], point 4 is from Ref. [40] and point 5 is from Ref. [126]. The dotted line shows the expected doping dependence of the Fermi energy, determined by integrating the density of states. 


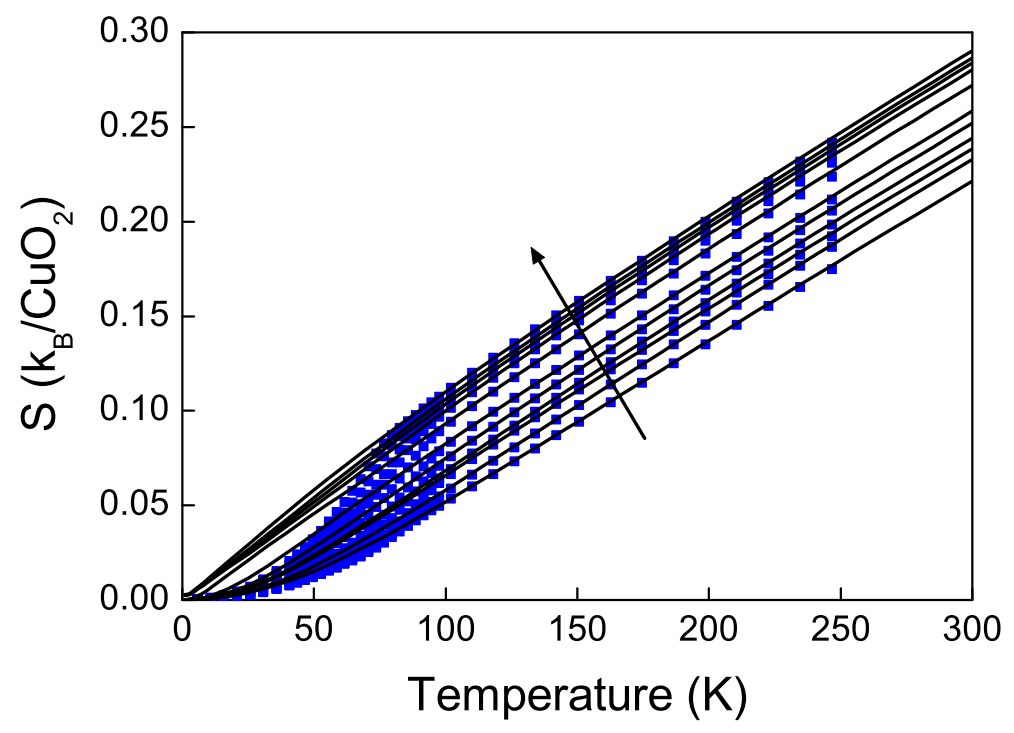

Figure 6.9: Electronic entropy vs temperature for different doping levels. For clarity every 20th data point only is shown. The lines are normal-state fits. The arrow indicates increasing doping.

with doping that has previously been estimated to be the classical value of one $k_{B}$ per added hole. Figure 6.9 shows Fig. 6.6(a) replotted as $S$ vs $T$ for different doping levels while Fig. 6.10 shows the same data plotted as $S$ vs $p$ for different temperatures. The latter plot reveals an average increment in entropy of about $0.85 k_{B}$ per added hole. For a $2 \mathrm{D}$ system of nearly free electrons this should be zero, and its near classical value was a point of significant interest to Loram et al.[122] We now see that this is just a signature of the approach to the $\mathrm{vHs}$ and the associated increase in DOS and spectral weight.

$S / T$ at fixed temperature rises with doping and reaches its maximum at the vHs crossing. Of course beyond the vHs $S$ vs $p$ is a decreasing function of doping. The maximum in $S / T$ is also observed in $\mathrm{La}_{2-x} \mathrm{Sr}_{x} \mathrm{CuO}_{4}[129]$ and 


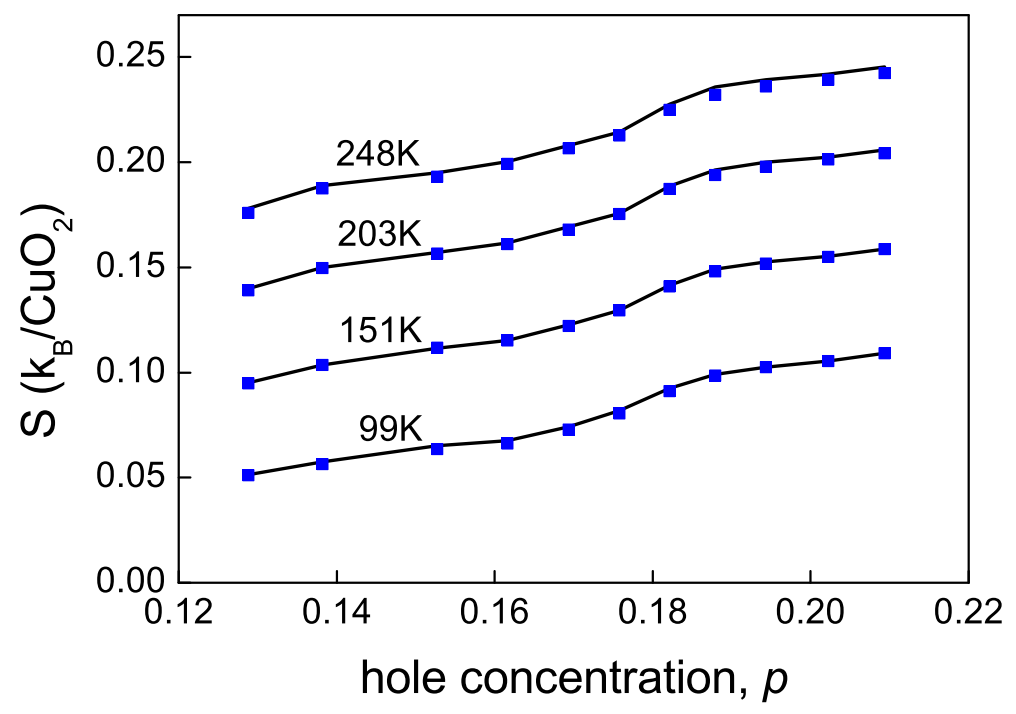

Figure 6.10: Electronic entropy vs doping at different temperatures. The lines are normal-state fits.

fits made to the data using (in the absence of suitable data for LSCO) an ARPES derived Bi-2201 single layer dispersion indicate a crossing between $p=0.24$ and 0.27 (see Fig. 6.11).

In their paper Loram et al.[129] explain the entropy maximum as being due to a peak in the DOS at the Fermi level that grows and collapses as a function of doping. They dismiss the possibility of a vHs passing through the $E_{F}$ with doping, arguing that the temperature dependence of the chemical potential will prevent the large changes observed in entropy as the doping is changed. The fits made to the Bi-2212 and LSCO data show that this is not the case. The vHs does indeed result in a strongly temperature dependent chemical potential. However the amount by which it changes with temperature depends on the height and extent of the vHs with respect to the background density of states. 


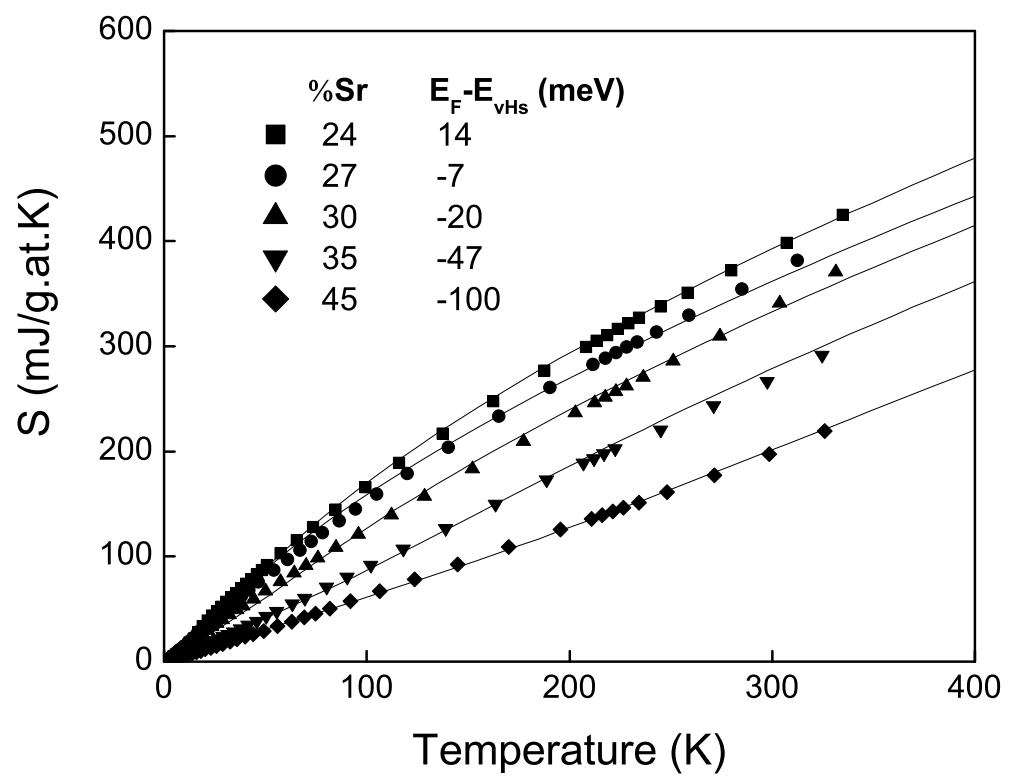

Figure 6.11: Fits to overdoped LSCO entropy data[129] using an ARPES derived Bi-2201 dispersion. For clarity every 10th data point only is shown. The Fermi level crosses the vHs between $p=0.24$ and 0.27 . 
The pseudogap is observed to open at critical doping $p_{\text {crit }}=0.188$ in agreement with previous analyses[122, 81]. $E_{g}$ has been fitted with the following equation

$$
T^{*}(p)=E_{g} / k_{B}=T_{0}^{*}\left(1-p / p_{\text {crit }}\right)^{1-\alpha}
$$

with $T_{0}^{*}=443.7 \mathrm{~K}$ and $\alpha=0.317$. These values agree with the results of Naqib et al. [130] who have determined $T^{*}(p)$ of $\mathrm{Y}_{1-x} \mathrm{Ca}_{x} \mathrm{Ba}_{2}\left(\mathrm{Cu}_{1-y} \mathrm{Zn}_{y}\right) \mathrm{O}_{7-\delta}$ from transport measurements. A fit to their data gives $T_{0}^{*}=510 \mathrm{~K}$ and $\alpha=0.2$. The sublinear behaviour of $T^{*}(p)$ and non-zero value of $\alpha$ is expected if $p_{\text {crit }}$ is a quantum critical point[131].

From the SC state fits the energy cut-off, $\omega_{c}$, is surprisingly found to be linearly related to the distance of the Fermi level from the vHs, see Fig. 6.12. In particular $\omega_{c}(m e V)=10.96+0.637\left(E_{F}-E_{v H s}\right)$ with correlation coefficient $R=0.99945$. Because of this rapid fall in energy scale with doping this behaviour is suggestive of spin-fluctuation mediated superconductivity or perhaps electron-phonon coupling enhanced by spin-fluctuations, but probably not coupling to phonons alone.

Fig. 6.7(b) shows the magnitude of the combined SC gap and pseudogap, $\Delta_{\max }$, measured from the calculated DOS at $10 \mathrm{~K} . \Delta_{\max }$ increases with decreasing doping just as observed from ARPES[132], tunnelling[133] and Raman scattering[134]. Also plotted is the $\mathrm{SC}$ gap magnitude $\Delta$, determined by setting $E_{g}=0$ and measuring the gap in the calculated DOS at 10K. The magnitude is smaller than typically observed because of the weak coupling assumption for which $2 \Delta / k_{B} T_{c}=4.28$. The gap, $\Delta$, rises and falls 


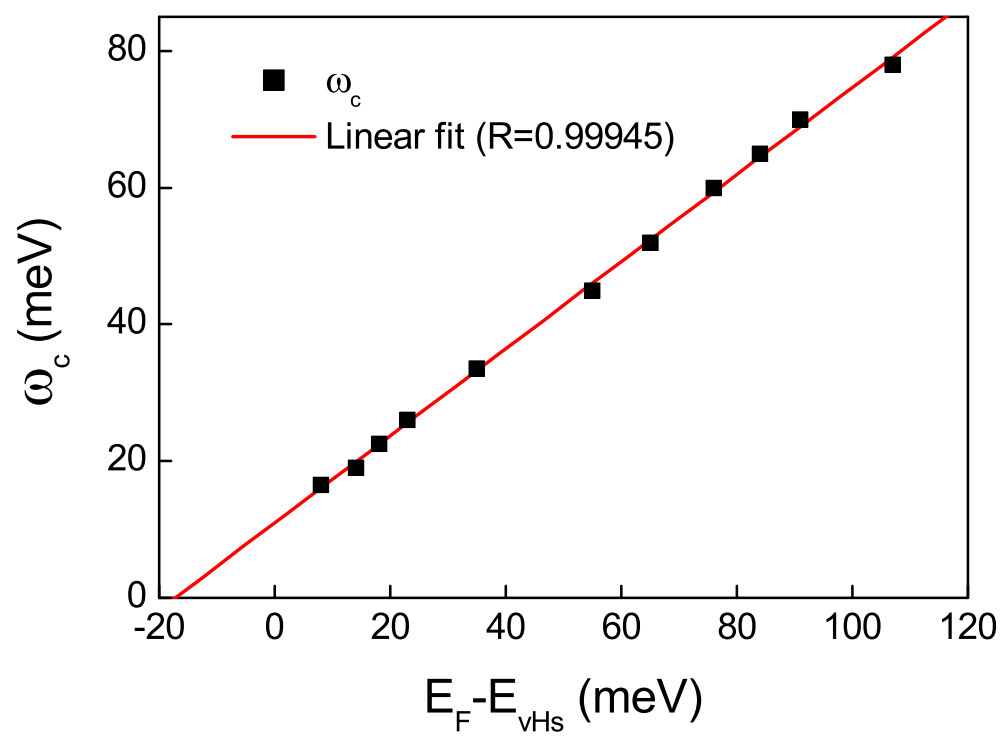

Figure 6.12: Pairing potential cut-off energy vs distance from antibonding van Hove singularity.

in conjunction with the observed $T_{c}$. It is important to note that the experimentally observed monotonic increase in the gap magnitude with decreasing doping is here seen to be associated with the pseudogap, and not the SC gap as generally believed. The behaviour here is consistent with the twogap picture presented by Deutscher[76] and more recently by Le Tacon et al.[77] but has been a feature of the work of Loram and Tallon for a long time[127, 67, 81]. 


\subsection{Superfluid Density}

The superfluid density, $\rho_{s}$, is proportional to the inverse square of the penetration depth given by[135]

$$
\begin{aligned}
\frac{1}{\lambda_{a b}^{2}} & =\frac{\mu_{0} e^{2} n}{4 \pi \hbar^{2}} \sum_{\mathbf{k}}\left[\left(\frac{\partial \epsilon(\mathbf{k})}{\partial k_{x}}\right)^{2} \frac{\Delta^{2}(\mathbf{k})}{E^{2}(\mathbf{k})}-\frac{\partial \epsilon(\mathbf{k})}{\partial k_{x}} \frac{\partial \Delta(\mathbf{k})}{\partial k_{x}} \frac{\Delta(\mathbf{k}) \epsilon(\mathbf{k})}{E^{2}(\mathbf{k})}\right] \\
& \times\left[\frac{1}{E(\mathbf{k})}-\frac{\partial}{\partial E(\mathbf{k})}\right] \tanh \left(\frac{E(\mathbf{k})}{2 k_{B} T}\right)
\end{aligned}
$$

where $n$ is the electron volume density. This equation has the desirable property of explicitly yielding $\rho_{s}=0$ in the normal limit, $\Delta\left(\mathbf{k}, T=T_{c}\right)=0$, even in the presence of a normal-state pseudogap. This statement is made simply because other forms have been proposed which do not exhibit this property (see for example Ref. [136]). The summation is performed over both the bonding and anti-bonding bands and $\Delta$ is assumed to be the same for both bands[125].

Using the parameters obtained from the entropy fits in section 6.1 the superfluid density was calculated using Eqn. 6.9 with no further adjustable parameters. The temperature dependence is shown in Fig. 6.13(a). For comparison (and in the absence of data for Bi-2212) Fig. 6.13(b) shows the $a b$-plane superfluid density of $\mathrm{La}_{2-x} \mathrm{Sr}_{x} \mathrm{CuO}_{4}$ determined by ac-susceptibility measurements on grain-aligned samples reported by Panagopoulos et al.[137]. There is excellent qualitative and quantitative agreement between the calculated and observed results.

Panagopoulos et al. speculated that the significant deviation of the $\mathrm{x}=0.24$ data from the classic flat band $d$-wave temperature dependence re- 
flected a change in electronic structure connected with the transition from a hole-like to electron-like Fermi surface near $\mathrm{x}=0.27$. The calculations confirm that the increasing linearity of $\rho_{s}(T)$ with overdoping can now be understood in terms of the approach to the vHs where full linearity occurs. (The crossing of the vHs in LSCO can also be inferred from the systematic rise then fall observed in the entropy near $p=0.24[129]$. See also Fig. 6.11.)

The opening of the pseudogap leads to the strong reduction in $\rho_{s}$ observed below $p=0.19$, a result found previously by Tallon et al[138]. This is clearly illustrated by the plot of $\rho_{s}(10 \mathrm{~K})$ vs $p$ in Fig. 6.14. The overall doping dependence and absolute magnitude of $\rho_{s}(10 \mathrm{~K})$ concurs almost exactly with experimental data for Bi-2212[139] also shown in Fig. 6.14. We recall that no fitting parameters are used in Eqn. 6.9. It is remarkable that $S / T$ and $\rho_{s}(T)$ are so similar in LSCO, Bi-2212 and indeed $\mathrm{Y}_{1-x} \mathrm{Ca}_{x} \mathrm{Ba}_{2} \mathrm{Cu}_{3} \mathrm{O}_{7-\delta}$, despite the significant differences in bare band structure. It is a central conclusion to this work that, despite wide variations in bare band structure, the renormalized dispersion near $E_{F}$ seems to lead to a universal phenomenology seen here in the superfluid density, but also in the entropy, the susceptibility, the thermoelectric power and the universal nodal Fermi velocity[98]. This observation calls for theoretical explanation within a strong-coupling picture.

In the underdoped data the downturn seen at low $T$ and $p$ in the calculated $\rho_{s}(T)$ curves arises from the closing of the Fermi arcs and is not observed in the experimental data which shows an upturn at low $T$ and $p$. A similar downturn would occur in the condensation energy, which again is not observed. This indicates that the Fermi arc picture is, at best, incomplete. 


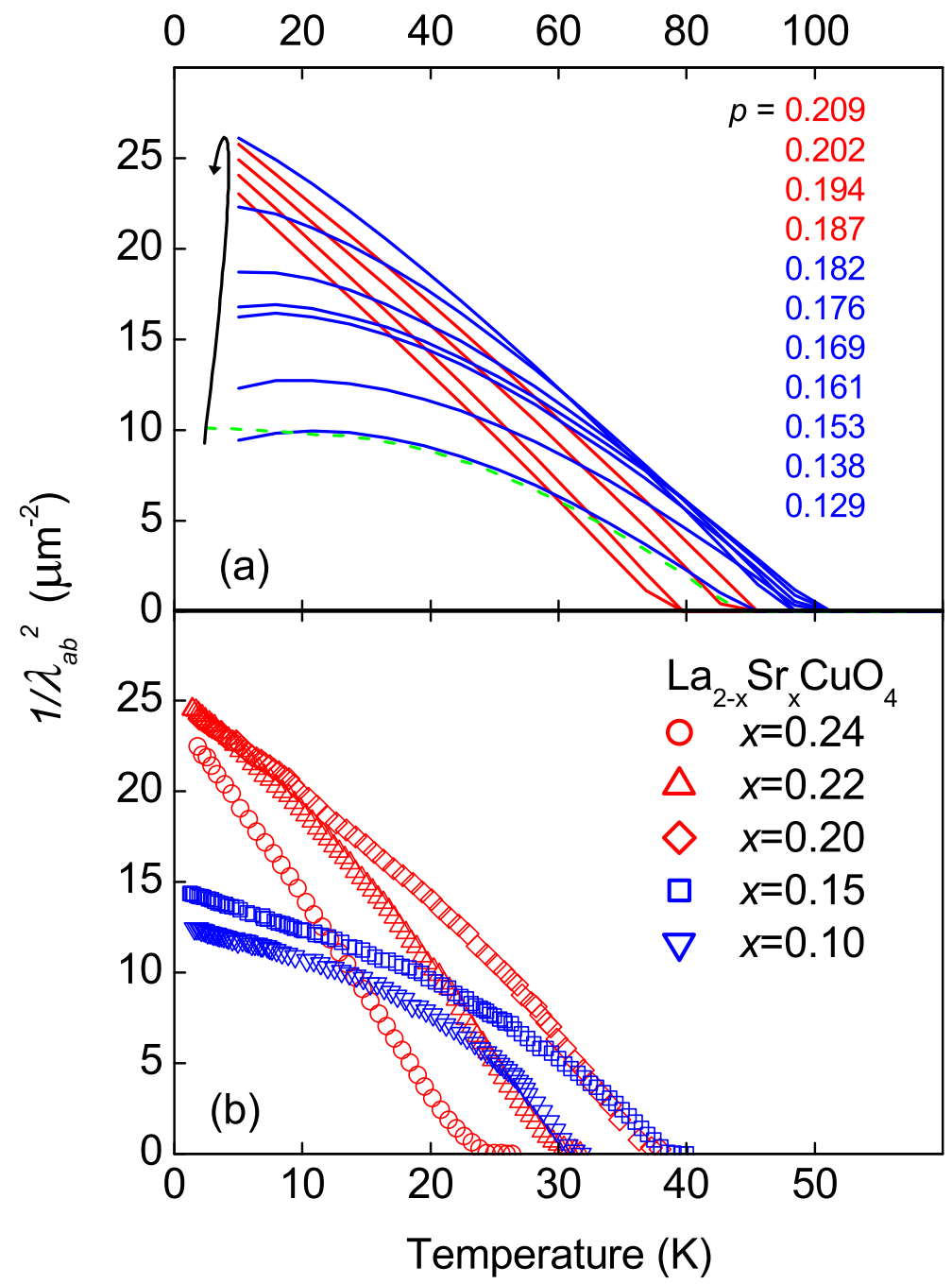

Figure 6.13: (a) Superfluid density computed using parameters from the entropy fits in Fig. 6.6. The arrow points in the direction of increasing doping. Curves/data in which the pseudogap is present are shown in blue. Linearly reducing $E_{g}$ by ten percent between $20 \mathrm{~K}$ and $0 \mathrm{~K}$ results in the green dotted curve. (b) Superfluid density of LSCO obtained from ac-susceptibility measurements [137] 


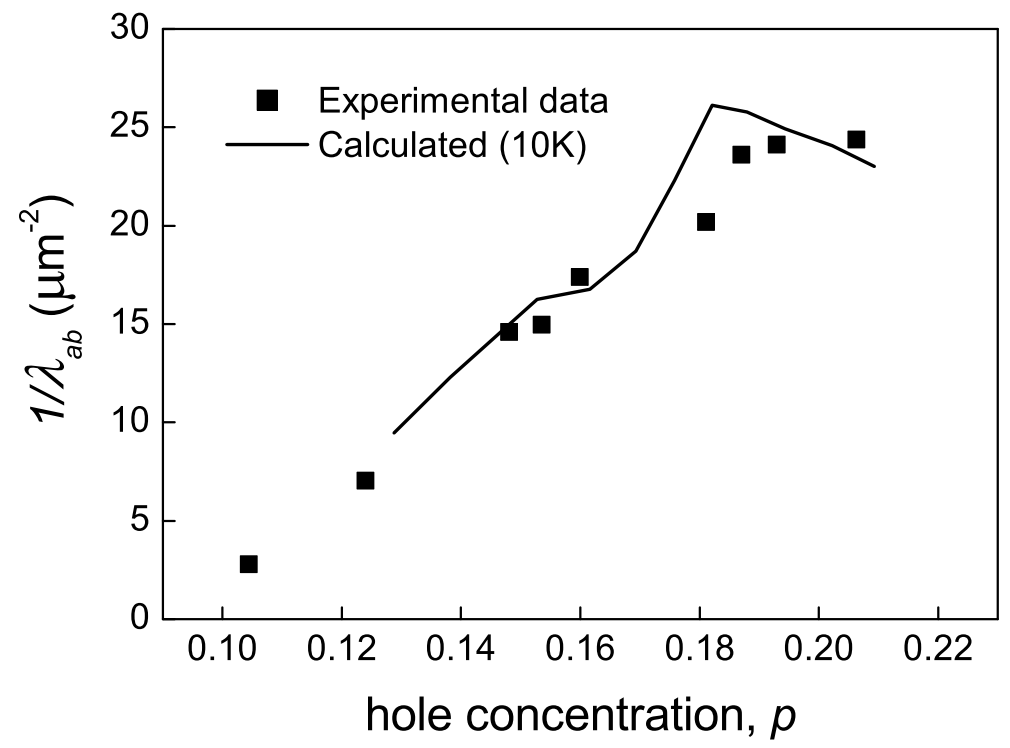

Figure 6.14: Comparison of the calculated superfluid density at $10 \mathrm{~K}$ with measured low-T Bi-2212 superfluid density data from Ref. [139] (squares).

The upturn may be due to a redistribution of spectral weight from the pseudogap to the superconducting gap at low temperatures. This explanation is supported by ARPES data[72] showing a decrease in the antinodal gap magnitude with a decrease in temperature, suggestive of a reduction in the pseudogap magnitude $E_{g}$. Linearly reducing $E_{g}$ by ten percent between $20 \mathrm{~K}$ and $0 \mathrm{~K}$ results in the green dotted curve plotted in Fig. 6.13(a). Alternatively, the Fermi arc picture is as we say incomplete or perhaps even faulted. This issue is examined in more detail in the following section.

\subsection{Raman Response}

Raman scattering allows a means to probe the Fermi arcs at low temperature.

To calculate the Raman response we employ the single band six parameter 
tight-binding Bi-2212 dispersion, $\epsilon(\mathbf{k})$, reported by Norman et al.[32] and assume a rigid-band approximation. Inclusion of bilayer splitting will not significantly affect the following results. We take the Fermi level to be $34 \mathrm{meV}$ above the van Hove singularity at $(\pi, 0)$ near optimal doping[32], $96 \mathrm{meV}$ above the vHs in an underdoped sample with doping $p=0.11[126]$, and interpolate in between for intermediate doping levels. The imaginary part of the unscreened non-resonant Raman response at $T=0$ is proportional to $[140]$

$$
\chi_{0}^{\prime \prime}(q=0, \omega)=\int \frac{d^{2} k}{(2 \pi)^{2}} \delta[\omega-2 E(\mathbf{k})] \frac{|\Delta(\mathbf{k})|^{2}}{E^{2}(\mathbf{k})}|\gamma(\mathbf{k})|^{2}
$$

where the integral is over occupied states below $E_{F}$,

$\Delta(\mathbf{k})=\frac{1}{2} \Delta_{0}\left(\cos k_{x}-\cos k_{y}\right)$ is the $d$-wave symmetric superconducting gap function and $E(\mathbf{k})=\sqrt{\epsilon^{2}(\mathbf{k})+|\Delta(\mathbf{k})|^{2}}$. The Raman vertex functions,

$\gamma(\mathbf{k})^{B_{1 g}}=\gamma B_{1 g}\left(\cos k_{x}-\cos k_{y}\right)$ and $\gamma(\mathbf{k})^{B_{2 g}}=\gamma B_{2 g} \sin k_{x} \sin k_{y}$, respectively probe the antinodal and nodal regions of the dispersion as illustrated in Fig. 6.15. The magnitude of the superconducting gap, $\Delta_{0}$, is taken from the weak-coupling $d$-wave result $2 \Delta_{0}=4.28 k_{B} T_{c}$ with $T_{c}$ given by the empirical relation[18] $T_{c} / T_{c, \max }=1-82.6(p-0.16)^{2}$. We adopt the model pseudogap given by Eqn. 6.4 but, in order to reflect the Kanigel results, we replace $\theta_{0}$ by

$$
\theta_{0}=\frac{\pi}{4}\left(1-\frac{T}{T^{*}}\right) \quad\left(T<T^{*}\right)
$$

where $T^{*}=E_{g, \max } / k_{B}$. This results in a pseudogap that fills linearly with temperature up to $T^{*}$ where it fills completely. As in section 6.1 the pseudogap is implemented as a states-non-conserving gap by eliminating states with $\epsilon(\mathbf{k})<E_{g}$ from the integration in equation 6.10. The doping depen- 

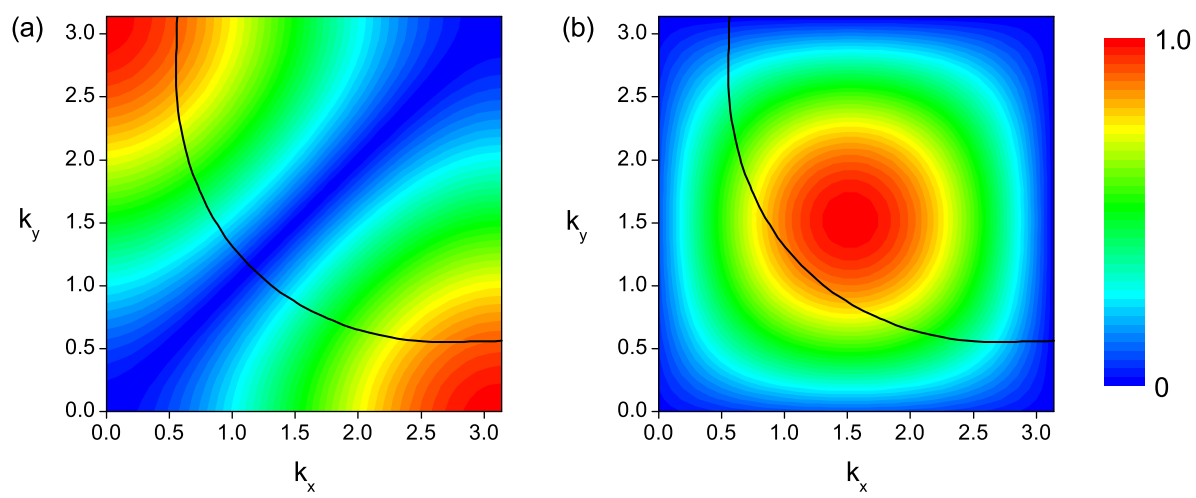

Figure 6.15: Different regions of the Fermi surface (black curve) can by probed by Raman spectroscopy. Normalized magnitude of (a) the $B_{1 g}$ Raman vertex $\gamma(\mathbf{k})^{B_{1 g}}=\gamma B_{1 g}\left(\cos k_{x}-\cos k_{y}\right)$, and (b) the $B_{2 g}$ Raman vertex, $\gamma(\mathbf{k})^{B_{2 g}}=$ $\gamma B_{2 g} \sin k_{x} \sin k_{y}$.

dence of $E_{g}$ is obtained from measurements of the leading-edge ARPES gap at $100 \mathrm{~K}[127]$.

Figure 6.16(b) shows the antinodal $\left(B_{1 g}\right)$ Raman response for six hole concentrations spanning the range 0.12 to 0.19 . Here we have assumed that the length of the Fermi arc becomes fixed at the onset of superconductivity, implemented by setting $T=T_{c}$ in equation 6.11. Figure 6.16(a) shows the nodal $\left(B_{2 g}\right)$ response. The calculations closely resemble the recently reported Raman scattering results of Le Tacon et al.[77] which are reproduced in Fig. 6.17. The pseudogap peak maximum in the $B_{1 g}$ response shifts monotonically to higher energies with decreasing doping. Simultaneously the intensity of this peak rapidly reduces with underdoping. In contrast, the superconducting peak maximum in the $B_{2 g}$ response is found to shift to lower energies in the underdoped regime. The magnitude of the nodal peak persists down to the lowest doping levels. Also reproduced is the increased 


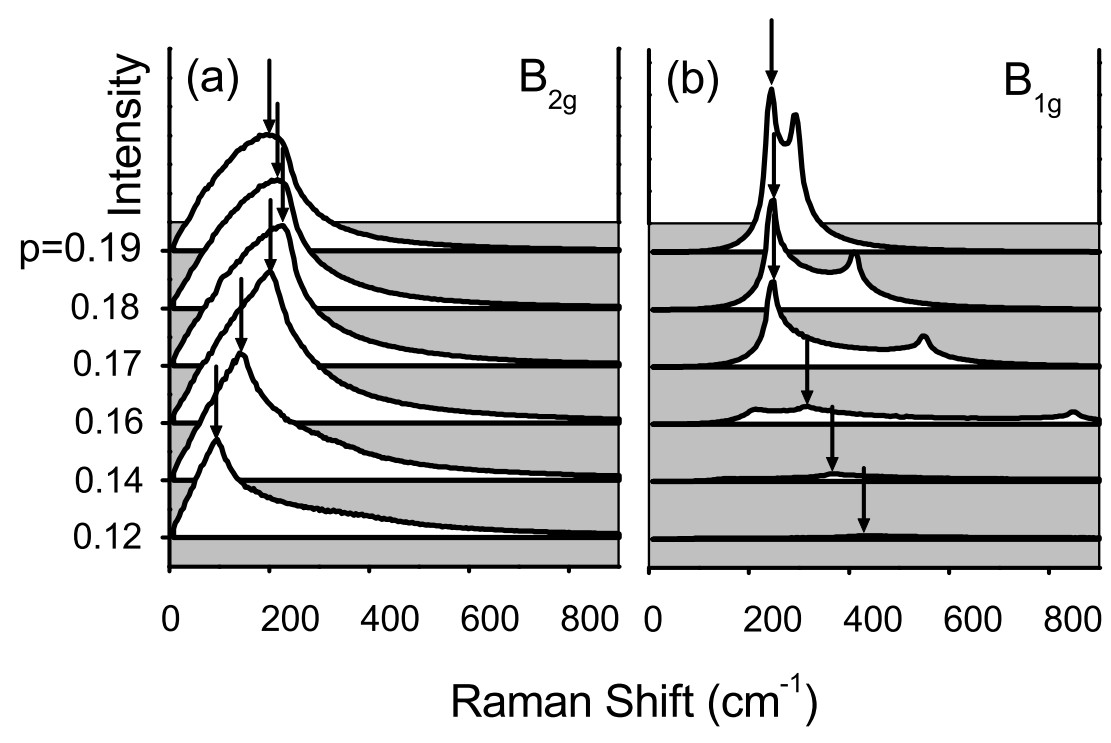

Figure 6.16: Calculated Raman response at $T=0$ for a Fermi arc length fixed below $T_{c}$ : (a) nodal $B_{2 g}$ and (b) antinodal $B_{1 g}$ symmetry. Arrows show the positions of superconducting gap and pseudogap features.

linear slope of the response at low dopings.

Figure 6.18 shows the nodal response in the alternative case where we have assumed that the Fermi arcs continue to collapse in the superconducting state. This has been implemented by setting $T=0$ in equation 6.11 resulting in a fully nodal pseudogap. In this case the $B_{2 g}$ peak shifts monotonically to higher energies with decreasing doping and the intensity reduces rapidly. This behaviour is not observed experimentally.

In light of these calculations, the Raman data implies the existence of a finite Fermi arc in the normal state zero temperature limit (or something else equivalent to this). The observed changing shape of the gap from a ' $\mathrm{V}$ shape' to a 'U-shape'[30, 125] as doping is reduced follows naturally from this scenario (see Fig. 6.19). With temperature dependent Raman measurements 


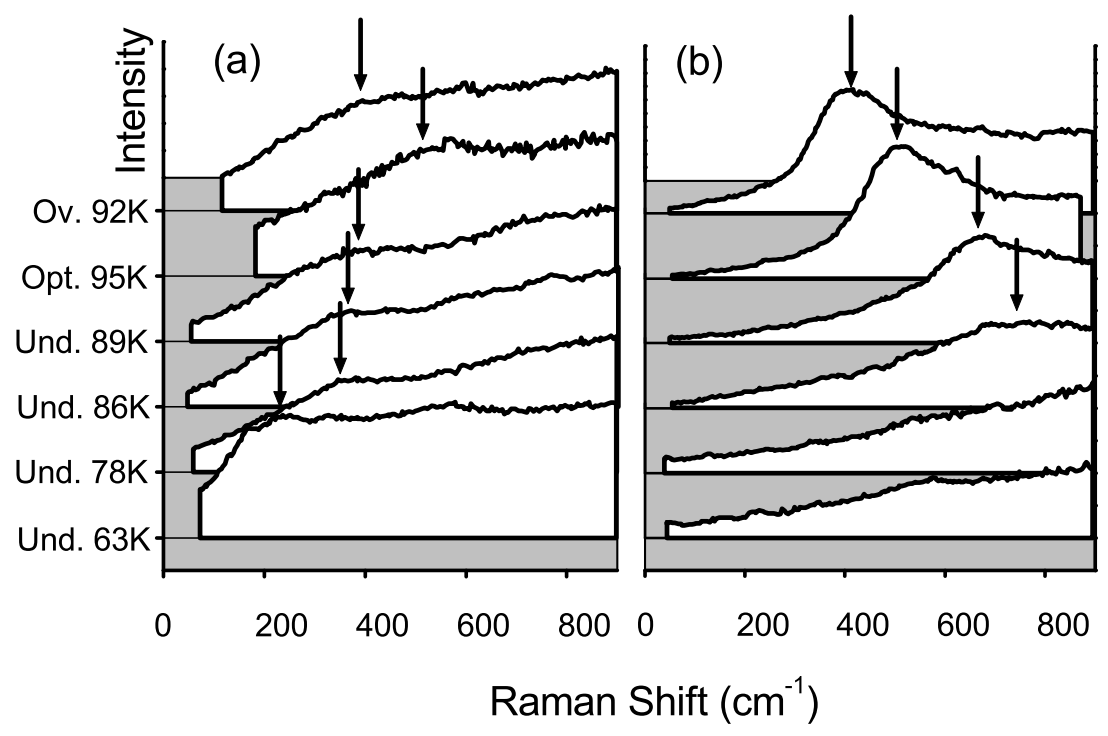

Figure 6.17: Experimental Raman data for $\mathrm{HgBa}_{2} \mathrm{CuO}_{4+\delta}$ from Le Tacon et al[77]. (a) $B_{2 g}$ and (b) $B_{1 g}$ Raman response $T \ll T_{c}$.

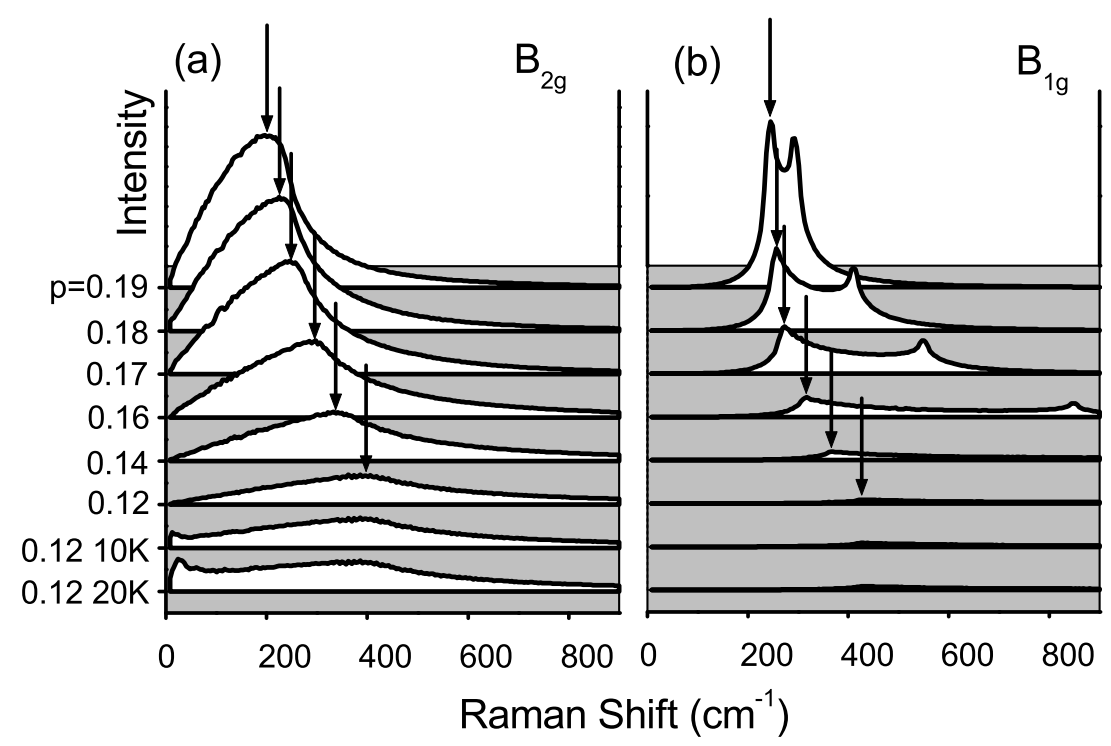

Figure 6.18: Calculated (a) nodal $B_{2 g}$ and (b) antinodal $B_{1 g}$ Raman response for a fully nodal pseudogap at $T=0$. Also shown is the Raman response at $10 \mathrm{~K}$ and $20 \mathrm{~K}$ for the $p=0.12$ doping state. The growing peak at low frequency is due to the growth of the Fermi arcs. The intensity scale is the same for all plots. 


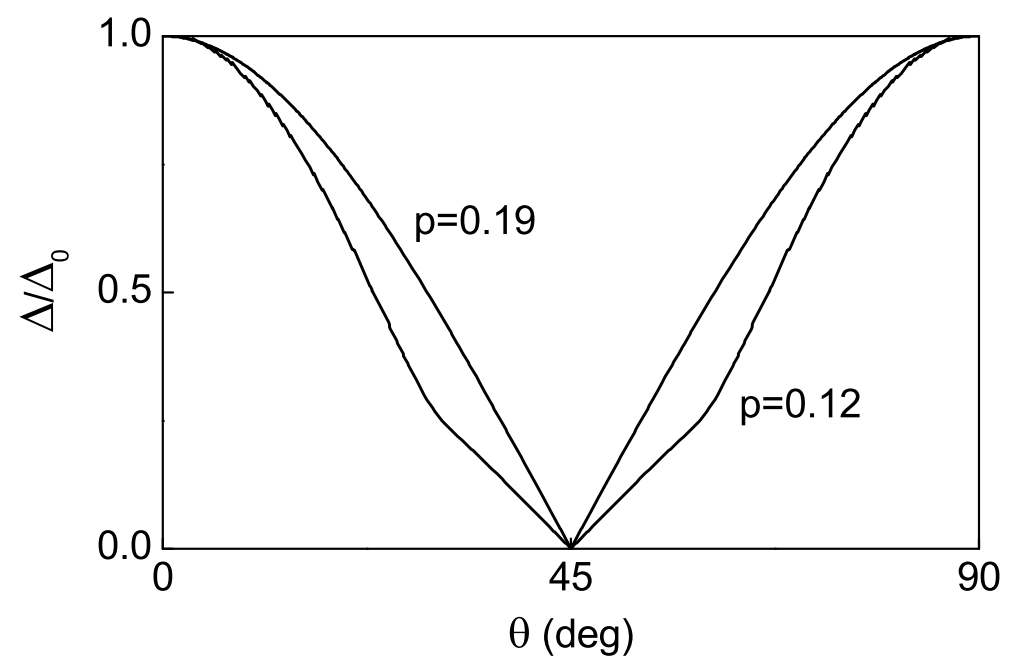

Figure 6.19: Gap shape for $\mathrm{p}=0.19$ and $\mathrm{p}=0.12$ as a function of Fermi surface angle. In underdoped samples the gap is small near the nodal region, arising from the superconducting gap opening on the Fermi arcs.

it may be possible to determine whether the Fermi arcs freeze at $T_{c}$ or instead continue to shrink to a finite extent at $T=0$. The two lower-most curves in Fig 6.18 show the $B_{2 g}$ response calculated at $10 \mathrm{~K}$ and $20 \mathrm{~K}$ for the $p=0.12$ doping state. A peak appearing at low frequency, which shifts to higher frequencies with increasing temperature, is a signature of the growing Fermi arcs. These curves are calculated under the assumption that corrections to the Raman response equation (6.10) for temperature are negligible. To confirm this effect will require careful measurements of the low-frequency $B_{2 g}$ response of a heavily underdoped sample at a number of temperatures well below $T_{c}$.

In section 6.1 it was shown that the fully nodal pseudogap could not be included in the self-consistent calculation of the superconducting gap because it removed too many of the low-energy states that contribute the most weight 
in the BCS gap equation (Eqn. 6.6). A finite Fermi arc at zero temperature results in a finite density of states at the Fermi level. And so it is now possible to explore the effect of including the pseudogap in the BCS gap equation. The entropy is recalculated as in section 6.1 but with the $\pi / 4$ in Eqn. 6.5 replaced with an adjustable parameter $\theta_{\text {max }}$. To offset the effect of the finite density of states at the Fermi level the fill rate is slowed from $\left[1-\tanh \left(T / T^{*}\right)\right]$ to $\left[1-\tanh \left(T / 2 T^{*}\right)\right]$.

Figure 6.20(a) shows normal- and superconducting-state fits to the most underdoped entropy/ $T$ dataset of Loram et al.[122] where the Fermi arcs shrink down to a finite length $\left(\theta_{\max }=35 \mathrm{deg}\right)$ at zero temperature. The pseudogap is included in the self-consistent calculation of $\Delta$, which is plotted in Fig. 6.20(b) along with the resulting superfluid density. $\Delta$ exhibits a downturn at low temperatures while the superfluid density exhibits an upturn. Under this scheme the Fermi level is $93 \mathrm{meV}$ from the vHs, slightly closer than $107 \mathrm{meV}$ in the case of a fully nodal non-self-consistent pseudogap. This only improves the agreement with the $E_{F}-E_{v H s}$ values obtained from ARPES (see Fig. 6.8). The pseudogap magnitude is nearly unchanged at $16.5 \mathrm{meV}$ compared with $17.5 \mathrm{meV}$. In order to accommodate the pseudogap either the pairing potential amplitude, $\mathrm{V}$, or the energy cut-off, $\omega_{c}$ must be increased.

Note that if a completely temperature independent pseudogap is assumed then the Fermi level gets pushed much closer to the vHs and the disagreement with the ARPES values becomes significant. A further consequence is that the observed decrease in superfluid density is not observed. These calculations usefully indicate the trends and could be refined in such a way to yield a more realistic temperature dependence of the gap and a less strong 

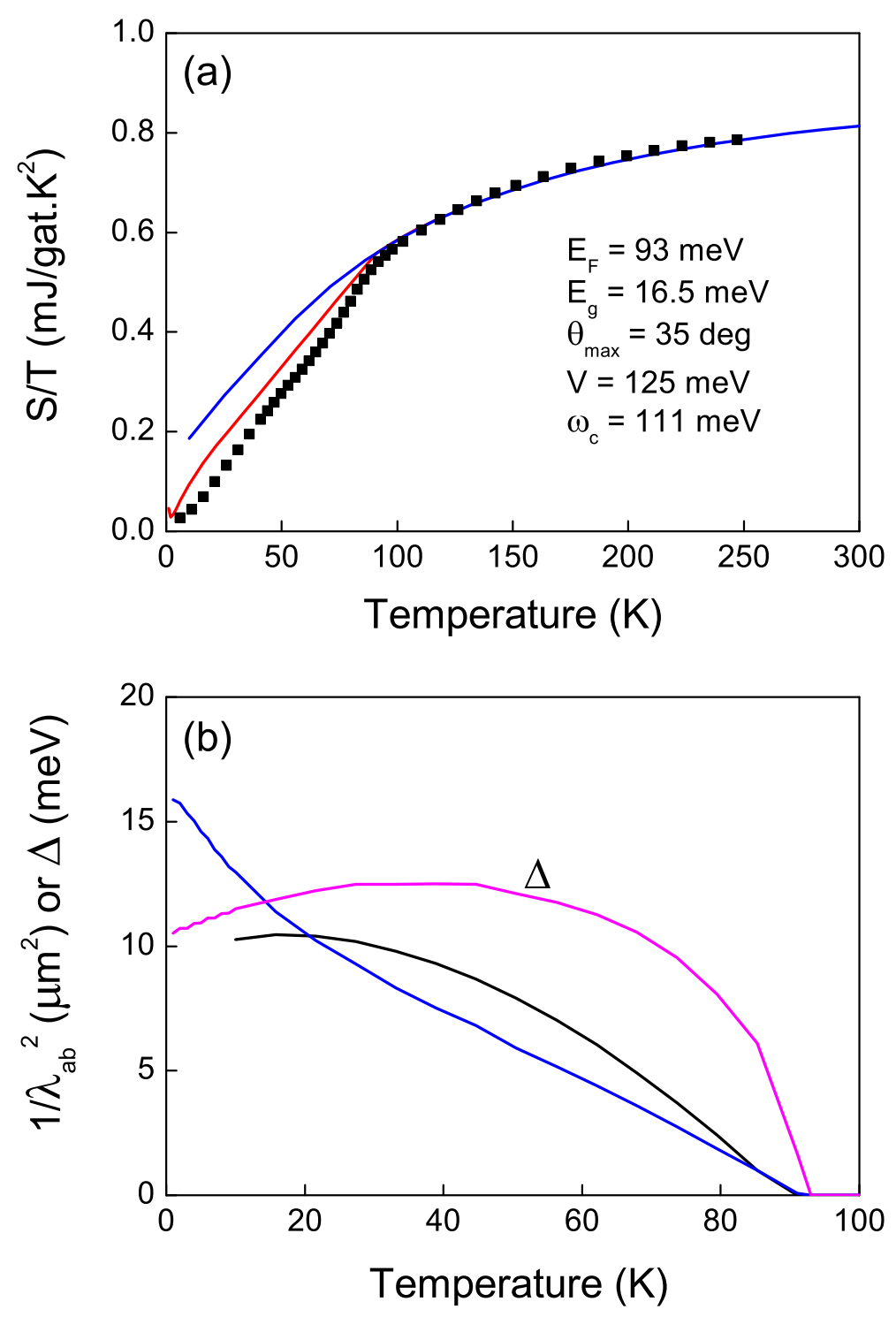

Figure 6.20: (a) Normal state (blue) and superconducting state (red) fits to the $p=0.129$ electronic entropy $/ T$ data of Loram et al. [122] where the Fermi arcs shrink to a finite length $\left(\theta_{\max }=35 \mathrm{deg}\right)$ at $T=0$. The pseudogap is included in the selfconsistent calculation of $\Delta$, shown in (b). Also shown is the resulting superfluid density (blue) compared with that calculated in section 6.1 in the presence of a fully nodal pseudogap (black). 
upturn in $\rho_{s}$ as $T \rightarrow 0$.

\subsection{Spin Susceptibility}

The spin susceptibility for a system of electrons in the static, long wavelength limit is given by[141]

$$
\begin{aligned}
\chi_{s} & =2 \mu_{B}^{2} \int N(E)\left(-\frac{\partial f}{\partial E}\right) d E \\
& =\frac{\mu_{B}^{2}}{2 k_{B} T} \int N(E) \operatorname{sech}^{2}\left(\frac{E-\mu}{2 k_{B} T}\right) d E
\end{aligned}
$$

where $N(E)$ is the density of states and $f$ is the Fermi function. The NMR Knight shift is related to the spin susceptibility by[141]

$$
K(T)=\alpha \chi_{s}(T)+K_{o r b}
$$

where $\alpha$ is the hyperfine coupling constant and $K_{\text {orb }}$ is the temperature independent orbital shift made up of the Van Vleck orbital susceptibility, $\chi_{V V}$, and the chemical shift, $\delta[142,143]$. In this section we fit the $\mathrm{Y}_{0.8} \mathrm{Ca}_{0.2} \mathrm{Ba}_{2} \mathrm{Cu}_{3} \mathrm{O}_{7-\delta}(\mathrm{Y}-123){ }^{89} \mathrm{Y}$ NMR Knight shift data of Williams et al.[144] using a rigid ARPES energy-momentum dispersion and the model pseudogap of section 6.1. The data spans a wide range of doping ( $p=0.136$ to 0.234 ) and the temperature and doping dependence closely resembles the behaviour of the Bi-2212 entropy/T data (see Fig. 6.6).

For Y-123 we employ a two-dimensional bilayer dispersion $\epsilon(\mathbf{k})$ provided by the authors of Ref. [145], which was obtained from tight binding fits to 
Table 6.2: Tight binding basis functions and coefficients (in $\mathrm{eV}$ ) for the bonding and antibonding bands of Y-123. The energy-momentum dispersion is given by $\epsilon(\mathbf{k})=\sum c_{i} \eta_{i}(\mathbf{k})$.

\begin{tabular}{|c|c|c|}
\hline$c_{i}$ (Bonding) & $c_{i}$ (Antibonding) & $\eta_{i}(\mathbf{k})$ \\
\hline 0.488 & 0.650 & 1 \\
-2.576 & -1.912 & $\frac{1}{2}\left(\cos k_{x}+\cos k_{y}\right)$ \\
1.080 & 0.700 & $\cos k_{x} \cos k_{y}$ \\
-0.312 & -0.1880 & $\frac{1}{2}\left(\cos 2 k_{x}+\cos 2 k_{y}\right)$ \\
\hline
\end{tabular}

high-resolution ARPES data. The tight binding basis functions and coefficients for the bonding and antibonding bands are listed in Table 6.2. The density of states is shown in Fig. 6.21. Comparison with the Bi-2212 density of states (Fig. 6.3) reveals that the anti-nodal bilayer splitting in Y-123 $(\approx 500 \mathrm{meV})$ is about five times larger than in Bi-2212 $(\approx 100 \mathrm{meV})$. Also, as seen in the inset to Fig. 6.21, the Fermi surface splitting is not restricted to the antinodal regions.

Fits to the NMR data are shown in Fig. 6.22(a) where a doping and temperature independent value for $K_{\text {orb }}$ of $235 \mathrm{ppm}$ has been used. An excellent match with the data is obtained for all doping levels. A plot of $E_{F}-E_{v H s}$ and $T_{c}$ versus hole concentration (Fig. 6.22(b)) indicates that the Fermi level crosses the vHs near where $T_{c}$ goes to zero at around $p=0.27$. The location of the crossing has greater similarity with the single layer Bi-2201, La-214 and Tl-2201 systems than with the bilayer Bi-2212 system where the vHs crossing occurs near $p=0.22$. This can be attributed to the increased bilayer splitting in Y-123 which makes the area of the density of states probed by the Fermi window appear very much like a single-layer density of states.

In their paper[144] Williams et al. model the growing Curie-like temper- 


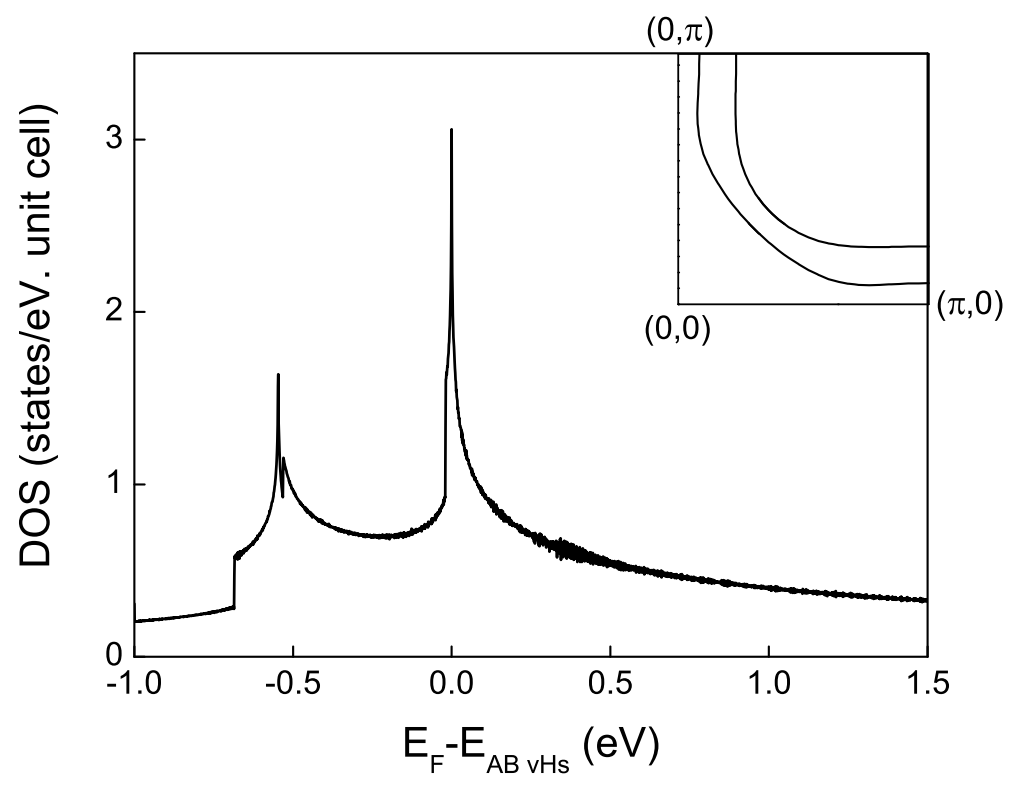

Figure 6.21: The density of states of Y-123 calculated from the energy-momentum dispersion listed in table 6.2. The inset shows the Fermi surface for $p=0.173$. In contrast to $\mathrm{Bi}-2212$, the bilayer splitting in this material is not restricted to the antinodal regions. 

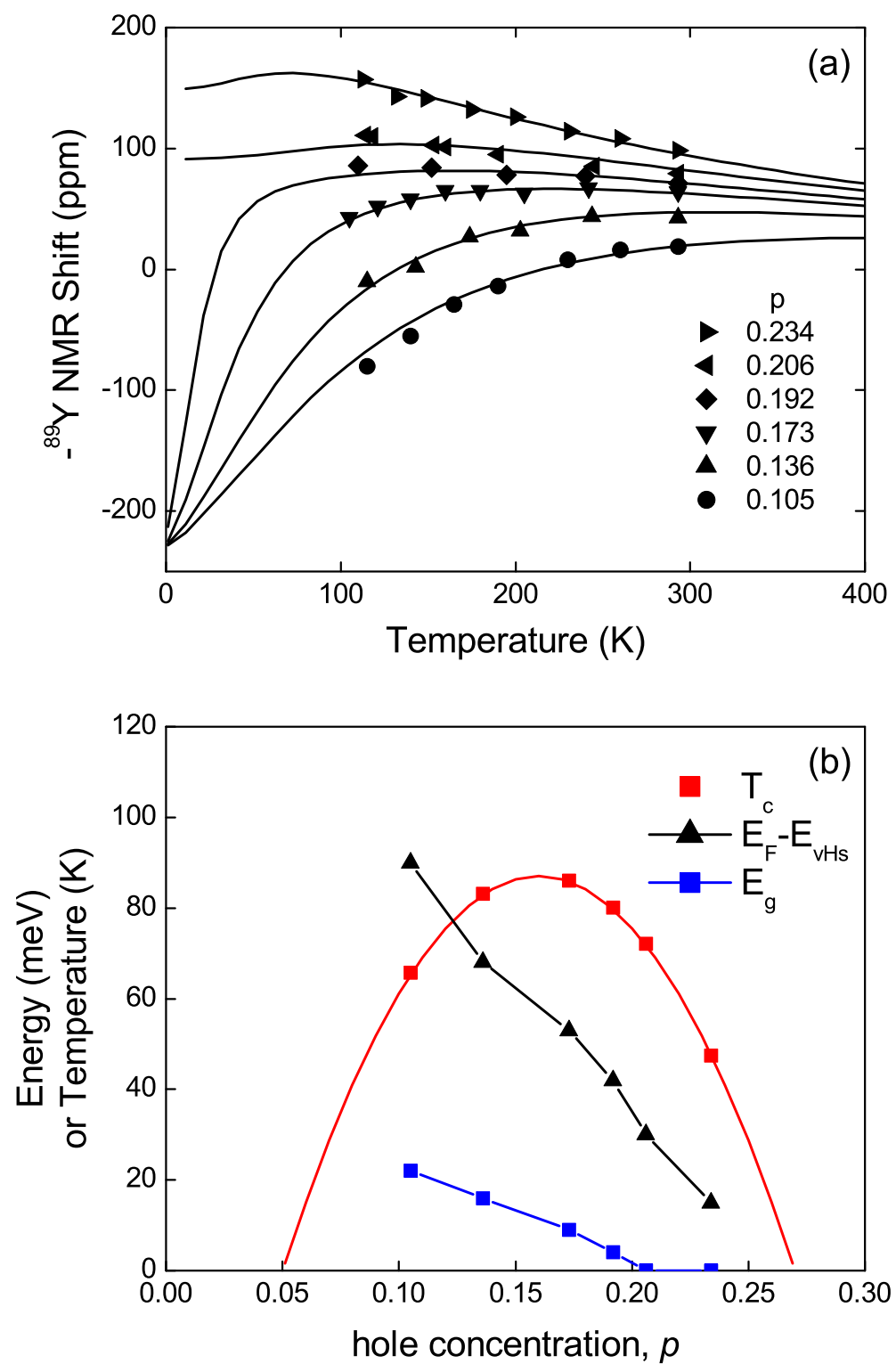

Figure 6.22: (a) Fits to the $\mathrm{Y}_{0.8} \mathrm{Ca}_{0.2} \mathrm{Ba}_{2} \mathrm{Cu}_{3} \mathrm{O}_{7-\delta}{ }^{89} \mathrm{Y}$ NMR Knight shift data of Williams et al.[144] calculated from a rigid ARPES energy-momentum dispersion and the model pseudogap of section 6.1. (b) $T_{c}$ and the values of $E_{F}-E_{v H s}$ and $E_{g}$ obtained from the fits in (a) versus hole concentration. 
ature dependence of the overdoped data with a peak in the density of states which grows with overdoping. This proposal was previously advanced by Loram et al. $[129]$ to explain the maxima in the $S\left(\mathrm{~T}_{0}\right)$ (entropy), and $\chi T\left(\mathrm{~T}_{0}\right)$ data of $\mathrm{La}_{2-x} \mathrm{Sr}_{x} \mathrm{CuO}_{4}$ as a function of doping near $\mathrm{x}=0.25$.

Under the current model the growing Curie-like temperature dependence (as well as the maxima in $S\left(\mathrm{~T}_{0}\right)$ and $\chi T\left(\mathrm{~T}_{0}\right)$ ) is clearly the result of the Fermi level traversing the vHs. The same conclusion was drawn as early as 1995 by Thoma et al.[141] who modelled the susceptibility and Knight shift using a rigid step-shaped DOS with a logarithmic vHs at the location of the step. They made the key point that conventional weak-coupling formulas for $T_{c}$ within the BCS theory are clearly not compatible with such trends in the density of states. This will be explored further in section 6.7.

\subsection{Thermoelectric Power}

The results thus far clearly reveal that the systematic trends in the thermodynamic, electrodynamic and magnetic properties reveal a universal dopingdependent behaviour that derives from the approach to and crossing of the vHs. These each involve integrals of the density of states. We turn now to another property that was early shown[146] to exhibit universal behaviour and that is the thermoelectric power. This now introduces an additional feature to the DOS, the $\mathbf{k}$-dependent scattering rate. In the following we ignore the phonon drag contribution, primarily because the computed diffusion thermopower alone adequately describes the $p$ - and $T$-dependent thermopower. 
The diffusion thermopower is given by[147]

$$
S(T)=\frac{1}{|e| T} \frac{\int_{-\infty}^{\infty} \sigma(E)(E-\mu)\left(\frac{\partial f(E)}{\partial E}\right) d E}{\int_{-\infty}^{\infty} \sigma(E)\left(-\frac{\partial f(E)}{\partial E}\right) d E}
$$

$\sigma(E)$ is the spectral conductivity which under the Boltzmann formalism is given by

$$
\sigma(E)=\frac{e^{2}}{V} \sum_{\mathbf{k}} v_{\alpha}(E, \mathbf{k}) \ell_{\alpha}(E, \mathbf{k}) \delta[\epsilon(\mathbf{k})-E]
$$

$\ell_{\alpha}$ is the mean free path given by

$$
\ell_{\alpha}=v_{\alpha}(E, \mathbf{k}) \cdot \tau(E, \mathbf{k}, T)
$$

where $v_{\alpha}=\frac{\partial \epsilon(\mathbf{k})}{\partial k_{\alpha}}$ is the group velocity of the conduction electrons, $\tau(E, \mathbf{k}, T)$ is the relaxation time and $\alpha=x$ or $y$.

In order to calculate the thermopower it is necessary to make an assumption about the form of the mean free path, $\ell_{\alpha}$. A popular starting point is to assume that it is constant[148, 57]. Under such an assumption the mean free path can be brought outside the summation in Eqn. 6.15 and is then cancelled out of the thermopower equation (Eqn. 6.14). In a recent paper Kondo et al. [57] calculated the thermopower of Bi-2201 from an ARPESderived energy-momentum dispersion. Their data is plotted in Fig. 6.23(a) along with the calculated thermopower curves which have been replicated by the present author. The calculations assume a constant mean free path and a temperature independent chemical potential $\mu$. As a first step we have corrected $\mu$ so that the carrier concentration remains constant (Eqn. 6.3) resulting in the curves shown in Fig. 6.23(b). The correction has an effect 


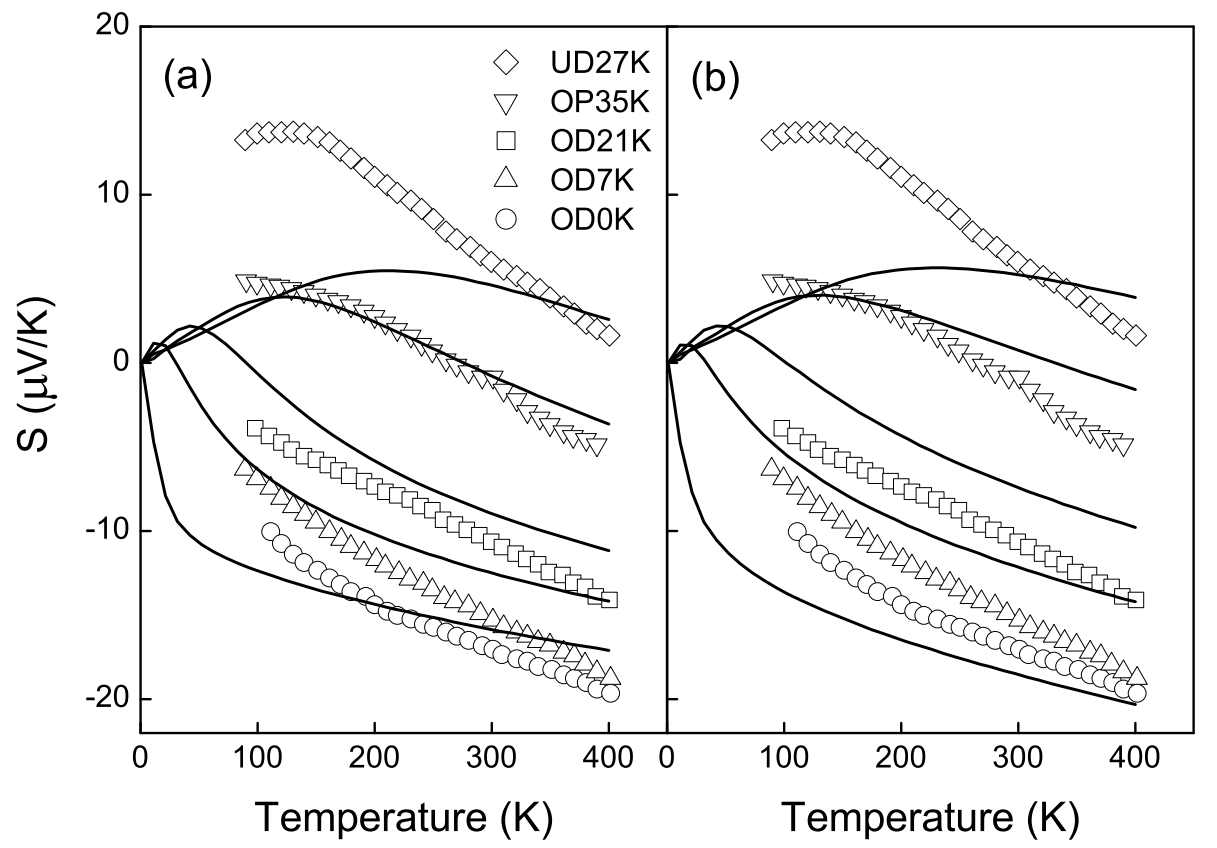

Figure 6.23: Measured and calculated thermopower of Bi-2201[57]. The curves are calculated assuming a constant mean free path. In (a) the chemical potential is constant while in (b) the chemical potential is corrected for temperature. The values of $E_{F}-E_{v H s}$ from underdoped to overdoped are $70,42,16,6$ and $-4 \mathrm{meV}$. 
on the magnitude but not the general behaviour of the thermopower. The different doping states are obtained through the adjustment of a single experimentally determined parameter, namely $E_{F}-E_{v H s}$.

Thermoelectric power is a measure of the asymmetry in the conductivity about $\mu$. The evolution of $S(T)$ with doping can be easily understood by examining the energy dependent conductivity shown in Fig. 6.24. Under the constant mean free path assumption the conductivity is proportional to $\sum v_{x} \delta[\epsilon(\mathbf{k})-E]$, which is just a velocity-weighted density of states. As in the density of states the conductivity is dominated by a peak at $E_{v H s}$. The singularity in the conductivity is not as prominent as that in the density of states due to the smaller velocities near the saddle points at $( \pm \pi, 0)$ and $(0, \pm \pi)$. At low temperatures and when the vHs is below $\mu$, the spectral weight encompassed by the spectral window $(\epsilon-\mu)[\partial f(\epsilon) / \partial \epsilon]$ below $\mu$ is greater than that encompassed above $\mu$, resulting in a positive peak in $S(T)$. As the Fermi level retreats from the vHs with underdoping this imbalance grows, thereby increasing the size and temperature extent of the peak. The peak disappears in the overdoped regime when the Fermi level crosses the vHs and so this provides a signature of the vHs crossing.

Returning to the fits, there is good qualitative and quantitative agreement with overdoped and optimally doped data. However, the calculations are unable to reproduce the rapid increase in the magnitude of $S(T)$ seen in underdoped samples. Thermopower values in excess of $300 \mu \mathrm{V} / \mathrm{K}$ have been observed in underdoped YBCO[146]. Here we explore the effects of including (i) a normal-state pseudogap and (ii) an energy and temperature dependent relaxation time. 


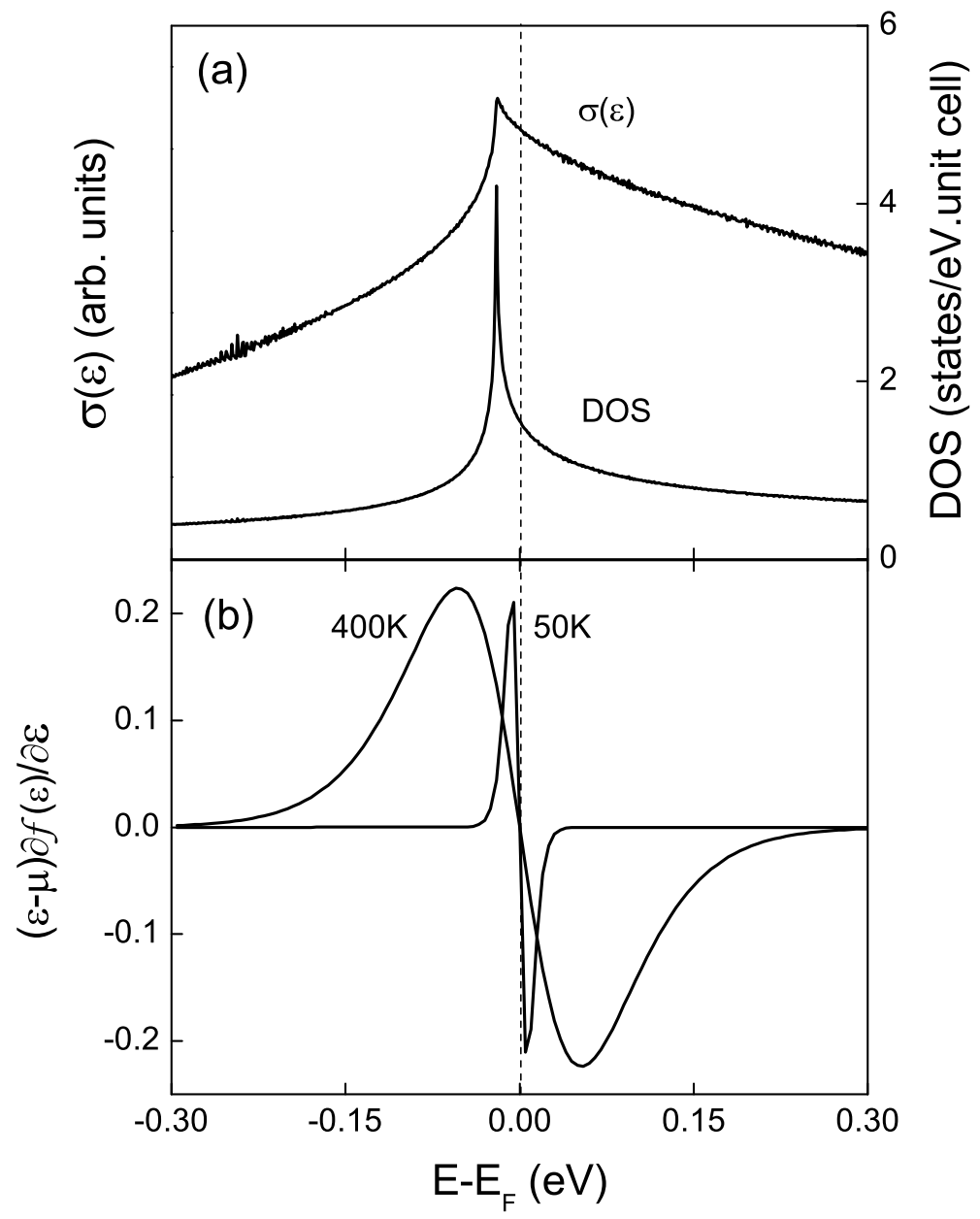

Figure 6.24: (a) The density of states of Bi-2201 calculated from the ARPESderived energy-momentum dispersion and the conductivity calculated from Eqn. 6.15 under the constant mean free path assumption. (b) The spectral window $(\epsilon-\mu)[\partial f(\epsilon) / \partial \epsilon]$ at $50 \mathrm{~K}$ and $400 \mathrm{~K}$. 


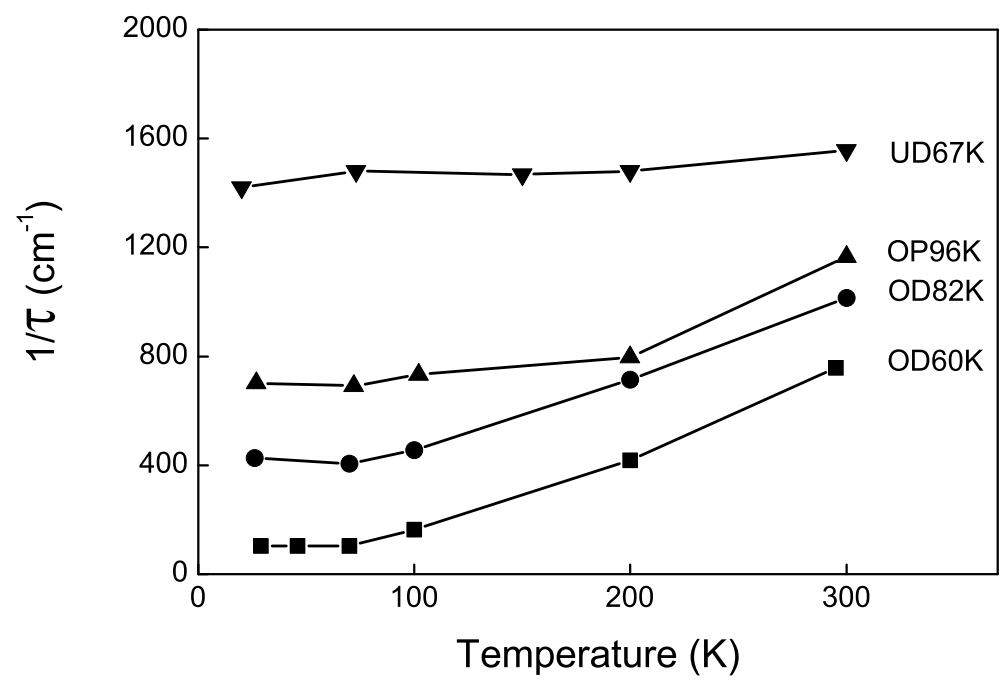

Figure 6.25: The extrapolated zero frequency scattering rate from the IR reflectivity data of Hwang et al.[149] versus temperature.

We employ the six parameter tight binding Bi-2201 dispersion reported by Kondo et al.[57] and a model for the pseudogap given by Eqns. 6.4 and 6.5. The pseudogap, as described by the model, is symmetric about $\mu$ and including the pseudogap on its own does not provide the enhanced asymmetry required to boost the magnitude of $S(T)$. This brings us to the scattering rate.

The scattering rate as determined from infrared reflectivity[149], and also FWHM analysis of ARPES spectral peaks[150], is found to be linear in energy. This together with a linear dependence on temperature, as inferred from linear resistivity, is a signature of the marginal Fermi liquid phenomenology proposed by Varma et al.[19]. The extrapolated normal-state zero frequency scattering rate from the IR reflectivity data of Hwang et al.[149] is plotted as a function of temperature in Fig. 6.25. It is observed to be temperature 
Table 6.3: Values in meV of the parameters used in the Bi-2201 thermopower fits of Fig. 6.26.

\begin{tabular}{|c|c|c|c|}
\hline Sample & $E_{F}-E_{v H s}$ & $E_{g}$ & $a$ \\
\hline OD0K & 2 & 0 & 300 \\
OD7K & 6 & 0 & 200 \\
OD21K & 16 & 0 & 200 \\
OP35K & 40 & 0 & 170 \\
UD27K & 60 & 32 & 138 \\
\hline
\end{tabular}

independent up to a certain temperature $T^{\prime}$ before becoming linear in $T$. $T^{\prime}$ increases with decreasing doping just as has been observed for $T^{*}$ and $E_{g}$. Based on the above observations the scattering rate is modelled with the following equation

$$
\frac{\hbar}{\tau}(E, T)=\frac{\pi}{2}\left[\frac{1}{\tanh \left(\frac{E_{g}}{2 k_{B} T}\right)}-1\right]+\left|E-E_{v H s}\right|+a
$$

which becomes $\hbar / \tau=\pi k_{B} T+\left|E-E_{v H s}\right|+a$ in the limit where $E_{g}$ tends to zero. $a$ is an adjustable parameter which represents intrinsic scattering. Note that in order to reproduce the peak in the conductivity at $E_{v H s}$ the scattering rate must be proportional to $E-E_{v H s}$, not $E-E_{F}$. This has an added effect of increasing the asymmetry in the conductivity about $\mu$, since $E_{v H s}$ lies below $\mu$ in the pseudogap regime. The plausibility and possible origin of this energy dependence is explored in section 6.6.

Fits to the Bi-2201 data using the above pseudogap and scattering rate models are shown in Fig. 6.26. The chemical potential is corrected for temperature. The parameters used in the fits are listed in table 6.3. The fits are improved over those made under the constant mean free path assump- 


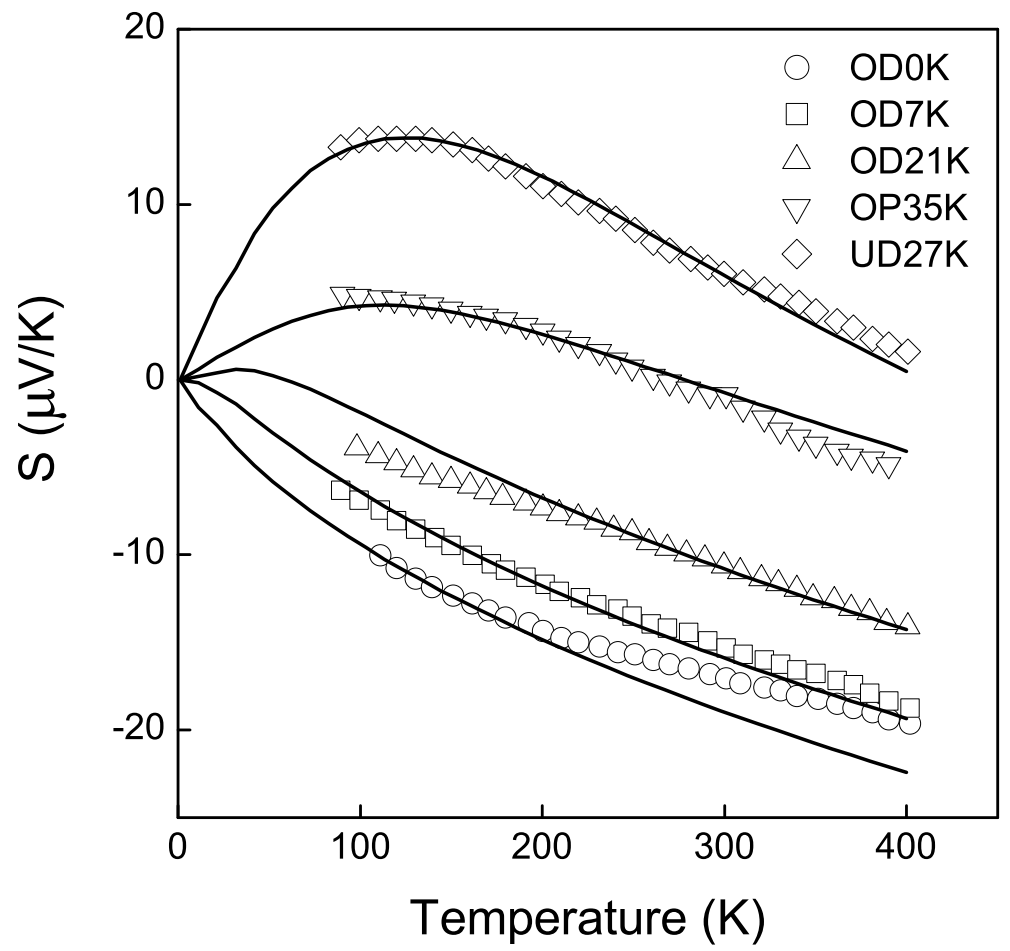

Figure 6.26: Fits to Bi-2201 data using the pseudogap model given by Eqns. 6.4 and 6.5, and a model scattering rate given by Eqn. 6.17. 
tion, not only for the underdoped data, but for all datasets. The values of $E_{F}-E_{v H s}$ are still comparable with those determined from ARPES of -4, 6, 16,42 and $70 \mathrm{meV}$.

To further test its validity the model was applied to the more abundant underdoped data of Bi-2212. Here we use the Bi-2212 dispersion of Norman et al.[32] which does not include the effect of bilayer splitting. The reason for using this simplified dispersion is that it is not clear how to apply the scattering rate model to a density of states that has a split vHs. It is expected that in the underdoped regime where the Fermi level is far from the antibonding band vHs (and even further from the bonding band vHs) that corrections due to bilayer splitting will be small. Fits to the underdoped Bi-2212 data of Munakata et al.[151] and Mandrus et al.[152]. are shown in Fig. 6.27(a). The chemical potential is corrected for temperature.

The fit parameters are plotted in Fig. 6.27(b) as a function of doping which has been estimated from the $T_{c}$ 's using the empirical relation[18] $p=0.16 \pm 0.11 \sqrt{\left(1-T_{c} / T_{c, \max }\right)}$. The model produces a good fit to the thermopower data but more importantly the parameters follow similar trends to those obtained from the entropy fits in section 6.1 (see Fig. 6.7). The scattering rate at zero frequency $\left(E=E_{F}\right)$ is plotted as a function of temperature in Fig. 6.28. The behaviour resembles the temperature dependence observed in the optical scattering rate (Fig. 6.25) however the range over which it is temperature independent is not as large.

As the doping is increased the effect of bilayer splitting becomes important and it becomes increasingly difficult to obtain a quantitative fit using the single-layer Bi-2212 dispersion. Note that this does not change the qualitative 

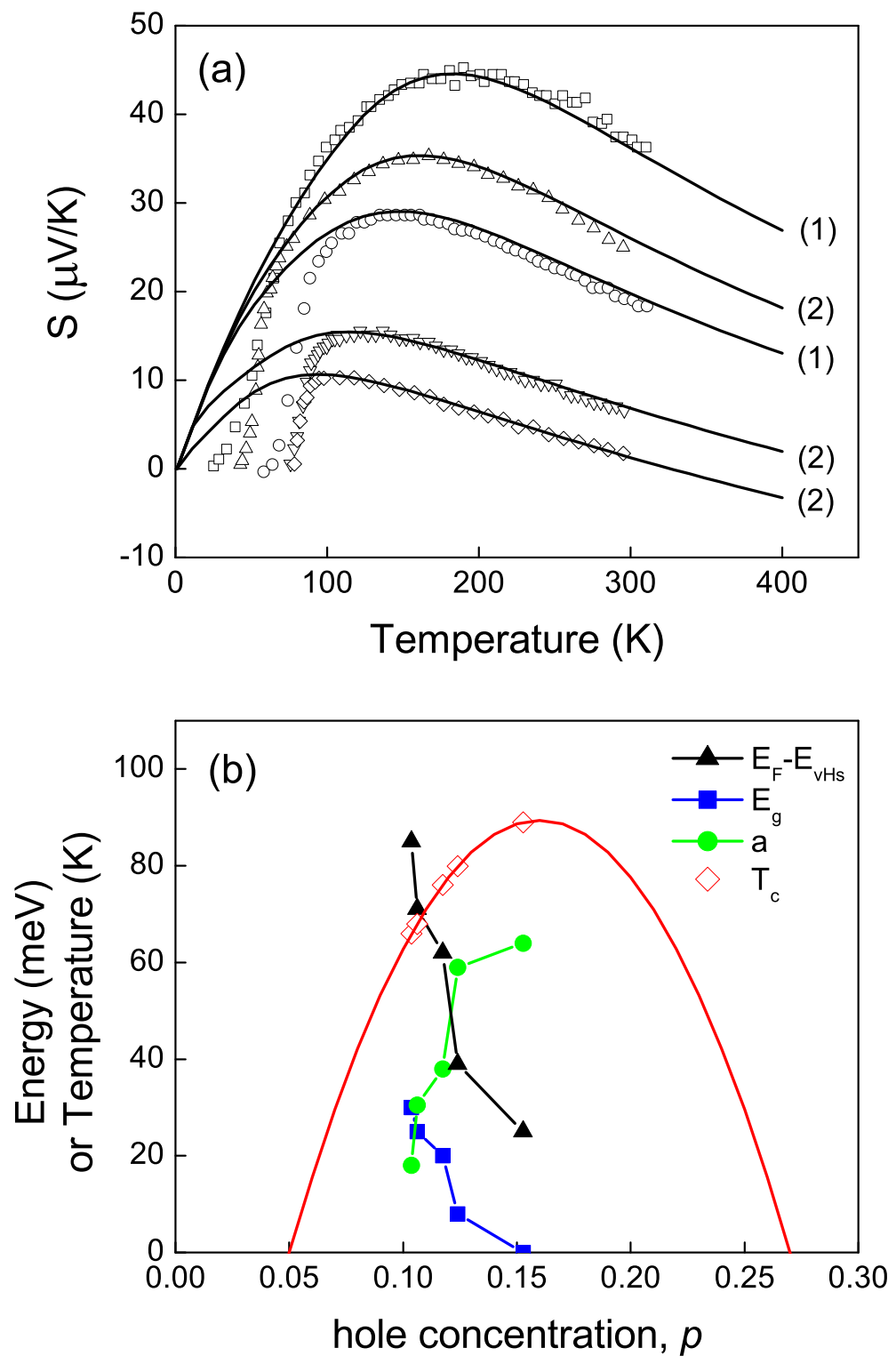

Figure 6.27: (a) Fits to underdoped Bi-2212 data of (1) Munakata et al.[151] and (2) Mandrus et al.[152]. (b) $T_{c}$ and values of the parameters used in the fits in (a) as a function of doping. 


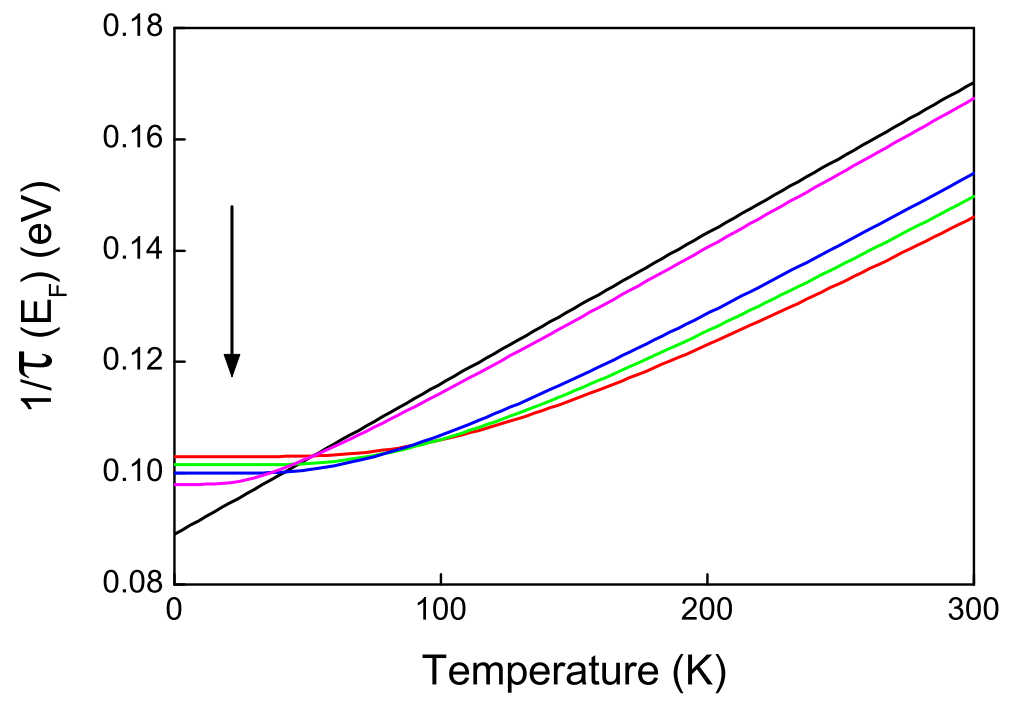

Figure 6.28: The temperature dependence of the zero frequency scattering rates used in the thermopower fits in Fig. 6.27(a). The arrow points in the direction of increasing doping.

result that the positive thermopower peak vanishes as $E_{F}$ traverses the vHs. As noted earlier the difficulty in applying the bilayer dispersion of Kaminski et al.[42] lies in deciding on a form for the scattering rate. However, as demonstrated by Kondo et al.[34], the constant mean free path assumption reproduces the behaviour of overdoped Bi-2201 thermopower data reasonably well and so we now apply this assumption to the bilayer Bi-2212 dispersion.

The conductivity is shown in Fig. 6.29. Peaks are visible at the bonding and antibonding band vHs energies. The thermopower calculated from the conductivity is shown in Fig. 6.30(b) along with comparative overdoped $\mathrm{Bi}_{1.8} \mathrm{~Pb}_{0.3} \mathrm{Sr}_{1.9} \mathrm{CaCu}_{2} \mathrm{O}_{8}$ data[153] in (a). The calculations successfully reproduce trends seen in the data, further demonstrating the reliability of the constant mean free path assumption in the overdoped regime. The calcula- 


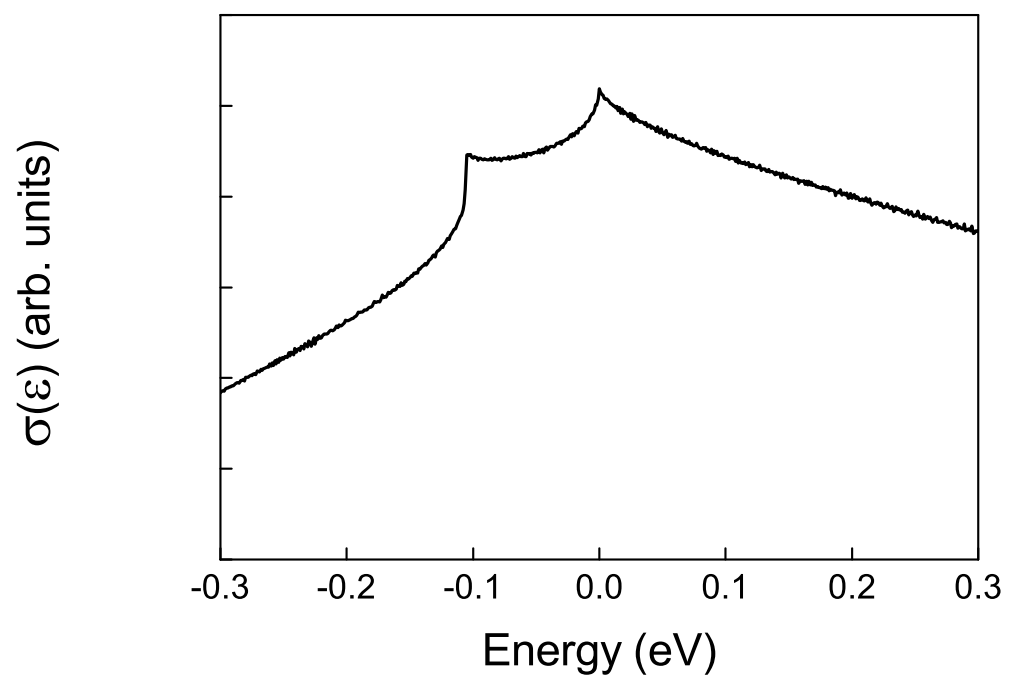

Figure 6.29: The energy dependent conductivity calculated from the bilayer Bi2212 dispersion under the constant mean free path assumption.

tions reveal that the data is consistent with the Fermi level crossing the vHs in Bi-2212 near $p=0.225\left(T_{c} \approx 60 \mathrm{~K}\right)$ as deduced from ARPES[42] and fits to the electronic entropy in section 6.1. The deviation from linearity between $100 \mathrm{~K}$ and $300 \mathrm{~K}$ is found to be due to the influence of the bonding band conductivity peak and is clearly visible in the data. The same curvature is seen in the overdoped data of Munakata et al.[151].

The fact that an exact fit is not obtained is most likely due to the constant mean free path assumption. This assumption was made in order to keep the number of assumptions to a minimum. The gradients of the calculated curves are not as steep as those of the data, but, as seen in Fig. 6.23, this situation also occurs for Bi-2201. In principle, exact fits could be made by choosing appropriate scattering rates for the bonding and antibonding bands.

This work builds on the approach taken by McIntosh \& Kaiser[148] and 


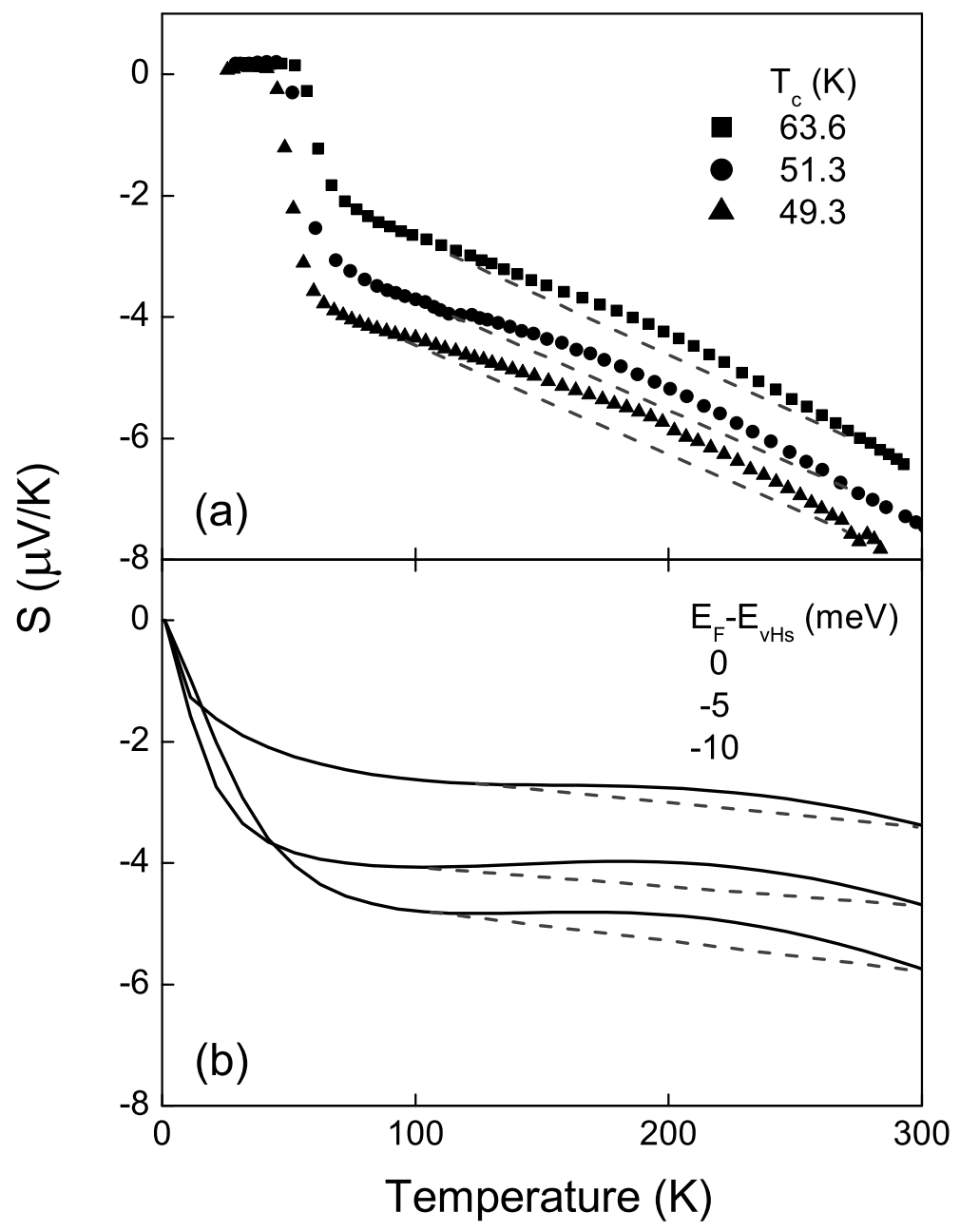

Figure 6.30: (a) Overdoped $\mathrm{Bi}_{1.8} \mathrm{~Pb}_{0.3} \mathrm{Sr}_{1.9} \mathrm{CaCu}_{2} \mathrm{O}_{8}$ thermopower data[153]. (b) The thermopower of $\mathrm{Bi}-2212$ calculated under the constant mean free path assumption from a bilayer Bi-2212 dispersion. The dotted lines are included to highlight the curvature between 100 and 300K. 
Kondo et al. [34] but differs from the phonon drag approach taken by

Trodahl[154]. In the phonon drag model the thermopower is explained as a combination of a negative linear diffusion part and a positive phonon drag contribution which initially rises linearly with temperature before saturating at high temperatures. Under that model it is deduced that "the diffusion thermopower is clearly negative for all hole concentrations and proportional to temperature for the majority of cuprates." The model developed here shows that this is not the case and highlights the importance of including a realistic band structure.

By taking into account the energy momentum dispersion and a model for the single particle scattering rate it has been shown that evolution of the thermopower in the cuprates can be explained by the diffusion component alone and implies that the phonon drag component is small.

\subsection{Scattering Rate}

In the previous section it was shown that with an appropriate scattering rate the doping and temperature dependence of the thermopower, $S(T, p)$, can be explained by the diffusion component alone. As shown by Kondo et al. [34] the doping dependence from the optimal to overdoped regimes can be approximately reproduced by assuming a constant mean free path and sliding the Fermi level towards the vHs. In the model, the van Hove singularity at energy $E_{v H s}$ gives rise to a peak in the conductivity at $E_{v H s}$ which is responsible for the behaviour of $S(T)$. While extending the model, by including a linear-in-energy scattering rate, it was found that in order to reproduce 


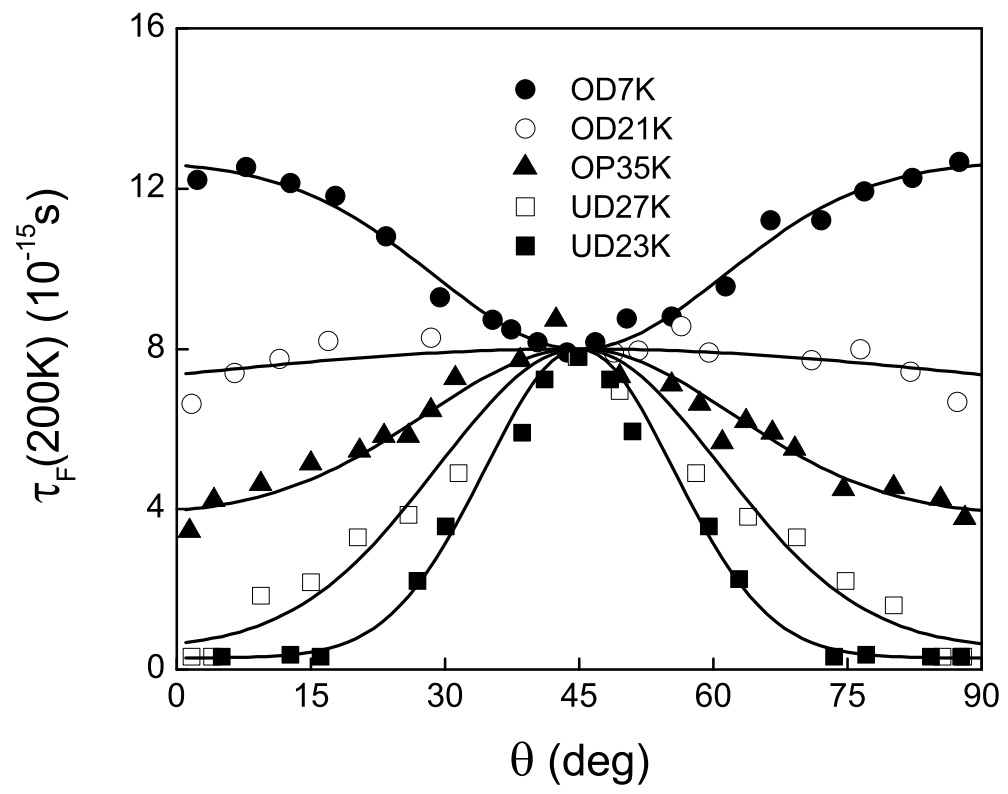

Figure 6.31: Lifetime of the Bloch states at the Fermi level $\tau_{F}(\theta)$ determined from ARPES measurements at 200K[155]. The curves are fits given by Eqn. 6.18.

the peak in the conductivity at $E_{v H s}$ the scattering rate needed to be proportional to $E-E_{v H s}$, not $E-E_{F}$. Here the momentum dependence of the scattering rate is investigated to find out if it does indeed give rise to a scattering rate that is proportional to $E-E_{v H s}$.

Figure 6.31 shows relaxation time plotted as a function of Fermi surface angle for underdoped to overdoped Bi-2201 samples as determined from ARPES measurements at 200K[155]. It is immediately apparent that the relaxation time around the Fermi surface increases as the doping increases. In the most overdoped sample with $T_{c}=7 \mathrm{~K}$ (filled circles) the relaxation time is maximal at 0 and 90 degrees. As the Fermi level lies close to the vHs at this doping this indicates that the scattering rate is small near the saddle points. 
It is tempting to associate this behaviour of the scattering rate with the evolution of the pseudogap where the scattering rate is maximal near $(\pi, 0)$ (perhaps due to scattering from spin fluctuations). A combination of the sharp reduction in $J$ with doping (see section 6.7) and spin flip scattering due to mobile electrons will progressively reduce this zone boundary scattering rate, and notably, the scattering rate is isotropic when the pseudogap closes (open circles in Fig. 6.31). In the more overdoped region, if the mean free path becomes constant then the reduction in Fermi velocity near $(\pi, 0)$ as the vHs is approached would account for the rise in $\tau_{F}\left(\theta=0,90^{\circ}\right)$ in the most overdoped sample.

To obtain an analytical expression for the relaxation time the data was fitted with the following equation

$$
\tau(\theta)=\Omega\left[\exp \left(-\frac{\left(\theta-45^{\circ}\right)^{2}}{\sigma^{2}}\right)-1\right]+8
$$

The fit parameters $\Omega$ and $\sigma$ are plotted as a function of $E_{F}-E_{v H s}$ in Fig. 6.32. Also plotted is a fit to the $\Omega$ values given by

$$
\Omega= \begin{cases}{\left[-15.936\left(1-\tanh \left[19.421\left(E_{F}-E_{v H s}\right)\right]\right)\right.} & \\ +9.4008], & {\left[0<\left(E_{F}-E_{v H s}\right)<0.08\right]} \\ -6.5352, & {\left[\left(E_{F}-E_{v H s}\right) \leq 0\right]}\end{cases}
$$

where $\left(E_{F}-E_{v H s}\right)$ is in $\mathrm{eV}$. The value of $\Omega$ at $20 \mathrm{meV}$ has been omitted from the fit and the fit is valid up to $80 \mathrm{meV}$. Beyond this point the values of $\Omega$ produced by this equation result in negative relaxation times when 


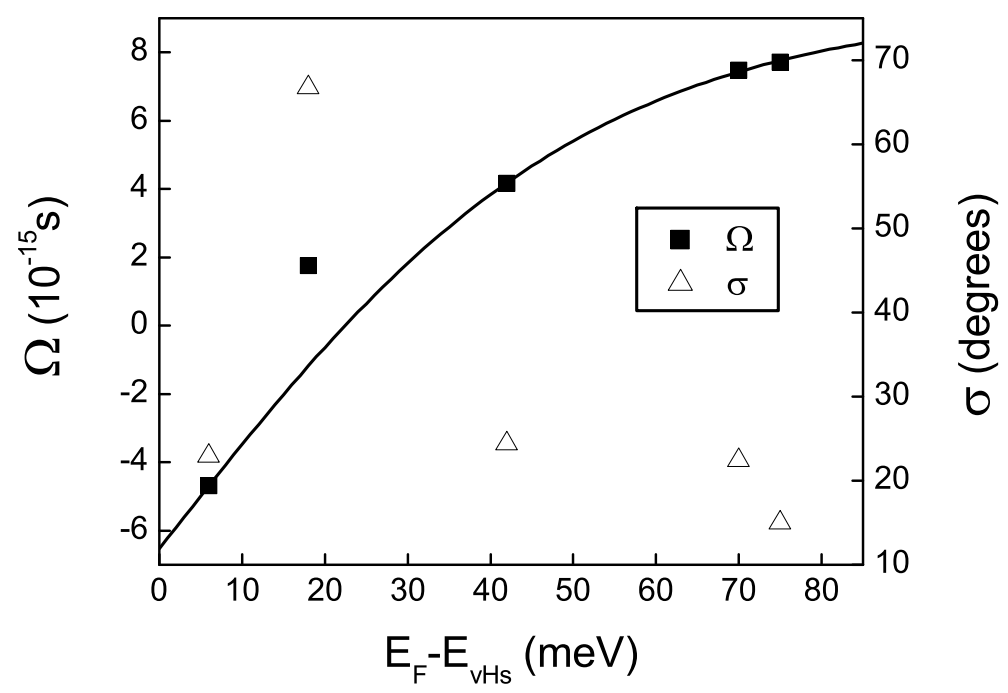

Figure 6.32: The parameters of the fits shown in Fig. 6.31 as a function of $E_{F}-$ $E_{v H s}$. The black curve is a fit to $\Omega$ given by Eqn. 6.19

substituted into Eqn. 6.18.

The conductivity can now be calculated from the relaxation time given by Eqn. 6.18 together with $\Omega$ given by Eqn. 6.19. $\sigma$ is set to $25^{\circ}$. The result is shown in Fig. 6.33. The peak at $E_{v H s}$ is reproduced, demonstrating that the apparent $E-E_{v H s}$ dependence of the scattering rate is simply an artifact of a more complex underlying momentum dependent relaxation time. However, $E_{v H s}$ is still included explicitly in this parametrization of the relaxation time and so we look for a purely momentum dependent expression that reproduces the relaxation time observed by ARPES.

The velocity field $\left(\frac{\partial \epsilon}{\partial k_{x}}, \frac{\partial \epsilon}{\partial k_{y}}\right)$ is plotted in Fig. 6.34. The velocity field suggests that the electrons prefer to travel in the diagonal direction and that transport is frustrated when travelling in the $(0,0)-(\pi, 0)$ and $(0,0)-(0, \pi)$ directions. As mentioned by Valla et al.[156], such a situation might arise 


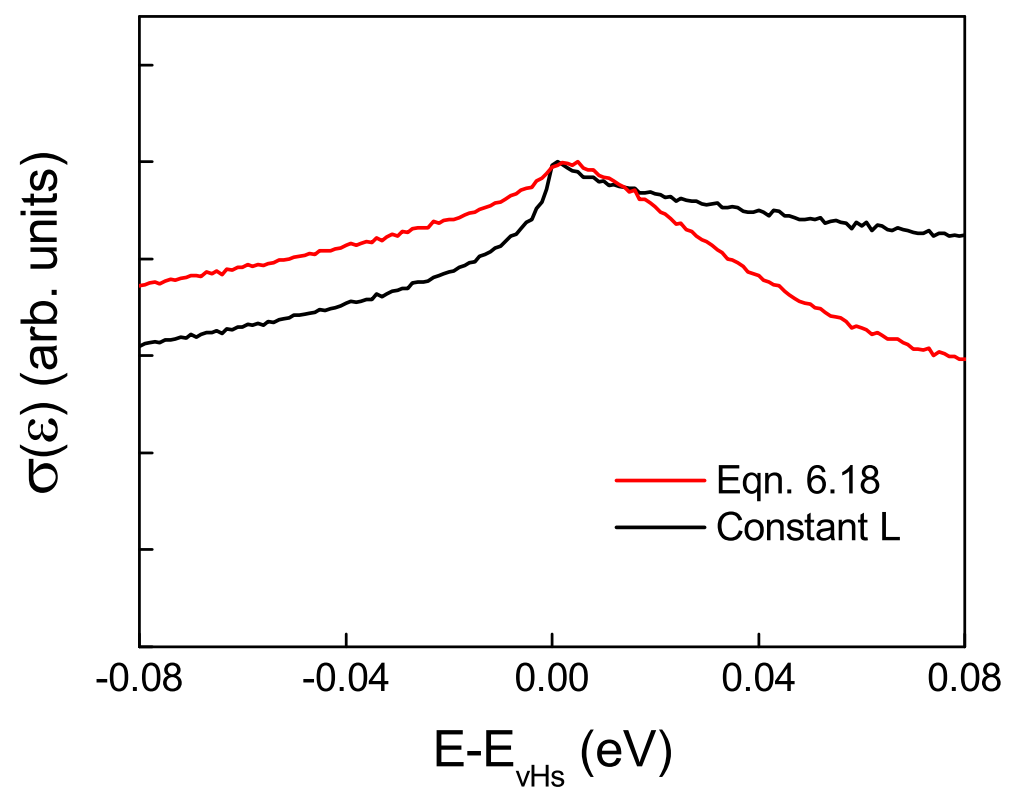

Figure 6.33: The conductivity calculated from Eqn. 6.15 with and ARPES-derived $\tau$ given by Eqn. 6.18 (red). The conductivity calculated under the constant mean free path assumption is shown in black. 


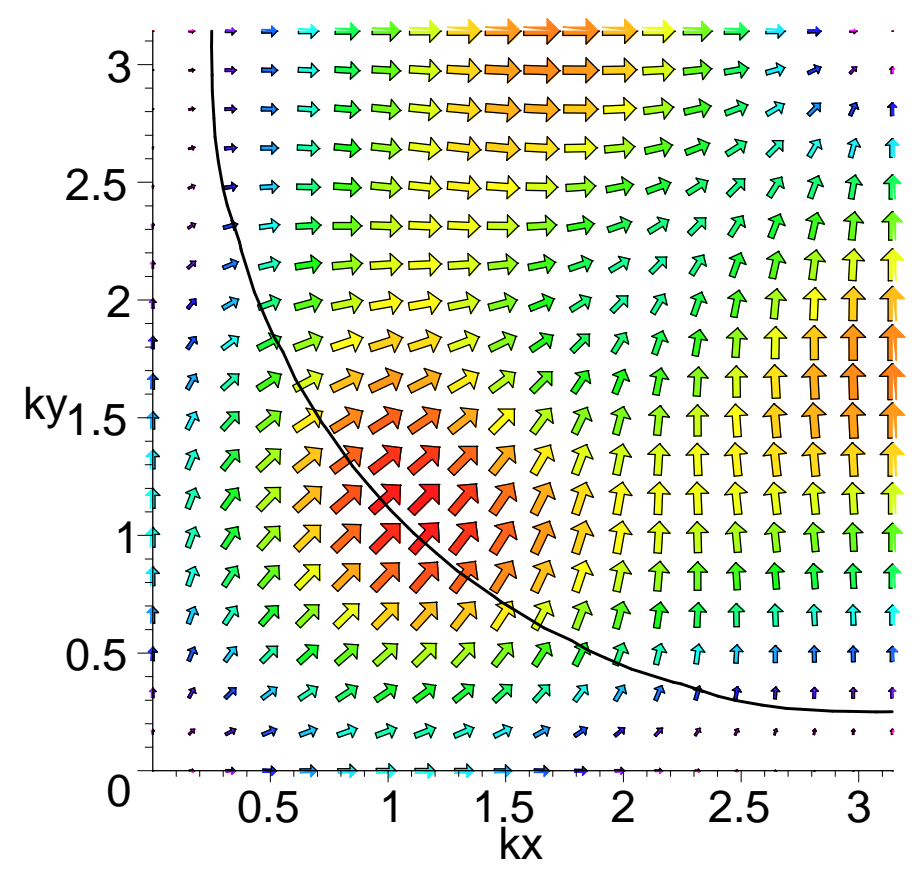

Figure 6.34: The velocity field $\left(\frac{\partial \epsilon}{\partial k_{x}}, \frac{\partial \epsilon}{\partial k_{y}}\right)$ of Bi-2201. The vectors are coloured according to their magnitude. The black curve shows the constant energy contour $\epsilon-E_{v H s}=20 \mathrm{meV}$. 


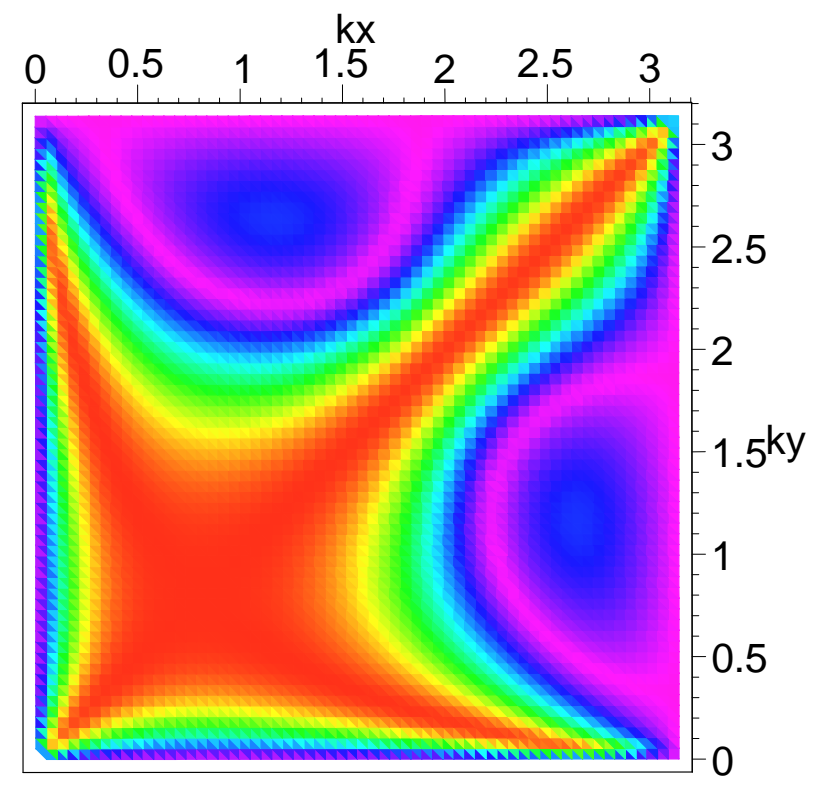

Figure 6.35: The cosine of the angular deviation of the velocity from $45^{\circ}$. Violet $=0$, red $=1$.

from the underlying antiferromagnetic structure of the cuprates. Along the diagonal direction the spins on neighbouring copper sites are ferromagnetically aligned. Whereas along the copper-oxygen bond direction transport is frustrated by the antiferromagnetic alignment of the spins on neighbouring copper sites. We therefore construct a momentum dependent relaxation time based on the velocity field as follows. The angle subtended by a given velocity vector with the $k_{x}$ axis given by $\phi=\tan ^{-1}\left(v_{y} / v_{x}\right)$. The deviation of $\phi$ from the $(0,0)-(\pi, \pi)$ diagonal direction is $45^{\circ}-\phi$. Preferential nodal transport can then be reflected in the relaxation time mathematically by setting $\tau \propto \cos \left[2\left(45^{\circ}-\phi\right)\right]=\sin (2 \phi)$ which is plotted as a function of $\left(k_{x}, k_{y}\right)$ in Fig. 6.35. Figure 6.36 shows $\sin (2 \phi)$ plotted as a function of Fermi surface angle for different values of $E_{F}-E_{v H s}$. The qualitative behaviour has a rough 


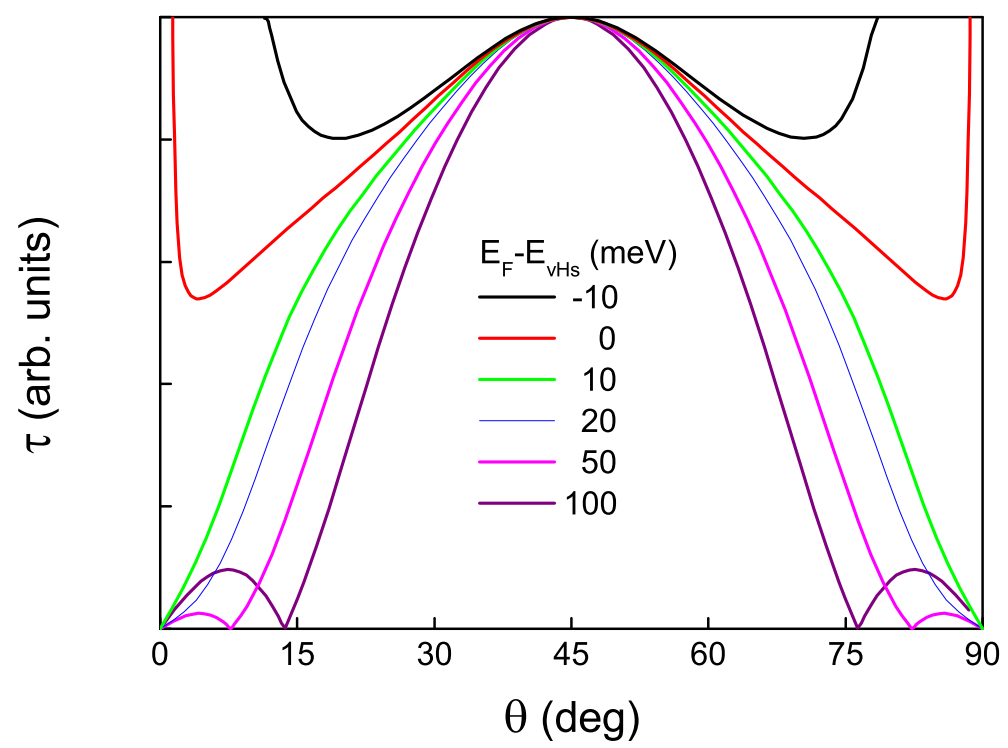

Figure 6.36: The cosine of the angular deviation of the velocity from $45^{\circ}$ plotted as a function of Fermi surface angle for different dopings.

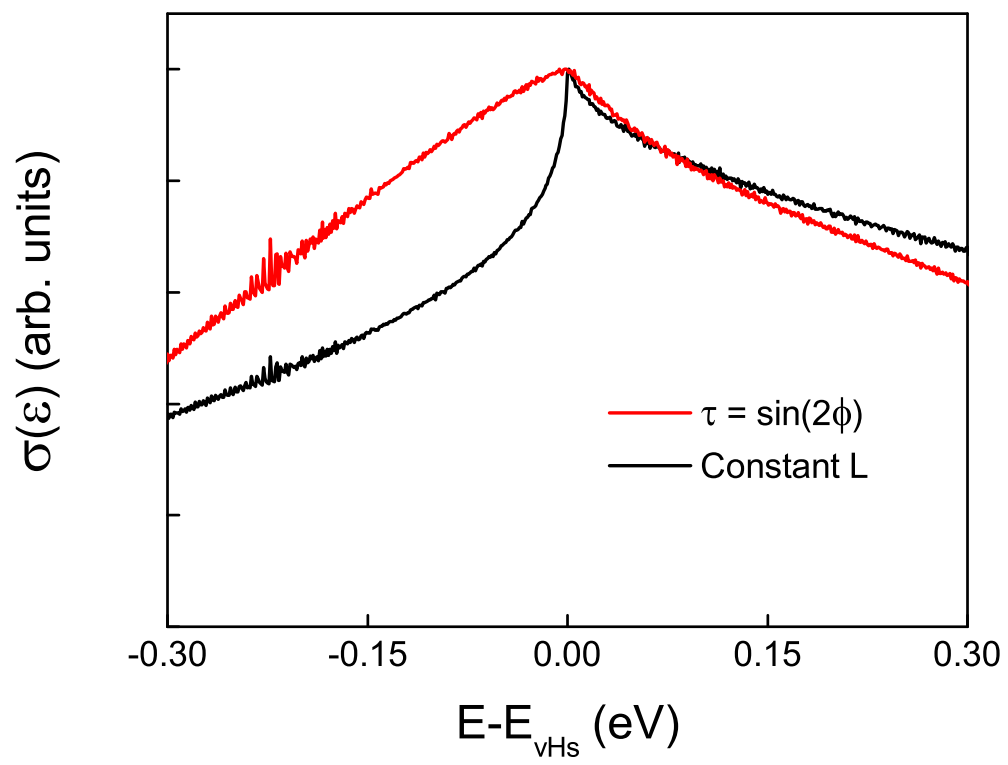

Figure 6.37: The conductivity calculated from Eqn. 6.15 with $\tau$ given by $\cos \left[2\left(\phi-45^{\circ}\right)\right]$ (red). The conductivity calculated under the constant mean free path assumption is shown in black. 
resemblance to the experimental data shown in Fig. 6.31. The conductivity calculated using this form for the relaxation time is shown in Fig. 6.37. The peak in the conductivity at $E_{v H s}$ is reproduced further demonstrating that the unusual $E-E_{v H s}$ dependence of the scattering rate can be accounted for by considering the momentum dependence of the relaxation time.

\subsection{Phase Diagram}

Fits to the normal state electronic entropy (section 6.1), spin susceptibility (section 6.4) and thermoelectric power (section 6.5) confirmed that the Fermi level crosses a van Hove singularity in the deeply overdoped regime. Fits to the superconducting state electronic entropy revealed that the pairing potential cut-off energy, $\omega_{c}$, is linearly related to the distance of the Fermi level from the van Hove singularity. Now we turn to the magnitude of $T_{c}$ itself and the generic superconducting phase diagram. Within a BCS picture the transition temperature is given by[3]

$$
k_{B} T_{c}=1.14 \hbar \omega \exp \left(-\frac{1}{N\left(E_{F}\right) V}\right)
$$

so that if the vHs crossing occurs within the superconducting domain the exponential dependence upon the density of states should result in a local peak in $T_{c}$ precisely at the vHs. In this section the superconductivity phase diagram is calculated and it is shown that if the Fermi energy crosses the van Hove singularity in the overdoped regime then the pairing potential or the pairing energy cut-off must be a strong decreasing function of hole con- 
centration.

The doping dependence of $T_{c}$ is calculated for Bi-2212 as follows. We employ the ARPES derived Bi-2212 bilayer dispersion, $\epsilon(\mathbf{k})$, listed in table 6.1. The position of the Fermi level as a function of doping is obtained from the fits to the entropy in section 6.1. The pairing potential is assumed to be of the form $V_{\mathbf{k k}^{\prime}}=V g(\mathbf{k}) g\left(\mathbf{k}^{\prime}\right)$ where $g(\mathbf{k})=\cos k_{x}-\cos k_{y} . T_{c}$ is calculated solving the weak-coupling BCS gap equation for $T$ in the case where $\Delta_{0}=0$ given by

$$
1=\frac{V}{2} \sum_{\mathbf{k}} \frac{|g(\mathbf{k})|^{2}}{\epsilon(\mathbf{k})} \tanh \left(\frac{\epsilon(\mathbf{k})}{2 k_{B} T}\right)
$$

The summation is performed over states with energy $|\epsilon(\mathbf{k})|<\omega_{c}$. The energy cut-off $\omega_{c}$ is assumed to be constant and is set to a value of $70 \mathrm{meV}$. This is the energy at which a renormalization or 'kink' occurs in the dispersion (see section 2.7).

The weak-coupling assumption is suitable for our purposes and the qualitative results would not alter with the introduction of strong coupling. However, there is in fact important thermodynamic evidence for a weak coupling scenario[157].

Two cases are considered: (i) the pairing amplitude, $V$, is constant, chosen such that $T_{c, \max }$ takes the observed value; and (ii) for each $p$-value $V$ is selected such that $T_{c}(p)$ follows the experimentally-observed, approximately parabolic, phase curve. Fig. 6.38(a) shows $T_{c}(p)$ plotted as a function of hole concentration for the two cases.

Turning first to the $T_{c}(p)$ curve for a constant $V$ (up-triangles), it is evident that if the pairing interaction and cut-off energy is fixed then the phase 

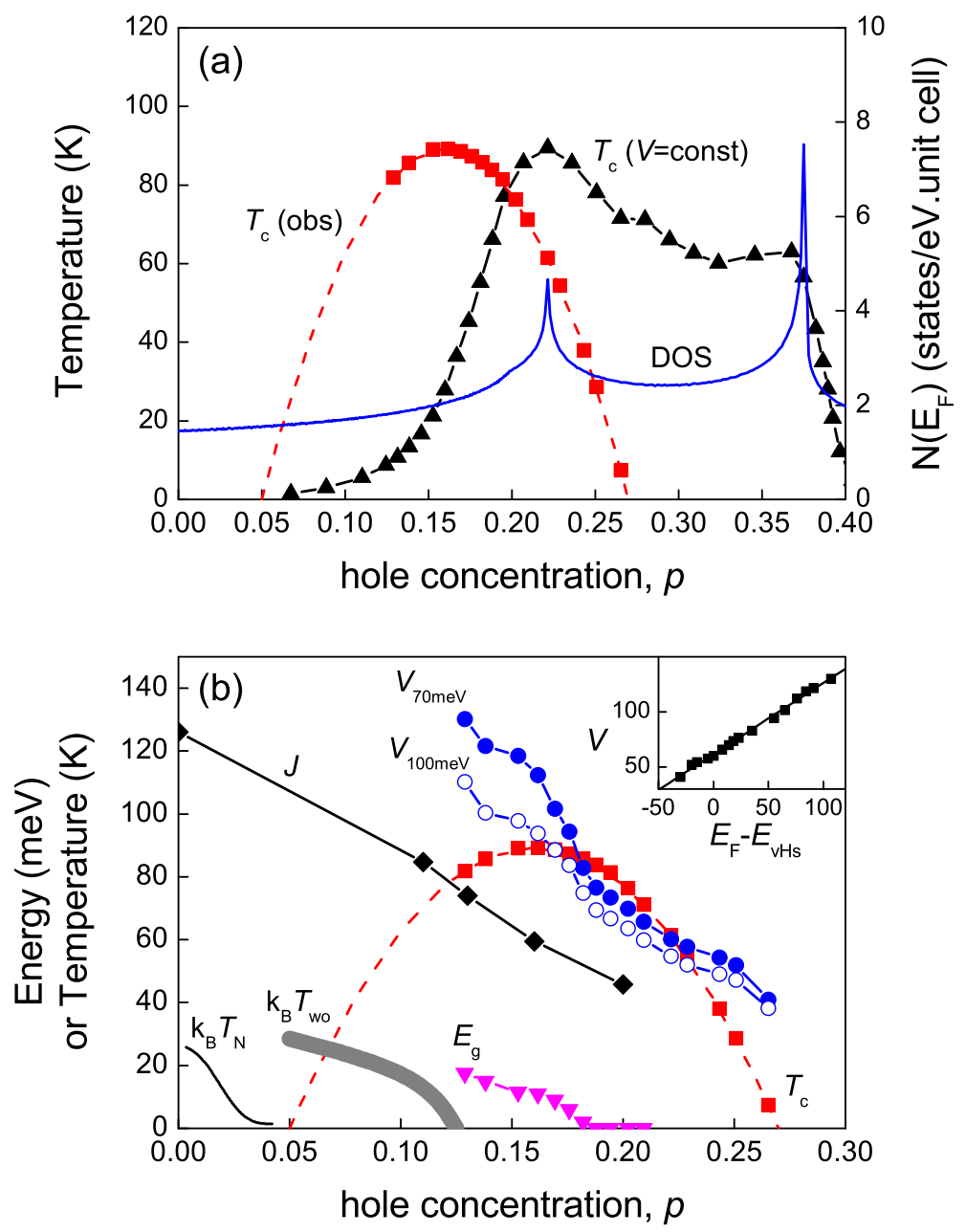

Figure 6.38: (a) The doping dependence of the DOS $N\left(E_{F}\right)$ and of $T_{c}$, as observed (squares) and as calculated (triangles) assuming a constant pairing interaction. (b) the doping dependence of the pairing amplitude, $V$, and of the exchange energy $J$, the pseudogap energy $E_{g}$, the NMR wipe-out line $k_{B} T_{w o}$ and Néel line $k_{B} T_{N}$. 
curve (a) is more narrow than that which is observed, (b) maximises at the location of the vHs in the heavily overdoped region (not at optimal doping) and (c) exhibits a second peak at the bonding-band vHs. All three difficulties are averted, and the peak broadened and shifted back to the observed optimal doping, only if either the pairing interaction or the energy cut-off decreases rapidly with increasing doping. This is an important and robust result independent of the particular details that follow. This is illustrated by the second case explored, as follows.

The second $T_{c}(p)$ phase curve (red squares) follows the empirical, approximately parabolic, phase curve[18]

$$
T_{c}=T_{c, \max }\left[1-82.6(p-0.16)^{2}\right]
$$

Using the gap equation, the values of $V$ that reproduce these $T_{c}$ values were calculated and are shown by the circles plotted as a function of $p$ in Fig. 6.38(b). They descend rapidly towards zero with increasing doping. If, alternatively, $V$ is held constant and the energy cut-off, $\omega_{c}$, is varied, essentially the same result is obtained - a rapidly descending value that vanishes near $p \approx 0.3$ (see Fig. 6.7).

The pairing potential, $V$, is clearly large and grows with underdoping towards the magnitude of $J$, the exchange interaction. For comparison, the magnitude of $J$ determined from two-magnon Raman scattering[158] is plotted in Fig. 6.38(b), (where $J$ is taken, as usual, as $1 / 3$ the frequency of two-magnon scattering peak). The magnitude and doping dependence of $V$ is very similar to that of $J$, suggesting a close relationship between these. 
Also plotted (open circles) is $V$ when $\omega_{c}=100 \mathrm{meV}$. A similar rapid fall is found showing that the choice of $\omega_{c}$ is not too critical.

This rapid fall in energy scales with doping is also reflected in several other energy scales also shown in Fig. 6.38(b). These are the pseudogap energy scale, $E_{g}$ and the line $T_{w o} / k_{B}$ where $T_{w o}$ is the temperature where NMR intensity wipe-out effects are observed, indicating the onset of inhomogeneous spin and charge distribution[159, 160]. It is also in this region that the $4 \times 4$ checkerboard structure is observed in scanning tunneling spectroscopy[161]. The values of $T_{w o}$ are for (Y,Ca) $\mathrm{Ba}_{2} \mathrm{Cu}_{3} \mathrm{O}_{7-\delta}[160]$. These lines all expand out from the antiferromagnetic phase curve, $T_{N}(p)[162]$, like ripples of remnant magnetic effects suggesting a common magnetic origin for all these energy scales.

Here the doping is estimated from the parabolic phase curve which is known to be approximate only. In fact $V$ is very linear in $E_{F}-E_{v H s}$, as shown in the inset to Fig. 6.38(b). This suggests that the overall phase curve $T_{c}(p)$ is indeed governed by the proximate $\mathrm{vHs}$ combined with a rapidly declining bosonic energy scale. The value of $\omega_{c}$ or $V$ need not vanish at $p \approx 0.27$. Eventually the superconducting energy gap will fall below the pairbreaking scattering rate and $T_{c}$ will be reduced to zero[81] even if $\omega_{c}$ or $V$ are not quite zero.

The doping dependence of $T_{c}$ and the pairing potential amplitude $V$ can be understood by looking at the energy-resolved contributions to the BCS 
gap equation given by

$$
\zeta(E)=\sum_{\mathbf{k}} \frac{|g(\mathbf{k})|^{2}}{E(\mathbf{k})} \tanh \left(\frac{E(\mathbf{k})}{2 k_{B} T}\right) \delta[\epsilon(\mathbf{k})-E]
$$

The BCS gap equation is then given by

$$
1=\frac{V}{2} \int_{-\omega_{c}}^{\omega_{c}} \zeta(E) d E
$$

In the underdoped regime, for a given $\Delta$, as $E_{F}$ recedes from the vHs the area under $\zeta(E)$ decreases and so $V$ has to increase (see Fig. 6.39(a)). Whereas in the overdoped regime, for a given value of $E_{F}-E_{v H s}$, as $\Delta$ reduces the area under $\zeta(E)$ increases and so $V$ has to decrease (see Fig. 6.39(b)). It is the interplay between the recession of $E_{F}$ from the $v H s$ and the increase in $V$ with decreasing doping that results in the parabolic dependence of $T_{c}$.

The decrease in the deduced bosonic energy scale or interaction strength will be reflected in a similar decline in the magnitude of the electron-boson coupling parameter $\lambda=N\left(E_{F}\right) V$ (or its $\mathbf{k}$-dependent analogue). It is notable therefore that ARPES measurements of the ratio of the renormalized velocity at $E_{F}$ to the unrenormalized value well below $E_{F}$ shows a similar rapid decline with doping[93]. This ratio is a measure of (though overestimates) the coupling strength. It seems clear therefore from our calculations and ARPES results that the electron-boson coupling strength indeed falls rapidly with doping across the phase diagram. This combination of a rapid fall in $V$ combined with the rise in density of states as the vHs is approached thus generally accounts for the parabolic $T_{c}(p)$ phase diagram. This would suggest 


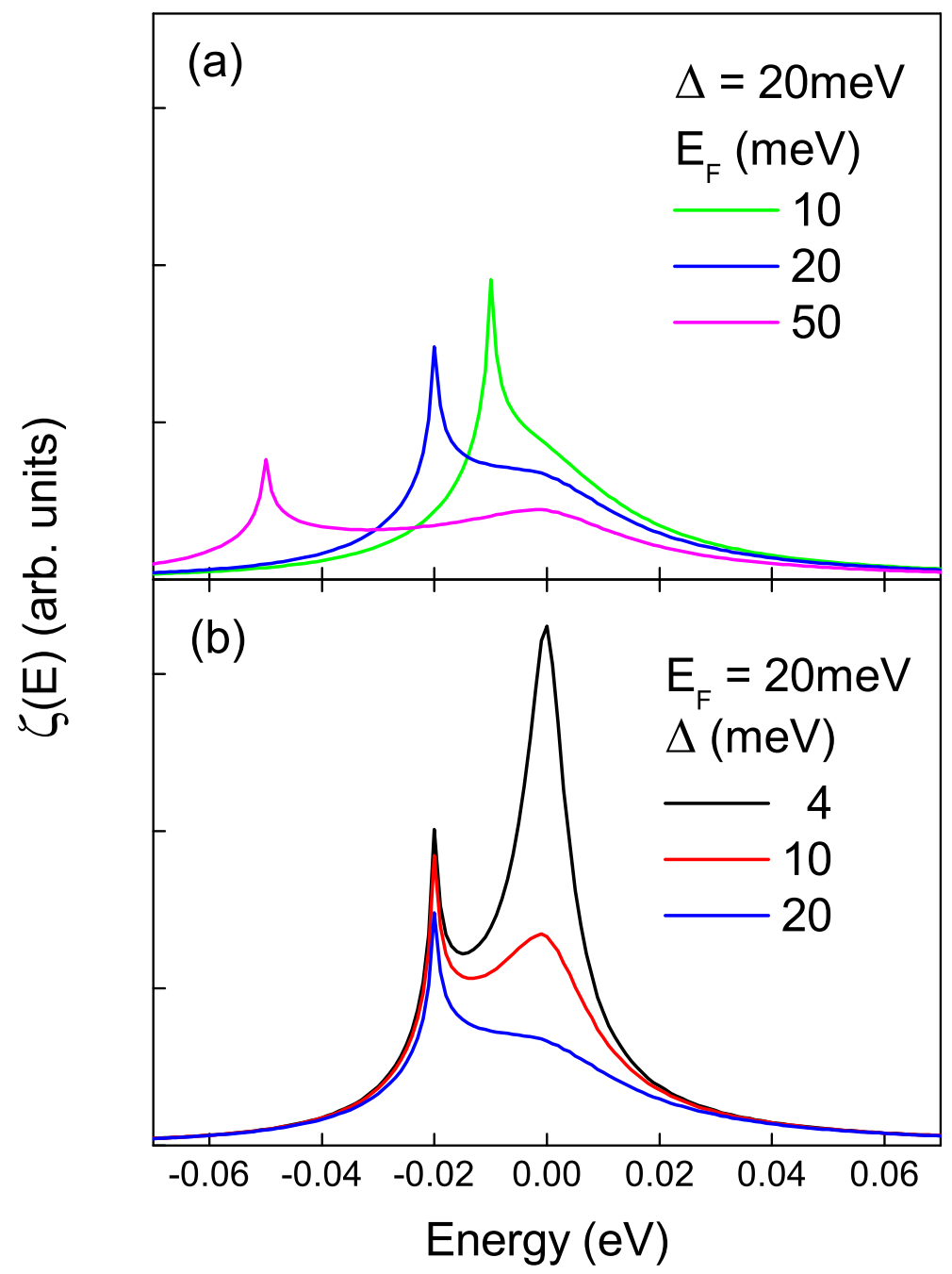

Figure 6.39: (a) At fixed $\Delta$, as $E_{F}$ recedes from the vHs the area under $\zeta(E)$ decreases and so $V$ has to increase. (b) At fixed $E_{F}$, as $\Delta$ reduces the area under $\zeta(E)$ increases and so $V$ has to decrease. 
that a prescription for increasing $T_{c, \max }$ would be to modify the structure so as to shift the vHs to lower doping so that the higher density of states locks into a higher pairing interaction.

By calculating the band structure of a large number of the single-layer cuprates Pavarini et al.[31] have found a positive correlation between the ratio of the next-nearest-neighbour to nearest-neighbour hopping parameters, $t^{\prime} / t$, and the observed $T_{c, \max }$. The origin of this correlation was investigated using the Bi-2201 ARPES data of Kondo et al[57]. In their paper Kondo et al. list the tight binding parameters for the Bi-2201 energy-momentum dispersion as well as $E_{F}-E_{v H s}$ and $T_{c}$ values for several doping levels. From this data $V$ was calculated assuming a rigid band and a fixed pairing potential energy cut-off $\omega_{c}=70 \mathrm{meV}$. Having thus determined $V$ as a function of $E_{F}-E_{v H s}$, $T_{c}$ was recalculated for two different values of $t^{\prime}$ (one slightly larger and one slightly smaller). The hole concentration was calculated from the area enclosed by the Fermi surface, $A_{p F S}$, using the relation[57]

$$
p=2\left(\frac{A_{p F S}}{A_{B Z}}\right)-1
$$

where $A_{B Z}$ is the area of the first Brillouin zone.

The resulting $T_{c}$ curves are plotted in Fig. 6.40(a). The absolute magnitude of $p$ increases with $t^{\prime} / t$ and so for comparison the curves are plotted as a function of $p / p_{\text {opt }}$. $T_{c, \text { max }}$ is observed to increase with $t^{\prime} / t$ in agreement with the results of Pavarini et al. A comparison of the densities of states (Fig. 6.40(b)) reveals that the increase in $T_{c, \max }$ is due to a net increase in the density of states with $t^{\prime} / t$. 

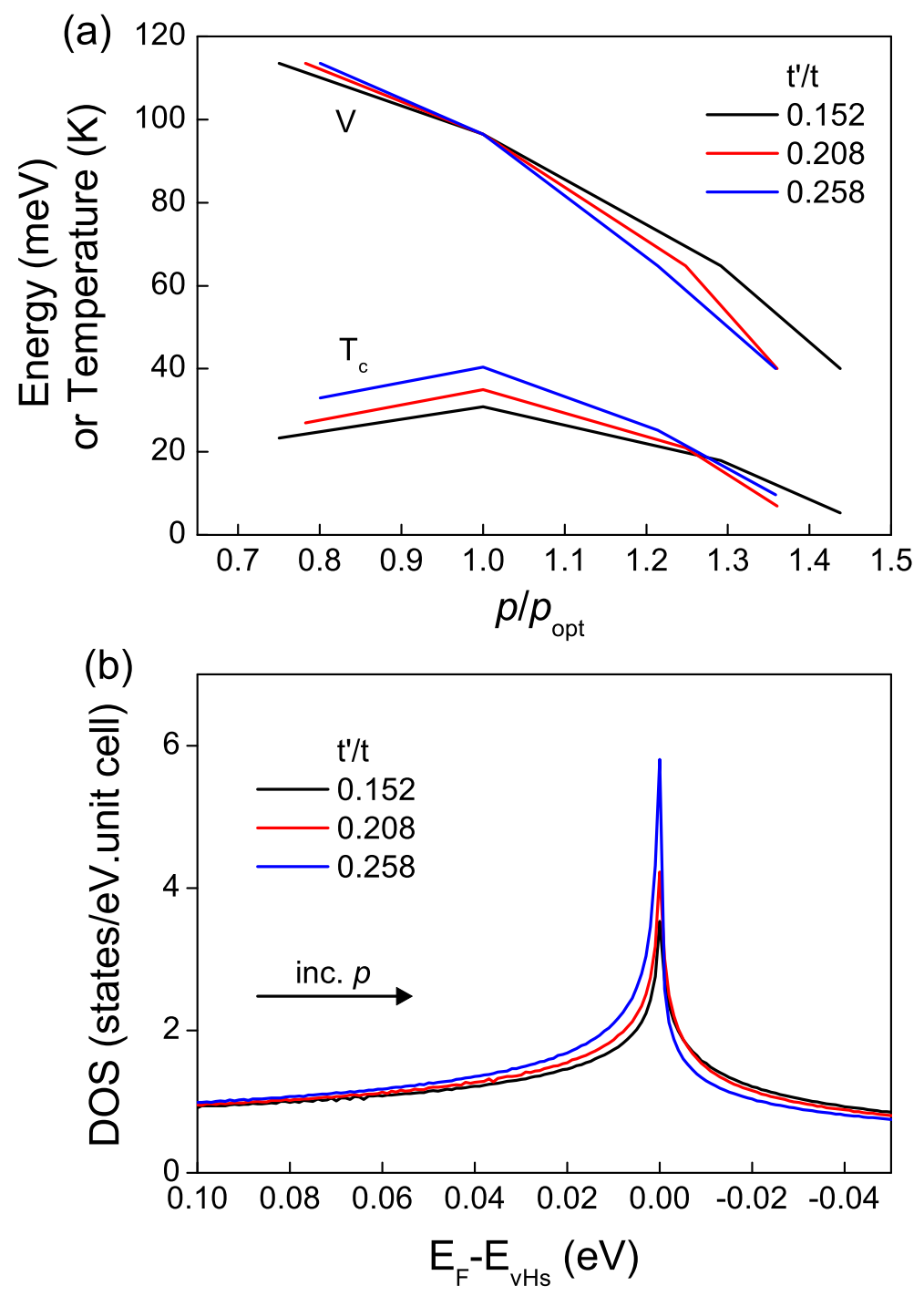

Figure 6.40: (a) Calculated $T_{c}$ phase curve of Bi-2201 for different values of $t^{\prime} / t$. The pairing potential $V$ is a fixed function of $E_{F}-E_{v H s}$. (b) Comparison of the densities of states. 
Assuming the above $V\left(E_{F}-E_{v H s}\right)$ and $\omega_{c}$ values, $T_{c}$ curves were calculated using the bilayer Bi-2212 dispersion (Table 6.1) and an ARPES-derived Tl-2201 dispersion[163]. These curves are plotted in Fig. 6.41 together with the Bi-2201 $T_{c}$ curve as a function of $p / p_{\text {opt }}$. For Bi-2212 the hole concentration was calculated from the average of the bonding and antibonding band Fermi surface areas. Tl-2201 has an experimentally observed $T_{c, \max }$ of about 90K[164], while for Bi-2212 $T_{c, \max }$ has been found to be as high as $96 \mathrm{~K}[165]$. Remarkably, the calculated $T_{c}$ curves display maximal values roughly comparable to these values. The $t^{\prime} / t$ values are 0.208 and 0.417 for the Bi-2201 and Tl-2201 dispersions respectively. Comparison of the calculated densities of states shown in Fig. 6.41(b) again shows that the increases in $T_{c}$ are due to increases in the density of states. In Tl-2201 the dominant increase in DOS appears on the flanks of the vHs while in Bi-2212 the bilayer splitting leads to an overall increase.

When viewed in conjunction with the findings of Pavarini et al. these calculations strongly suggest that $V$ is a universal energy scale in the single-layer cuprates (and possibly the multilayer cuprates), with the variations in $T_{c, \max }$ governed by the exact magnitude of the DOS close to the vHs. In addition, from the observation that the calculated hole concentration increases with $t^{\prime} / t$ we infer that if $V$ is universal, then it is a fixed function of $E_{F}-E_{v H s}$, and not of hole concentration.

A rough extrapolation of the curve in Fig. 6.41(b) gives a value for $V$ at the vHs of about $25 \mathrm{meV}$. Assuming this value results in a calculated $T_{c}$ for Tl-2201 of about 7K. Interestingly the thermoelectric power of a Tl-2201 sample with $T_{c}<4 \mathrm{~K}[146]$ is observed to be negative-linear all the way to 

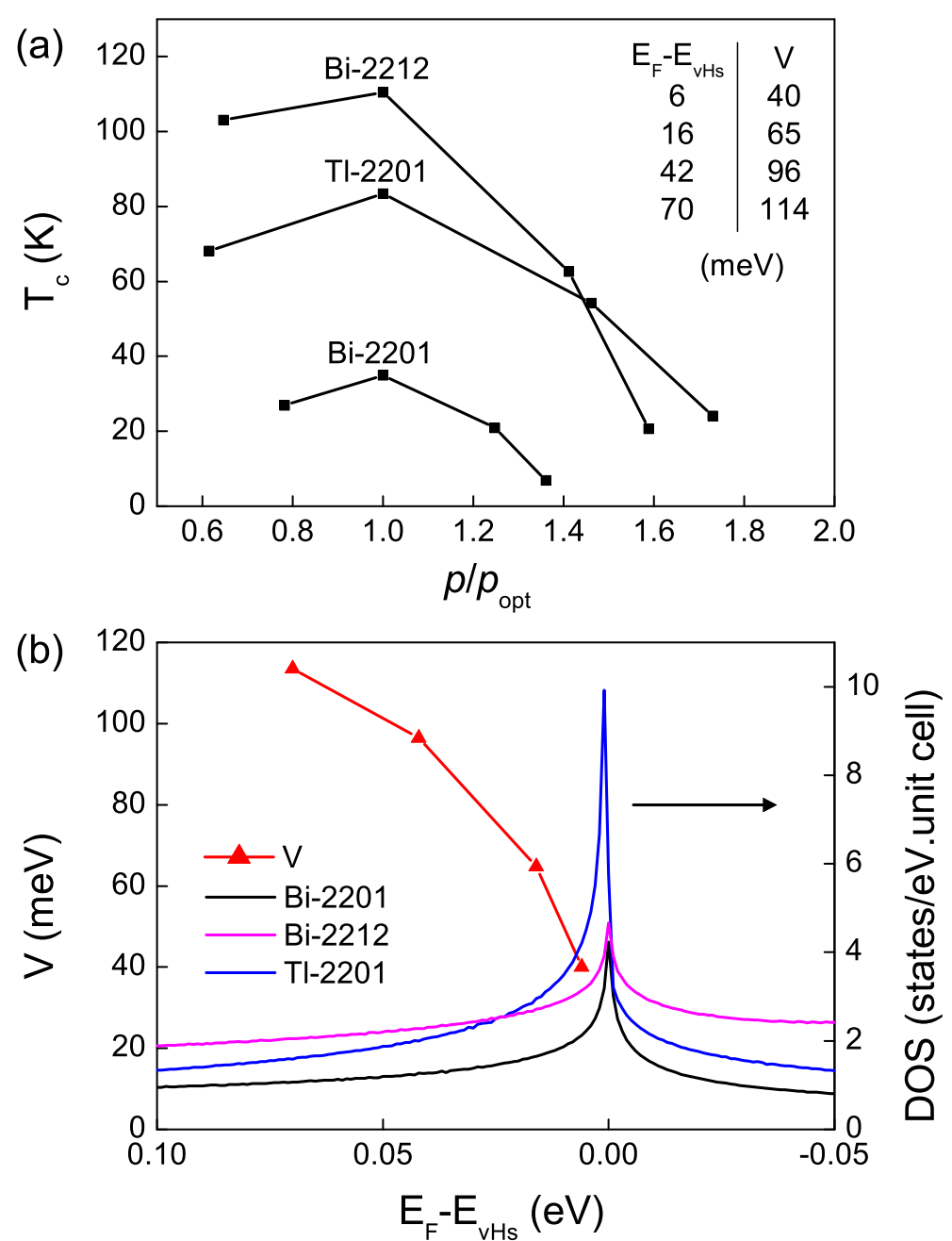

Figure 6.41: (a) Transition temperatures of different cuprates calculated from their respective ARPES dispersions assuming an identical pairing potential as a function of $E_{F}-E_{v H s}$. (b) Comparison of the densities of states and a plot of the pairing potential used in (a). 
about $5 \mathrm{~K}$ providing further confirmation that in Tl-2201 the vHs crossing occurs near the edge of the superconducting dome.

\subsection{Isotope Effect}

In this section the isotope effect is calculated from the energy-momentum dispersion using the model developed in the preceding sections. In section 6.7 it was shown that in order to reproduce the $T_{c}$ phase curve (within the BCS framework), with the Fermi level crossing the vHs in the overdoped region, either the pairing potential amplitude, $V$, or the pairing energy cutoff, $\omega_{c}$, had to increase with decreasing doping. To calculate the isotope effect in $T_{c}$ we assumed the same values for $V$ and $E_{F}-E_{v H s}$ as functions of doping and reduced the pairing potential energy cut-off from $70 \mathrm{meV}$ to $67 \mathrm{meV}$, as suggested by the latest ARPES findings[101] which show a shift in the 'kink' energy of about $3 \mathrm{meV} . T_{c}$ was then recomputed. A plot of $\alpha\left(T_{c}\right)$ versus doping is shown in Fig. 6.42 for Y-123 and Bi-2212 together and compared with La-214 data from Crawford et al.[86].

The calculated isotope effect in Y-123 shows a close resemblance to that observed in La-214. The peak at $p=0.136$ can be explained by examining the energy-resolved contributions, $\zeta(E)$, to the BCS gap equation shown in Fig. 6.43. At this doping the vHs lies $68 \mathrm{meV}$ below $E_{F}$ and so $\zeta(E)$ changes rapidly between the 67 and $70 \mathrm{meV}$ cut-off energies, resulting in the large isotope effect.

The calculations provide an alternative explanation for the peak in the La214 data (generally attributed to spin-charge stripes[89]) but it is important 


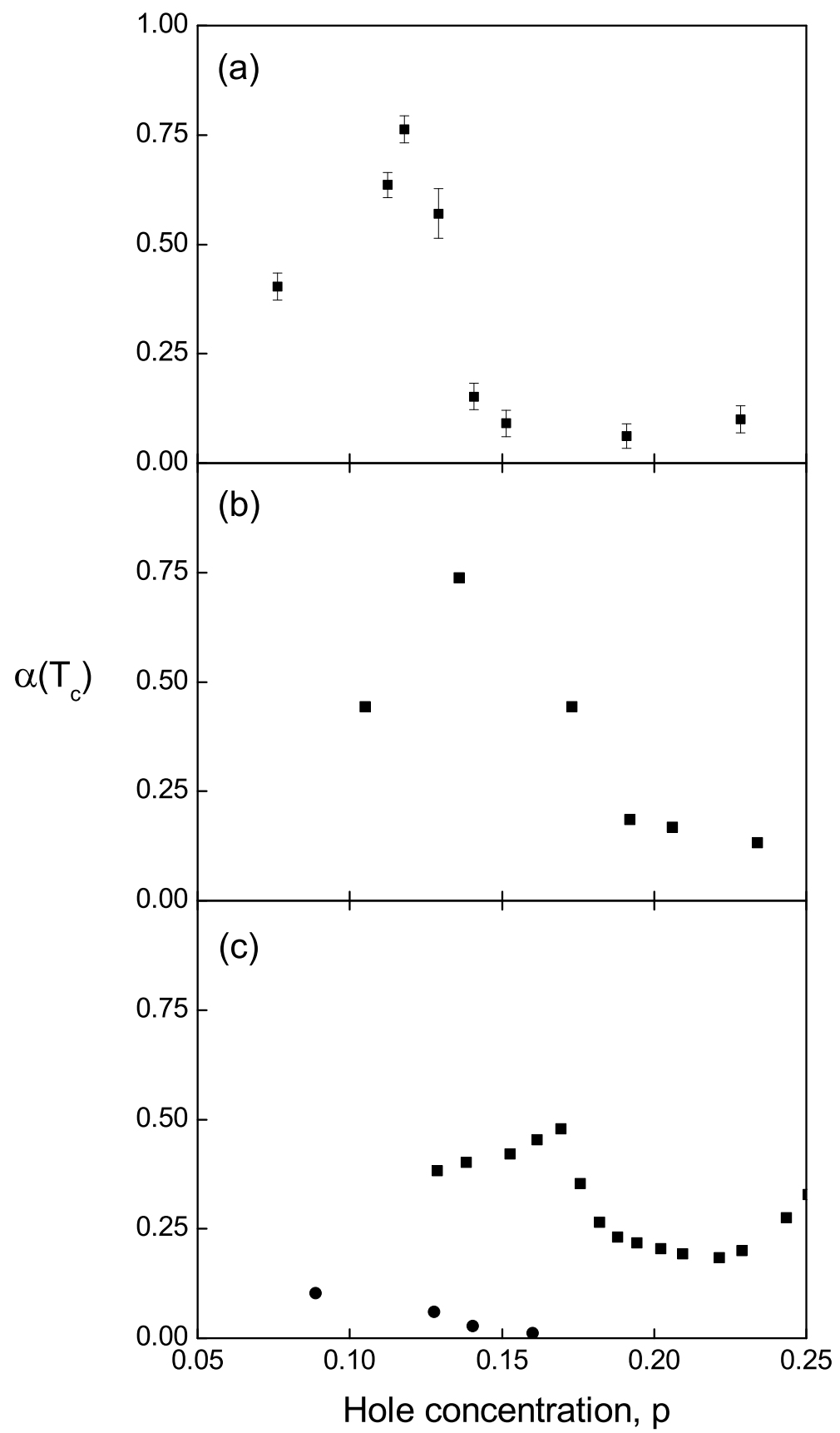

Figure 6.42: (a) Measured oxygen isotope effect in $T_{c}$ of $\mathrm{La}_{2-x} \mathrm{Sr}_{x} \mathrm{CuO}_{4}$ [86]. (b) Calculated isotope effect in $\mathrm{Y}_{0.8} \mathrm{Ca}_{0.2} \mathrm{Ba}_{2} \mathrm{Cu}_{3} \mathrm{O}_{7-\delta}$. (c) Calculated isotope effect in Bi-2212 (squares) and data from Ref. [166] (circles). 


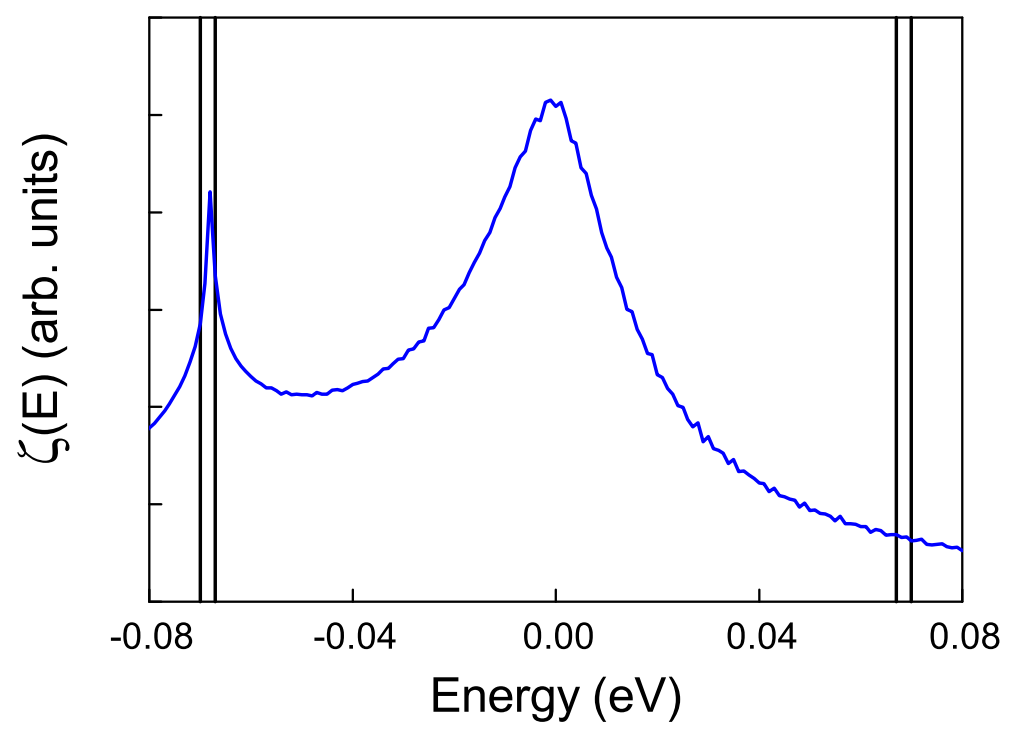

Figure 6.43: Contributions to the summation in the BCS gap equation as a function of energy for Y-123 with $p=0.136$. The vertical lines are placed at $\omega_{c}= \pm 67 \mathrm{meV}$ $\pm 70 \mathrm{meV}$.

to note that this peak seems to be absent in the Y-123 data. As noted in section 2.7 the experimental data shows a steep monotonic rise between $p=0.15$ and 0.05 . The data available is for fully oxygenated samples with varying amounts of non-isovalent cation substitution whereas the calculations have been based on samples with a fixed cation substitution and variable oxygen content. These substituents might play a role in broadening the vHs and thus wiping out the peak at $p=0.136$. We have shown elsewhere[167] that the isotope effect for $\mathrm{Y}-124$ rises rapidly with increasing pressure in this region and could yet signal an effect as shown in Fig. 6.42(b). Turning to Bi2212 , the calculated isotope effect is clearly much larger than that observed in yttrium-doped Bi-2212[166] (circles in Fig. 6.42(c)).

It is important to note that the calculations are based on the assumption 
of a simple doping-independent isotropic isotope shift in the kink energy. Future ARPES results might well reveal further details that need to be included. Details in the pseudogap regime would be especially useful. Pringle et al. [84] explain the doping dependence of $\alpha\left(T_{c}\right)$ with a model in which $\alpha\left(T_{c}\right)$ depends on the ratio $E_{g} / \Delta_{0}$ where $\Delta_{0}^{2}=\Delta_{S C}^{2}+E_{g}^{2}$. The isotope effect in $T_{c}$ increases due to a small isotope effect in the superconducting gap, $\Delta_{S C}$, that is increasingly magnified by the growing pseudogap. The present calculations do not include the pseudogap. However, as shown at the end of section 6.3, the main effect of including the pseudogap in the self-consistent calculation of $\Delta$ (and therefore $T_{c}$ ) is to increase the pairing potential amplitude $V$. One might therefore surmise that the increased $V$ will lead to an increase in $\alpha\left(T_{c}\right)$. Such calculations are yet to be performed. 


\section{Chapter 7}

\section{Summary and Conclusions}

\subsection{Experimental Work}

The aim of the experimental work undertaken here was to determine the oxygen isotope effect in the penetration depth of $\mathrm{YBa}_{2} \mathrm{Cu}_{4} \mathrm{O}_{8}$ along the principal crystallographic axes. The superfluid density is inversely proportional to the square of the penetration depth. Isotope exchanged powders were biaxially aligned in epoxy and AC susceptibility measurements were then performed on these samples. The expected anisotropy in susceptibility and hence the penetration depth was observed, but the magnitude of the low-temperature susceptibilities were such that they resulted in values for the $a b$-plane penetration depths that were over twenty times larger than the generally accepted value.

The value of the penetration depth was found to be extremely sensitive to the upper cut-off radius of the grain size distributions used in the analysis. In an effort to extract some results, upper cut-off radii were selected so that the 
$a b$-plane penetration depths in the ${ }^{16} \mathrm{O}$ and ${ }^{18} \mathrm{O}$ substituted samples matched those reported in the literature. The $b c$ - and $a c$-plane penetration depths were then calculated.

The isotope effect in the $b c$-plane was found to be negligible compared to the $a b$ - and $a c$-planes. Inspection of the phonon modes shows that, with the exception of the $\mathrm{CuO}_{2}$ breathing modes, the motion of the atoms are parallel to the $b c$-plane. The implication is that the electrons prefer to couple to phonon modes in which the atomic motion is perpendicular to the plane of transport. Support for this conjecture comes from site-selective oxygen isotope exchange experiments performed on $\mathrm{Y}_{0.6} \mathrm{Pr}_{0.4} \mathrm{Ba}_{2} \mathrm{Cu}_{3} \mathrm{O}_{7-\delta}[115]$. These experiments showed that the isotope effect in the transition temperature and the superfluid density arises from the oxygen within the superconducting $\mathrm{CuO}_{2}$ planes and not from the apical and chain oxygen.

The isotope effect in the $c$-axis superfluid density was found to be nearly an order of magnitude larger than that expected for a model array of weakly (Josephson) coupled planes. This may reflect the unusual $c$-axis transport in Y-124 which at low temperatures is coherent and is associated with the double $\mathrm{Cu}_{2} \mathrm{O}_{2}$ chains.

\subsection{Computational Work}

The aim of the computational work undertaken here was to calculate various transport and thermodynamic properties of cuprate superconductors from energy-momentum dispersions determined by angle-resolved photoemission spectroscopy (ARPES). All calculations assumed a rigid band approximation. 


\subsubsection{Normal-state Findings}

Using parameters taken directly and only from ARPES the electronic entropy of $\mathrm{Bi}_{2} \mathrm{Sr}_{2} \mathrm{CaCu}_{2} \mathrm{O}_{8+\delta}$ was calculated. ARPES is very much a surface technique. And while the electronic entropy is determined from bulk specific heat measurements, its extraction requires the subtraction of the phonon contribution which can be up to one hundred times larger than the electronic term. For these reasons there exist those who doubt the validity of the data obtained by these techniques. Here an exceptional match with experimental data of Loram et al.[122] was found across the entire T-p phase diagram, thus indirectly confirming both the ARPES and thermodynamic data. Indeed this work provides the first independent verification of the specific heat work of Loram et al.

The temperature and doping dependence of the electronic entropy can be fully explained by the retreat of the Fermi level from a van Hove singularity (vHs) and the opening of a normal state pseudogap. The same can be said for the spin susceptibility as shown by fits made to NMR data. By taking the Fermi level and pseudogap magnitude as fit parameters the electronic entropy was fitted exactly. (Note to the detractors who like to recite the adage "with enough free parameters one can fit anything", these are not free parameters and are tightly constrained by experimental results.) The fits indicate that in Bi-2212 the Fermi level crosses the antibonding band vHs near $p=0.22$ in full agreement with recent ARPES results[42]. The doping evolution of the pseudogap magnitude is consistent with the presence of a quantum critical point near $p=0.19$. 
As mentioned above fits were made to $\mathrm{Y}_{0.8} \mathrm{Ca}_{0.2} \mathrm{Ba}_{2} \mathrm{Cu}_{3} \mathrm{O}_{7-\delta} \mathrm{NMR}$ data using an ARPES derived Y-123 energy-momentum dispersion. The fits indicate that the Fermi level crosses the vHs at the projected edge of the $T_{c}$ dome near $p=0.27$. The presence of a van Hove singularity in the heavily overdoped region appears to be a general feature of the cuprate phase diagram. In the case of $\mathrm{La}_{2-x} \mathrm{Sr}_{x} \mathrm{CuO}_{4}$ the vHs is inferred from ARPES to be crossed in the deeply overdoped region at $p=x=0.23-0.24[59,60]$. A similar situation occurs with $\mathrm{Bi}_{2} \mathrm{Sr}_{2} \mathrm{CuO}_{6}[34]$ and deeply overdoped $\mathrm{Tl}_{2} \mathrm{Ba}_{2} \mathrm{CuO}_{6}$ also lies close to the saddle-point vHs[163]. In the single layer cuprates and double layer cuprates that have large bilayer splitting the crossing of the vHs occurs near where $T_{c}$ goes to zero. In Bi-2212 the bilayer splitting is smaller and the crossing occurs further up the dome when $T_{c}$ is about $60 \mathrm{~K}$.

The diffusion thermoelectric power of overdoped Bi-2212 was calculated under the constant-mean-free-path assumption from the bilayer ARPES dispersion. While the true thermopower signature of the vHs crossing (namely the disappearance of the positive thermopower peak) is obscured by the superconducting state, the calculations show the experimental data to be consistent with the Fermi level crossing the vHs near $T_{c}=60 \mathrm{~K}$. The calculations also reveal the influence of the bonding band vHs in the data as a departure from linearity seen between 100 and 300K.

Underdoped Bi-2212 thermopower data was fitted by including the pseudogap and a model scattering rate with linear energy and temperature dependences. Under the constant-mean-free-path assumption a peak in the energy dependent conductivity occurs at the location of the vHs, $E_{v H s}$. Interestingly it was found that in order to reproduce the peak in the conductivity at $E_{v H s}$ 
the scattering rate had to be proportional to $E-E_{v H s}$ and not $E-E_{F}$. This unusual energy dependence was shown to be a consequence of an underlying momentum dependent scattering rate in which transport in the nodal direction is preferred.

\subsubsection{Superconducting-State Findings}

Some key insights were obtained from comparison of data and calculations in the superconducting state. Among these was the determination of the ground state of the pseudogap, a feature which is shrouded by the superconducting gap. The zero temperature Raman $B_{2 g}$ response was calculated assuming (i) a completely nodal pseudogap ground state, and (ii) a finite Fermi arc pseudogap ground state. In light of these calculations, experimental Raman data demonstrates that there exists a finite Fermi arc at zero temperature (on which the superconducting gap opens), and that the length of this arc decreases as doping decreases.

A finite density of states at the Fermi level resolves the problem how to incorporate a states-non-conserving pseudogap into the self consistent BCS gap equation. In section 6.1 the normal-state entropy was fitted assuming a completely nodal pseudogap. While attempting to calculate the superconducting state entropy data it was found that the pseudogap could not be included in the self consistent calculation of the superconducting gap $\Delta(T)$. Instead $\Delta(T)$ was calculated in the absence of the pseudogap after which it was then re-included. In section 6.3 the most underdoped entropy data was fitted assuming a finite Fermi arc and the pseudogap was then able to be 
successfully incorporated into the self consistent calculation of $\Delta(T)$.

The departure from a pure $d$-wave ' $V$-shaped' gap to a 'U-shaped' gap as observed in ARPES[30, 125] follows naturally from this scenario. The reduced slope of the gap near the nodes is a signature of the smaller superconducting gap opening on the arcs of Fermi surface left ungapped by the larger pseudogap. It is likely that the behaviour of the Fermi arcs can be explained in a fuller treatment that takes into account quasiparticle lifetime effects. For example it has been suggested[123] that both the pseudogap and superconducting gap should be replaced by complex terms of the form $E_{g}(\theta) \rightarrow \frac{E_{g}(\theta)}{1-i \Gamma_{1} / \epsilon(k)}$ and $\Delta(\theta) \rightarrow \frac{\Delta(\theta)}{1-i \Gamma_{0} / \epsilon(k)}$. This naturally leads to a 'Ushaped' gap, and instead of frozen Fermi arcs below $T_{c}$ the Raman data would then insist on a frozen scattering rate $\Gamma_{1}$.

Recently quantum oscillations have been observed in the electrical resistance of $\mathrm{YBa}_{2} \mathrm{Cu}_{3} \mathrm{O}_{6.5}[168](p=0.1)$ and $\mathrm{YBa}_{2} \mathrm{Cu}_{4} \mathrm{O}_{8}[169](p=0.125)$ establishing the existence of a well defined Fermi surface in the ground state of underdoped cuprates. Quantum oscillations are a direct measure of Fermi surface area. The areas observed are consistent with the presence of nodal Fermi pockets that expand as holes are added to the copper oxide planes. Fermi pockets have not been observed by ARPES or predicted by band structure calculations. A suggestion of this author is that perhaps the Fermi pockets represent the area between the bonding and antibonding band Fermi arcs.

By fitting the superconducting state electronic entropy, the doping dependence of the superconducting gap was found to follow the parabolic behaviour of $T_{c}$, while the combined total gap magnitude (pseudogap + superconduct- 
ing gap) increases monotonically with decreasing doping. As mentioned in section 2.6, this two-gap picture has now been observed by many techniques and it signals that the pseudogap arises from a correlation that competes with superconductivity.

Using only the parameters extracted from the entropy fits (namely the doping dependence of the Fermi level, pseudogap and superconducting gap) the doping and temperature dependent superfluid density was calculated. The results show excellent qualitative and quantitative agreement with experimental data. The increasing linearity of the superfluid density with temperature was identified with the approach of the Fermi level to the vHs. The fact that the superfluid density is reproduced with no further parameters provides further confirmation that both the ARPES and specific heat data are valid. This is a highly significant result because it provides the first comprehensive link between these bulk properties and the ARPES measurements which are dominated by the outermost $\mathrm{CuO}_{2}$ layer. Thus, in most respects surface effects do not appear to seriously modify or obscure the band structure which governs bulk properties.

It is a central conclusion of this work that, despite wide variations in bare band structure, the renormalized dispersion near $E_{F}$ seems to lead to a universal phenomenology as seen in the entropy, the susceptibility, the superfluid density, the thermoelectric power and the universal nodal Fermi velocity[98]. This observation calls for theoretical explanation within a strong-coupling picture.

After fitting the superconducting state electronic entropy it was revealed that for a fixed pairing amplitude, $V$, the pairing potential energy cut-off, 
$\omega_{c}$, is linearly related to the distance of the Fermi level from the $\mathrm{vHs}\left(E_{F^{-}}\right.$ $\left.E_{v H s}\right)$. Later it was found that if instead $\omega_{c}$ is kept constant then $V$ is linear in $E_{F^{-}} E_{v H s}$. In section 6.7 explicit calculations of the $T_{c}(p)$ phase curve from the BCS gap equation confirmed that in order for the phase diagram to be consistent with the presence of the vHs in the overdoped regime either $V$ or $\omega_{c}$ has to be a rapidly descending function of $p$. Given the strong doping dependence of high- $T_{c}$ cuprate observables it would probably be more surprising if the pairing interaction was not strongly doping dependent.

It is the interplay between the recession of the Fermi level from the vHs and the increase in $V$ or $\omega_{c}$ with decreasing doping that results in the parabolic dependence of $T_{c}$ and its insensitivity to the presence of the vHs in the overdoped regime. These findings force a revision of all 'van Hove scenario' theories that directly attribute the peak in $T_{c}$ to the van Hove singularity. Of the two possibilities $\left(V\right.$ or $\left.\omega_{c}\right)$ the observation of a doping independent kink energy and the increase in slope of the high energy nodal dispersion with underdoping $[93,98]$ suggests that it is $V$ which varies with doping.

Using $V\left(E_{F}-E_{v H s}\right)$ determined solely from Bi-2201 ARPES data, the $T_{c}$ curves of Tl-2201 and Bi-2212 were calculated from their respective ARPES dispersions. Remarkably the calculated values of $T_{c, \max }$ were found be to comparable with experimental values. When viewed in conjunction with the positive correlation found between $T_{c, \max }$ and the ratio of the next-nearest neighbour to nearest neighbour hopping parameters, $t^{\prime} / t[31]$, the calculations strongly suggest that $V$ is a universal energy scale in the single-layer cuprates (and possibly the multilayer cuprates), with the variations in $T_{c, \max }$ governed 
by the exact magnitude of the density of states close to the vHs. In addition, from the observation that the calculated hole concentration increases with $t^{\prime} / t$ we infer that if $V$ is universal, then it is a fixed function of $E_{F}-E_{v H s}$, and not hole concentration.

With regards to the origin of the pairing interaction, the jury is still out. The magnitude and doping dependence of $V$ is similar to that of the magnetic exchange interaction $J$, suggesting a close relationship between these. The rapid fall in energy scales with doping is also reflected in the pseudogap energy scale and NMR wipe-out temperature. These lines all expand out from the antiferromagnetic phase curve, $T_{N}(p)$, like ripples of remnant magnetic effects suggesting a common magnetic origin for these.

The alternative possibility is phonons. Although a phonon mechanism involving motion of atoms lying outside of the $\mathrm{CuO}_{2}$ plane is likely to be nonuniversal (due to the different bond lengths and substitutionary doping mechanisms in each species of high- $T_{c}$ cuprate) isotope effect experiments show that the modes that affect $T_{c}$ are the $\mathrm{CuO}_{2}$ plane oxygen modes. The $\mathrm{CuO}_{2}$ plane is of course the defining universal feature of the cuprates. The isotope effect experiment carried out as part of this work suggests that it is the transverse vibrational modes that are important. In relation, a large static $\mathrm{CuO}_{2}$ plane buckling (where the $\mathrm{Cu}$ atom is displaced from the plane of the oxygen atoms) is known to reduce $T_{c}[170]$. Newns and Tsuei have recently proposed a fluctuating $\mathrm{Cu}-\mathrm{O}-\mathrm{Cu}$ bond model of high- $T_{c}$ superconductivity[171]. In their model the nonlinear modulation of the $\mathrm{Cu}-\mathrm{Cu}$ bond by planar oxygen vibrations is responsible for Cooper pairing. In light of the present work the major fault of the model is that the peak in $T_{c}$ is ascribed to the vHs. 
Finally, by assuming a small doping-independent $3 \mathrm{meV}$ isotropic shift in $\omega_{c}$, the isotope effect in $T_{c}, \alpha\left(T_{c}\right)$, was calculated. The doping dependence of the calculated $\alpha\left(T_{c}\right)$ is dominated by the density of states and exhibits a peak in the underdoped regime when $E_{F}-E_{v H s}$ becomes comparable to $\omega_{c}$. A peak at a similar doping occurs in the La-214 experimental data but it is most likely due to the presence of static spin/charge stripes at this doping. Such a peak is not visible in the YBCO experimental data. It is hoped that future ARPES experiments will shed further light on the isotope effects in the energy-momentum dispersion so that the agreement between the data and calculations may be improved. 


\section{Bibliography}

[1] H. Kamerlingh Onnes. Further experiments with liquid helium. D. On the change of electrical resistance of pure metals at very low temperatures, etc. V. The disappearance of the resistance of mercury. KNAW Proc., 14(1):113-115, 1911.

[2] H. Kamerlingh Onnes. Further experiments with liquid helium. G. On the change of electrical resistance of pure metals etc. VI. On the sudden change in the rate at which the resistance of mercury disappears. KNAW Proc., 14(2):818-821, 1911-1912.

[3] J. Bardeen, L. N. Cooper, and J. R. Schrieffer. Theory of superconductivity. Physical Review, 108(5):1175-1204, 1957.

[4] G. Baym. G1:3 BCS - from atoms and nuclei to the cosmos. In March Meeting 2007 Program and Show Guide, page 174, 2007.

[5] A. Bohr, B. R. Mottelson, and D. Pines. Possible analogy between the excitation spectra of nuclei and those of the superconducting metallic state. Phys. Rev., 110(4):936-938, 1958. 
[6] A. Fabrocini, S. Fantoni, A. Y. Illarionov, and K. E. Schmidt. ${ }^{1} S_{0}$ superfluid phase transition in neutron matter with realistic nuclear potentials and modern many-body theories. Phys. Rev. Lett., 95:192501, 2005.

[7] K. Rajagopal and F. Wilczek. The condensed matter physics of QCD. arXiv:hep-ph/0011333, 2000.

[8] J. G. Bednorz and K. A. Müller. Possible high- $T_{c}$ superconductivity in the Ba-La-Cu-O system. Z. Phys. B, 64:189-193, 1986.

[9] K. A. Müller and J. G. Bednorz. The discovery of a class of hightemperature superconductors. Science, 237:1133-1139, 1987.

[10] H. Takagi, S. Uchida, K. Kitazawa, and S. Tanaka. High- $T_{c}$ superconductivity of La-Ba-Cu oxides. II. - specification of the superconducting phase. Jpn. J. Appl. Phys., 26:L123-L124, 1987.

[11] M. K. Wu, J. R. Ashburn, C. J. Torng, P. H. Hor, R. L. Meng, L. Gao, Z. J. Huang, Y. Q. Wang, and C. W. Chu. Superconductivity at $93 \mathrm{~K}$ in a new mixed-phase $\mathrm{Y}-\mathrm{Ba}-\mathrm{Cu}-\mathrm{O}$ compound system at ambient pressure. Phys. Rev. Lett., 58:908-910, 1987.

[12] P. H. Hor, R. L. Meng, Y. Q. Wang, L. Gao, Z. J. Huang, J. Bechtold, K. Forster, and C. W. Chu. Superconductivity above 90K in the squareplanar compound system $\mathrm{ABa}_{2} \mathrm{Cu}_{3} \mathrm{O}_{6+x}$ with $\mathrm{A}=\mathrm{Ym} \mathrm{La}, \mathrm{Nd}, \mathrm{Sm}, \mathrm{Eu}$, Gd, Ho, Er and Lu. Phys. Rev. Lett., 58:1891-1894, 1987. 
[13] C. W. Chu, L. Gao, F. Chen, Z. J. Huang, R. L. Meng, and Y. Y. Xue. Superconductivity above $150 \mathrm{~K}$ in $\mathrm{HgBa}_{2} \mathrm{Ca}_{2} \mathrm{Cu}_{3} \mathrm{O}_{8+\delta}$ at high pressure. Nature, 365:323-325, 1993.

[14] W. D. Allen, R. H. Dawton, J. M. Lock, A. B. Pippard, and D. Shoenberg. Superconductivity of tin isotopes. Nature, 166:1071-1072, 1950.

[15] H. Kotegawa, Y. Tokunaga, K. Ishida, G. Q. Zheng, Y. Kitaoka, H. Kito, A. Iyo, K. Tokiwa, T. Watanabe, and H. Ihara. Unusual magnetic and superconducting characteristics in multilayered high- $T_{c}$ cuprates: ${ }^{63} \mathrm{Cu}$ NMR study. Phys. Rev. B, 64:064515, 2001.

[16] G. Burns. High-temperature superconductivity an introduction. Academic Press, Inc., 1992.

[17] R. M. Hazen. Crystal structures of high-temperature superconductors. In D. M. Ginsberg, editor, Physical properties of high temperature superconductors., volume 2, chapter 3. World scientific, Singapore, 1990.

[18] M. R. Presland, J. L. Tallon, R. G. Buckley, R. S. Liu, and N. E. Flower. General trends in oxygen stoichiometry effects on $T_{c}$ in $\mathrm{Bi}$ and Tl superconductors. Physica C, 176:95-105, 1991.

[19] C. M. Varma, P. B. Littlewood, S. Schmitt-Rink, E. Abrahams, and A. E. Ruckenstein. Phenomenology of the normal state of $\mathrm{Cu}-\mathrm{O}$ hightemperature superconductors. Phys. Rev. Lett., 63:1996-1999, 1989.

[20] J. D. Koralek, J. F. Douglas, N. C. Plumb, Z. Sun, A. V. Fedorov, M. M. Murnane, H. C. Kapteyn, S. T. Cundiff, Y. Aiura, K. Oka, 
H. Eisaki, and D. S. Dessau. Laser based angle-resolved photoemission, the sudden approximation, and quasiparticle-like spectral peaks in $\mathrm{Bi}_{2} \mathrm{Sr}_{2} \mathrm{CaCu}_{2} \mathrm{O}_{8+\delta}$. Phys. Rev. Lett., 96:017005, 2006.

[21] P. Aebi, J. Osterwalder, P. Schwaller, Schlapbach L., M. Shimoda, T. Mochiku, and K. Kadowaki. Complete Fermi surface mapping of $\mathrm{Bi}_{2} \mathrm{Sr}_{2} \mathrm{CaCu}_{2} \mathrm{O}_{8+x}$ (001): coexistence of short range antiferromagnetic correlations and metallicity in the same phase. Phys. Rev. Lett., 72:2757-2761, 1994.

[22] D. M. King, Z. X. Shen, D. S. Dessau, D. S. Marshall, C. H. Park, W. E. Spicer, J. L. Peng, Z. Y. Li, and R. L. Greene. Obervation of a saddlepoint singularity in $\mathrm{Bi}_{2}\left(\mathrm{Sr}_{0.97} \mathrm{Pr}_{0.03}\right)_{2} \mathrm{CuO}_{6+\delta}$ and its implications for normal and SC state properties. Phys. Rev. Lett., 73:3298-3301, 1994.

[23] J. Ma, C. Quitmann, R. J. Kelley, P. Alméras, H. Berger, G. Margaritondo, and M. Onellion. Observation of a Van Hove singularity in $\mathrm{Bi}_{2} \mathrm{Sr}_{2} \mathrm{CaCu}_{2} \mathrm{O}_{8+x}$ with angle-resolved photoemission. Phys. Rev. B, 51:3832-3389, 1995.

[24] A. G. Loeser, Z. X. Shen, D. S. Dessau, D. S. Marshall, C. H. Park, P. Fournier, and A. Kapitulnik. Excitation gap in the normal state of underdoped $\mathrm{Bi}_{2} \mathrm{Sr}_{2} \mathrm{CaCu}_{2} \mathrm{O}_{8+\delta}$. Science, 273:325-329, 1996.

[25] H. Ding, T. Yokoya, J. C. Campuzano, T. Takahashi, M. Randeria, M. R. Norman, T. Mochiku, K. Kadowaki, and J. Giapintzakis. Spectroscopic evidence for a pseudogap in the normal state of underdoped high- $T_{c}$ superconductors. Nature, 382:51-54, 1996. 
[26] M. R. Norman, H. Ding, M. Randeria, J. C. Campuzano, T. Yokoya, T. Takeuchi, T. Takahashi, T. Mochiku, K. Kadowaki, P. Guptasarma, and D. G. Hinks. Destruction of the fermi surface in underdoped high$T_{c}$ superconductors. Nature, 392:157-160, 1998.

[27] B. O. Wells, Z. X. Shen, D. S. Dessau, W. E. Spicer, D. B. Mitzi, L. Lombardo, A. Kapitulnik, and A. J. Arko. Evidence for k-dependent, in-plane anisotropy of the superconducting gap in $\mathrm{Bi}_{2} \mathrm{Sr}_{2} \mathrm{CaCu}_{2} \mathrm{O}_{8+\delta}$. Phys. Rev. B, 46:11830-11834, 1992.

[28] Z. X. Shen, D. S. Dessau, B. O. Wells, D. M. King, W. E. Spicer, A. J. Arko, D. Marshall, L. W. Lombardo, A. Kapitulnik, P. Dickinson, S. Doniach, J. DiCarlo, A. G. Loeser, and C. H. Park. Anomalously large gap anisotropy in the $a-b$ plane of $\mathrm{Bi}_{2} \mathrm{Sr}_{2} \mathrm{CaCu}_{2} \mathrm{O}_{8+\delta}$. Phys. Rev. Lett., 70:1553-1556, 1993.

[29] H. Ding, M. R. Norman, J. C. Campuzano, M. Randeria, A. F. Bellman, T. Yokoya, T. Takahashi, T. Mochiku, and K. Kadowaki. Angle-resolved photoemission spectroscopy of the superconducting gap anisotropy in $\mathrm{Bi}_{2} \mathrm{Sr}_{2} \mathrm{CaCu}_{2} \mathrm{O}_{8+x}$. Phys. Rev. B, 54:R9678-R9681, 1996.

[30] J. Mesot, M. R. Norman, H. Ding, M. Randeria, J. C. Campuzano, A. Paramekanti, H. M. Fretwell, A. Kaminski, T. Takeuchi, T. Yokoya, T. Sato, T. Takehashi, T. Mochiku, and K. Kadowaki. Superconducting gap anisotropy and quasiparticle interactions: A doping dependent photoemission study. Phys. Rev. Lett., 83:840-843, 1999. 
[31] E. Pavarini, I. Dasgupta, T. Saha-Dasgupta, O. Jepsen, and O. K. Andersen. Band-structure trend in hole-doped cuprates and correlation with $T_{\text {cmax }}$. Phys. Rev. Lett., 87:047003, 2001.

[32] M. R. Norman, M. Randeria, H. Ding, and J. C. Campuzano. Phenomenological models for the gap anisotropy of $\mathrm{Bi}_{2} \mathrm{Sr}_{2} \mathrm{CaCu}_{2} \mathrm{O}_{8}$ as measured by angle-resolved photoemission spectroscopy. Phys. Rev. $B, 52: 615-622,1995$.

[33] C. Kittel. Introduction to solid state physics. John Wiley \& Sons Inc., New York, 6 edition, 1986.

[34] T. Kondo, T. Takeuchi, T. Yokoya, S. Tsuda, S. Shin, and U. Mizutani. Hole-concentration dependence of band structure in $(\mathrm{Bi}, \mathrm{Pb})_{2}(\mathrm{Sr}, \mathrm{La})_{2} \mathrm{CuO}_{6+\delta}$ determined by the angle-resolved photoemission spectroscopy. J. Electron. Spectrosc. Relat. Phenom., 137140:663-668, 2004.

[35] S. Massidda, J. Yu, and A. J. Freeman. Electronic structure and properties of $\mathrm{Bi}_{2} \mathrm{Sr}_{2} \mathrm{CaCu}_{2} \mathrm{O}_{8}$, the third high- $T_{c}$ superconductor. Physica $C$, 152:251-258, 1988.

[36] O. K. Andersen, A. I. Liechtenstein, O. Jepsen, and F. Paulsen. LDA energy bands, low-energy hamiltonians, $t^{\prime}, t^{\prime \prime}, t_{\perp}(\mathbf{k})$ and $J_{\perp}$. J. Phys. Chem. Solids, 56:1573-1591, 1995.

[37] A. I. Liechtenstein, O. Gunnarsson, O. K. Andersen, and R. M. Martin. Quasiparticle bands and superconductivity in bilayer cuprates. Phys. Rev. B, 54:12505-12508, 1996. 
[38] D. L. Feng, N. P. Armitage, D. H. Lu, A. Damascelli, J. P. Hu, P. Bogdanov, A. Lanzara, F. Ronning, K. M. Shen, H. Eisaki, C. Kim, Z. X. Shen, J. I. Shimoyama, and K. Kishio. Bilayer splitting in the electronic structure of heavily overdoped $\mathrm{Bi}_{2} \mathrm{Sr}_{2} \mathrm{CaCu}_{2} \mathrm{O}_{8+\delta}$. Phys. Rev. Lett, 86:5550-5553, 2001.

[39] Y. D. Chuang, A. D. Gromko, A. Fedorov, Y. Aiura, K. Oka, Y. Ando, H. Eisaki, S. I. Uchida, and D. S. Dessau. Doubling of the bands in overdoped $\mathrm{Bi}_{2} \mathrm{Sr}_{2} \mathrm{CaCu}_{2} \mathrm{O}_{8+\delta}$ : evidence for $c$-axis bilayer coupling. Phys. Rev. Lett, 87:117002, 2001.

[40] H. Ding, A. F. Bellman, J. C. Campuzano, M. Randeria, M. R. Norman, T. Yokoya, T. Takahashi, H. Katayama-Yoshida, T. Mochiku, K. Kadowaki, G. Jennings, and G. P. Brivio. Electronic excitations in $\mathrm{Bi}_{2} \mathrm{Sr}_{2} \mathrm{CaCu}_{2} \mathrm{O}_{8}$ : Fermi surface, dispersion and absence of bilayer splitting. Phys. Rev. Lett, 76:1533-1536, 1996.

[41] Y. D. Chuang, A. D. Gromko, D. S. Dessau, Y. Aiura, Y. Yamaguchi, K. Oka, A. J. Arko, J. Joyce, H. Eisaki, S. I. Uchida, K. Nakamura, and Y. Ando. Reexamination of the electronic structure of $\mathrm{Bi}_{2} \mathrm{Sr}_{2} \mathrm{CaCu}_{2} \mathrm{O}_{8+\delta}$ and $\mathrm{Bi}_{2} \mathrm{Sr}_{2} \mathrm{CuO}_{6+\delta}$ : electronlike portions of the Fermi surface and depletion of spectral weight near $\bar{M}$. Phys. Rev. Lett, 83:3717-3720, 1999.

[42] A. Kaminski, S. Rosenkranz, H. M. Fretwell, M. R. Norman, M. Randeria, J. C. Campuzano, J. M. Park, Z. Z. Li, and H. Raffy. Change of 
Fermi-surface topology in $\mathrm{Bi}_{2} \mathrm{Sr}_{2} \mathrm{CaCu}_{2} \mathrm{O}_{8+\delta}$ with doping. Phys. Rev. $B, 73: 174511,2006$.

[43] T. Xiang and J. M. Wheatley. c Axis superfluid response of copper oxide superconductors. Phys. Rev. Lett., 77:4632-4635, 1996.

[44] A. C. Rose-Innes and E. H. Rhoderick. Introduction to superconductivity. Pergamon Press, Oxford, England, second edition, 1978.

[45] W. N. Hardy, D. A. Bonn, D. C. Morgan, R. Liang, and K. Zhang. Precision measurements of the temperature dependence of $\lambda$ in $\mathrm{YBa}_{2} \mathrm{Cu}_{3} \mathrm{O}_{6.95}$ : strong evidence for nodes in the gap function. Phys. Rev. Lett., 70:3999-4002, 1993.

[46] T. P. Devereaux, D. Einzel, B. Stadlober, R. Hackl, D. H. Leach, and J. J. Neumeier. Electronic Raman scattering in high- $T_{c}$ superconductors: a probe of $d_{x^{2}-y^{2}}$ pairing. Phys. Rev. Lett., 72:396-399, 1994.

[47] N. Bulut and D. J. Scalapino. Analysis of NMR data in the superconducting state of $\mathrm{YBa}_{2} \mathrm{Cu}_{3} \mathrm{O}_{7}$. Phys. Rev. Lett., 68:706-709, 1992.

[48] C. C. Tsuei and J. R. Kirtley. Phase-sensitive tests of pairing symmetry in cuprate superconductors. Physica C, 282-287:4-11, 1997.

[49] C. C. Tsuei and J. R. Kirtley. Pairing symmetry in cuprate superconductors. Rev. Mod. Phys., 72:969-1016, 2000.

[50] D. S. Dessau, Z. X. Shen, D. M. King, D. S. Marshall, L. W. Lombardo, P. H. Dickinson, A. G. Loeser, J. DiCarlo, C. H. Park, A. Kapitulnik, and W. E. Spicer. Key features in the measured band structure of 
$\mathrm{Bi}_{2} \mathrm{Sr}_{2} \mathrm{CaCu}_{2} \mathrm{O}_{8+\delta}$ : flat bands at $E_{F}$ and Fermi surface nesting. Phys. Rev. Lett., 71:2781-2784, 1993.

[51] R. S. Markiewicz. A survey of the van hove scenario for high- $T_{c}$ superconductivity with special emphasis on pseudogaps and striped phases. J. Phys. Chem. Solids, 58:1179-1310, 1997.

[52] D. M. Newns, C. C. Tsuei, and P. C. Pattnaik. Van Hove scenario for $d$-wave superconductivity in cuprates. Phys. Rev. B, 52:13611-13618, 1995.

[53] C. C. Tsuei, D. M. Newns, C. C. Chi, and P. C. Pattnaik. Anomalous isotope effect and Van Hove singularity in superconducting $\mathrm{Cu}$ oxides. Phys. Rev. Lett., 65:2724-2727, 1990.

[54] D. M. Newns, C. C. Tsuei, R. P. Huebener, P. J. M. van Bentum, P. C. Pattnaik, and C. C. Chi. Quasiclassical transport at a van Hove singularity in cuprate superconductors. Phys. Rev. Lett., 73:1695-1698, 1994.

[55] J. Bouvier and J. Bok. Gap anisotropy and van Hove singularities in high- $T_{c}$ superconductors. Physica C, 249:117-122, 1995.

[56] D. M. Newns, H. R. Krishnamurthy, P. C. Pattnaik, C. C. Tsuei, and C. L. Kane. Saddle-point pairing: an electronic mechanism for superconductivity. Phys. Rev. Lett., 69:1264-1267, 1992. 
[57] T. Kondo, T. Takeuchi, U. Mizutani, T. Yokoya, S. Tsuda, and S. Shin. Contribution of electronic structure to thermoelectric power in $(\mathrm{Bi}, \mathrm{Pb})_{2}(\mathrm{Sr}, \mathrm{La})_{2} \mathrm{CuO}_{6+\delta}$. Phys. Rev. B, 72:024533, 2005.

[58] A. Ino, C. Kim, T. Mizokawa, Z. X. Shen, A. Fujimori, M. Takaba, K. Tamasaku, H. Eisaki, and S. Uchida. Fermi surface and band dispersion in $\mathrm{La}_{2-x} \mathrm{Sr}_{x} \mathrm{CuO}_{4}$. J. Phys. Soc. Jpn., 68:1496-1499, 1999.

[59] A. Ino, C. Kim, M. Nakamura, T. Yoshida, T. Mizokawa, A. Fujimori, Z. X. Shen, T. Takeshita, H. Eisaki, and S. Uchida. Doping dependent evolution of the electronic structure of $\mathrm{La}_{2-x} \mathrm{Sr}_{x} \mathrm{CuO}_{4}$ in the superconducting and metallic phases. Phys. Rev. B, 65:094504, 2002.

[60] T. Yoshida, X. J. Zhou, K. Tananka, W. L. Yang, Z. Hussain, Z. X. Shen, A. Fujimori, S. Sahrakorpi, M. Lindroos, R. S. Markiewicz, A. Bansil, S. Komiya, Y. Ando, H. Eisaki, T. Kakeshita, and S. Uchida. Systematic doping evolution of the underlying Fermi surface of $\mathrm{La}_{2-x} \mathrm{Sr}_{x} \mathrm{CuO}_{4}$. Phys. Rev. B, 74:224510, 2006.

[61] T. Yoshida, X. J. Zhou, D. H. Lu, S. Komiya, Y. Ando, H. Eisaki, T. Kakeshita, S. Uchida, Z. Hussain, Z. X. Shen, and A. Fujimori. Low-energy electronic structure of the high- $T_{c}$ cuprates $\mathrm{La}_{2-x} \mathrm{Sr}_{x} \mathrm{CuO}_{4}$ studied by angle-resolved photoemission spectroscopy. J. Phys. Condens. Matter, 19:125209, 2007.

[62] T. Timusk and B. Statt. The pseudogap in high-temperature superconductors: an experimental survey. Rep. Prog. Phys., 62:61-122, 1999. 
[63] M. R. Norman, D. Pines, and C. Kallin. The pseudogap: friend or foe of high $T_{c}$ ? Adv. Phys., 54:715-733, 2005.

[64] W. W. Warren, Jr, R. E. Walstedt, G. F. Brennert, R. J. Cava, R. Tycko, R. F. Bell, and G. Dabbagh. Cu spin dynamics and superconducting precursor effects in planes above $T_{c}$ in $\mathrm{YBa}_{2} \mathrm{Cu}_{3} \mathrm{O}_{6.7}$. Phys. Rev. Lett., 62:1193-1196, 1989.

[65] D. C. Johnston. Magnetic susceptibility scaling in $\mathrm{La}_{2-x} \mathrm{Sr}_{x} \mathrm{CuO}_{4-y}$. Phys. Rev. Lett., 62:957-960, 1989.

[66] H. Alloul, T. Ohno, and P. Mendels. ${ }^{89} \mathrm{Y}$ NMR evidence for a Fermiliquid behaviour in $\mathrm{YBa}_{2} \mathrm{Cu}_{3} \mathrm{O}_{6+\delta}$. Phys. Rev. Lett., 63:1700-1703, 1989.

[67] J. W. Loram, K. A. Mirza, J. R. Cooper, W. Y. Liang, and J. M. Wade. Electronic specific heat of $\mathrm{YBa}_{2} \mathrm{Cu}_{3} \mathrm{O}_{6+x}$ from 1.8 to $300 \mathrm{~K}$. J. Supercon., 7:243-249, 1994.

[68] J. W. Loram, K. A. Mirza, J. M. Wade, J. R. Cooper, and W. Y. Liang. The electronic specific heat of cuprate superconductors. Physica $C$, 235-240:134-137, 1994.

[69] H. J. Tao, F. Lu, and E. L. Wolf. Observation of pseudogap in $\mathrm{Bi}_{2} \mathrm{Sr}_{2} \mathrm{CaCu}_{2} \mathrm{O}_{8+\delta}$ single crystals with electron tunneling spectroscopy. Physica C, 282-287:1507-1508, 1997. 
[70] Ch. Renner, B. Revaz, J. Y. Genoud, K. Kadowaki, and O. Fischer. Pseudogap precursor of the superconducting gap in under- and overdoped $\mathrm{Bi}_{2} \mathrm{Sr}_{2} \mathrm{CaCu}_{2} \mathrm{O}_{8+\delta}$. Phys. Rev. Lett., 80:149-152, 1998.

[71] A. Matsuda, S. Sugita, and T. Watanabe. Temperature and doping dependence of the $\mathrm{Bi}_{2.1} \mathrm{Sr}_{1.9} \mathrm{CaCu}_{2} \mathrm{O}_{8+\delta}$ pseudogap and superconducting gap. Phys. Rev. B, 60:1377-1381, 1999.

[72] A. Kanigel, M. R. Norman, M. Randeria, U. Chatterjee, S. Souma, A. Kaminski, H. M. Fretwell, S. Rosenkranz, M. Shi, T. Sato, T. Takahashi, Z. Z. Li, H. Raffy, K. Kadowaki, D. Hinks, L. Ozyuzer, and J. C. Campuzano. Evolution of the pseudogap from Fermi arcs to the nodal liquid. Nature Physics, 2:447-451, 2006.

[73] M. R. Norman, M. Randeria, H. Ding, and J. C. Campuzano. Phenomenology of the low-energy spectral function in high- $T_{c}$ superconductors. Phys. Rev. B, 57:R11093-R11096, 1998.

[74] J. L. Tallon, G. V. M. Williams, and J. W. Loram. NMR studies on HTS cuprates: re-assessing the models. J. Phys. Chem. Solids, 59:2145-2149, 1998.

[75] J. L. Tallon, G. V. M. Williams, and J. W. Loram. Factors affecting the optimal design of high- $T_{c}$ superconductors - the pseudogap and critical doping. Physica C, 338:9-17, 2000.

[76] G. Deutscher. Coherence and single-particle excitations in the hightemperature superconductors. Nature, 397:410-412, 1999. 
[77] M. Le Tacon, A. Sacuto, A. Georges, G. Kotliar, Y. Gallais, D. Colson, and A. Forget. Two energy scales and two distinct quasiparticle dynamics in the superconducting state of underdoped cuprates. Nature Physics, 2:537-543, 2006.

[78] K. Tanaka, W. S. Lee, D. H. Lu, A. Fujimori, T. Fujii, Risdiana, I. Terasaki, D. J. Scalapino, T. P. Devereaux, Z. Hussain, and X. Shen, Z. Distinct Fermi-momentum-dependent energy gaps in deeply underdoped Bi2212. Science, 314:1910-1913, 2006.

[79] T. Kondo, T. Takeuchi, A. Kaminski, S. Tsuda, and S. Shin. Evidence for two energy scales in the superconducting state of optimally doped $(\mathrm{Bi}, \mathrm{Pb})_{2}(\mathrm{Sr}, \mathrm{La})_{2} \mathrm{CuO}_{6+\delta}$. Phys. Rev. Lett., 98:267004, 2007.

[80] M. C. Boyer, W. D. Wise, K. Chatterjee, M. Yi, T. Kondo, T. Takeuchi, H. Ikuta, and E. W. Hudson. Imaging the two gaps of the hightemperature superconductor $\mathrm{Bi}_{2} \mathrm{Sr}_{2} \mathrm{CuO}_{6+x}$. Nature Physics, 3:802$806,2007$.

[81] J. L. Tallon, G. V. M. Willams, M. P. Staines, and C. Bernhard. Energy and length scales in the superconducting phase diagram for HTSC cuprates. Physica C, 235-240:1821-1822, 1994.

[82] N. Mannella, W. L. Yang, X. J. Zhou, H. Zheng, J. F. Mitchell, J. Zaanen, T. P. Devereaux, N. Nagaosa, Z. Hussain, and Z. X. Shen. Nodal quasiparticle in pseudogapped colossal magnetoresistive manganites. Nature, 438:474-478, 2005. 
[83] J. P. Franck, J. Jung, M. A-K. Mohamed, S. Gygax, and G. I. Sproule. Observation of an oxygen isotope effect in superconducting $\left(\mathrm{Y}_{1-x} \mathrm{Pr}_{x}\right) \mathrm{Ba}_{2} \mathrm{Cu}_{3} \mathrm{O}_{7-\delta}$. Phys. Rev. B, 44:5318-5321, 1991.

[84] D. J. Pringle, G. V. M. Williams, and J. L. Tallon. Effect of doping and impurities on the oxygen isotope effect in high-temperature superconducting cuprates. Phys. Rev. B, 62:12527-12533, 2000.

[85] M. K. Crawford, M. N. Kunchur, W. E. Farneth, E. M. McCarron, III, and S. J. Poon. Anomalous oxygen isotope effect in $\mathrm{La}_{2-x} \mathrm{Sr}_{x} \mathrm{CuO}_{4}$. Phys. Rev. B, 41:282-287, 1990.

[86] M. K. Crawford, W. E. Farneth, E. M. McCarron III, R. L. Harlow, and A. H. Moudden. Oxygen isotope effect and structural phase transitions in $\mathrm{La}_{2} \mathrm{CuO}_{4}$-based superconductors. Science, 250:1390-1394, 1990.

[87] J. L. Tallon, R. S. Islam, J. Storey, G. V. M. Williams, and J. R. Cooper. Isotope effect in the superfluid density of high temperature superconducting cuprates: stripes, pseudogap and impurities. Phys. Rev. Lett., 94:237002, 2005.

[88] R. S. Islam. Influence of hole content and controlled disorder on the magnetic susceptibility, isotope exponents and resistivity of high- $T_{c}$ cuprates. PhD thesis, U. of Cambridge, England, 2005.

[89] J. M. Tranquada, B. J. Sternlieb, J. D. Axe, Y. Nakamura, and S. Uchida. Evidence for stripe correlations of spins and holes in copper oxide superconductors. Nature, 375:561-563, 1995. 
[90] N. W. Aschcroft and N. D. Mermin. Solid State Physics., chapter 26, pages 519-521. Saunders College, Philadelphia, 1976.

[91] P. V. Bogdanov, A. Lanzara, S. A. Kellar, X. J. Zhou, E. D. Lu, W. J. Zheng, G. Gu, J. I. Shimoyama, K. Kishio, H. Ikeda, R. Yoshizaki, Z. Hussain, and Z. X. Shen. Evidence for an energy scale for quasiparticle dispersion in $\mathrm{Bi}_{2} \mathrm{Sr}_{2} \mathrm{CaCu}_{2} \mathrm{O}_{8}$. Phys. Rev. Lett., 85:2581-2584, 2000 .

[92] A. Kaminski, M. Randeria, J. C. Campuzano, M. R. Norman, H. Fretwell, J. Mesot, T. Sato, T. Takahashi, and K. Kadowaki. Renormalization of spectral line shape and dispersion below $T_{c}$ in $\mathrm{Bi}_{2} \mathrm{Sr}_{2} \mathrm{CaCu}_{2} \mathrm{O}_{8+\delta}$. Phys. Rev. Lett., 86:1070-1073, 2001.

[93] A. Lanzara, P. V. Bogdanov, X. J. Zhou, S. A. Kellar, D. L. Feng, E. D. Lu, T. Yoshida, H. Eisaki, A. Fujimori, K. Kishio, J. I. Shimoyama, T. Noda, S. Uchida, Z. Hussain, and Z. X. Shen. Evidence for ubiquitous strong electron-phonon coupling in high-temperature superconductors. Nature, 412:510-514, 2001.

[94] P. D. Johnson, T. Valla, A. V. Fedorov, Z. Yusof, B. O. Wells, Q. Li, A. R. Moodenbaugh, G. D. Gu, N. Koshizuka, C. Kendziora, S. Jian, and D. G. Hinks. Doping and temperature dependence of the mass enhancement observed in the cuprate $\mathrm{Bi}_{2} \mathrm{Sr}_{2} \mathrm{CaCu}_{2} \mathrm{O}_{8+\delta}$. Phys. Rev . Lett., 87:177007, 2001.

[95] T. Sato, H. Matsui, T. Takahashi, H. Ding, H. B. Yang, S. C. Wang, T. Fujii, T. Watanabe, A. Matsuda, T. Terashima, and K. Kadowaki. 
Observation of band renormalization effects in hole-doped high- $T_{c}$ superconductors. Phys. Rev. Lett., 91:157003, 2003.

[96] T. Cuk, F. Baumberger, D. H. Lu, N. Ingle, X. J. Zhou, H. Eisaki, N. Kaneko, Z. Hussain, T. P. Devereaux, N. Nagaosa, and Z. X. Shen. Coupling of the $B_{1 g}$ phonon to the antinodal electronic states of $\mathrm{Bi}_{2} \mathrm{Sr}_{2} \mathrm{Ca}_{0.92} \mathrm{Y}_{0.08} \mathrm{Cu}_{2} \mathrm{O}_{8+\delta}$. Phys. Rev. Lett., 93:117003, 2004.

[97] T. P. Devereaux, T. Cuk, Z. X. Shen, and N. Nagaosa. Anisotropic electron-phonon interaction in the cuprates. Phys. Rev. Lett., 93:117004, 2004.

[98] X. J. Zhou, T. Yoshida, A. Lanzara, P. V. Bogdanov, S. A. Kellar, K. M. Shen, W. L. Yang, F. Ronning, T. Sasagawa, T. Kakeshita, T. Noda, H. Eisaki, S. Uchida, C. T. Lin, F. Zhou, J. W. Xiong, W. X. Ti, Z. X. Zhao, A. Fujimori, Z. Hussain, and Z. X. Shen. Universal nodal Fermi velocity. Nature, 423:398, 2003.

[99] G. H. Gweon, T. Sasagawa, S. Y. Zhou, J. Graf, H. Takagi, D. H. Lee, and A. Lanzara. An unusual isotope effect in a high-transitiontemperature superconductor. Nature, 430:187-190, 2004.

[100] J. F. Douglas, H. Iwasawa, Z. Sun, A. V. Fedorov, M. Ishikado, T. Saitoh, H. Eisaki, H. Bando, T. Iwase, A. Ino, M. Arita, K. Shimada, H. Namatame, M. Taniguchi, T. Masui, S. Tajima, K. Fujita, S. Uchida, Y. Aiura, and D. S. Dessau. Superconductors: unusual oxygen isotope effects in cuprates? Nature, 446:E5, 2007. 
[101] J. F. Douglas, J. D. Koralek, Z. Sun, N. C. Plumb, Q. Wang, T. J. Reber, J. D. Griffith, Y. Aiura, K. Oka, H. Eisaki, D. S. Dessau, T. P. Devereaux, and S. S. Johnson. N9:6 Laser-ARPES studies of BSCCObased cuprate superconductors. In March Meeting 2007 Program and Show Guide, page 303, 2007.

[102] G. M. Zhao, K. K. Singh, A. P. B. Sinha, and D. E. Morris. Oxygen isotope effect on the effective mass of carriers from magnetic measurements on $\mathrm{La}_{2-x} \mathrm{Sr}_{x} \mathrm{CuO}_{4}$. Phys. Rev. B, 52:6840-6844, 1995.

[103] R. Khasanov, A. Shengelaya, K. Conder, E. Morenzoni, I. M. Savić, J. Karpinski, and H. Keller. Correlation between oxygen isotope effects on transition temperature and magnetic penetration depth in hightemperature superconductors close to optimal doping. Phys. Rev. B, 74:064504, 2006.

[104] G. M. Zhao, K. Conder, H. Keller, and K. A. Müller. Oxygen isotope effects in $\mathrm{La}_{2-x} \mathrm{Sr}_{x} \mathrm{CuO}_{4}$ : evidence for polaronic charge carriers and their condensation. J. Phys. Condens. Matter, 10:9055-9066, 1998.

[105] D. E. Farrell, B. S. Chandrasekhar, M. R. DeGuire, M. M. Fang, V. G. Kogan, J. R. Clem, and D. K. Finnemore. Superconducting properties of aligned crystalline grains of $\mathrm{Y}_{1} \mathrm{Ba}_{2} \mathrm{Cu}_{3} \mathrm{O}_{7-\delta}$. Phys. Rev. B, 36:40254027, 1987.

[106] M. Staines, J. Y. Genoud, A. Mawdsley, and V. Manojlovic. Thick film biaxially textured YBCO coated tape conductors prepared using 
magnetic grain alignment. IEEE Trans. Appl. Supercond., 9:2284-2287, 1999.

[107] A. Trajnerowicz, A. Golnik, C. Bernhard, L. Machtoub, C. Ulrich, J. L. Tallon, and M. Cardona. Isotope effect on the optical phonons of $\mathrm{YBa}_{2} \mathrm{Cu}_{4} \mathrm{O}_{8}$ studied by far-infrared ellipsometry and Raman scattering. Phys. Rev. B, 74:104513, 2006.

[108] E. T. Heyen, R. Liu, C. Thomsen, R. Kremer, and M. Cardona. Optical phonons in $\mathrm{YBa}_{2} \mathrm{Cu}_{4} \mathrm{O}_{8}$ and $\mathrm{Y}_{2} \mathrm{Ba}_{4} \mathrm{Cu}_{7} \mathrm{O}_{15-\delta}$. Phys. Rev. B, 41:1105811067, 1990.

[109] D. Shoenberg. Superconductivity., chapter 5, page 164. Cambridge University Press, Cambridge, England, second edition, 1952.

[110] A. Porch, J. R. Cooper, D. N. Zheng, J. R. Waldram, A. M. Campbell, and P. A. Freeman. Temperature dependent magnetic penetration depth of $\mathrm{Co}$ and $\mathrm{Zn}$ doped $\mathrm{YBa}_{2} \mathrm{Cu}_{3} \mathrm{O}_{7}$ obtained from the $\mathrm{AC}$ susceptibility of magnetically aligned powders. Physica C, 214:350-358, 1993.

[111] A. M. Campbell, F. J. Blunt, J. D. Johnson, and P. A. Freeman. Quantitative determination of percentage superconductor in a new compound. Cryogenics, 31:732-737, 1991.

[112] C. Panagopoulos, J. L. Tallon, and T. Xiang. Effects of the Cu-O chains on the anisotropic penetration depth of $\mathrm{YBa}_{2} \mathrm{Cu}_{4} \mathrm{O}_{8}$. Phys. Rev. B, 59:R6635-R6638, 1999. 
[113] D. N. Basov, R. Liang, D. A. Bonn, W. N. Hardy, B. Dabrowski, M. Quijada, D. B. Tanner, J. P. Rice, D. M. Ginsberg, and T. Timusk. In-plane anisotropy of the penetration depth in $\mathrm{YBa}_{2} \mathrm{Cu}_{3} \mathrm{O}_{7-x}$ and $\mathrm{YBa}_{2} \mathrm{Cu}_{4} \mathrm{O}_{8}$ superconductors. Phys. Rev. Lett., 74:598-601, 1995.

[114] N. Watanabe, S. Adachi, S. Tajima, H. Yamauchi, and N. Koshizuka. Phonon Raman scattering in polycrystalline $\mathrm{Y}_{1-x} \mathrm{Pr}_{x} \mathrm{Ba}_{2} \mathrm{Cu}_{4} \mathrm{O}_{8}$. Phys. Rev. B, 48:4180-4183, 1993.

[115] R. Khasanov, A. Shengelaya, M. Morenzoni, M. Angst, K. Conder, I. M. Savić, D. Lampakis, E. Liarokapis, A. Tatsi, and H. Keller. Siteselective oxygen isotope effect on the magnetic-field panetration depth in underdoped $\mathrm{Y}_{0.6} \mathrm{Pr}_{0.4} \mathrm{Ba}_{2} \mathrm{Cu}_{3} \mathrm{O}_{7-\delta}$. Phys. Rev. B, 68:220506(R), 2003.

[116] L. N. Bulaevskii. Magnetic properties of layered superconductors with weak interaction between the layers. Sov. Phys. JETP, 37:1133-1136, 1973.

[117] V. Ambegaokar and A. Baratoff. Tunneling between superconductors. Phys. Rev. Lett., 10:486-489, 1963.

[118] V. Ambegaokar and A. Baratoff. Tunneling between superconductors (errata). Phys. Rev. Lett., 11:104, 1963.

[119] N. E. Hussey, K. Nozawa, H. Takagi, S. Adachi, and K. Tanabe. Anisotropic resistivity of $\mathrm{YBa}_{2} \mathrm{Cu}_{4} \mathrm{O}_{8}$ : incoherent-to-metallic crossover in the out-of-plane transport. Phys. Rev. B, 56:R11423-R11426, 1997. 
[120] N. E. Hussey, M. Kibune, H. Nakagawa, N. Miura, Y. Iye, H. Takagi, S. Adachi, and K. Tanabe. Magnetic field induced dimensional crossover in the normal state of $\mathrm{YBa}_{2} \mathrm{Cu}_{4} \mathrm{O}_{8}$. Phys. Rev. Lett., 80:29092912, 1998.

[121] G. Rickayzen. The theory of Bardeen, Cooper, and Schrieffer. In R. D. Parks, editor, Superconductivity., volume 1, chapter 2, page 79. Marcel Dekker, New York, 1969.

[122] J. W. Loram, J. Luo, J. R. Cooper, W. Y. Liang, and J. L. Tallon. Evidence on the pseudogap and condensate from the electronic specific heat. J. Phys. Chem. Solids., 62:59-64, 2001.

[123] J. G. Storey, J. L. Tallon, G. V. M. Williams, and J. W. Loram. Fermi arcs in cuprate superconductors: tracking the pseudogap below $T_{c}$ and above T*. Phys. Rev. B, 76:060502(R), 2007.

[124] C. Zhou and H. J. Schulz. Density of states and tunneling spectra in two-dimensional $d$-wave superconductors. Phys. Rev. B, 45:7397-7401, 1992.

[125] S. V. Borisenko, A. A. Kordyuk, T. K. Kim, S. Legner, K. A. Nenkov, M. Knupfer, M. S. Golden, J. Fink, H. Berger, and R. Follath. Superconducting gap in the presence of bilayer splitting in underdoped (Pb,Bi $)_{2} \mathrm{Sr}_{2} \mathrm{CaCu}_{2} \mathrm{O}_{8+\delta}$. Phys. Rev. B, 66:140509(R), 2002.

[126] A. A. Kordyuk, S. V. Borisenko, M. Knupfer, and J Fink. Measuring the gap in angle-resolved photoemission experiments on cuprates. Phys. Rev. B, 67:064504, 2003. 
[127] J. L. Tallon and J. W. Loram. The doping dependence of $T^{*}$ - what is the real high- $T_{c}$ phase diagram? Physica C, 349:53-68, 2001.

[128] P. W. Anderson. The theory of superconductivity in the high-T cuprates. Princeton University Press, 1997.

[129] J. W. Loram, K. A. Mirza, J. R. Cooper, N. Athanassopoulou, and W. Y. Liang. Thermodynamic evidence on the superconducting and normal state energy gaps in $\mathrm{La}_{2-x} \mathrm{Sr}_{x} \mathrm{CuO}_{4}$. In Proceedings of 10th Anniversary HTS Workshop, pages 341-344, Singapore, 1996. World Scientific.

[130] S. H. Naqib, J. R. Cooper, J. L. Tallon, R. S. Islam, and R. A. Chakalov. Doping phase diagram of $\mathrm{Y}_{1-x} \mathrm{Ca}_{x} \mathrm{Ba}_{2}\left(\mathrm{Cu}_{1-y} \mathrm{Zn}_{y}\right)_{3} \mathrm{O}_{7-\delta}$ from transport measurements: tracking the pseudogap below $T_{c}$. Phys. Rev. B, 71:054502, 2005 .

[131] J. Zaanen and B. Hosseinkhani. Thermodynamics and quantum criticality in cuprate superconductors. Phys. Rev. B, 70:060509(R), 2004.

[132] J. C. Campuzano, H. Ding, M. R. Norman, H. M. Fretwell, M. Randeria, A. Kaminski, J. Mesot, T. Takeuchi, T. Sato, T. Yokoya, T. Takahashi, T. Mochiku, K. Kadowaki, P. Guptasarma, D. G. Hinks, Z. Konstantinovic, Z. Z. Li, and H. Raffy. Electronic spectra and their relation to the $(\pi, \pi)$ collective mode in high- $T_{c}$ superconductors. Phys. Rev. Lett., 83:3709-3712, 1999.

[133] N. Miyakawa, P. Guptasarma, J. F. Zasadzinski, D. G. Hinks, and K. E. Gray. Strong dependence of the superconducting gap on oxygen 
doping from tunneling measurements on $\mathrm{Bi}_{2} \mathrm{Sr}_{2} \mathrm{CaCu}_{2} \mathrm{O}_{8-\delta}$. Phys. Rev. Lett., 80:157-160, 1998.

[134] C. Kendziora and A. Rosenberg. $a-b$ plane anisotropy of the superconducting gap in $\mathrm{Bi}_{2} \mathrm{Sr}_{2} \mathrm{CaCu}_{2} \mathrm{O}_{8+\delta}$. Phys. Rev. B, 52:R9867-R9870, 1995.

[135] D. E. Sheehy, T. P. Davis, and M. Franz. Unified theory of $a b$-plane and $c$-axis penetration depths of underdoped cuprates. Phys. Rev. B, 70:054510, 2004 .

[136] R. Micnas and B. Tobijaszewska. Superfluid properties of the extended Hubbard model with intersite electron pairing. J. Phys. Cond. Mat., 14:9631-9649, 2002.

[137] C. Panagopoulos, B. D. Rainford, J. R. Cooper, W. Lo, J. L. Tallon, J. W. Loram, J. Betouras, Y. S. Wang, and C. W. Chu. Effects of carrier concentration on the superfluid density of high- $T_{c}$ cuprates. Phys. Rev. $B, 60: 14617-14620,1999$.

[138] J. L. Tallon, J. W. Loram, J. R. Cooper, C. Panagopoulos, and C. Bernhard. Superfluid density in cuprate high- $T_{c}$ superconductors: A new paradigm. Phys. Rev. B, 68:180501(R), 2003.

[139] W. Anukool. PhD thesis, U. of Cambridge, England, 2003.

[140] F. Wenger and M. Käll. Screened Raman response in two-dimensional $d_{x^{2}-y^{2}}$-wave superconductors: Relative intensities in different symmetry channels. Phys. Rev. B, 55:97-100, 1997. 
[141] J. Thoma, S. Tewari, J. Ruvalds, and C. T. Rieck. Susceptibility and Knight-shift anomalies in cuprate superconductors. Phys. Rev. B, 51:15393-15401, 1995.

[142] K. Asayama, Y. Kitaoka, G. Zheng, and K. Ishida. NMR studies of high $T_{c}$ superconductors. Prog. Nucl. Mag. Res. Spec., 28:221-253, 1996.

[143] A. A. Gippius, E. V. Antipov, W. Hoffman, K. Lüders, and G. Buntkowsky. Low-frequency spin dynamics as probed by ${ }^{63} \mathrm{Cu}$ and ${ }^{199} \mathrm{Hg}$ NMR in $\mathrm{HgBa}_{2} \mathrm{CuO}_{4+\delta}$ superconductors with different oxygen content. Phys. Rev. B, 59:654-660, 1999.

[144] G. V. M. Williams, J. L. Tallon, R. Michalak, and R. Dupree. NMR studies of overdoped $\mathrm{Y}_{1-x} \mathrm{Ca}_{x} \mathrm{Ba}_{2} \mathrm{Cu}_{3} \mathrm{O}_{7-\delta}$. Phys, Rev. B, 57:86968701, 1998.

[145] S. V. Borisenko, A. A. Kordyuk, V. Zabolotnyy, J. Geck, D. Inosov, A. Koitzsch, J. Fink, M. Knupfer, B. Büchner, V. Hinkov, C. T. Lin, B. Keimer, T. Wolf, S. G. Chiuzbăian, L. Patthey, and R. Follath. Kinks, nodal bilayer splitting and interband scattering in $\mathrm{YBa}_{2} \mathrm{Cu}_{3} \mathrm{O}_{6+\delta}$. Phys. Rev. Lett., 96:117004, 2006.

[146] S. D. Obertelli, J. R. Cooper, and J. L. Tallon. Systematics in the thermoelectric power of high- $T_{c}$ oxides. Phys. Rev. B, 46:14928-14931, 1992. 
[147] P. B. Allen, W. E. Pickett, and H. Krakauer. Anisotropic normalstate transport properties predicted and analyzed for high- $T_{c}$ oxide superconductors. Phys. Rev. B, 37:7482-7490, 1998.

[148] G. C. McIntosh and A. B. Kaiser. van Hove scenario and thermopower behavior of the high- $T_{c}$ cuprates. Phys. Rev. B, 54:12569-12575, 1996.

[149] J. Hwang, T. Timusk, and G. D. Gu. High-transition-temperature superconductivity in the absence of the magnetic-resonance mode. $\mathrm{Na}$ ture, 427:714-717, 2004.

[150] A. Kaminski, J. Mesot, H. Fretwell, J. C. Campuzano, M. R. Norman, M. Randeria, H. Ding, T. Sato, T. Takahashi, T. Mochiku, K. Kadowaki, and H. Hoechst. Quasiparticles in the superconducting state of $\mathrm{Bi}_{2} \mathrm{Sr}_{2} \mathrm{CaCu}_{2} \mathrm{O}_{8+\delta}$. Phys. Rev. Lett., 84:1788-1791, 2000.

[151] F. Munakata, K. Matsuura, K. Kubo, T. Kawano, and H. Yamauchi. Thermoelectric power of $\mathrm{Bi}_{2} \mathrm{Sr}_{2} \mathrm{Ca}_{1-x} \mathrm{Y}_{x} \mathrm{Cu}_{2} \mathrm{O}_{8+y}$. Phys. Rev. B, 45:10604-10608, 1992.

[152] D. Mandrus, L. Forro, C. Kendziora, and L. Mihaly. Two-dimensional electron localization in bulk single crystals of $\mathrm{Bi}_{2} \mathrm{Sr}_{2} \mathrm{Y}_{x} \mathrm{Ca}_{1-x} \mathrm{Cu}_{2} \mathrm{O}_{8}$. Phys. Rev. B, 44:2418-2421, 1991.

[153] G. V. M. Williams and J. L. Tallon. Unpublished.

[154] H. J. Trodahl. Thermopower of the superconducting cuprates. Phys. Rev. B, 51:6175-6178, 1995. 
[155] T. Kondo, T. Takeuchi, S. Tsuda, and S. Shin. Electrical resistivity and scattering processes in $(\mathrm{Bi}, \mathrm{Pb})_{2}(\mathrm{Sr}, \mathrm{La})_{2} \mathrm{CuO}_{6+\delta}$ studied by angleresolved photoemission spectroscopy. Phys. Rev. B, 74:224511, 2006.

[156] T. Valla, A. V. Fedorov, P. D. Johnson, Q. Li, G. D. Gu, and N. Koshizuka. Temperature dependent scattering rates at the Fermi surface of optimally doped $\mathrm{Bi}_{2} \mathrm{Sr}_{2} \mathrm{CaCu}_{2} \mathrm{O}_{8+\delta}$. Phys. Rev. Lett., 85:828$831,2000$.

[157] Y. Wang, J. Yan, L. Shan, H. H. Wen, Y. Tanabe, T. Adachi, and Y. Koike. Weak coupling $d$-wave BCS superconductivity and unpaired electrons in overdoped $\mathrm{La}_{2-x} \mathrm{Sr}_{x} \mathrm{CuO}_{4}$ single crystals. Phys. Rev. B, 76:064512, 2007.

[158] S. Sugai and T. Hosokawa. Relation between the superconducting gap energy and the two-magnon Raman peak energy in $\mathrm{Bi}_{2} \mathrm{Sr}_{2} \mathrm{Ca}_{1-x} \mathrm{Y}_{x} \mathrm{Cu}_{2} \mathrm{O}_{8+\delta}$. Phys. Rev. Lett., 85:1112-1115, 2000.

[159] A. W. Hunt, P. M. Singer, K. R. Thurber, and T. Imai. ${ }^{63} \mathrm{Cu}$ NQR measurement of stripe order parameter in $\mathrm{La}_{2-x} \mathrm{Sr}_{x} \mathrm{CuO}_{4}$. Phys. Rev. Lett., 82:4300-4303, 1999.

[160] P. M. Singer and T. Imai. Systematic ${ }^{63} \mathrm{Cu}$ NQR and ${ }^{89} \mathrm{Y}$ NMR study of spin dynamics in $\mathrm{Y}_{1-z} \mathrm{Ca}_{z} \mathrm{Ba}_{2} \mathrm{Cu}_{3} \mathrm{O}_{y}$ across the superconductor insulator boundary. Phys. Rev. Lett., 88:187601, 2002.

[161] Y. Kohsaka, C. Taylor, K. Fujita, A. Schmidt, C. Lupien, T. Hanaguri, M. Azuma, M. Takano, H. Eisaki, H. Takagi, S. Uchida, and J. C. 
Davis. An intrinsic bond-centered electronic glass with unidirectional domains in underdoped cuprates. Science, 315:1380-1385, 2007.

[162] N. Nishida, S. Okuma, H. Miyatake, T. Tamegai, Y. Iye, R. Yoshizaki, K. Nishiyama, K. Nagamine, R. Kadono, and J. H. Brewer. Internal magnetic fields at $\mu^{+}$sites and the magnetic phase diagram of $\mathrm{Bi}_{2} \mathrm{Sr}_{2} \mathrm{Y}_{1-x} \mathrm{Ca}_{x} \mathrm{Cu}_{2} \mathrm{O}_{y}$ system studied by the $\mu$ SR method. Physica $C$, 168:23-28, 1990.

[163] M. Platé, J. D. F. Mottershead, I. S. Elfimov, D. C. Peets, R. Liang, D. A. Bonn, W. N. Hardy, S. Chiuzbaian, M. Falub, M. Shi, L. Patthey, and A. Damascelli. Fermi surface and quasiparticle excitations of overdoped $\mathrm{Tl}_{2} \mathrm{Ba}_{2} \mathrm{CuO}_{6+\delta}$. Phys. Rev. Lett., 95:077001, 2005.

[164] C. C. Torardi, M. A. Subramanian, J. C. Calabrese, J. Gopalakrishnan, E. M. McCarron, K. J. Morrissey, T. R. Askew, R. B. Flippen, U. Chowdhry, and A. W. Sleight. Structures of the superconducting oxides $\mathrm{Tl}_{2} \mathrm{Ba}_{2} \mathrm{CuO}_{6}$ and $\mathrm{Bi}_{2} \mathrm{Sr}_{2} \mathrm{CuO}_{6}$. Phys. Rev. B, 38:225-231, 1988.

[165] H. Eisaki, N. Kaneko, D. L. Feng, A. Damascelli, P. K. Mang, K. M. Shen, Z. X. Shen, and M. Greven. Effect of chemical inhomogeneity in bismuth-based copper oxide superconductors. Phys. Rev. B, 69:064512, 2004 .

[166] H. J. Bornemann, D. E. Morris, H. B. Liu, and P. K. Narwankar. Dependence of the oxygen isotope effect upon hole density in $\mathrm{Bi}_{2} \mathrm{Sr}_{2} \mathrm{Ca}_{1-x} \mathrm{Y}_{x} \mathrm{Cu}_{2} \mathrm{O}_{8+\delta}$ and $\mathrm{Y}_{1-x} \mathrm{Ca}_{x} \mathrm{Ba}_{2} \mathrm{Cu}_{4} \mathrm{O}_{8}$. Physica C, 191:211218, 1992. 
[167] N. Suresh, J. G. Storey, G. V. M. Williams, and J. L. Tallon. Strong pressure dependence of the oxygen isotope effect in $\mathrm{YBa}_{2} \mathrm{Cu}_{4} \mathrm{O}_{8}$. unpublished.

[168] N. Doiron-Leyraud, C. Proust, D. LeBoeuf, J. Levallois, J-B. Bonnemaison, R. Liang, D. A. Bonn, W. N. Hardy, and L. Taillefer. Quantum oscillations and the Fermi surface in an underdoped high- $T_{c}$ superconductor. Nature, 447:565-568, 2007.

[169] E. A. Yelland, J. Singleton, C. H. Mielke, N. Harrison, F. F. Balakirev, B. Dabrowski, and J. R. Cooper. Quantum oscillations in the underdoped cuprate $\mathrm{YBa}_{2} \mathrm{Cu}_{4} \mathrm{O}_{8}$. arXiv:0707.005\%, 2007.

[170] O. Chmaissem, J. D. Jorgensen, S. Short, A. Knizhnik, Y. Eckstein, and $\mathrm{H}$. Shaked. Scaling of transition temperature and $\mathrm{CuO}_{2}$ plane buckling in a high-temperature superconductor. Nature, 397:45-48, 1999.

[171] D. M. Newns and C. C. Tsuei. Fluctuating Cu-O-Cu bond model of high-temperature superconductivity. Nature Physics, 3:184-191, 2007. 